$\mathrm{DOE} / \mathrm{BC} / 14251--9$

Distribution DE92 001029

\title{
RESERVOIR CHARACTERIZATION AND ENHANCED OIL RECOVERY RESEARCH
}

Annual Report for the Period

September 1988 to August 1989

\author{
By \\ Larry W. Lake \\ Gary A. Pope \\ Robert S. Schechter
}

March 1992

Work Performed Under Contract No. DE-FG22-89BC14251

Prepared for

U.S. Department of Energy

Assistant Se cretary for Fossil Energy

Jerry Ham. Project Manager

Metairie Site Office

900 Commerce Road. East

New Orleans. LA 70123

\author{
Prepared by \\ University of Texas \\ Center for Petroleum and Geosystems Engineering \\ Ausin, TX 78712
}




\section{ABSTRACT}

The research in this annual report falls into three tasks each dealing with a different aspect of enhancéd oil recovery.

The first task strives to develop procedures for accurately modeling reservoirs for use as input to numerical simulation flow models. This section describes how we have used a detailed characterization of an outcrop to provide insights into what features are important to fluid flow modeling. The work leads to a discussion of pseudo functions and how they are used in reservoir modeling to capture geologic features, and also to the notion of a test case for measuring the utility of statistical models. The work describes diagnetic patterns which occur in a carbonate outcrop and attempts to explain these from a fluid flowreaction model.

The second task deals with scaling-up and modeling chemical and solvent EOR processes. In a sense this task is the natural extension of task 1 and, in fact, one of the subtasks uses many of the same statisticai procedures for insight into the effects of viscous fingering and heterogeneity. Other subtasks use UTCHEM, a compositional chemical flooding numerical model, to estimate reservoir properties from single-well tests, and apply expert system approaches to design chemical and compositional model input. A largely experimental subtask investigates the effects of cation exchange capacity and chromium retention of the polymer gel process.

The final task involves surfactants and their interactions with carbon dioxide and reservoir minerals. This research deals primarily with phenomena observed when aqueous surfactant solutions are injected into oil reservoirs. The reason for injecting surfactant solutions is to render the displacement of oil by carbon dioxide a more efficient process. Aqueous surfactant solutions may disrupt the stability of thin aqueous films, characteristic of water-wet rocks, surfactant solutions may adsorb onto the rock, or surfactant solutions may interact with oil to produce microemulsions. All of these effects are detrimental to the efficiency of carbon dioxide mobility control by foams and all are discussed in the final section of this report. 


\section{EXECUTIVE SUMMARY}

The first part of this annual report discusses the research concentrating on atterapts to develop a systematic procedure for reservoir characterization.

Subtask 1a, "Development of an Averaging Procedure for Relative Permeability which Accounts for Both Continuity and Cross-bedding," is largely complete. We developed a procedure for deriving effective or pseudo relative permeabilities for viscouslydominated flow in cross-bedded sands. The procedure makes use of the observation that cross-bedding, as represented through a tensorial permeability, can be accurately represented by isotropic permeabilities which have been corrected for the cross-bedding. This observation, which was extensively validated with detailed simulation, means that the Hearn technique can be applied to flow in cross-bedded media without significant error. Although the method was derived specifically for flow through eolian sequences, the procedure should be valid for any cross-bedded sand.

We applied the effective relative permeabilicies to a viscously-dominated, highly detailed simulation of a miscible displacement through a portion of the Page Sandstone outcrop. Using the effective properties resulted in a reduction in the number of finite element nodes from more than 11,000 to 36 with essentially the same recovery function. Since this displacements was miscible, the effective relative permeabilities incorporate the details of the underlying geology.

For Subtask 1b, "Quantification of Existing Taxonomy Through the Use of Correlation, Fractal Representation and Nonlinear Dynamic Statistical Procedures," we have taken extensive data on the distribution of permeability in the San Andres formation from the North Lawyer Canyon region of the Algerita outcrop in southern New Mexico. We find good agreement between these data and similar data taken from wells in the nearby Wasson field.

Permeability showed substantial variability within individual bedded units arranged in three distinct scales of correlation. Outcrop permeability data exhibited no marked permeability anisotropy in predicted spatial correlation length. Several scales of spatial variability have been observed in an outcrop section, with subsurface results in agreement.

We will report fully on Subtask 1c, "Use of Geochemical Flow Modeling to Determine the Geometric Patterns in Porosity and Pormeability that Result from Diagenetic Processes," in the final report. The avenue of attack for this problem is to initiate a fundamental study of reactive flow in the presence of a combination of reactions both in 
and out of local thermodynamic equilibrium. To proceed along the latter, we have solved a reactive problem for flow through a tube with the intent of using these insights to broaden the work to flow through permeable media. The results of the tube model are to be used in a network simulation to identify circumstances when dissolution or precipitation (calcification) would occur.

For Subtask 1d, "Development of an Experimental Validation Procedure Using Simulated Fluid-Flow to Quantify the Amount of Geologic Detail Required for a Satisfactory Characterization," we used a finite element numerical model to simulate flow through an outcrop of the eolian Page Sandstone. The results of the simulated miscible displacements show that the relative positions of the main geologic features and the ratio between the grainflow and windripple permeabilities are more important than bounding surfaces and dispersion in determining flow behavior. In the presence of the large-scale heterogeneities, uncorrelated small-scale heterogeneities do not affect the sweep efficiency and the recovery.

Subtask 2 addresses the overall goal of modeling and scaleup of chemical flooding with a focus on polymer gels for profile modification and chemical tracers. Subtask $2 \mathrm{a}$ of our report presents "Single-Well Tracer Studies to Estimate Reservoir Properties Other Than Residual Oil Saturation." It focuses on a two-dimensional radial coordinate option added to The University of Texas Chemical Flooding Simulator (UTCHEM). The mathematical formulation of the radial option and its implementation in UTCHEM has been completed. Comparisons of simulation results to an analytical solution of the radial convection-dispersion equation show that the simulator accurately models dispersive radial flow to the point that numerical dispersion control may not be the limiting factor in grid block size.

Subtask $2 b$ is on the "In-Situ Behavior of Gels Formed While Flowing in Permeable Media." The competition for chromium between xanthan biopolymer and the resident ciays in Berea sandstone cores has been examined. Through a series of Berea corefloods, chromium retention was examined without the presence of polymer using a 2 $w_{t} \% \mathrm{NaCl}$ brine as the aqueous solution. The retention of chromium was found to be high and on the order of the cation exchange capacity of the clays in the sandstone.

Subtask $2 c$ reports on the "Simulation of Hydrodynamic Instabilities (Viscous Fingering) in Permeable Media." Fine-mesh simulations of first-contact miscible displacements have been performed using UTCHEM. The results match the production history of a laboratory-scale coreflood. An empirical viscous fingering model was 
implemented and tested. The model can successfully match the recovery curve of a firstcontact miscible linear unstable displacement.

Subtask 2d of this report is on the "Development of an 'Expert System' to Design Chemical Floods Using a Compositional Reservoir Simulaıor." In order to develop a rulebased computer program, the user directs the program to design chemical floods by picking items from descriptive menus offered by the program. There is extensive use of computer graphics to show the user the effects of varying input values before running the simulation and to plot the results of the simulation so they may be easily interpreted. The program is implemented for use with UTCHEM, but the technique used to write the program is general enough to allow it to be applied to other simulators with little effort.

The tendency for less viscous carbon dioxide $\left(\mathrm{CO}_{2}\right)$ to by-pass large volumes of more viscous crude oil substantially diminishes the economic viability of enhanced oil recovery processes which use $\mathrm{CO}_{2}$ as the displacing agent. Foams formed in-situ represent a possible means for controlling the mobility of $\mathrm{CO}_{2}$ but the process is complex. There are many phenomena that take place whenever aqueous surfactant solutions are injected into oil-bearing formations and some of these are detrimental to the process. One crucial issue is the need to maintain or restore water-wettability so that foam can form. Indeed, two of the research projects reported in Task 3 relate to this problem. One is concerried with the stability of thin wetting films and a technique for establishing the thickness of a water film on a quartz surface as a function of the disjoining pressure has been established. Results that agree with previously reported values have been obtained. However, the variation of the thickness of a thin liquid film resting on a quartz plate forming the top of a small cylindrical cavity has produced some rather surprising results; namely, regions predicted to be essentially flat based on the modified Laplace equation exhibit a finite, measurable curvature thus bringing into question the validity of this fundamental equation which is widely used for characterizing the wettability of a surface. Work on this problem is continuing.

Subtask 3a reports on Microemulsion and $\mathrm{CO}_{2}$-Surfactants-Water Interactions. Aqueous surfactant solutions can interact with the oil present in the formation to produce stable microemulsions. To develop some understanding as to the rate that this can occur, nonequilibrium diffusion in microemulsions has been studied using the open capillary method. Gradients in concentration of each component as well as solution microstructure were present in these experiments. The open capillary experiment is shown to be useful for studying the diffusion behavior of microemulsions. The existing theory was found to 
predict diffusion in Winsor I microemulsions; however, in the single-phase regions near the point where three-phase systems tend to form (Winsor III systems), there are sharp differences between the predicted and the obser ad behavior. An adequate theory does not exist to describe such systems.

The stability of thin aqueous films represents one aspect of the problem, but other important questions relate to the rate at which lamella form at pore throats and how this rate depends on the formation wettability, formation pore structure, and flow rates. We have not yet formulated our answer to these questions, but progress has been made. Subtask $3 \mathrm{~b}$, an analysis of the stability of thin films and liquid collars in pore throats is presented here. The main results found this year relate to the theoretical method used in the determination of stability and to the implementation of this method. Future work will stress the practical implications of this theoretical result.

To create stable ioams, surfactants must be introduced into the formation but these surfactants can adsorb onto the mineral surfaces and be lost. Furthermore, the adsorption at low surfactant concentrations is the important feature of the isotherm, not the absorption at high concentrations. Studies under Subtask 3c, "Wettability and Adsorption," have resulted in the development of a new model applicable to low surfactant concentrations. It is intended to be comprehensive. Included are the effects of $\mathrm{pH}$ and electrolyte composition as well as essential parameters characterizing the nature of the surface. This new model also includes surfactant structure as a parameter and introduces a new mode of adsorption - partial monolayer clustering - which has not heretofore been reported. Unless this mode of adsorption is taken into account, model predictions for adsorption will be in error. Generally, when neglecting this new mode of adsorption, the level of adsorption predicted will underestimate that actually observed. 


\title{
TASK 1 - SYSTEMATIC PROCEDURE FOR RESERVOIR CHARACTERIZATION
}

\author{
Subtask 1a - Development of an Averaging Procedure for Relative \\ Permeability which Accounts for both "Continuity" and "Cross-bedding."
}

We developed a procedure for deriving effective or pseudo relative permeabilities for viscously-dominated flow in cross-bedded sands. The procedure makes use of the observation that cross-bedding, as represented through a tensorial permeability, can be accurately represented by isotropic permeabilities which have been corrected for the crossbedding. This observation, which was extensively validated with detailed simulation, means that the Hearn technique can be applied to this geologically complicated flow without significant error. Although the method was derived specifically for eolian sequences, the procedure should be valid for any cross-bedded sand.

We applied the effective relative permeabilities to a viscously-dominated, highly detailed simulation of a miscible displacement through a portion of the Page Sandstone outcrop. Using the effective properties results in a reduction in the number of finite element nodes from more than 11,000 to 36 with essentially the same recovery function. Since this displacements was miscible, the effective relative permeabilities incorporate the details of the underlying geology almost exclusively.

\section{Introduction}

Although the development of more powerful computers and simulation techniques allows the use of very fine grids, the size of permeability variations in permeable medium are still smaller than the smallest grids. This situation requires calculating effective or pseudo properties for cells of simulations that possess spatial heterogeneities (Kasap et al. 1990). Since a single effective permeability cannot account for sweep efficiency, one alternative is to use effective relative permeability functions to relate fluid flux to an imposed potential difference on different scales.

There are several difficulties involved in representing the flow properties with effective properties (Laike et al. 1989). Effective properties are usually scale- or timedependent or sometimes both. Furthermore, it is generally not easily possible to validate the calculated results obtained by using the effective properties. 
However, effective properties can actually contain significant advantages because it is through them that we can insert properly-weighted and scale-adjusted transport properties into reservoir calculations. They lump a great deal of fluid and geologic information in a form that can be easily used. Effective properties can be useful in lowering data preparation and computational costs by (1) reducing the number of grid cells in numerical simulations, (2) reducing the spatial dimensions of a problem, (3) accounting for coning, and (4) improving well models in areal simulations.

The effective properties can be in the form of: (1) an effective permeability tensor of a heterogeneous flow unit, (2) effective relative permeabilities (for miscible or immiscible displacenents), (3) an effective dispersion tensor for heterogeneous flow units, (4) an effective capillary pressure curve, and (5) a coning function or a well model for water or gas-drive mechanism.

\section{Literature Survey}

Effective properties are generated in two ways: through averaging the results of a simulation run (numerical methods) with a fine grid and through partial integration of the flow equation (analytical methods), usually in a direction perpendicular to the main flow direction.

Analytical Studies - Nearly all analytical effective relative permeabilities require the assumption of vertical equilibrium (VE). This assumption makes it possible to partially integrate the conservation equations for use in a coarser simulation model or a model of lower dimensionality.

The basic work here is that of Coats et al. (1967 and 1971) where the concept of vertical equilibrium was introduced and applied. The work by Martin (1968) served to put Coats' work on a firmer theoretical foundation and to illustrate certain limiting cases. Limiting cases form the basis of much of the literature, including the viscous-dominated cases described by Hearn (1971), Zapata and Lake (1981), and Hewett and Behrens (1989); they also are the basis of the work in this section. The work of Hawthorne (1975) is a limiting case omitting gravity forces. Extensions of the original Coats theory include the pseudo functions of Killough and Foster (1979) to model the drainage of the Empire Abo field, and the extension by Rosenzweig et al. 1986) to three-phase flow.

Numerical studies - Numerical generation of effective properties is usually more general than assuming VE and has enjoyed considerable popularity in recent years. Usually a cross-section or a flow unit is discretized in very fine detail and the effects of the flow property for which the effective function is being generated is captured. Effective 
properties generated from flow simulations can be dynamic in the sense that they depend on the flow history of the displacement. Dynamic effective properties are generated for simulation cells to account for small-scale heterogeneity (the use here) or gas/water coning.

Typical numerically-generated effective properties iiclude the works of van Pollen et al. (1968), Jacks et al. (1973), and Kyte and Berry (1975) all of which discuss large changes in flow rate or reduced numerical dispersion. Effective properties to account for small-scale geologic features such as discontinuous shales (Davies et al., 1987), laminations (Kortekaas et al., 1983) or features introduced by statistical procedures (Muggeridge, 1989) are also present. Other numerical procedures are intended to account ior differences in the size of grid cells (Starley, 1986) and to detail procedures for scaling up from a local scale (Kossack et al., 1989, Lasseter et al., 1986). Thomas (1983) has attempted to reconcile these various approaches as well as the analytical approaches.

Numerical effective properties cannot easily incorporate scale dependency because they are generated for a specific run (a specific scale, mobility ratio, flow rate, etc.), and are usually time dependent. For practical use effective properties should be independent of time but they may depend on scale and possibly position (Lake et al., 1989).

\section{Objectives}

The objective of this study is to develop an analytical method to describe the effects of cross-bedded units modeled by a simulation cell. We also show that the effective relative permeabilities, applied to the coarse grid simulations, reproduce the results of fine grid simulations reasonably well. See subtask $1 \mathrm{~d}$ for a description of the fine grid results. To accomplish this, we first modeled a cross-bedded flow unit, then developed an analytic method to calculate effecti re relative permeabilities. The modeling procedure consists of three steps: (1) finding a prototype model for the heterngeneity, (2) discretizing the model into a set of uniformly cross-bedded layers, and (3) homogenizing the layers.

The analytic method extends the calculation of effective properties in several aspects. The method is analytic, but it incorporates the advantages of the numerical methods by calculating dynamic effective properties, taking into account the sweep history of a simulation cell. The method can handle tensorial permeabilities and accounts for scaledependency when the simulation cell is only a part of the flow unit. The method is, however, limited to viscously-dominated flows (Hearn, 1971). 


\section{Modeling and Homogenization}

Cross-bedded flow units occur commonly in sandstone reservoirs. In this section, a prototype model was considered for the Page Sandstone (Chandler, 1986, Muskat, 1946). Although the results are specific to the Page Sandstone, the method is valid for all cross-bedded permeable media.

\section{Prototype model}

Figure 1 is a photograph of a portion of the south wall of the Page Sandstone outcrop. The horizontal boundaries of the units D2 and D4 are easily distinguishable in the picture. The thicknesses of both units are 10-12 ft (3-4 m). The cross-bedding is very typical of eolian deposits. Although the shape of these features vary from one unit to another, characteristic features within a unit are repeated in both the horizontal and vertical directions. In general, a characteristic feature a prototype model of a flow unit consists of a combination of windripple and grainflow lamination. The windripple laminations are very thin at the top, widen toward the bottom and become parallel to the bed. The grainflow laminations are large at the top, but diminish toward the bottom.

To characterize a flow unit we developed a prototype model that will represent the heterogeneity within the unit. Figure 2 a shows such a prototype model for the D4 unit. The dark regions represent windripples, with a small permeability, and the light regions represented grainflows with a large permeability. The laminated portion at the top is about $70 \%$ of the unit. The angle of the laminations (measured counter-clockwise from the vertical) increases from top to bottom of the unit.

\section{Discretization}

The next step is to discretize the prototype model into parallel layers. Eight variable-thickness parallel layers are generated from $\mathrm{D} 4$ by discretizing the prototype model. One criterion in generating the layers was to minimize the number of layers while still retaining much of the detail in the cross-bedding. The thickness and the angle of the cross-bedding change within a layer but we treat them as constant at the average of the angles at the top and the bottom of each layer (Fig. 2b). The parameters of the generated layers : $:$ a listed in Table 1. The thicknesses of the cross-beds within a layer are the percentage of the thickness of the small-permeability streaks to the total layer thickness. The set of layers shown in Fig. $2 b$ are a set of uniformly cross-bedded parallel layers. 


\section{Homogenization}

Homogenization of a uniformly cross-bedded unit is a procedure where the layer is substituted by a homogeneous one with a tensorial permeability. Cross-bedding and nonzero off-diagonal elements of the permeability tensor have similar effects on a displacement front. Cross-bedding causes the local streamline direction to be different from the direction of the applied potential gradient; non-zero off-diagonal elemen:s in the permeability tensor have the same effect.

In general, streamlines will not be normal to isopotential lines if permeability is anisotropic (Muskat, 1946; Bear, 1977; Lake, 1988). Scheidegger (1972) suggested that there are three orthogonal axes (the principal axes) in space along which the potential gradient and velocity have the same direction. Greenkorn et al. (1964) considered the permeability tensor to be made up of two components that originate from: (1) anisotropy (variation around a point) which correlates with bedding and (2) heterogeneity (variation from point-to-point) which correlates with grain size variation. The heterogeneity effect is more significant than anisotropy caused by orientation of grains (Greenkorn et al.,1964; Young, 1989).

Procedure - The first step of the homogenization procedure is to calculate the permeabilities in the principal directions of the permeability. The principal directions of the permeability tensor are the directions normal and parallel to the cross-bedding direction. This is true if the permeabilities within the stratification types are isotropic.

Figure 3 shows a cross-bedded medium with alternating permeability streaks. The figure also shows the prameters $\left(\mathrm{k}_{1}, \mathrm{k}_{2}, \mathrm{~h}_{\mathrm{cbl}}, \mathrm{h}_{\mathrm{cb} 2}, \alpha_{\mathrm{cb}}, \mathrm{L}, \mathrm{H}\right.$ and principal directions of permeability, $n$ and $p$ ) used throughout this section to describe a cross-bedded unit. The permeabilities in the principal directions are calculated as functions of the cross-bed thicknesses of large and small permeability streaks and the local permeabilities. The permeability in the normal direction $\left(k_{n}\right)$ is calculated by the harmonic and in the parallel direction $\left(k_{p}\right)$ by the arithmetic averaging.

$$
k_{n}=\frac{\left(h_{c b 1}+h_{c b 2}\right)}{\frac{h_{c b 1}}{k_{1}}+\frac{h_{c b 2}}{k_{2}}} \text { and } \quad k_{p}=\frac{h_{c b 1} k_{1}+h_{c b 2} k_{2}}{h_{c b 1}+h_{c b 2}}
$$

These equations are easily generalized to more than two stratification types.

The second step is to apply a coordinate rotation to arrive at the simulation coordinates (Kasap et ai. 1990). The resulting permeability tensor is a full tensor with non- 
zero off-diagonal terms. From those tensorial permeabilities a single-phase effective permeability for each layer in the $x$-direction can be calculated as the coefficient of the potential difference in the $x$-direction.

$$
\mathrm{u}_{\mathrm{x}}=\frac{1}{\mu} \tilde{\mathrm{k}}_{\mathrm{xi}} \frac{\Delta_{\mathrm{x}} \Phi}{\Delta \mathrm{x}}
$$

The effective permeability is defined as

$$
\tilde{k}_{x i}=k_{x x i}-\frac{k_{x y i}^{2}}{k_{y y i}}, \quad i=1, N_{L}
$$

where $N_{L}$ is the total number of layers. The effective permeabilities are obtained by assuming that the y-component of the velocity vector is zero. We show below that this assumption is valid even in the presence of cross-bedding.

Figure 4 shows the calculated effective permeabilities for each layer in the model of Fig. 2b. The mean permeabilities were 3 and $0.75 \mu \mathrm{m}^{2}$ for grainflov' and windripple structures respectively (Kasap, 1990). All analytical calculations of effective relative permeabilities and recoveries in this study are based on these effective permeabilities.

Validation - Figure 5a shows a cross-section of a uniformly cross-bedded permeable medium. It is uniformly cross-bedded because the thickness and the acute angle of the cross-bedding do not change throughout the cross-section. In Fig. 5a dark and light streaks represent regions with small and large permeability, respectively. The ratio of the large to small permeability is 10 and the thickness of the large permeability streak equal to that of the small permeability streak. The permeabilities are locally isotompic (within the streaks). Tine cross-bedding angle $\alpha_{c b}$ is $\left(\frac{\pi}{4}\right)$ and the aspect ratio, the ratio of the length to the thickness, is 5. Figure 5a also shows the finite element grid scheme (we cannot see the grid on the dark streaks) generated for the numerical simulation of single-phase flow in this cross-bedded section. In the generated grid scheme, triangular elements were used, so that the cruss-bedding can be included explicitly. For the details of the simulator see Kas.ip (1990).

The cross-bedded medium in Fig. 5a is homogenized by replacing it with a homogeneous medium with an anisotropic permeability tensor. Since the principal coordinates of permeability (the direction of cross-bedding and the direction perpendicular 
to the cross-bedding) are not aligned with the Cartesian coordinates, the permeability of the homogenized medium is in a tensorial form with non-zero off-diagonal terms.

To see whether the homogenized medium shows the same fluid flow characteristics as the cross-bedded one, we simulated a matched density and viscosity, dispersion-free miscible displacement in both the cross-bedded and homogenized media. The displacement was therefore controlled exclusively by viscous forces. The same boundary conditions (no-flow along the horizontal boundaries and uniform flux along the vertical boundaries) were used for both media.

Figure 5b shows the potential contours obcained from the simulation in the crossbedded medium in Fig. 5a. The potential contours are fairly straight and uniform except for some minor bending ai the grainflow-windripple boundaries. Figure $5 \mathrm{c}$ shews the potential contours obtained from a simulation in the homogenized version of the crossbedded medium. The acute angle the isopotential lines form with the vertical lines indicates the effect of the tensorial permeability.

The front location of a miscible displacement in a cross-bedded medium may also be compared with one in a homogenized medium. Figure $5 \mathrm{~d}$ shows displacement fronts at 0.4 and 0.7 pore volumes injected (PVI). PVI is the cumulative injected volume given as a fraction of the pore volume. The vertical front represents a displacement in the homogenized cross-section in which potential contours were shown in Fig. 5c. The wiggly front represents a displacement in the cross-bedded system in which the potential contours were shown in Fig. 5b. The agreement between front shapes and especially the locations indicates that the homogenization procedure is valid not only for the potential distribution but also for the displacement, as long as the flow unit contains more than 10 cross-beds Kasap, 1990).

Although Fig. 5 shows that the homogenizing procedure applied to a single crossbedded layer is accurate, it is not yet clear whether this is a general characteristic of a crossbedded mediz or just limited to single layers. To see whether cross-bedding causes crossflow in multi-layered systems, we also studied a medium consisting of three crossbedded layers having common horizontal boundaries. Three cross-bedded layers will show all the characteristics of the multi-l, yered systems, since there exists at least one layer with all boundaries open to flow.

The medium under study consists of three layers, the middle one having the smallest permeability, and all consisting of a different cross-bedding angle. The large and the small permeabilities were 5 times smaller than their counterparts in the top and the bottom layers. All the parameters were the same for the top and the bottorn layers except 
the cross-bedding angle. The aspect ratio was 5 and the thicknesses of the large- and small-permeability streaks were the same for all three layers. The crosi-bedding angles, from top to bottom, were $\pi / 6, \pi / 3$ and $5 \pi / 4$. The ratio between the large and the small permeabilities was 10 .

Figure 6a also shows the fini. element grid generated to simulate fluid flow in this cross-section. Figure $6 \mathrm{~b}$ shows the numerically calculated potential contours in Fig. $6 \mathrm{a}$ which also reflect the cross-bedding orientations within each lay $r$. Figure $6 c$ shows the isopotential lines in the homogenized medium. Figure $6 \mathrm{~d}$ shows analytically calculated front locations for the homogenized cross-section and the numerically calculated front locations for the cross-bedded section. Again the front locations of the two cases compare very well.

From the results presented above we conclude that in a multi-layered medium, flowing a single incompressible fluid, there will be no fluid redistribution (crossflow) within the syctem solely because of cross-bedding. The fluid is distributed among the layers according to their effective permeabilities immediately after entering the system. Comparing Fig. $6 \mathrm{~b}$ with Fig. $6 \mathrm{c}$, we also conclude that the homogenization procedure is valid for multiple parallel layers.

\section{Vertical sween and effective relative nermeability}

Two approaches are often used to calculate vertical sweep efficiency analytically in a cross-section of parallel layers. The first approach assumes no vertical communication between the layers, a condition that would apply in actual practice if the reservoir contained impermeable and continuous shales within the total interval. The second approach assumes VE across the cross-section of the parallel layers.

The assumption of non-communicating layers is not appropriate here, because there are no impermeable and continuous shale breaks in the total interval. Therefore, we calculate vertical sweep efficiency and effective relative permeabilities by assuming VE. The vertical sweep efficiency and effective relative permeabilities are all calculated for single-phase flow where viscous forces are the only driving forces. Even though the procedure is valid for any mobility ratio (the ratio of the viscosity of the displaced fluid to that of the displacing fluid), the example calculations are carried out only for mobility ratios greater than one. 


\section{Vertical equilibrium}

Vertical equilibrium is a condition where the sum of all lateral (vertical) driving forces is zero in the direction normal to the bulk fluid flow (Lake, 1989). If VE holds in segregated flow in a group of parallel layers, there exist reservoir segments in which the potentrai gradient is constant in the horizontal direction. Figure 7 is a schematic illustration of a cross-section in which a more mobile fluid is displacing a less mobile one, withicut mixing (Zapata et al., 1989), and under the state of VE. Figure 7a shows the front positions and the segment boundaries before breakthrough and $7 \mathrm{~b}$ shows the potential distribution in the horizontal direction. In Fig. 7 , $\mathrm{i}$ and $\mathrm{j}$ refer to the layer numbers and the segment numioers, respectively.

Assuming a constant injection rate we can calculate the velocity of the fluid flowing in any segment of any layer. The $x$-direction velocity of the fluid in any segment of a layer can be calculated from the Darcy equation by considering a constant potential gradient in the vertical direction in that segment.

$$
u_{x i j}=\frac{\tilde{k}_{x i}}{\mu_{i j}}\left(\frac{\Delta_{x} \Phi}{\Delta x}\right)_{j}
$$

where $\widetilde{\mathrm{k}}_{\mathrm{xi}}$ is given by Eq. (1), $\mu_{\mathrm{ij}}$ is the viscosity of the fluid in the $\mathrm{j}^{\text {th }}$ segment of the $\mathrm{i}^{\text {th }}$ layer and $\left(\frac{\Delta_{x} \Phi}{\Delta x}\right)_{j}$ is the potential gradient in $j^{\text {th }}$ segment. Since the sum of the fluxes times the layer thickness in any segment is equal to the total volumetric rate.

$$
Q \quad=\left(\frac{\Delta_{x} \Phi}{\Delta x}\right)_{j} \sum_{i=1}^{N_{L}} \frac{\tilde{k}_{x i} h_{i}}{\mu_{i j}}
$$

or

$$
\left(\frac{\Delta_{x} \Phi}{\Delta x}\right)_{j}=\frac{Q}{\sum_{i=1}^{N_{L}} \frac{\tilde{k}_{x i} h_{i}}{\mu_{i j}}}
$$

for a linear medium of unit width. 
Substituting Eq. (3) into (2) gives the $x$-velocity in segment $i$ of layer $j$.

$$
u_{x i j}=\frac{\tilde{k}_{x i}}{\mu_{i j}} \frac{Q}{\sum_{i=1}^{N_{L}} \frac{\tilde{k}_{x i} h_{i}}{\mu_{i j}}}
$$

Equation (4) indicates that the flux in each segment of a specific layer is not a function of location or time (unless $Q$ changes). The flux in each segment, then, will remain the same throughout the displacement process.

The velocities given by Eq. 4 are the velocities on either side (behind and ahead) of the front in a layer. There are basically two approaches to calculate the front velocities. The first approach assumes the displacing and displaced fluids are segregated (no mixing); it solves the material balance equations derived for either side of the front in a layer. The segregated flow assumption is too restrictive, cause very often there will be mixing around the front which causes the faster layer-front to slow down and slower layer-front to speed up; therefore, neglecting the mixing in front velocity calculations leads to a recovery prediction that is too pessimistic. The second approach is to account for the mixing around the front. Zapata et al. developed a rigorous analytic technique to account for the mixing around the front, but in this study the front velocity in a layer is calculated by averaging the velocities of the fluids on either side of the front. The averaged velocities seem to work as well as the rigorously calculated velocities.

\section{Effective Relative Permeabilities}

Two types of effective relative permeabilities can be generated depending on the voluine considered. The first type is an effective relative permeability for a cell, or flow unit as would be used in a numerical simulation. The second type is for a line normal to the main flow direction which we call the modified Hearn (1971) model.

Flow Unit Model - The effective relative permeability for a complete flow unit could be used in a numerical study with coarse grid to eliminate a spatial dimension of a problem or to reduce numerical dispersion. The flux of the injected fluid can be calculated by defining an apparent permeability as 


$$
Q_{1}=\frac{\tilde{k}_{x 1} H}{\mu_{1}}\left(\frac{\Delta_{x} \Phi}{\Delta x}\right)_{T}
$$

where 1 refers to the injected fluid and $T$ refers to the total potential gradient across the r.ross-section. The actual production of the injected fluid can be calculated from the summation of the preluction from the lavers that have broken through at a given time.

$$
Q_{1}=\frac{\sum_{i=1}^{N_{b}} \tilde{k}_{x i} h_{i}}{\mu_{1}}\left(\frac{\Delta_{x} \Phi}{\Delta x}\right)_{N_{b}}
$$

where $\mathrm{N}_{\mathrm{b}}$ refers to the number of the layer that has just broken through. Now the permeability to the injected fluid, in terms of the absolute permeability and the effective relative permeability, is

$$
\tilde{\mathrm{k}}_{\mathrm{x} 1}=\tilde{\mathrm{k}}_{\mathrm{x}} \tilde{\mathrm{k}}_{\mathrm{rl}}
$$

$\tilde{\mathrm{k}}_{\mathrm{x}}$ is the absolute effective permeability calculated as a function of layer thicknesses and apparent layer permeabilities and $\widetilde{\mathrm{k}}_{\mathrm{rl}}$ is the effective relative permeability to the injected fluid.

$$
H \tilde{k}_{x}=\sum_{i=1}^{N_{L}} \tilde{k}_{x i} h_{i}
$$

We substitute Eqs. (6), (7) and (8) into Eq. (5) to give

$$
\left(\tilde{k}_{r 1}\right)_{N_{b}}=\frac{\sum_{i=1}^{N_{b}} \tilde{k}_{x i} h_{i}}{\sum_{i=1}^{N_{L}} \tilde{k}_{x i} h_{i}} \frac{\left(\frac{\Delta_{x} \Phi}{\Delta x}\right)_{N_{b}}}{\left(\frac{\Delta_{x} \Phi}{\Delta x}\right)_{T}} \text { for } N_{b}=1, N_{L}
$$


where $\left(\frac{\Delta_{x} \Phi}{\Delta x}\right)_{N_{b}}$ is the potential gradient in the segment that has just broken-through, given by Eq. (3). The total potential drop, $\left(\frac{\Delta_{x} \Phi}{\Delta x}\right)_{T}$, must be recalculated after each layer breakthrough since it is a function of the front positions $x_{j}$ in each layer. These front positions are also needed for saturation calculations.

$$
x_{j}=\frac{L}{u_{b}} u_{j} \quad j=1, N_{L}+1
$$

where $x=L$ when $j=N_{L}+1$. The length of a segment is now $\Delta x_{j}=x_{j+1}-x_{j}$.

The potential drop across segment $\mathrm{j}$ is now

$$
\left(\Delta_{x} \Phi\right)_{j}=\frac{Q \Delta x_{j}}{\sum_{i=1}^{N_{L}} \frac{\tilde{k}_{x i} h_{i}}{\mu_{i j}}}
$$

and

$$
\left(\frac{\Delta_{\mathrm{x}} \Phi}{\Delta \mathrm{x}}\right)_{\mathrm{T}}=\frac{1}{\mathrm{~L}} \sum_{\mathrm{j}=1}^{\mathrm{N}_{\mathrm{L}}+1-\mathrm{N}_{\mathrm{b}}}(\Delta \mathrm{x} \Phi)_{\mathrm{j}}
$$

The effective relative permeabilities follow when Eq. (10) and Eq. (3) are substituted into Eq. (9).

The average saturation at break-through is calculated from the front positions in each layer.

$$
\left(S_{1}\right)_{N_{b}}=\left(i=1, N_{b}, \frac{(\phi h)_{i}}{H \bar{\phi}}\right)+\sum_{i=N_{b+1}}^{N_{L}} \frac{x_{i}(\phi h)_{i}}{H \bar{\phi}}
$$

assuming segregated flow in each layer.

Modified Hearn Model - In this section, we show how to generate effective relative permeabilities for layers with tensorial permeability when the grid cell volume 
approaches zero thickness. The procedure is a modification of the Hearn model for tensorial permeabilities. When the grid cell volume shrinks local and global potential gradients will be the same and Eq. (9) simplifies to

$$
\left(\tilde{k}_{r 1}\right)_{N_{b}}=\frac{\sum_{i=1}^{N_{b}} \tilde{k}_{x i} h_{i}}{\sum_{i=1}^{N_{L}} \tilde{k}_{x i} h_{i}} \quad \text { for } N_{b}=1, N_{L}
$$

The saturation at breakthrough of any layer is calculated from the summation of the thickness fractions of the layers that have broken through at a specified cross-section.

$$
\left(S_{1}\right)_{N_{b}}=\sum_{i=1}^{N_{b}} \frac{(\phi h)_{i}}{H \bar{\phi}}
$$

When we compare Eq. (9) with (12) and (10) with (13), we see that these two approaches give different results. In the flow unit model the effective relative permeabilities and saturations are functions of the volume considered, while in the modified Hearn model they are not. Yet another difference comes from the mobility ratio; in the modified Hearn model the effective relative permeability is not a function of mobility ratio. This result indicates that one should be very cautious when using the modified Hearn model in numerical simulations having large simulation cells.

In fact, when a numerical simulation cell is a small fraction of the flow unit, both methods give the same result. Figure 8 shows the calculated effective relative permeability for the injected fluid for various fractions of the original flow unit. The thickness and length of the original flow unit were (10 and $100 \mathrm{ft}) 3$ and $30 \mathrm{~m}$, respectively. In all effective relative permeability calculations, we applied the boundary conditions to the boundary of the flow unit not to the particular simulation cell boundary. The effective relative permeabilities for the flow unit are strong functions of size when the cell volume is a comparable fraction of the flow unit volume. For the particular flow unit in Fig. 8 if the cell volume is less than $40 \%$ of the original flow unit, the scale dependency is negligible. 
The effective relative permeability curves in Fig. 8 initially exhibit a phenomenon that is normally seen only in immiscible displacements. Until the saturation of injected fluid reaches a specific value, called the average saturation behind the front in immiscible displacements, the injected fluid will not be produced. The apparent residual saturation in Fig. 8 implies that the saturation of the injected fluid in the finite flow unit volume must reach a non-zero value before any injected fluid is produced. This result is expected, since the effective relative permeability calculation must account for vertical sweep efficiency ever before breakthrough. The discontinuous initial increase in effective relative perrneability (from zero to a value) stems from the specific layering used in this example. The shape of the curve beyond the initial discontinuity depends on the specific geology of the flow unit.

Thu relative permeability to the resident fluid 2 is the complement of the relative permeability to fluid 1 .

\section{Comparison with Numerical Calculations}

The finite element simulator was run for single-phase flow in a eight-layer crosssection where each layer has tensorial permeability. To avoid excessive dispersion (numerical and physical) a front tracking method was used once the pressure field was known (this was also used in Figs. $5 \mathrm{~d}$ and $6 \mathrm{~d}$ ). The numerical effective permeability is calculated from the velocities for each layer at the producing end. The overall production of the injected fluid is written as a function of the apparent permeability of the permeable medium to that fluid.

$$
H \tilde{k}_{x}=\sum_{i=1}^{N_{L}} h_{i}\left(k_{x x i}-\frac{k_{x y i}^{2}}{k_{y y i}}\right)
$$

and the effective relative permeability follows from 


$$
\tilde{k}_{r 1}=\frac{\mu_{1} \sum_{i=N_{b}+1}^{N_{L}} h_{i} u_{i}}{\left(\frac{\Delta_{x} \Phi}{\Delta x}\right) r \sum_{i=1}^{N_{L}} h_{i}\left(k_{x x i}-\frac{k_{x y i}^{2}}{k_{y y i}}\right)}
$$

Equation (14) gives numerically calculated effective relative permeabilities. The corresponding saturations are calculated by Eq. (10).

Figure 9a compares the analytirally and numerically calculated effective relative permeabilities for a displacement having a mobility ratio of 10 . The aspect ratio of the cross-section was also 10 . The figure shows an good match between two methods. Figure $9 \mathrm{~b}$ shows that the recovery predictions from both the numerical and analytical methods also compare very well. From these results we conclude that the proposed analytical method of calculating effective relative permeabilities can capture the fluid flow characteristics of the cross-bedded flow units.

The calculated analytical effective relative permeabilities are very strong functions of the mobility ratio. Figure 10 shows analytically calculated effective relative permeabilities for the mobility ratios of 1 and 10. The figure shows a large difference between the relative permeabilities. This result is somewhat expected, because these effective relative permeabilities are also indicators of the average saturation at breakthrough of the referred displacements. The sweep efficiency, on the other hand, is expected to be a function of the mobility ratio which is also reflected in Fig. 10.

Even though we appreciate the generality of VE approach (the VE assumption can greatly simplify oil recovery calculations), the entire procedure is restricted to the crosssections having a large aspect ratio. Figure 11 shows the analytical and numerical effective relative permeabilities for the aspect ratio of 1 . The match between the two effective relative permeabilities is poor. The only difference between the conditions under which the effective relative permeabilities in Figs. 11 and $9 \mathrm{a}$ are generated is the aspect ratio. The aspect ratio for the cross-section for which the effective relative permeabilities in Fig. 11 are generated was $1[10 \times 10 \mathrm{ft}(3 \times 3 \mathrm{~m})]$. while for the one in Fig. 9a was $10[100 \times 10 \mathrm{ft}$ $(30 \times 3 \mathrm{~m})]$. Comparing Figs. $9 \mathrm{a}$ and 11 we see that the analytical effective relative permeabilities are insensitive to the aspect ratio (the length is changed bere), where the numerical effective relative permeability is a function of the aspect ratio. Although the aspect ratio of the simulation cell is a restriction for the VE approach, it may not be a 
significant drawback, since we have some liberty of choosing the dimensions of the numerical grids. From these results we conclude that for the flow unit we studied here the aspect ratio of 10 is a good measure of the condition in which VE applies.

\section{Application to Large-Scale Simulations}

The next task is to apply the analytical method to a large-scale simulation study to show that the method can be used to reduce computational burden. First we simulate flu flow in the Page Sandstone outcrop flow field (C2 and B units of Northeast wall) where ail the details of the permeability field are included, then repeat the simulations with a coarse numerical grid scheme using the effective relative permeabilities generated particularly for $\mathrm{C} 2$ and $\mathrm{B}$ units, to repeat the recovery predictions of detailed simulations. See subtask $1 \mathrm{~d}$ for a discussion on the detailed simulations.

We intend to repeat the results of the fine grid simulations by applying the analytic method to one-dimensional simulations of fluid flow in the $\mathrm{C} 2$ and B units of the Page Sandstone with less detail. For simplicity the flow field is discretized into 36 elements for a one-dimensional simulation study, instead of 11520 elements of the detailed simulations. The one-dimensional simulation conditions are similar to the conditions of the modified Hearn model. The same effective relative permeability curve (modified Hearn model) was used for all elements. The recovery predictions from the detailed simulation runs with 11520 elements and from the simulations (one-dimensional 36 elements) with analytically generated effective relative permeabilities are compared in Fig. 12. The recovery predictions agree well for both mobility ratios 1 and 10 , although the detailed simulation runs cost 1000 times more than one-dimensional simulation runs. The comparisons of the recovery predictions indicate that the calculated effective relative permeabilities can capture the effect of heterogeneity on vertical sweep efficiency.

\section{Conclusions}

This work extends the Hearn method for calculating effective relative permeabilities in viscous-dominated displacements to heterogeneous cross-bedded media. The specific curves generated are valid for eolian sands, but the procedure should be general for any cross-bedded medium, although, strictly speaking, it is restricted to single beds. Specific conclusions are,

1. Vertical equilibrium applies to cross-bedded layers, just as it does to isotropic layers, if the aspect ratio of the medium is large. Cross-bedding does change the zero-potential gradient direction compared to isotropic media. 
2. An anisotropic permeability tensor is a good representation of cross-bedding as long as the size of the flow unit is more than about 10 times greater than the crossbed spacing. This conclusion applies to both the potential distribution and the shape and location of the displacement front.

3. Multiple parallel layers of anisotropic media behave very much like multiple layers of isotropic media. In the first case, each layer must be assigned an effective permeability which is a function of the elements of the permeability tensor. Crossbedding by itself does not cause fluid crossflow.

4. The effective relative permeabilities are functions of mobility ratio and flow unit size. If the latter shrinks to a vertical line, the effective relative permeabilities become the same as in the Hearn procedure where they depend on neither the mobility ratio nor the flow unit size.

5. The method is applied to one-dimensional simulations of fluid flow in the $\mathrm{C} 2$ and $\mathrm{B}$ units of the Page Sandstone with less detail (36 elements, instead of 11520 elements of the detailed simulations). The resulting recovery predictions for different mobility ratios are compared with the ones from the detailed simulations. The comparisons of the recovery predictions indicate that the calculated effective relative permeabilities can capture the effect of heterogeneity on sweep efficiency.

The effective relative permeabilities generated are for two-dimensional miscible displacements in a single eolian cross-bed under conditions where gravity and dispersion were negligible. These restrictive considerations are offset by the considerable geologic information imbedded in the functions.

\section{References}

Bear, J.: Dynamics of Fluids in Porous Media, American Elsevier Publishing Co. Inc., New York City, (1977), Chap. 5.

Chandler, M. A.: Depositional Controls on Permeability in an Eolian Sandstone Sequence Page Sandstone. Northern Arizona, M.A. Thesis, The University of Texas at Austin, December, 1986.

Coats, K. H., J. R. Dempsey, and J. H. Henderson: "Use of Vertical Equilibrium in Two-Dimensional Simulation of Three-Dimensional Reservoir Performance," Society of Petroleum Engineers Journal (March 1971), 63-71. 
Coats, K. H., R. L. Nielsen, M. H. Terhune, and A. G. Weber: "Simulation of ThreeDimensional, Two-Phase Flow In Oil and Gas Reservoirs," Society of Petroleum Engineers Journal (December 1967), 377-388.

Davies, B. J. and H. H. Haldorsen: "Pseudofunctions in Formations Containing Discontinuous Shales: A Numerical Study," SPE 16012, presented at the 1987 Ninth SPE Symposium on Reservoir Simulation in San Antonio, TX, February 14.

Goggin, D. J., M. A. Chandler, G. A. Kocurek, and L. W. Lake: "Patterns of Permeability in Eolian Deposits," Society of Petroleum Engineers Formation Evaluation (June 1988), 297-306.

Greenkorn, R. A., C. R. Johnson, and L. K. Shallenberger: "Directional Permeability of Heterogeneous Anisotropic Porous Media," Society of Petroleum Engineers Journal (June 1964), 124-132.

Hawthorne, R. G.: "The Effect of Capillary Pressure in a Multilayer Model of Porous Media," Society of Petroleum Engineers Journal (December 1975), 467-476.

Hearn, C. L.: "Simulation of Stratified Waterflooding by Pseudo Relative Permeability Curves," Journal of Petroleum Technology (July 1971), 805-813.

Hewett, T. A. and R. A. Behrens: "Scaling Laws in Reservoir Simulation and Their Use in a Hybrid Finite Difference/Streamtube Approach to Simulating the Effects of Permeability Heterogeneity," presented at the 1989 Second International Reservoir Characterization Technical Conference, Dallas, June 25-28.

Jacks, H. H., O. J. E. Smith, and C. C. Mattax: "The Modeling of a Three-Dimensional Reservoir with a Two-Dimensional Reservoir Simulator - The Use of Dynamic Pseudo Functions," Society of Petroleum Engineers Journal (June 1973), 175-185. Kasap, E.: "Analytic Methods to Calculate an Effective Permeability Tensor and Effective Relative Permeabilities for Cross-Bedded Flow Units," Ph.D. Dissertation, The University of Texas at Austin, May, 1990.

Kasap, Ekrem and Larry W. Lake: "An Analytic Method to Calculate the Effective Permeability Tensor of a Grid Block and Its Application in an Outcrop Study," Society of Petroleum Engineers Formation Evaluation, in press, 1990.

Killough, J. E. and H. P. Foster, Jr.: "Reservoir Simulation of the Empire Abo Field: The Use of Pseudos in a Multi-layered System," Society of Petroleum Engineers Journal (October 1979), 279-288.

Knight, J. B.: Eolian Bedform Reconstruction: A Case Study from the Page Sandstone, Northern Arizona, M.A. Thesis, The University of Texas at Austin, May, 1986. 
Kortekaas, T. F. M.: "Water Oil Displacement Characteristics in Cross-Bedded Reservoir Zones," SPE 12112, presented at the 1983 SPE 58th Annual Technical Conference. and Exhibition in San Francisco, CA, October 5-8.

Kossack, C. A., J. O. Aasen, and S. T. Opdal: "Scaling-Up Laboratory Permeabilities and Rock Heterogeneities with Pseudo Functions for Field Simulations," SPE 18436, presented at the 1989 10th SPE Symposium on Reservoir Simulation Symposium in Houston, TX, February 6-8.

Kyte, J. R. and D. W. Berry: "New Pseudo Functions to Control Numerical Dispersion," Society of Petroleum Engineers Journal (August 1975), 269-276.

Lake, L. W.: Enhanced Qil Recovery Prentice Hall, Inc. Englewood Cliffs, New Jersey, (1989).

Lake, L. W.: "The Origins of Anisotropy," Journal of Pet. Tech., (April 1988), 395-396.

Lake, Larry W., E. Kasap, and M. Shook: "Pseudofunctions - The Key to Practical Use of Reservoir Description," presented at the 1989 Second International Conference on North Sea Oil and Gas Reservoirs in Trondheim, Norway, May 8-11.

Lasseter, T. J., J. R. Waggoner, and L. W. Lake: "Reservoir Heterogeneities and Their Influence on Ultimate Recovery," in Reservoir Characterization edited by Larry W. Lake and Herbert B. Carroll, Jr., Academic Press, Inc., New York, (1986), 545559.

Martin, J. C.: "Partial Integration of Equation of Multiphase Flow," Society of Petroleum Engineers Journal (December 1968), 370-380.

Muggeridge, A. H. "Generation of Pseudo Relative Permeabilities from Detailed Simulation of Flow in Heterogeneous Porous Media," presented at the 1989 Second Irternational Reservoir Characterization Technical Conference, Dallas, June 25-28.

Muskat, M.: The Flow of Homogeneous Fluids Through Porous Media, J. W. Edwards Inc., Ann Arbor, Michigan, (1946).

Rosenzweig, J. J., N. A. Abdelmalek, and J. R. Gochnour: "The Development of Pseudo Functions for Three Phase Black Oil Simulators," In Reservoir Characterization edited by Larry W. Lake and Herbert B. Carroll, Jr., Academic Press, Inc., New York, (1986), 393-443.

Scheidegger, A. E.: The Physics of Flow through Porous Media, University of Toronto Press, Toronto, (1972).

Starley, G. P.: "A Material Balance Method for Deriving Interblock Water/Oil Pseudofunctions for Coarse Grid Reservoir Simulation," SPE 15621, presented at 
the 1986 SPE 61st Annual technical Conference and Exhibition in New Orleans. LA, October 5-8.

Thomas, G. W.: "An Extension of Pseudofunction Concepts," SPE 12274, presented at the 19837 th SPE Symposium on Reservoir Simulation in San Francisco, CA, November 15-18.

van Pollen, H. K., E. A. Breitenbach, and D. H. Thurnan: "Treatment of Individual Wells and Grids in Reservoir Modeling," Society of Petroleum Engineers Journal (December 1968), vol. 8, 341-346.

Young, G. R.: "Determining Permeability Anisotropy from a Core Plug using a Minipermeameter," M.S. Thesis, The University of Texas at Austin, May, 1989.

Zapata, V. J. and L. W. Lake: "A Theoretical Analysis of Viscous Crossflow," SPE 10111, presented at the 1981 56th Annual Fall Technical Conference and Exhibition of the Society of Petroleum Engineers in San Antonio, TX, October 5-7.

\section{Nomenclature}

\begin{tabular}{|c|c|}
\hline \multicolumn{2}{|c|}{$\mathrm{L}=$ length, $\mathrm{t}=$ time, $\mathrm{F}=$ force, $\mathrm{m}=$ mass; $[=]$ means "has units of" } \\
\hline h & Thickness of the subunit (cross-bedded layer) $[=] \mathrm{L}$ \\
\hline$h_{c b}$ & Thickness of the cross bed $[=] \mathrm{L}$ \\
\hline $\mathrm{H}$ & Thickness of a flow unit $[=] \mathrm{L}$ \\
\hline$k_{n}$ & Permeability in the direction normal to cross-bedding [ $=] \mathrm{L}^{2}$ \\
\hline$k_{\mathrm{p}}$ & Permeability in the direction parallel to cross-bedding $[=] \mathrm{L}^{2}$ \\
\hline $\mathrm{k}_{\mathrm{T}}$ & Relative permeability \\
\hline $\mathrm{L}$ & Length of a flow unit $[=] \mathrm{L}$ \\
\hline M & Mobility ratio (displacing/displaced) \\
\hline$N_{b}$ & The number of the layer that just broke through \\
\hline$N_{L}$ & Total number of layers \\
\hline Q & Flow Rate $[=] \mathrm{L}^{3} / \mathrm{t}$ \\
\hline S & Saturation \\
\hline $\mathrm{x}, \mathrm{y}, \mathrm{z}$ & Simulation coordinate system $[=] \mathrm{L}$ \\
\hline $\mathrm{u}$ & Darcy or superficial velocity $[=] \mathrm{L} / \mathrm{t}$ \\
\hline $\mathrm{V}$ & Volume $|=| L^{3}$ \\
\hline
\end{tabular}




$\begin{array}{ll}\alpha_{c b} & \text { Treek } \\ \Delta & \text { Cross-bedding angle (co'inter-clockwise from vertical) } \\ \phi & \text { Differences } \\ \Phi & \text { Porosiiy } \\ \mu & \text { Potential }[=] \mathrm{F} / \mathrm{L}^{2} \\ \rho & \text { Viscosity }[=] \mathrm{F} / \mathrm{L}-\mathrm{t} \\ & \text { Density }[=] \mathrm{m} / \mathrm{L}^{3} \\ & \\ \mathrm{~b} & \text { Subscripts } \\ \mathrm{i} & \text { Breakthrough } \\ \mathrm{T} & \text { Layer or component number } \\ \mathrm{x}, \mathrm{y} & \text { Total } \\ 1 & \text { Simulation coordinates } \\ 2 & \text { Injected fluid } \\ & \text { Produced fluid } \\ & \\ \sim & \text { Superscripts } \\ \rightarrow & \text { Effective } \\ - & \text { Vector }\end{array}$


Table 1: Parameters Assigned to the Layers

\begin{tabular}{|l:l|l|l|}
\hline Laver \# & \% Thickness & $\alpha$ (degree) & $\%$ Cross-bed Thickness \\
\hline 1 & 12.5 & 15 & 5 \\
\hline 2 & 10 & 20 & 8 \\
\hline 3 & 7.5 & 28 & 13 \\
\hline 4 & 7.5 & 34 & 17 \\
\hline 5 & 10 & 38 & 26 \\
\hline 6 & 10 & 45 & 43 \\
\hline 7 & 12.5 & 60 & 71 \\
\hline 8 & 30 & 90 & 100 \\
\hline
\end{tabular}




\section{List of Figures}

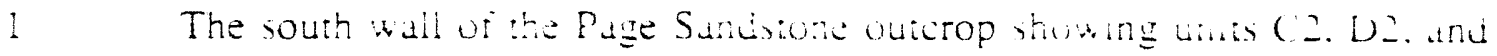
D4. The thicknesses of units D2 and D4 are approximately $10 \mathrm{ft}(3 \mathrm{~m})$.

$2 \quad$ A model $[10 \times 25 \mathrm{ft}(3 \times 8 \mathrm{~m})$ ] for unit D4

(a) The dark and the light areas are windripple and graintlow itratification types, respectively.

(b) A set of uniform!y cross-bedded parallel layers which is an approximation to the model.

3 Schematic iliustration of a cross-bedded section. The shows the cross-bed angle. large-(light) and small-permeability (dark) regions and the principa! directions of the permeabiliry ( $n$ and $p$ ).

$4 \quad$ Apparent permeabilities (Eq. 1 ) in the horizontal direction after the faralle! layers are homogenized. See Table 1 for the data used.

$5 \quad$ Cross-bedding and comparison of isopotential lines in a single laver.

(a) Large llight) and small (dark) permeability streaks of iross-beduing $\mathrm{L} / \mathrm{H}=5 . \alpha_{c b}=\pi / 4 . \mathrm{k}_{1} / \mathrm{k}_{2}=10 . \mathrm{h}_{1} / \mathrm{h}_{2}=1$ ) and tinite element grid scheme (lines not seen on the dark streaks).

(b) Isopotential lines in the cross-bedded system (contour interval CI=2\% of the applied potential difference).

(c) Isopotential lines $(\mathrm{CI}=2 \%)$ in the homogenized medium.

(d) Comparison of displacement fronts at different injection times.

6 Cross-bedding and comparison of isopotential lines in three parallel layers.

(a) Cross-bedding (from top to bottom $\alpha_{c b}=\pi / 6, \alpha_{c b}=\pi / 3$ and $\alpha_{c b}=$ $3 \pi / 4, \mathrm{~L}, \mathrm{H}=5, \mathrm{k}_{1} / \mathrm{k}_{2}=10, \mathrm{~h}_{1} / \mathrm{h}_{2}=1$, and finite element grid scheme lines not seen on the dark streaksi.

(b) Isopotential lines in the cross-bedded system $\left(\mathrm{CI}=2 C_{C}\right.$ of the applied potential differencel.

(U) Isopotential lines $(\mathrm{CI}=2 \%$ i in the homogenized medium.

(d) Comparison of displacement fronts at different injection times.

- Schematc illustration of a ystem in vertical equilibrium.

(a) The front postions in each layer and the segments with constant potential gradient.

(b) Horizontal distribution of the now potential. 
8 Effective relative permeabilities as a function of injected fluid saturation for different sizes of the nurnerical simulation cells, $M=10$.

9 Comparison between the analytical and numerical methods at $M=10$.

(a) Effective relative permeability of displacing fluid.

(b) Fractional recoveries of resident fluid.

10 Comparison of the effective relative permeabilities calculated by the analytical method (VE) for mobility ratios of 1 and 10 .

11 Comparison of the effective relative permeabilities calculated by the analytical (VE) and numerical methods for an aspect ratio of 1 .

12 Comparison of recoveries calculated from the explicit numerical simulation of $\mathrm{B}$ and $\mathrm{C} 2$ units and one-dimensional coarse grid simulations by using effective relative permeabilities generated for $\mathrm{B} 2$ and $\mathrm{C}$ units.
(a) $\mathrm{M}=1$
(b) $\mathrm{M}=10$ 


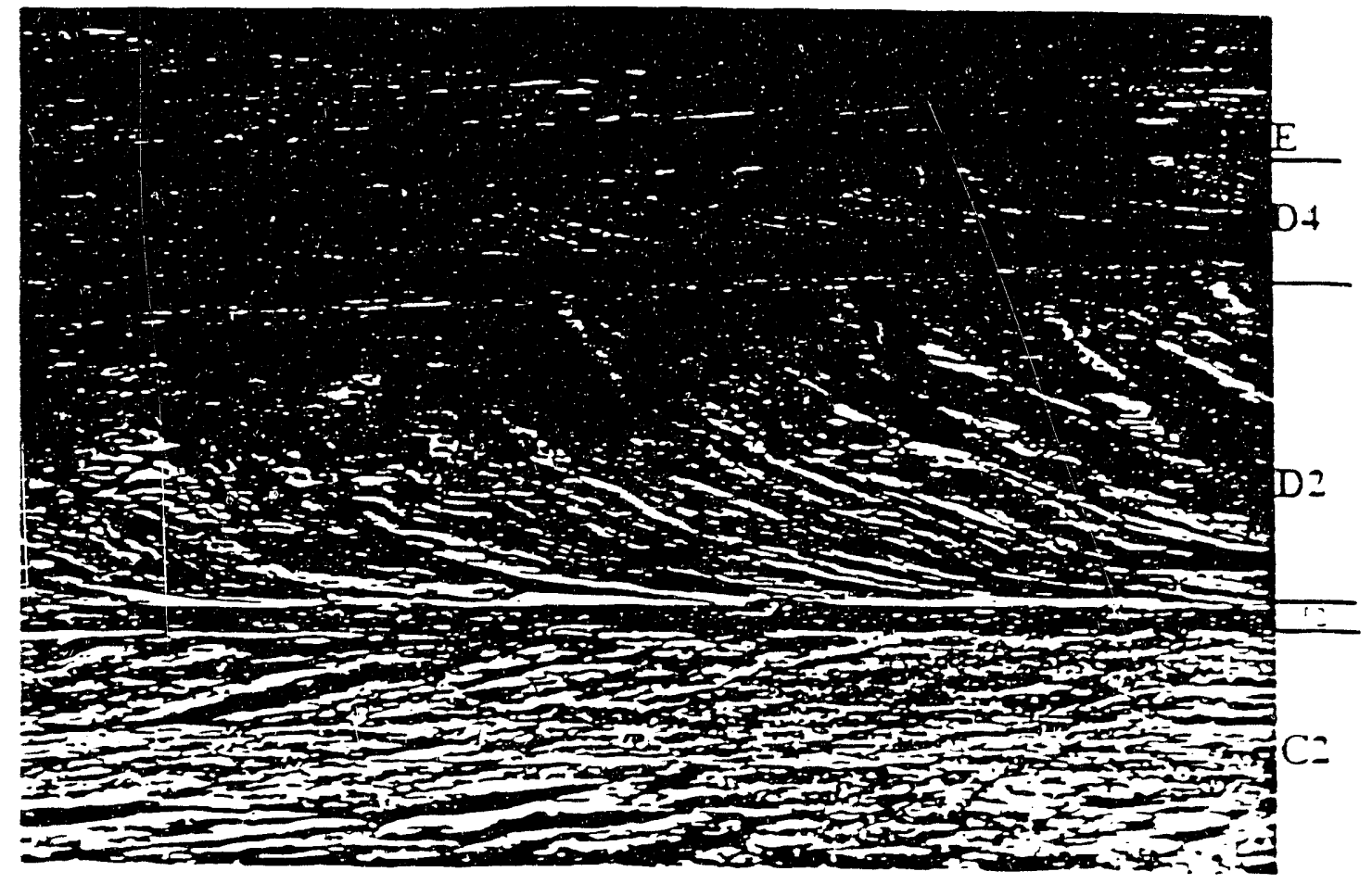

Ėg.: The south wail of the Page Sancistone outcrop showing units C2. D2. and D4. The thisisesses of units D2 and D4 are approximately loft (三ت). 


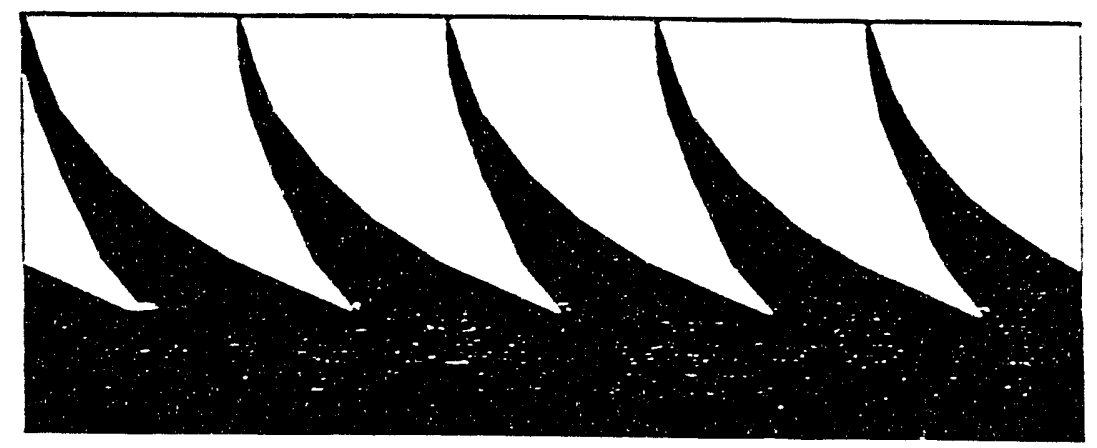

(a)

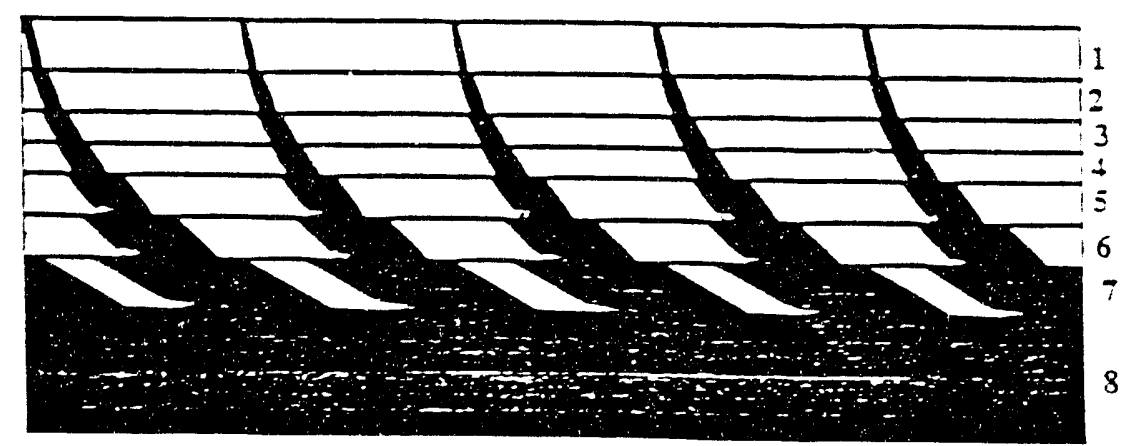

(b)

Fig. 2 A rodel $[10 \times 25 \mathrm{ft}(3 \times 8 \mathrm{~m})]$ for unit $D 4$.

(a) The dark and the light areas are windippie and granfiow szatiscation types, respectiveiy.

(o) A set of urfor-ay cross-becied parallel layers which is an approxization to the model 
L

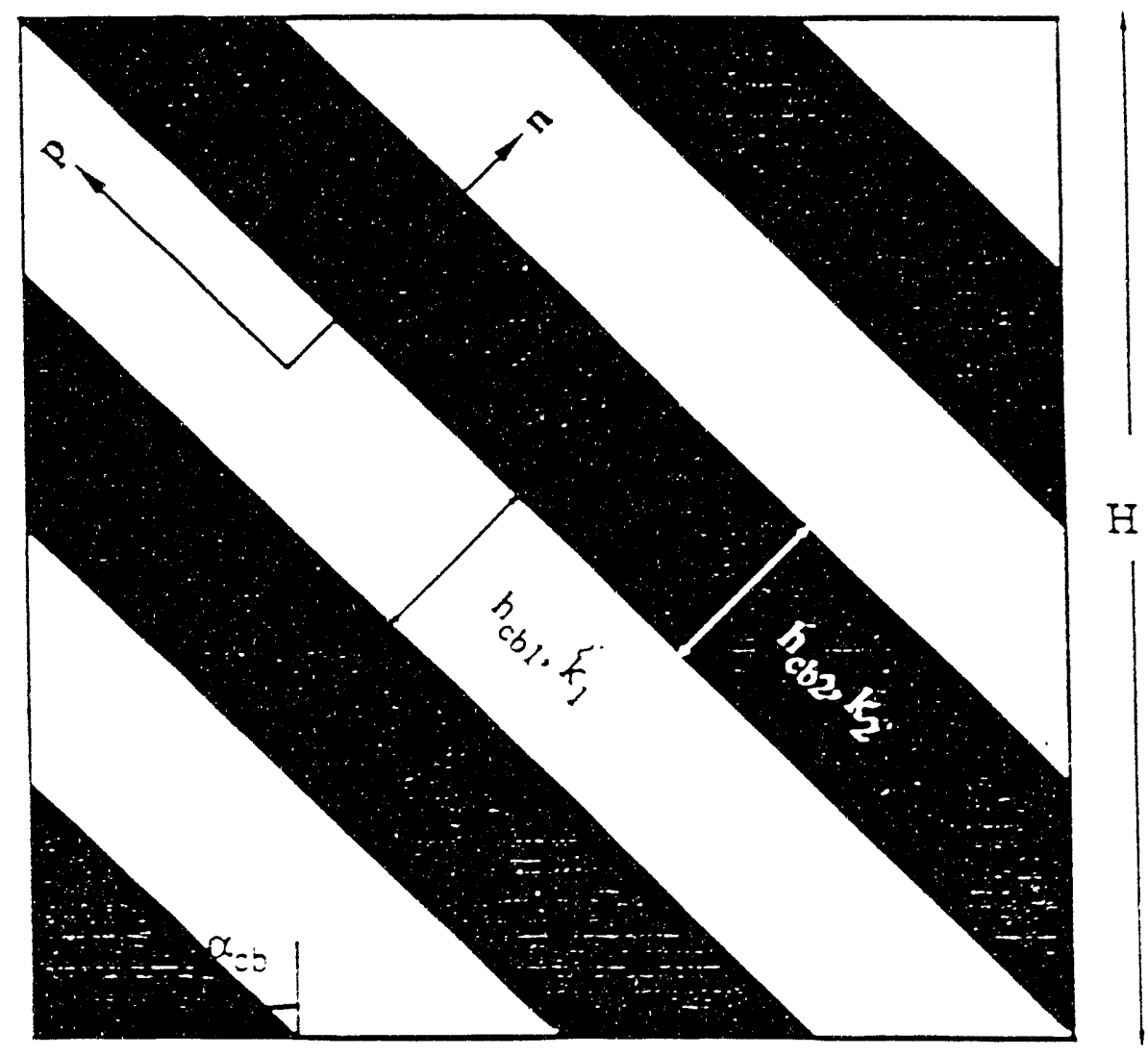

Fig. Z Screzatic ibustation of a cross-bedied section. The shows the cross-oed angie,

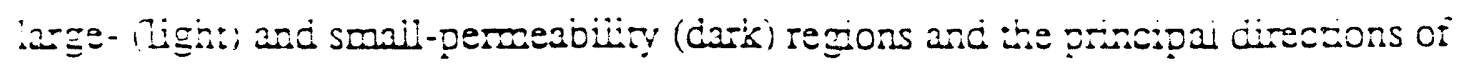
the perseabiity (n and p). 


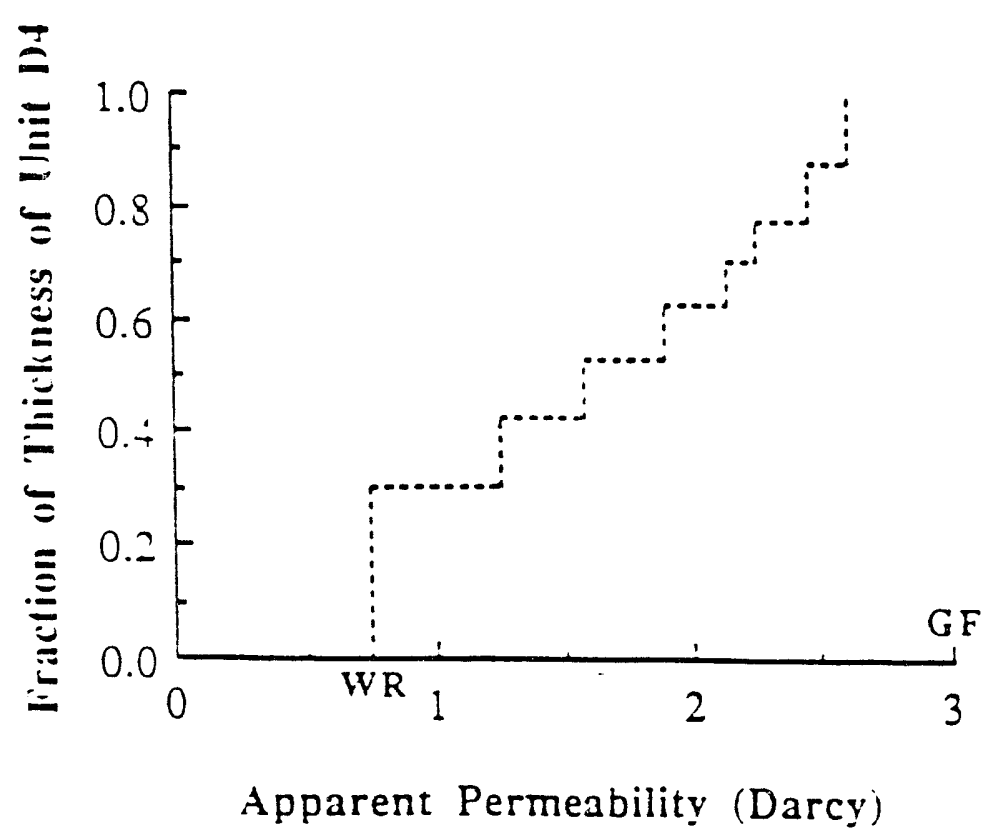

Fig. 4 Apparent permeabilities (Ec. 1) in the horizontal direction after the parallel layers are homogenized. See Table 1 for the data used. 


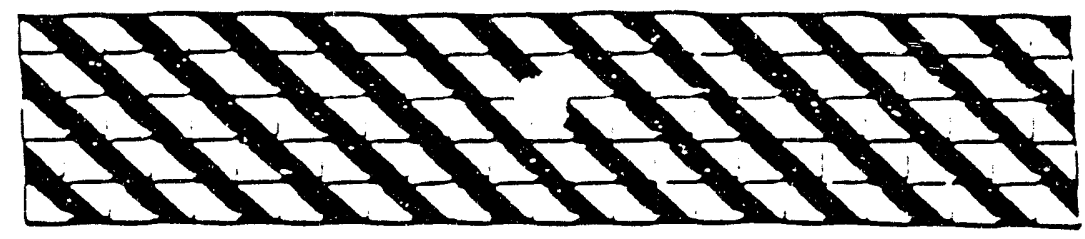

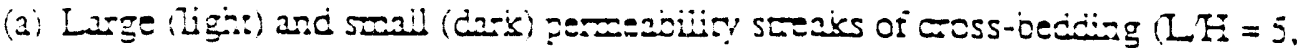

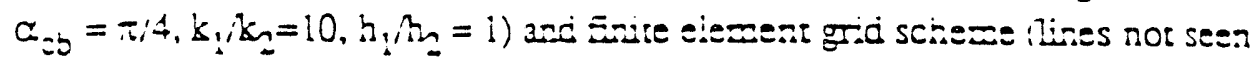
on the daris steaksi.

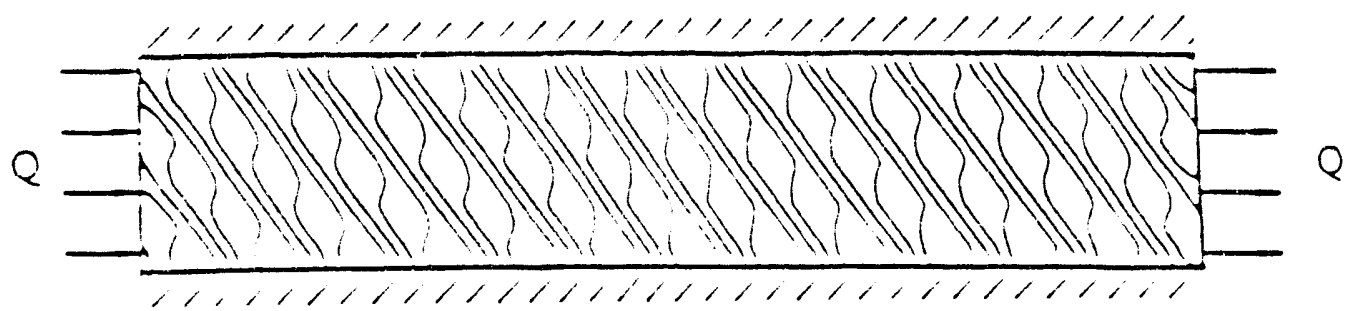

(b) Isopoten:al lines in the coss-becided syste= (contour in:erval $C=2 \%$ of the

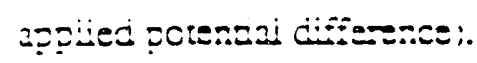

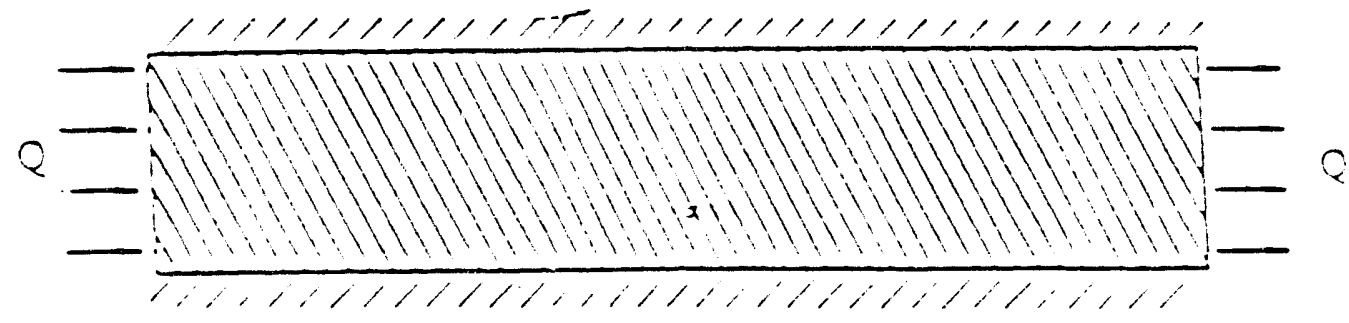

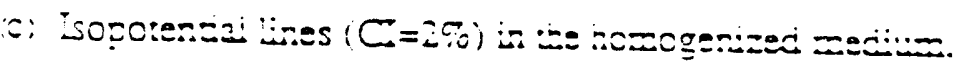

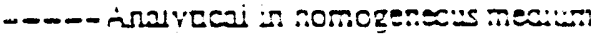

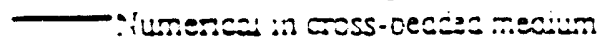

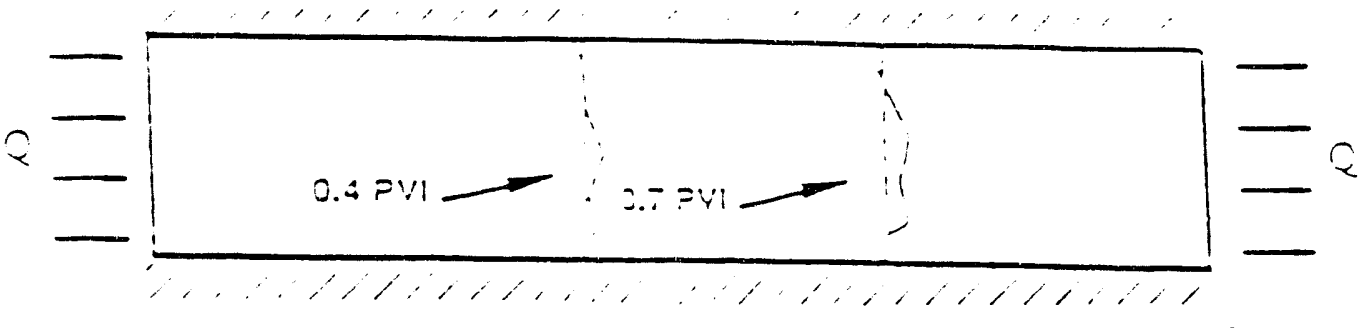

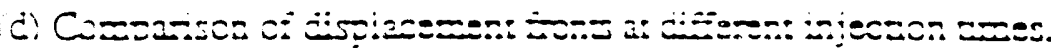

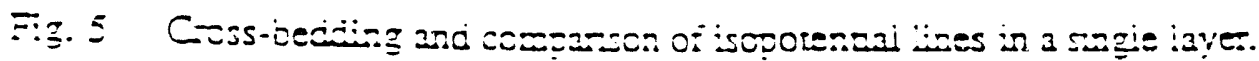


(a)

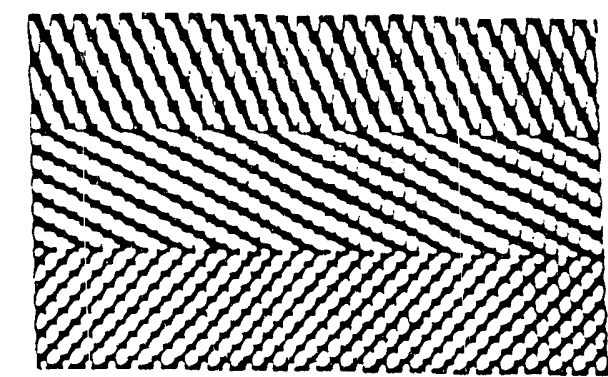

(b)

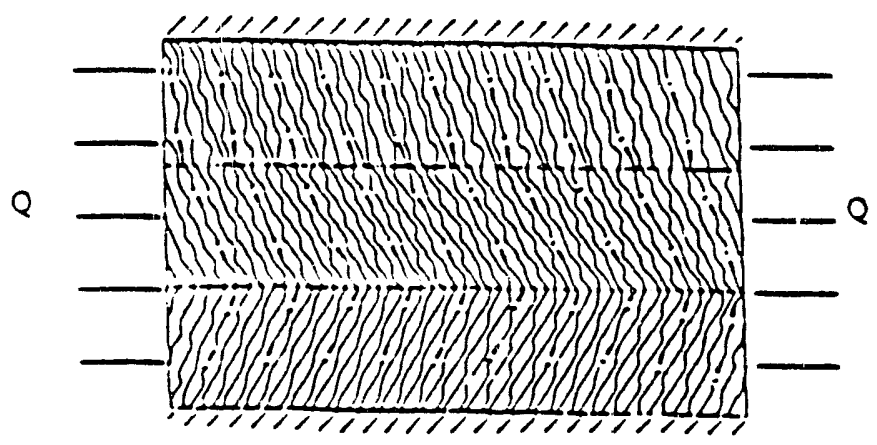

(c)

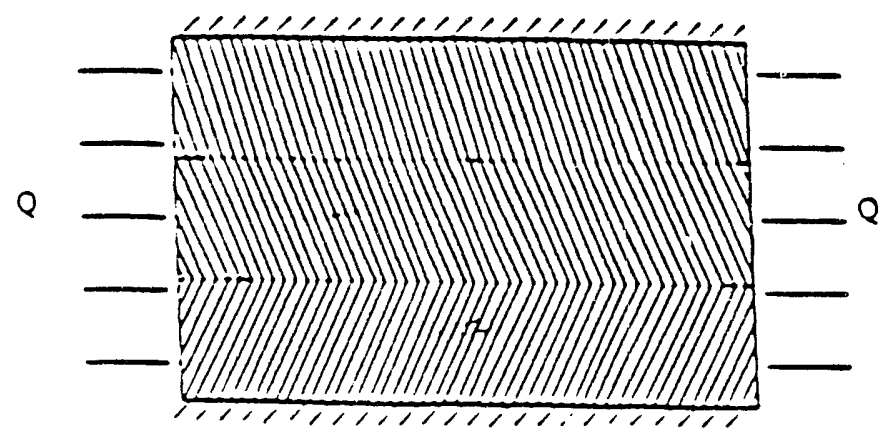

(d)

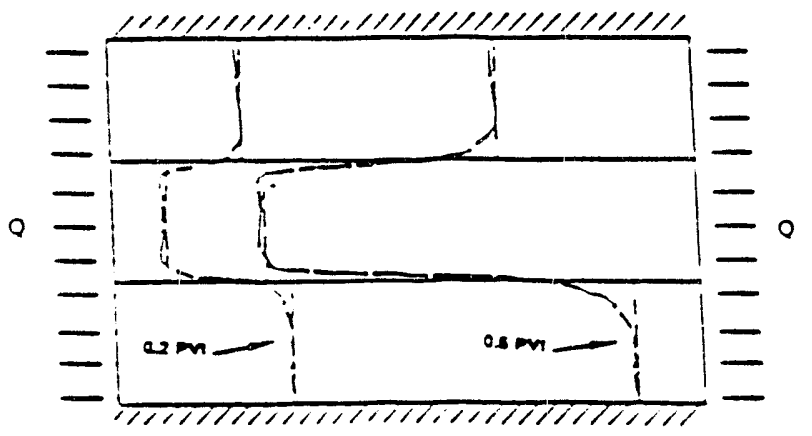

Fig. 6 Cross-bedding and companson of isopotencial lines in three parallel layers.

(a) Coss-bedding ( from top to bottom $\alpha_{i b}=\pi / 6, \alpha_{i b}=\pi \beta$ and $\alpha_{i b}=3 \pi / 4$, $\left.\mathrm{L} / \mathrm{H}=5, k_{1} / \mathrm{k}_{2}=10, h_{1} / h_{2}=1\right)$ and finie element grid scheme (lines not seen on the dark streaks).

(b) Isopotentail lines in the cross-bedded system ( $\mathrm{CI}=2 \%$ of the appiied porencal differesce!.

(c) Isopotenzai lires ( $\mathrm{CI}=2 \%$ ) is the hoxogenized medium

(d) Companson of ¿ispiacerent zonts at different injecton di=es. 


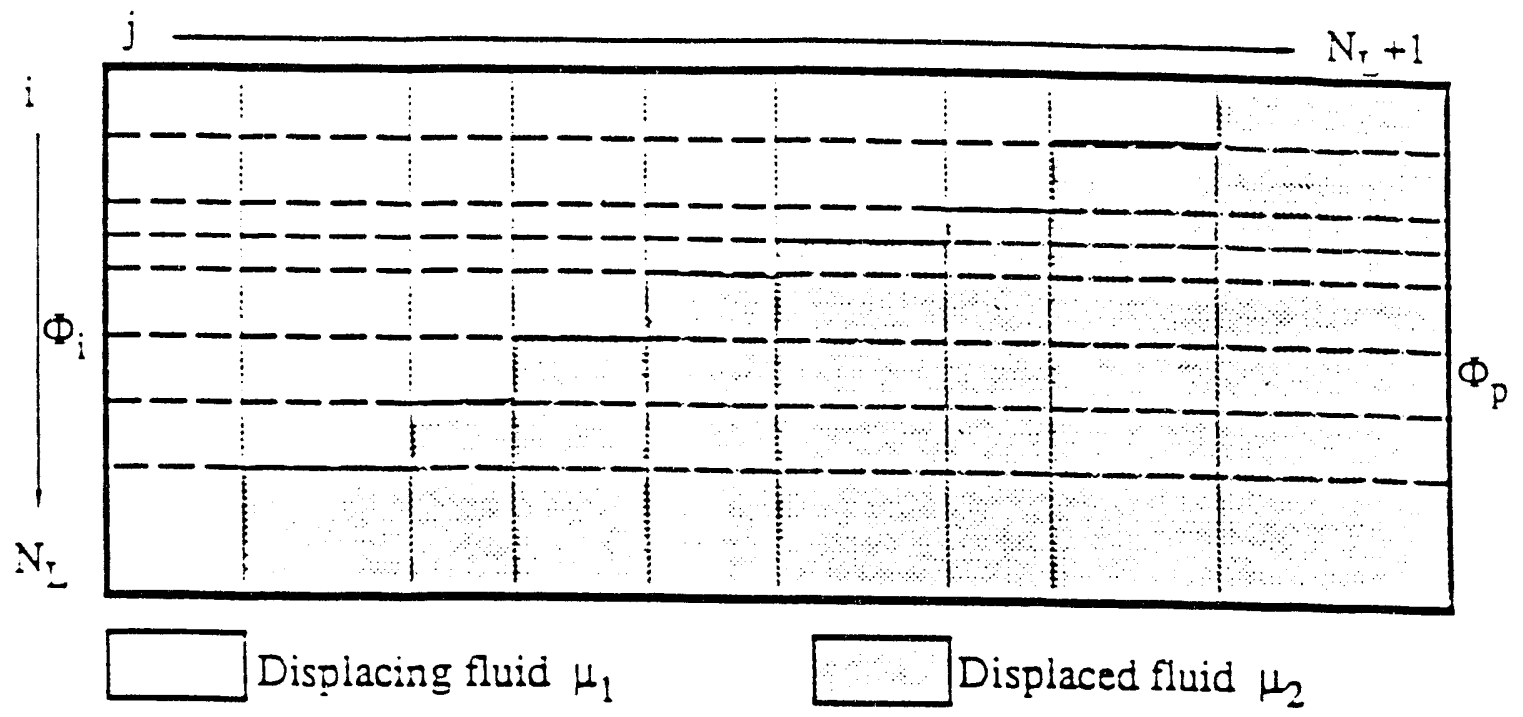

(a)

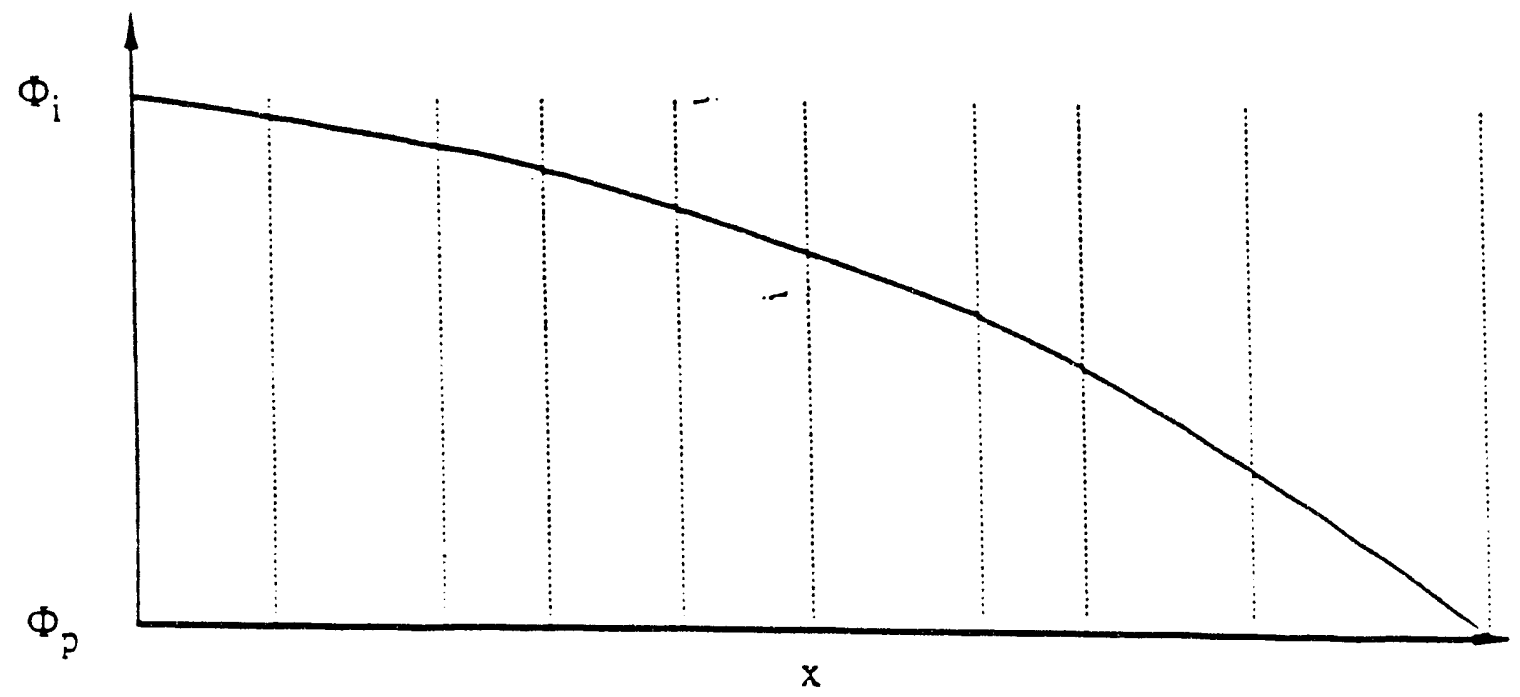

(b)

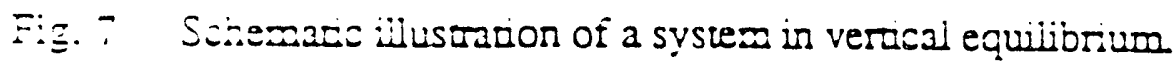

(a) The tont positions in each layer and the segments with consiant potential gradient.

(b) Horizontal distribucion of the Ilow potencial. 


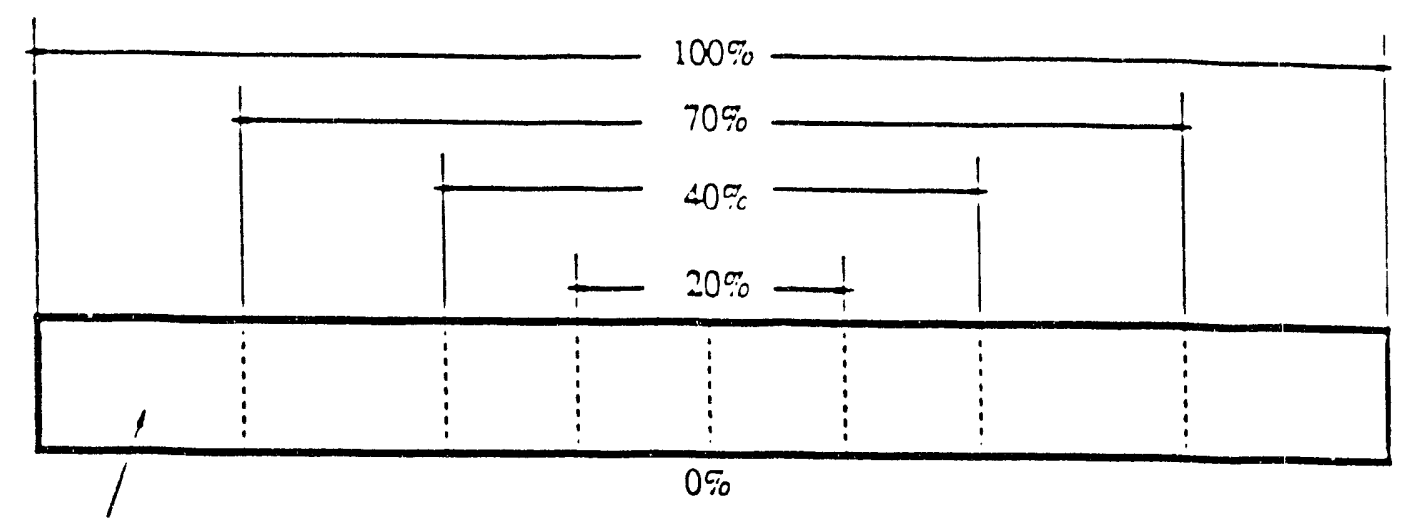

Flow unit

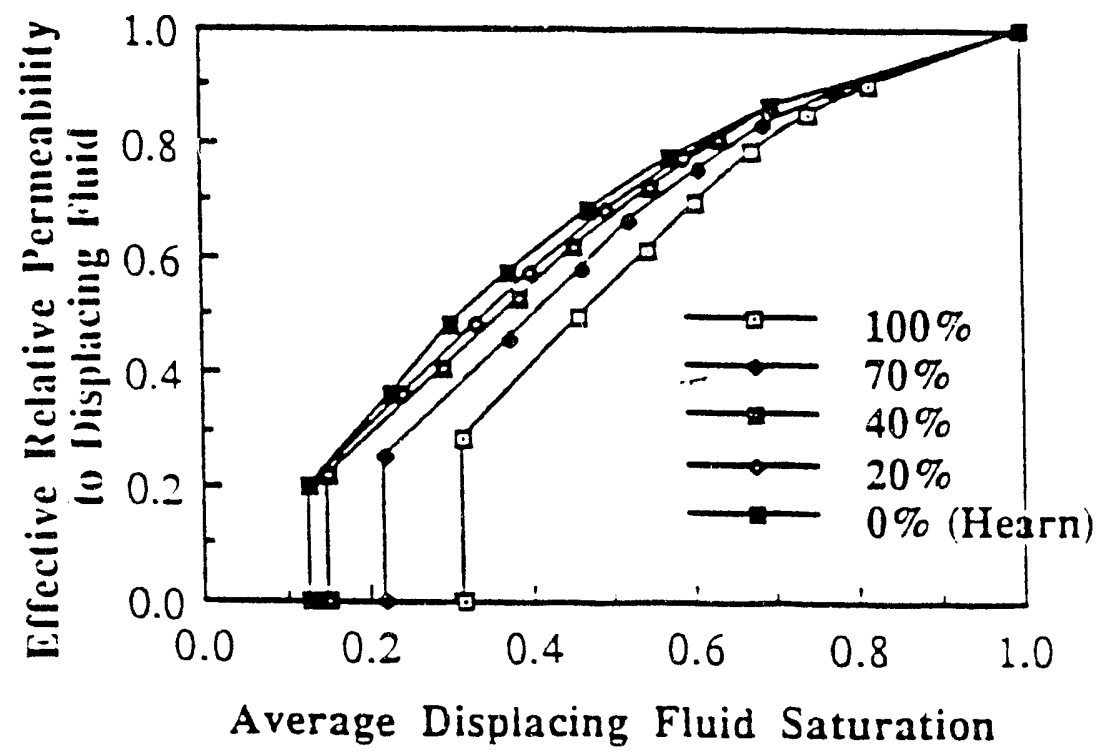

Fig. 8 Effective relative permeabilities as a function of injected fluid saturation for different sizes of the numerical simulation cells, $M=10$. 


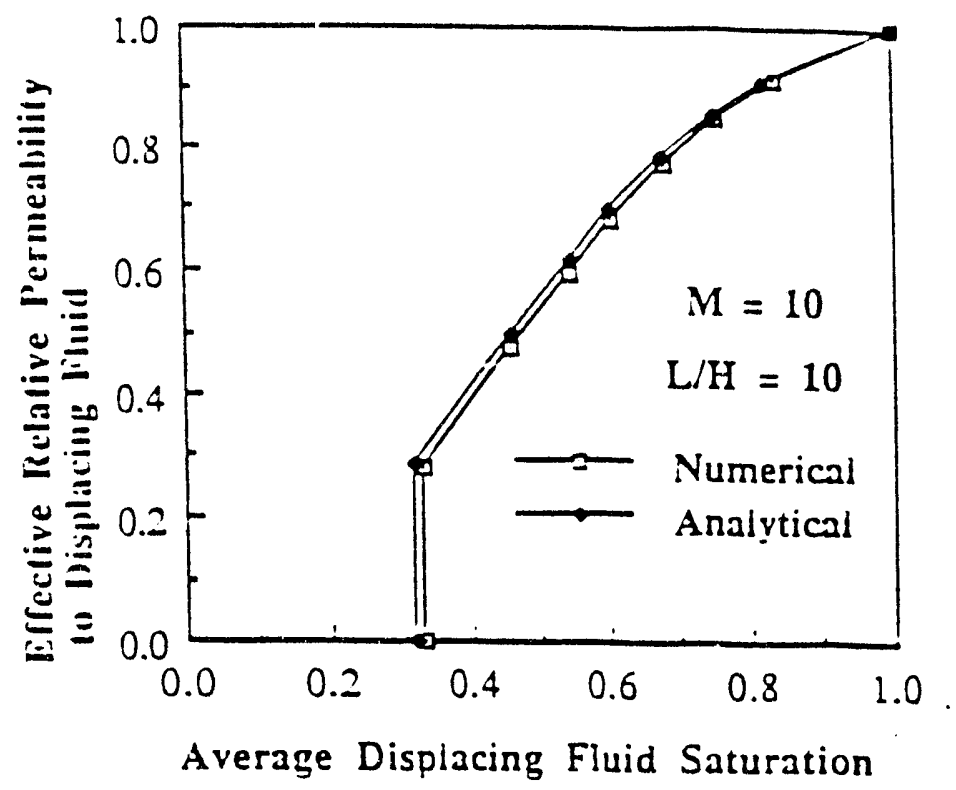

(a)

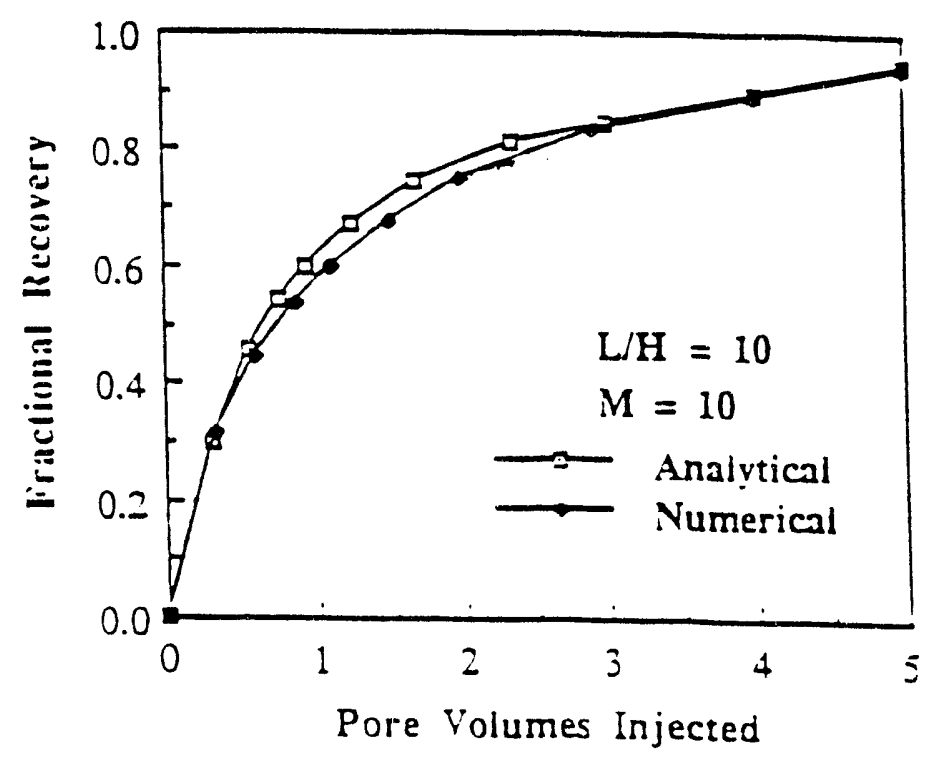

(b)

Fig. 9 Comparison berween the anaivical and numerieal methods at $M=10$.

(a) Efective rilative permeability of displacing tluid

(b) Fracsonai recoveries of resident Iuid 


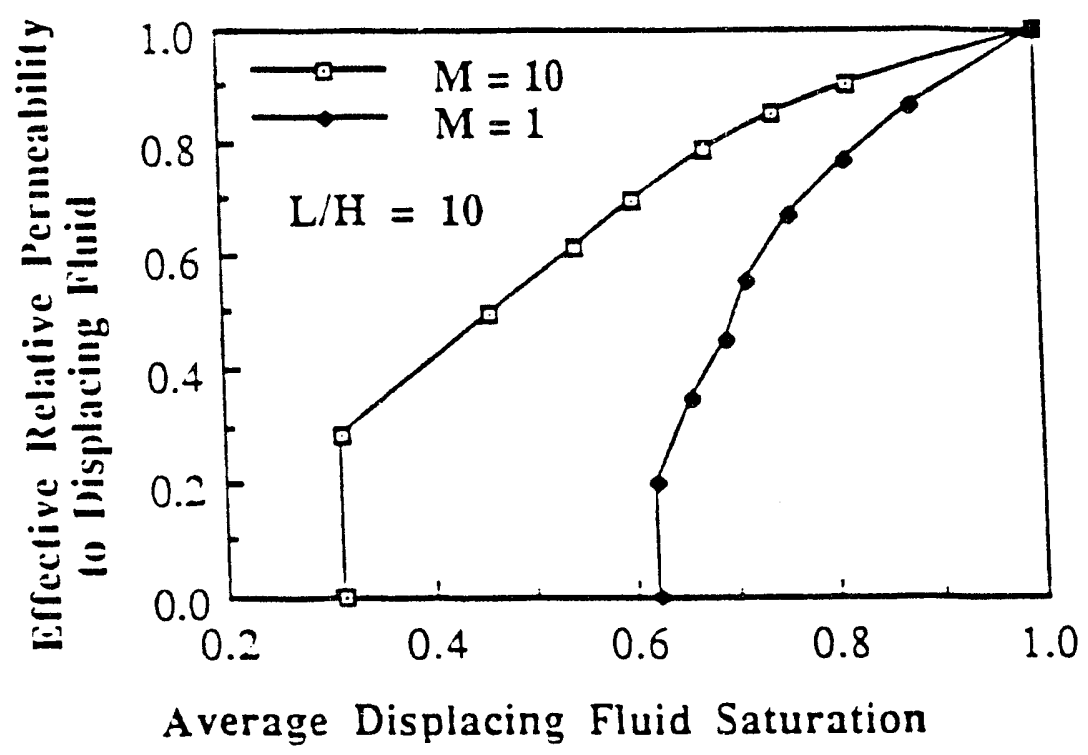

Fig. 10 Comparison of the effective relative permeabilities calculated by the analytical method (VE) for mobility ratios of 1 and 10. 


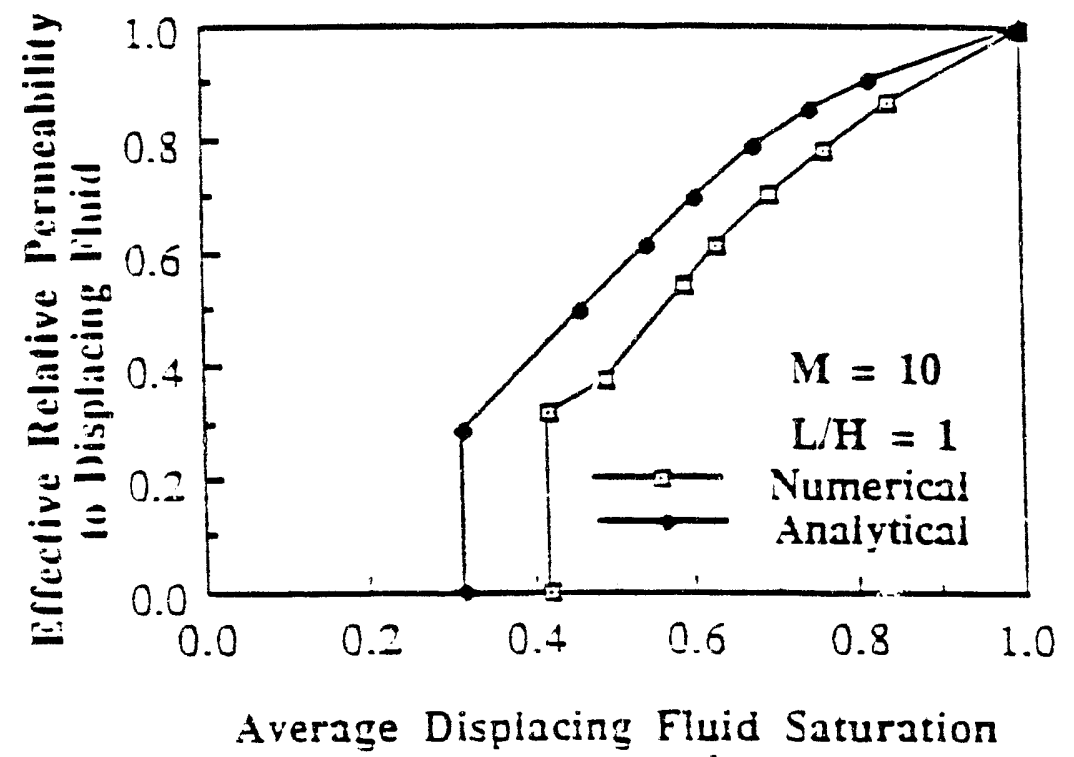

Fi. 11 Comparison of the effectve relarve pe:-enbilities calculated by the analyrical (VE) and numerical methods for an aspece nuto of 1. 


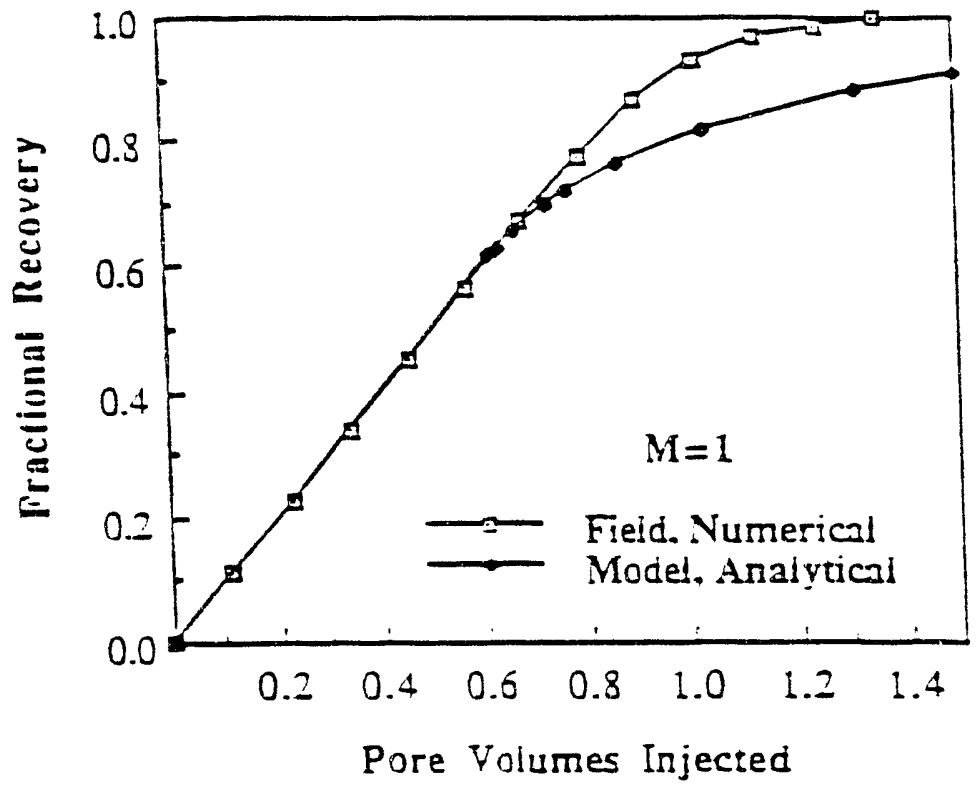

(a)

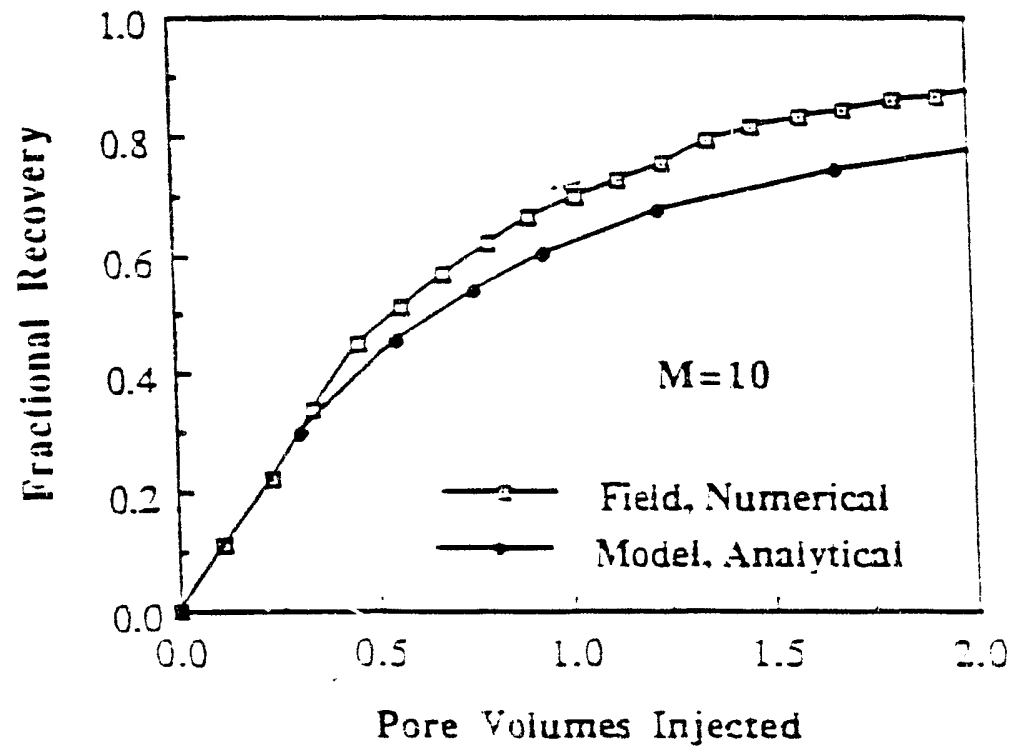

(b)

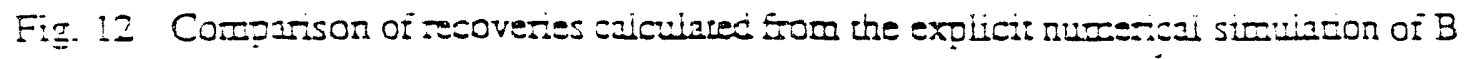
and $C$ unirs and one-di-sessionai coarse Frid simulawons by using effectve

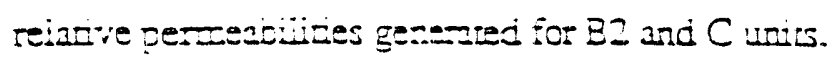
(a) $\mathrm{M}=\mathrm{I}$
(b) $M=10$ 


\section{Subtask 1b - Quantification of Existing Taxonomy through the Use of Correlation, Fractal Representation and Nonlinear Dynamic Statistical Procedures}

We have taken extensive data on the distribution of permeability in the San Andres formation from the North Lawyer Canyon region of the Algerita outcrop in southern New Mexico. We find good agreement between these data and similar data taken from wells in the Wasson field. Current work, through further permeability mapping and X-ray scanning, seeks to define the continuity and geometry of these channels.

\section{Introduction}

Heterogeneity in a permeable medium is the variability of a specific physical parameter (e.g., permeability) with location. Heterogeneous reservoir rock is the result of complex geologic precesses that vary in space and time. The physical and textural variability inherent to geologic media commonly interfere with fluid injection and recovery processes. As such, it is desirable to be able to describe, both qualitatively and quantitatively, any heterogeneities present in the reservoir.

A number of papers have appeared in the literature describing the results of outcrop characterization studies. In several cases, workers have focused on outcrops where they have sampled permeability directly (in-situ) (Chandler, 1986; Goggin et al., 1988b) or indirectly (cores) (Hutchinson et. al., 1987; Jones et al., 1987: Polasek and Hutchinson, 1967; Stalkup, 1986; Tomutsa et al., 1986) in an attempt to detail variability on several scales. By sampling analogous outcrops with depositional formation of interest, much insight into patters of permeability variability in the subsurface can be gained. Moreover, the outcrop affords the opportunity to sample lateral and vertical variability on a scale which resembles inter-well distances found in producing fields, as well as making detailed geologic observations associated with the measured permeability values.

This study focuses on the San Andres formation for several reasons. First, detailed outcrop characterization, including geostatistical analyses, was not previously available for the formation. Second, large, areally extensive outcrops were available, as well as a tremendous amount of subsurface data from the important target horizon in the Permian basin. Any increased understanding of the complex permeability patterns would potentially improve recovery from this horizon by targeting areas for strategically placed infill wells and improving enhanced oil recovery (EOR) process prediction. 


\section{Objectives}

This subtask has the following objectives:

1. Study areal and vertical permeability heterogeneity using San Andres formation outcrops and subsurface core data.

2. Determine representative comelation length(s), if any, for San Andres permeability and porosity.

3. Relate observed permeability characteristics to geologic facies.

4. Cornpare outcrop and subsurface results.

\section{General geology}

The San Andres formation examined in this study lies within the Permian basin. The Guadalupe Mountains, site of the outcrop portion of this study, are generally triangular in outline, with one apex pointing to the south (Fig. 1). Approximately 140 miles (220 $\mathrm{km}$ ) to the northeast lies the Wasson field. The Wasson field is located on the Northwest Shelf and is the location of all subsurface data used in this report (Fig. 1).

The Algerita Escarpment lies along the fault scarp on the east side of Big Dog Canyon, one of two north-south trending valleys which separates the Brokeoff Mountains from the Queen Mesa. The Algerita Escarpment has an average relief of approximately $1400 \mathrm{ft}(430 \mathrm{~m})$ and extends for nearly 15 miles $(24 \mathrm{~km})$. Along the length of the escarpment the work reported on here and that done previously (Hinrichs et al., 1986) has centered on the area known as Lawyer Canyon (formerly Cougar Canyon).

The San Andres formation along the Algerita Escarpment is approximately 1100 to $1200 \mathrm{ft}$ ( 340 to $370 \mathrm{~m}$ ) thick. We will rely on the published stratigraphic section (AE85-1) of Sarg and Lehman (1986) for establishing the general geologic setting of the study area. This particular section passes very near the outcrop study grid, with the study area approximately in units contained in the upper portion of locality $E$ and lower section of locality F (Sarg and Lehman, 1986). The lower portion of the study area contains massive bedded fusulinid lithofacies and an interbedded mud-support dolomite. The upper portion of the study grid contains oolitic grain-supportstones, with prominent cliff-forming, trough crossbedded dolograinstone units (Sarg and Lehman, 1986).

\section{Wasson Field Geology}

The Wasson San Andres field is located on the south eastern edge of the North Basin platform in Gaines and Yoakum counties, Texas. All of the core data used in this 
study came from the Denver Lnit. Yo San Andres core data were examined directly as a part of this study; The description provided below relies on published accounts (Mathis, 1986).

The San Andres formation consists of $1300 \mathrm{ft}(400 \mathrm{~m})$ of dolomite in the Wasson field. The reservoir occurs near the middle of the formation and is overlain by nearly $400 \mathrm{ft}$ $(120 \mathrm{~m})$ of nonporous dolomite. The Wasson reservoir has been subdivided into two portions: a lower interval termed 'Main Pay' (six zones), and an overlying, generally poorer reservoir quality section termed 'First Porosity' (five zones) (Fig. 2). 'Main Pay' is composed of dolomitized open-marine packstones and wackestones. 'First Porosity' is generally shallower-water restricted marine and intertidal rocks. Above 'First Porosity' lies the dense mudstone and anhydrite of the overlying supratidal section which also forms the reservoir seal.

\section{Methodology}

Outcrop

The area chosen for study on the Algerita Escarpment was in an area of good outcrop exposure and relatively good accessibility. The grid was near $(0.5$ miles $[0.8 \mathrm{~km}])$ the previous study area of Hinrichs et al. (1986). The beds sampled in this work can be traced into Hinrichs study area. The final site chosen for data collection was on the north wall of Lawyer Canyon, and is hereafter referred to as the North Lawyer Canyon (NLC) grid (Fig.3).

An area grid was marked on he outcrop. The horizontal base lines of the large grid were aligned parallel to bedding plases while the vertical axes were perpendicular to bedding planes. The grid is approximately $100 \mathrm{ft}(30 \mathrm{~m})$ wide by $80 \mathrm{ft}(24 \mathrm{~m})$ high. Within the large grid A, six small grids B, C, D, E, F, and G were laid out in geologically interesting areas and in places which could be sampled easily.

Sample sites within the study area were positioned at various sampling intervals (densities) using a centric systematic pattern. This pattern forms an efficient means of collecting data useful to spatial characterization (Ripley, 1981). The largest sampling interval was $10 \mathrm{ft}(3.0 \mathrm{~m})$ in the A grid, while data on $1 \mathrm{ft}(0.3 \mathrm{~m})$ and $0.5 \mathrm{ft}(0.15 \mathrm{~m})$ spacing was collected in the smaller girds. Detailed 1 in $(0.025 \mathrm{~m}$ ) (B and E grids) and 0.5 in $(0.013 \mathrm{~m}$ ) (B grid) data was also collected. Data in the vertical (VT) transect was sampled every 2 in $(0.051 \mathrm{~m})$.

We prepared the individual sample sites prior to measurement with the permeameter, since the device requires a relatively flat surface. Also, surface preparation 
removed the outer weathering surface present on the outcrop. Each sample site was ground using a grinder. The resulting sample site was generally flat and was about 3-5 in 2 (0.0)2$0.003 \mathrm{~m}^{2}$ ) in area. The grinding did affect the permeameter measurement, however (see below).

Permeability was measured at each of the individual sample sites using an experimental mechanical field permeameter (MFP). The MFP is a simple gas flow measuring device which is capable of making many rapid, non-destructive permeability measurements in a field setting. For complete details of the construction of the MFP and numerical characterization of the flow response, see Goggin et al. (1988a).

To complement the field-derived MFP permeabilities and to provide geologic characterization, we acquired core plugs at various locations within the study area. The core plugs were obtained using a portable, gasoline-driven, water-cooled core plug device. Samples were taken as near as possible to the location of the MFP measuring point in an attempt to provide data that would be comparable. Table 1 shows a complete summary of the data collected.

\section{Subsurface}

The data from the Wasson field consist of core samples taken from ten closely spaced wells. Data from both whole core and core plugs were available from lab reports held in company files. Whole core data included two, and in two cases, three permeability measurements, and porosity. Two permeabilities $\left(\mathrm{k}_{1}, \mathrm{k}_{2}\right)$ were measured in the horizontal plane orthogonal to each other and the third permeability, where present, was a vertical permeability $\left(\mathrm{k}_{\mathrm{v}}\right)$. Whole core permeability was measured in a Hassler sleeve and porosity was obtained by Boyle's Law. Core plug data was also available for some wells, and consisted of permeability (k) and porosity $(\phi)$. Table 2 summarizes the available subsurface data.

\section{Laboratory Analyses}

Core plugs from the NLC grid area were analyzed for permeability using the MFP as well as conventional 1-D (Hassler sleeve) measurements. MFP-derived permeability estimates were made at the end of each core plug, resulting in, for most samples, two permeability values. The purpose of the measurement procedure was to detect any end-toend permeability variability within the sample. Hassler sleeve measurements were made with a confining nitrogen pressure between $225-250 \mathrm{psig}(1550 / 1720 \mathrm{kPa})$. In addition to 
permeability, standard Boyle's Law-type porosity techniques were used on the core plugs to measure porosity.

Thin sections were prepared from each core plug sample using the ends trimmed during sample preparation.

\section{Quantitative Characterization}

Population Statistics - All of the data collected were analyzed initially using basic population statistics. Standard values calculated included arithmetic mean, variance, and coefficient of variation $\left(C_{v}\right)$. In addition, each data set was considered together and analyzed for distribution type. The three distributions considered were the normal, lognormal, and power normal (the p-normal of Jensen et al., 1987). The appropriate p-exponent was estimated for each data set as described in Kittrige (1988). The estimated $p$ is given the symbol $\hat{\mathrm{p}}$ or PHAT in the computer generated plots.

Geostatistics - Geostatistics differs from classical probability-based statistics in that it is specifically designed to process data which may exhibit varying degrees of spatial correlation. The primary tool used here was the variogram, which is a mathematical function used to quantify the degree of spatial variability or continuity for a variable defined over a continuum. We chose the variogram over the corelogram because it is unbiased (Yang and Lake, 1988-89). The variogram (or semi-variogram) was estimated using the following relation:

$$
\gamma^{*}(h)=\frac{1}{2 N(h)} \sum_{i=1}^{N(h)}\left\{Z\left(x_{i}\right)-Z\left(x_{i}+h\right)\right\}^{2}
$$

A modified form of the above equation was used to calculate a directional variogram:

$$
\gamma^{*}\left(H_{K}, \theta \pm \Delta \theta\right)=\frac{\sum_{j=1}^{N\left(H_{K}\right)}\left\{h_{j}\left(Z\left(x_{i}\right)-Z\left(x_{i}+h_{j}\right)\right)^{2}\right\}}{2 \sum_{j=1}^{N\left(H_{K}\right)} h_{j}}
$$


By analyzing results in different directions, spatial anisotropy in the parameter being considered might be recognized. The spherical model was used in modeling all the variograms in this work:

$$
\gamma^{*}(h)=C_{O}+C\left[\frac{3 h}{2 a}-\frac{h^{3}}{2 a^{3}}\right]
$$

In Eq. (3), a is the variogram range, $C_{o}$ is the nugget, and $C+C_{o}$ is the sill. The range is the distance over which correlation exists, $C_{0}$ is variance smaller than the sample spacing and $\mathrm{C}+\mathrm{C}_{\mathrm{o}}$ is the sample variance.

\section{Results}

\section{Population Statistics}

Outcrop - The NLC permeability data were analyzed for statistical properties and distribution type by grid and by region within the grid. Three data regional grids were formed: all NLC data, lower NLC data (grids E, F, VT-1, VT-2, and lowest 4 rows of grid A) and upper NLC data (grids B, C, D, G, VT-3, and upper 4 rows of grid A). These groupings were shown to conform to the geologic classification shown on Fig. 4. Table 3 shows results of the statistical analyses.

Permeability data for the three regional grids is approximately log-normally distributed $(-0.062<\hat{\mathrm{p}}<0.029)$, while the individual grids show more variation in distribution type $(-1.41 \mathrm{n}<\hat{\mathrm{p}}<0.284)$. Data taken over several geologic types show large coefficients of variation $C_{v}$, while a small $C_{v}$ occurs in the more geologically confined data sets. The upper regional grid, consisting primarily of packstones, is significantly larger in permeability than the primarily wackestone lower regional grid.

Subsurface - The core data were analyzed by permeability type, with similar types from all the cores combined into a single data set. The results of the statistical analyses are shown in Table 4. Permeability data are essentially log-normally distributed, with $\hat{p}$ values all greater than 0 but less than 0.1 . The core plug permeability data had a much larger variance and $C_{v}$ values than any of the whole core data, reflecting the greater averaging effect of the whole core samples. The two porosity data sets showed power-normal distributions which were non-normal, in contrast to the commonly assumed normal distribution. The comparison between NLC and SWAU data is complicated by the difference in support of averaging volume. Probably the most meaningful comparison is between the NLC minipermeameter data on all of the grids ("All data" in Table 3) and the 
core plug data in Table 4 , since the latter were undifferentiated with respect to geologic classification. The NLC data has a significantly larger mean permeability than the WSAU data but the two data sets agree quite well in terms of distribution type and coefficient of variation. This agreement in statistical properties between outcrop and subsurface permeability data, except for the mean, has also been observed by Goggin et al. (1989) in eolian sand deposits. The difference in mean permeability has a number of causes, but most likely the reduction caused by overburden pressure is the most significant.

\section{Areal and Vertical Pattems}

The permeability and subsurface porosity data were studied using contour plots (outcrop grids) and profiles (vertical transect and core data) to obtain a visual representation of the spatial variability. Figure 5a shows a representative case from Grid B. Low permeability occurs in an area corresponding to a mudstone bed. Elsewhere, the permeability typically occurs as isolated pods of large permeability surrounded by generally low permeability matrix rock. A similar pattern occurs on a very detailed scale (Fig. 5b), where some of the large values align approximately with the measured cross-bed direction.

The vertical transect and core data exhibited rapid variation in permeability and porosity over a short vertical interval. Grainstone data had distinctly larger permeability while the mudstone data showed a sharp break to lower permeability values (Fig. 6a). Subsurface core data showed similar variability to that observed in the outcrop (Fig. 6b) but with a reduced mean.

\section{Variogram Analysis}

All variogram analyses were conducted on appropriately transformed data: data sets with $-0.1<\hat{\mathrm{p}}<0.1$ used a $\log _{10}$ transform, while the $\hat{\mathrm{p}}$ value was used with all other data sets. Tables 5 (outcrop) and 6 (subsurface) give results of the variogram modeling. We concentrate on results from Grid B and the vertical transect in the outcrop and a single well in the subsurface. See Kittridge for variograms from each outcrop grid and all cores.

The $1 \mathrm{ft}(0.3 \mathrm{~m})$ data in Grid B exhibited a pure nugget effect (no spatial correlation), which was likely because of the small number of data. Spatial correlation was detected in the $0.5 \mathrm{ft}(0.15 \mathrm{~m})$ data with a range of $1-2 \mathrm{ft}(0.6-0.9 \mathrm{~m})$ (Fig. $7 \mathrm{a})$. In the detail area of Grid $B$, the 1.0 in $(0.026 \mathrm{~m})$ data showed a range of $0.25-0.3 \mathrm{ft})(0.076-0.09$ $\mathrm{m}$ ). The extremely detailed 0.5 in $(0.013 \mathrm{~m}$ ) data revealed a similar predicted range (Fig. $7 b)$ which was very similar in each direction considered. suggesting that the autocorrelation in the permeability data is sparially isotropic within individual bedded units. 
The outcrop vertical transect data was analyzed as 1-D data, as was all subsurface permeability and porosity data from the Wasson field. The predicted range for the uppermost outcrop transect segment, which is geologically similar to the cored interval in the Wasson field, showed a range of approximately $11 \mathrm{ft}(3.4 \mathrm{~m})$ (Fig. 8a). The sharp drop in $\gamma^{*}$ near $h=15 \mathrm{ft}(4.6 \mathrm{~m})$ suggests the presence of a whole-effect model in the vertical direction. Similarly, the average predicted correlation range for $k_{1}, k_{2}$ and $k_{v}$ was 11.2 (Fig. 8b), 13.0, and $9.5 \mathrm{ft}(3.4,4.0$, and $2.9 \mathrm{~m})$, respectrvely. Porosity data indicated a vertical range of $10.7 \mathrm{ft}(3.3 \mathrm{~m})$ in good agreement with th.e permeability data.

\section{Accuracy of MFP Permeability Measurements}

A persistent question regarding the outcrop data collection procedure concerns the impact of grinding (sample site preparation) on the MFP measurements of permeability. Using hand samples collected from the field area, MFP measurements were made on an unaltered surface (weathered) and on a fresh, broken surface, once underground and then following grinding. Every attempt was made to duplicate the grinding as it was performed in the outcrop.

Figure 9 shows the results of this experiment in histogram form. In general, permeability is reduced, on each surface, following grinding. Assessing the cause of the permeability decrease is conjectural, but it is likely that the grinding process is driving generated fines into the pore space and reducing permeability. Fines migration and plugging of pore space is much more significant in the low permeability samples.

The experiments conducted confirm only that the grinding process reduces the permeability measured by the MFP on the weathered and broken surfaces. These results do not allow any conclusions to be made regarding the relationship between permeability measured on ground surface and the absolute or true matrix permeability as measured on a core plug. Nevertheless, this reduction in minipermeameter values constitutes a major qualification of this work inasmuch as it will augment the already significant differences between the mean permeability in outcrop and subsurface. We feel that the deviation does not affect conclusions regarding the nature of the permeability distribution and how it relates to geology, nor does it apparently affect the similarity in distribution type and coefficient of variation between the outcrop and subsurface.

Figure 10 provides a direct comparison of MFP-measured permeability and Hassler sleeve permeability. At moderate to large permeabilities (5-5000 md [0.005-5 $\left.\mu \mathrm{m}^{2}\right]$ ), the MFP-derived values agree reasonably well with the Hassler sleeve permeability. A substantial deviation away from the unit-slope line occurs below Hassler sleeve values of 
$10 \mathrm{md}\left(0.01 \mu \mathrm{m}^{2}\right)$. This departure is caused by the lower limit of detection and resolution of the MFP, which is estimated to be approximately $0.5 \mathrm{md}\left(0.0005 \mu \mathrm{m}^{2}\right)$, but may in fact be in the range of $2-5 \mathrm{md}\left(0.002-0.005 \mu \mathrm{m}^{2}\right)$, as indicated by Fig 10. The sandstone data of Goggin et al. (1988a) exhibited agreement down to $1 \mathrm{md}\left(0.001 \mu \mathrm{m}^{2}\right)$.

Subsurface and Outcrop Agreement

Table 7 summarizes comparisons made between outcrop and subsurface data and results. The geologic units are of the same formation and are known to be contiguous between the outcrop and the Wasson field. Rock fabrics and pore types observed in the NLC outcrop samples are similar to those reported for the Wasson field, in spite of the difference in faunal content between the two locations.

Mean permeability values are significantly larger in the outcrop than in the subsurface. Predicted variogram range for the outcrop vertical transect permeability data compares very well with the average value determined for the subsurface data. All data and results support the hypothesis that the San Andres present on the Algerita Escarpment is a valid analogue to the subsurface San Andres of the Northwestern shelf area. Geostatistical results derived from outcrop data should be useful in modeling the subsurface San Andres.

\section{Conclusions}

We have studied a portion of a San Andres outcrop using a field minipermeameter. Based on the work reported here, we offer the following conclusions:

1. Outcrop permeability data varied widely, from a low of less than $0.1 \mathrm{md}$ $\left(\sim 0.0001 \mu \mathrm{m}^{2}\right)$ to over $2000 \mathrm{md}\left(2 \mu \mathrm{m}^{2}\right)$, a range of 4 factors of ten.

2. The permeability measured by the minipermeameter was lower than true matrix permeability because of the grinding procedure used during sample site preparation.

3. Permeability variability in outcrop was not confined to bed-to-bed changes; rather, substantial variability occurred within individual bedded units.

4. Mean permeability agrees poorly between subsurface and outcrop. Other statistical measures, such as distribution type, coefficient of variation and variogram range, show much better agreement.

5. Outcrop permeability data exhibited no marked permeability anisotropy in the predicted spatial correlation length on the scale of the individual bedded units studied. 
6. We observed two distinct scales of spatial correlation, depending on the spacing of the data used. Predicted correlation lengths were $3.5 \mathrm{ft}(0.9-1.5 \mathrm{~m})$ for the $1 \mathrm{ft}(0.3$ $\mathrm{m})$ and $0.5 \mathrm{ft}(0.15 \mathrm{~m})$ data spacing, and $0.25 \mathrm{ft}(0.076 \mathrm{~m})$ for the 1 in $(0.025 \mathrm{~m}){ }^{\text {. }}$ and 0.5 in $(0.013 \mathrm{~m})$ data.

7. The Algerita Escarpment appears to be a good analogue for the producing interval in the Wasson field.

\section{Beferences}

Chandler, M.A., "Depositional Controls on Permeability in an Eolian Sandstone Sequence, Page Sandstone, North Arizona," M.A. Thesis, The University of Texas at Austin, Texas (1986)

Goggin, D.J., R.L. Thrasher, and L.W. Lake, "A Theoretical and Experimental Analysis of Minipermeameter Response Including Gas Slippage and High Velocity Flow Effects," In Situ, vol 12 (1 and 2), 1988, 79-116.

Goggin, D.J., M.A. Chandler, G. A. Kocurek and L.W. Lake, "Patterns of Permeability in Eolian Deposits," SPE Formation Evaluation (June 1988) 297-306.

Goggin, D.J., M.A. Chandler, G. A. Kocurek and L.W. Lake, "Permeability Transects in Eolian Sands and Their Use in Generating Random Permeability Fields," SPE Paper 19586 to be presented at the 1989 SPE Annual Technical Conference and Exhibition, San Antonio, Oct. 8-11.

Hinrichs, P.D., F.J. Lucia and R.L. Mathis, "Permeability Distribution and Reservoir Continuity in Permian San Andres Shelf Carbonates, Guadalupe Mountains, New Mexico," Proc. Hydrocarbon Reservoir Studies - San Andres/Greyburg Formations, Permian Basin Research Conference (PBS-SEPM Pub. No. 86-26), Midland, Texas (1986), 31-36.

Hutchinson, C.A., Jr., C.F. Dodge and T.L. Polasek, "Identification, Classification, and Prediction of Reservoir Nonuniformities Affecting Production Operations," Journal of Petroleum Technology (March 1987) 223-229.

Jensen, J.L., D.V. Hinkley, and L.W. Lake, "A Statistical Study of Reservoir Permeability: Distributions, Correlations, and Averages," SPE Formation Evaluation (Dec. 1987), 461-468. 
Jones, J.R., Jr., A.J. Scott and L.W. Lake, "Reservoir Characterization for Numerical Simulation of Mesaverde Meanderbelt Sandstones, Northwestern Colorado," SPE Formation Evaluation (March 1987) 97-107.

Kittridge, M.G., "Analysis of Areal Permeability Variations - San Andres Formation (Guadalupian): Algerita Escarpment, Otero County, New Mexico," M.S. Thesis, The University of Texas at Austin, 1988.

Mathis, R.L., "Reservoir Geology of the Denver Unit - Wasson Sand Andres Field, Gaines and Yoakum Counties, Texas," Proc. Hydrocarbon Reservoir Studies - San Andres/Grayburg Formations, Permian Basin Research Conference (PBS-SEPM Pub. No. 86-26), Midland, Texas (1986), 43-48.

Polasek, T.L., and C.A. Hutchinson, Jr., "Characterization of Non-Uniformities Within a Sandstone Reservoir from a Fluid Mechanics Standpoint," Proc. Seventh World Petroleum Congress, (1967) 397-407.

Ripley, B.D., Spatial Statistics, John Wiley and Sons, New York (1981), 19-22.

Sarg, J.F. and P.J. Lehmann, "Facies and Stratigraphy of Lower-Upper San Andres Shelf Crest and Outer Shelf and Lower Grayburg Inner Shelf," Proc. San Andres/Grayburg Formations, Guadalupe Mountains, New Mexico and Texas, Permian Basin Research Conference (PBS-SEPM Pub. No. 86-25), Midland, Texas (1986), 9-36.

Stalkup, F.I., "Permeability Variations Observed at the Faces of Crossbedded Standstone Outcrops," in Reservoir Characterization, L.W. Lake and HB. Carroll (eds.), Academic Press, Orlando, FL, 1986.

Tomutsa, L, S.R. Jackson and M. Szpakiewicz, "Geostatistical Characterization and Comparison of Outcrop and Subsurface Facies: Shannon Shelf Sand Ridges," SPE paper 15127 presented at the 1986 California Regional Meeting of the SPE, Oakland, April 2-4

Yang, An-Ping and L.W. Lake, "The Accuracy of Autocorrelation Estimates," In Situ, vol. 12 (4), 1988-89, 275-317.

\section{Nomenclature}

a Range in theoretical variogram model, $[=] \mathrm{L}$

$\mathrm{C}_{0} \quad$ Nugget in theoretical variogram model

$\mathrm{CV} \quad$ Coefficient of variation, dimensionless 


\begin{tabular}{|c|c|}
\hline $\mathrm{h}$ & Data spacing, $[=] \mathrm{L}$ \\
\hline $\mathrm{H}_{\mathrm{K}}$ & Class size, $[=] \mathrm{L}$ \\
\hline k & Minipermeameter (air) or core plug permeability, $l=j L^{2}$ \\
\hline $\mathrm{k}_{1}, \mathrm{k}_{2}$ & Whole core permeability perpendicular to $\mathrm{k} 1-\mathrm{k} 2$ plane, $l=\mathrm{l}^{2}$ \\
\hline iv & Number of data points \\
\hline$\hat{\mathrm{p}}$ & Estimated transform exponent \\
\hline Z & Random variable value \\
\hline \multicolumn{2}{|c|}{ Greek } \\
\hline & Variogram \\
\hline & Direction for variogram calculation \\
\hline
\end{tabular}

\section{List of Tables}

$\begin{array}{cl}\text { Table } & \\ 1 & \text { Summary of NLC Data Set } \\ 2 & \text { Summary of Wasson Field Data Set } \\ 3 & \text { NLC Statistical Summary } \\ 4 & \text { Wasson Field Statistical Summary } \\ 5 & \text { NLC Variogram Results } \\ 6 & \text { Wasson Field Variogram Results } \\ 7 & \text { Comparison of Results: Outcrop - Subsurface }\end{array}$

\section{List of Figures}

Figure

1 Location of Algerita escarpment and Wasson fields in Permian Basin (after Hinrichs et al., 1986)

2 Wasson field San Andres type log (after Mathis, 1986)

3 Location of NCL study grid on Algerita escarpment

4 Grid locations and gerieral geology of NLC study area

5 Permeability contour plots 
6 Permeability profiles

(a) NLC vertical transect

(b) Wasson field Well 1

7 Calculated variograms
(a) NLC Grid B (0.5 ft data)
(b) NLC Grid B (0.5 in data)

8 Calculated variograms

(a) NLC vertical transect ( 2 in data)

(b) Wasson field Well 1 ( $\mathrm{K} 1,1 \mathrm{ft}$ data)

9 Permeability reduction due to grinding process, weathered and broken surfaces

10 Comparison of MFP and absolute permeabilities, NLC core plugs 
TABLE 1

Summary of NLC Data Set

\begin{tabular}{cccc}
\hline $\begin{array}{c}\text { Grid } \\
\text { Designation }\end{array}$ & $\begin{array}{c}\text { Sample } \\
\text { spacing }\end{array}$ & $\begin{array}{c}\text { Number } \\
\text { of data }\end{array}$ & $\begin{array}{c}\text { Number of } \\
\text { core plugs }\end{array}$ \\
\hline A & $10.0 \mathrm{ft}$ & 72 & 22 \\
B & $1.0 \mathrm{ft}$ & 36 & 8 \\
& $0.5 \mathrm{ft}$ & 121 & - \\
& $1.0 \mathrm{in}$ & 128 & - \\
C & $0.5 \mathrm{in}$. & 576 & 13 \\
D & $1.0 \mathrm{ft}$ & 87 & 10 \\
E & $1.0 \mathrm{ft}$ & 132 & 5 \\
F & $1.0 \mathrm{ft}$ & 68 & 2 \\
G & $1.0 \mathrm{ft}$ & 42 & 7 \\
VT & $1.0 \mathrm{ft}$ & 36 & -
\end{tabular}


TAELE 2

Summary of Wasson Field Data Set

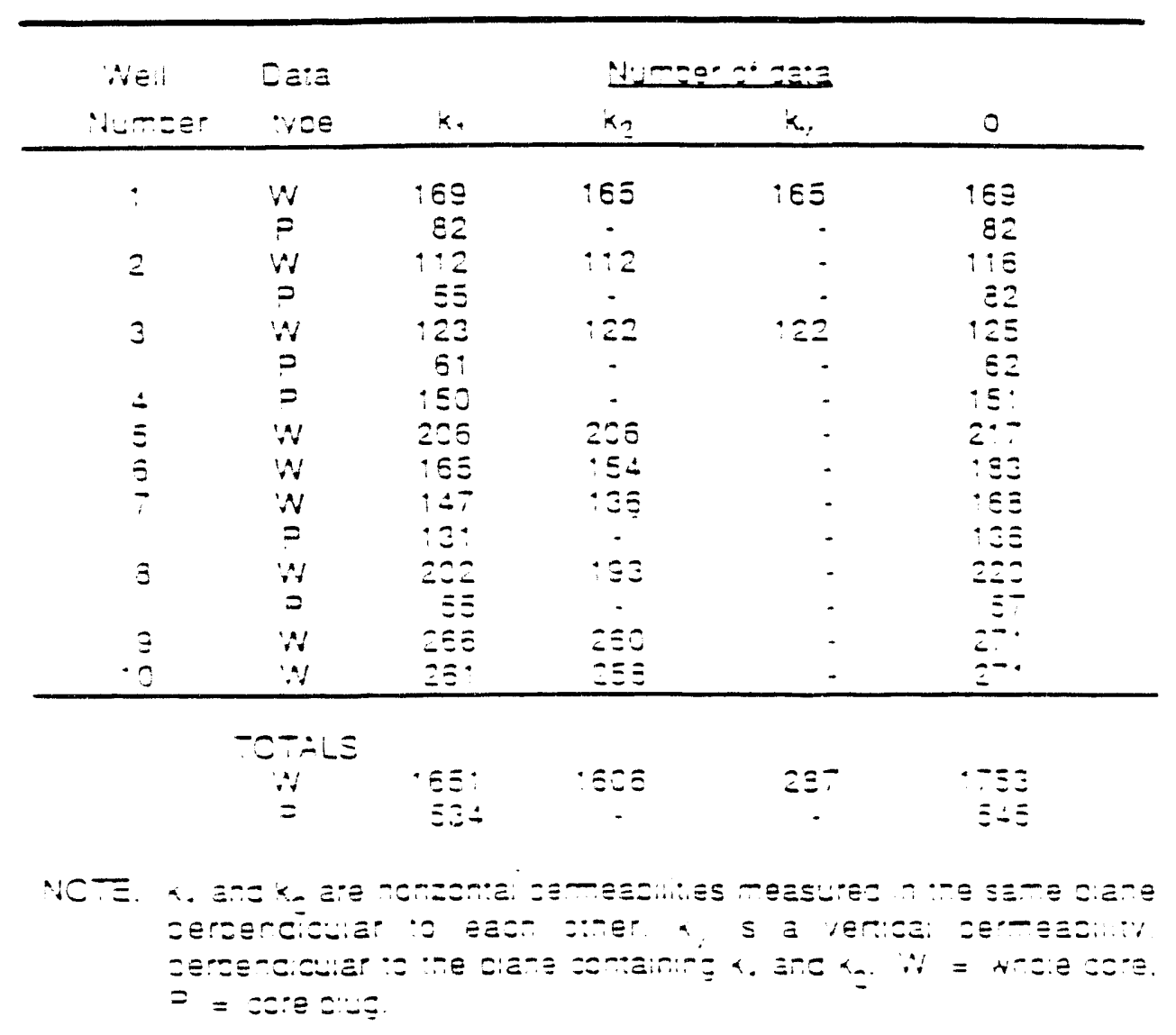




\section{TABLE 3}

NLC Statistical Summary

\begin{tabular}{|c|c|c|c|c|c|c|}
\hline Designation & $\begin{array}{l}\text { Sample } \\
\text { Spacing }\end{array}$ & $\mathrm{N}$ & $\begin{array}{l}\text { MEAN } \\
\text { md }\end{array}$ & $\begin{array}{c}\text { VARIANCE } \\
m d^{2}\end{array}$ & $\hat{p}$ & $C_{v}$ \\
\hline All data & various & 1605 & 44.2 & 21000 . & -.062 & 3.27 \\
\hline Lower grid & various & 410 & 3.04 & 22.9 & .029 & 1.58 \\
\hline Upper grid & various & 1195 & 58.4 & 27400 . & -.012 & 2.83 \\
\hline A & $10 \mathrm{ft}$ & 72 & 34.7 & 11600. & -.300 & 3.11 \\
\hline B & $\begin{array}{c}1 \mathrm{ft} \\
0.5 \mathrm{ft} \\
1 \mathrm{in} \\
0.5 \mathrm{in}\end{array}$ & $\begin{array}{c}36 \\
115 \\
128 \\
586\end{array}$ & $\begin{array}{l}22.4 \\
22.2 \\
55.9 \\
48.7\end{array}$ & $\begin{array}{l}1690 . \\
1820 \\
3770 . \\
2440\end{array}$ & $\begin{array}{r}-.050 \\
-.154 \\
.056 \\
.284\end{array}$ & $\begin{array}{l}1.83 \\
1.92 \\
1.10 \\
1.01\end{array}$ \\
\hline C & $1 \mathrm{ft}$ & 87 & 155.2 & 56000. & -.136 & 1.53 \\
\hline D & $1 \mathrm{ft}$ & 132 & 136.1 & 169000 & -.270 & 3.02 \\
\hline$E$ & $\begin{array}{l}1 \mathrm{ft} \\
1 \mathrm{in}\end{array}$ & $\begin{array}{c}68 \\
141\end{array}$ & $\begin{array}{l}2.73 \\
1.36\end{array}$ & $\begin{array}{l}14.6 \\
3.77\end{array}$ & $\begin{array}{r}-.154 \\
.024\end{array}$ & $\begin{array}{l}1.40 \\
1.43\end{array}$ \\
\hline $\mathrm{F}$ & $1 \mathrm{ft}$ & 42 & 3.57 & 13.4 & -1.41 & 1.02 \\
\hline G & $\begin{array}{c}1 \mathrm{ft} \\
0.5 \mathrm{ft}\end{array}$ & $\begin{array}{c}36 \\
121\end{array}$ & $\begin{array}{l}3.38 \\
5.04\end{array}$ & $\begin{array}{l}17.4 \\
53.1\end{array}$ & $\begin{array}{l}-.360 \\
-.468\end{array}$ & $\begin{array}{l}1.23 \\
1.45\end{array}$ \\
\hline $\begin{array}{l}V T \\
V T-1 \\
V T-2 \\
V T-3\end{array}$ & $\begin{array}{l}2 \text { in } \\
2 \text { in } \\
2 \text { in } \\
2 \text { in }\end{array}$ & $\begin{array}{c}241 \\
51 \\
65 \\
125\end{array}$ & $\begin{array}{l}20.0 \\
4.30 \\
5.65 \\
33.9\end{array}$ & $\begin{array}{r}4960 . \\
43.3 \\
54.9 \\
9160 .\end{array}$ & $\begin{array}{r}-.225 \\
-.287 \\
.022 \\
-.234\end{array}$ & $\begin{array}{l}3.52 \\
1.53 \\
1.31 \\
2.82\end{array}$ \\
\hline
\end{tabular}

$V T$ is vertical transect; all other data are grids. VT is the combination of VT-1 through VT-3. 


\section{TABLE 4}

Wasson Field Statistical Summary

\begin{tabular}{ccccccc}
\hline Variable(s) & Type & $\mathrm{N}$ & $\begin{array}{c}\text { MEAN } \\
\mathrm{md}\end{array}$ & $\begin{array}{c}\text { VARIANCE } \\
\mathrm{md}^{2}\end{array}$ & $\hat{\mathrm{p}}$ & $\mathrm{C}_{\mathrm{V}}$ \\
\hline All & $\mathrm{W}$ & 3544 & 12.2 & 360. & .093 & 1.56 \\
$k_{1}$ & $\mathrm{~W}$ & 1651 & 13.8 & 410. & .098 & 1.47 \\
$k_{2}$ & $\mathrm{~W}$ & 1606 & 11.2 & 314. & .089 & 1.59 \\
$k_{v}$ & $\mathrm{~W}$ & 287 & 8.68 & 295. & .017 & 1.98 \\
$k$ & $\mathrm{P}$ & 534 & 15.5 & 2500. & .023 & 3.23 \\
$f$ & $\mathrm{~W}$ & 1753 & 11.6 & 15.6 & .570 & 0.342 \\
$f$ & $\mathrm{P}$ & 545 & 11.6 & 19.9 & .807 & 0.385
\end{tabular}

NOTE: $k_{1}$ and $k_{2}$ are horizontal permeabilities measured in the same plane, perpendicular to each other. $k_{v}$ is a vertical permeability, perpendicular to the plane containing $k_{1}$ and $k_{2} . W=$ whole core, $P=$ core plug. 


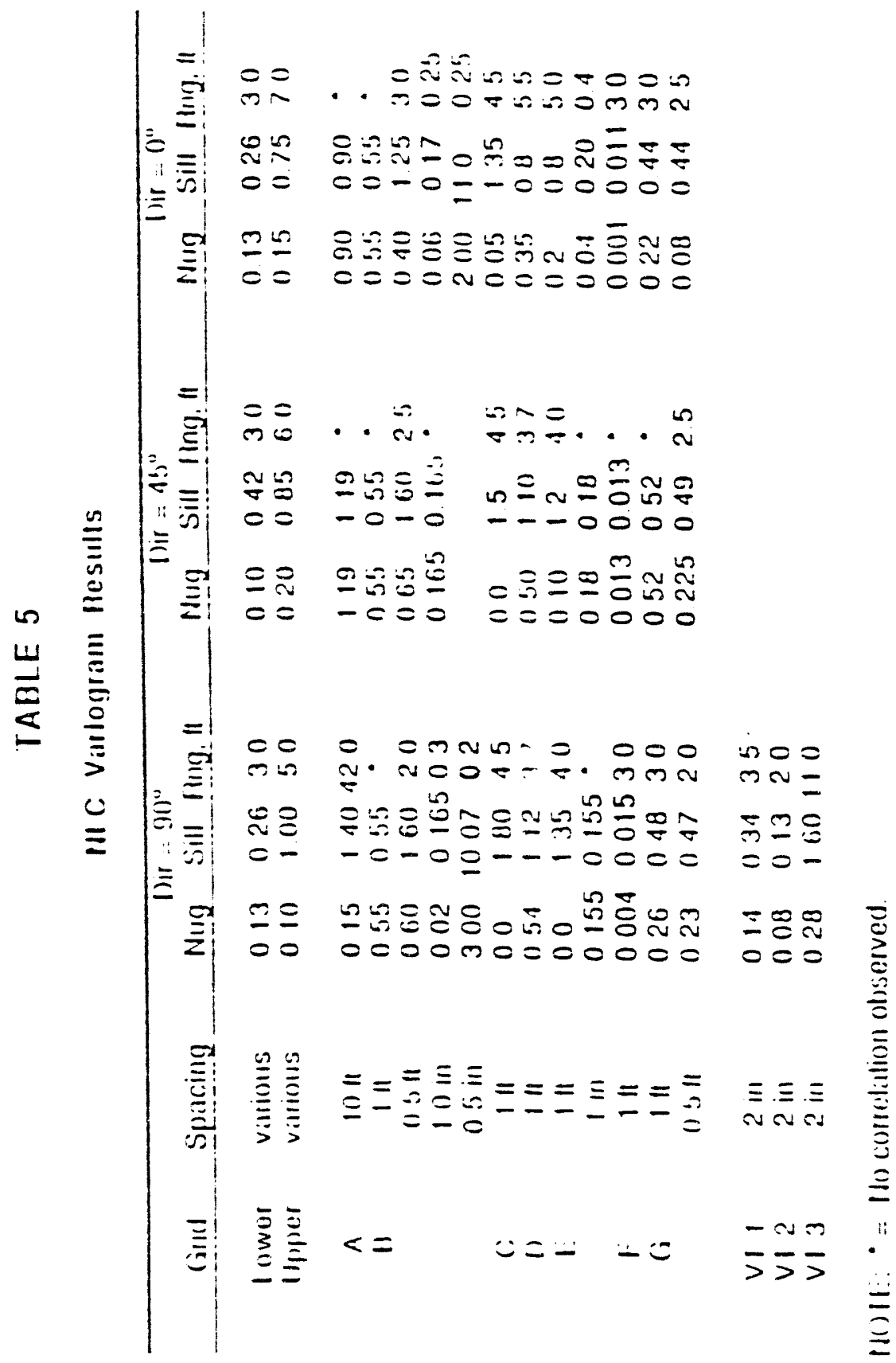




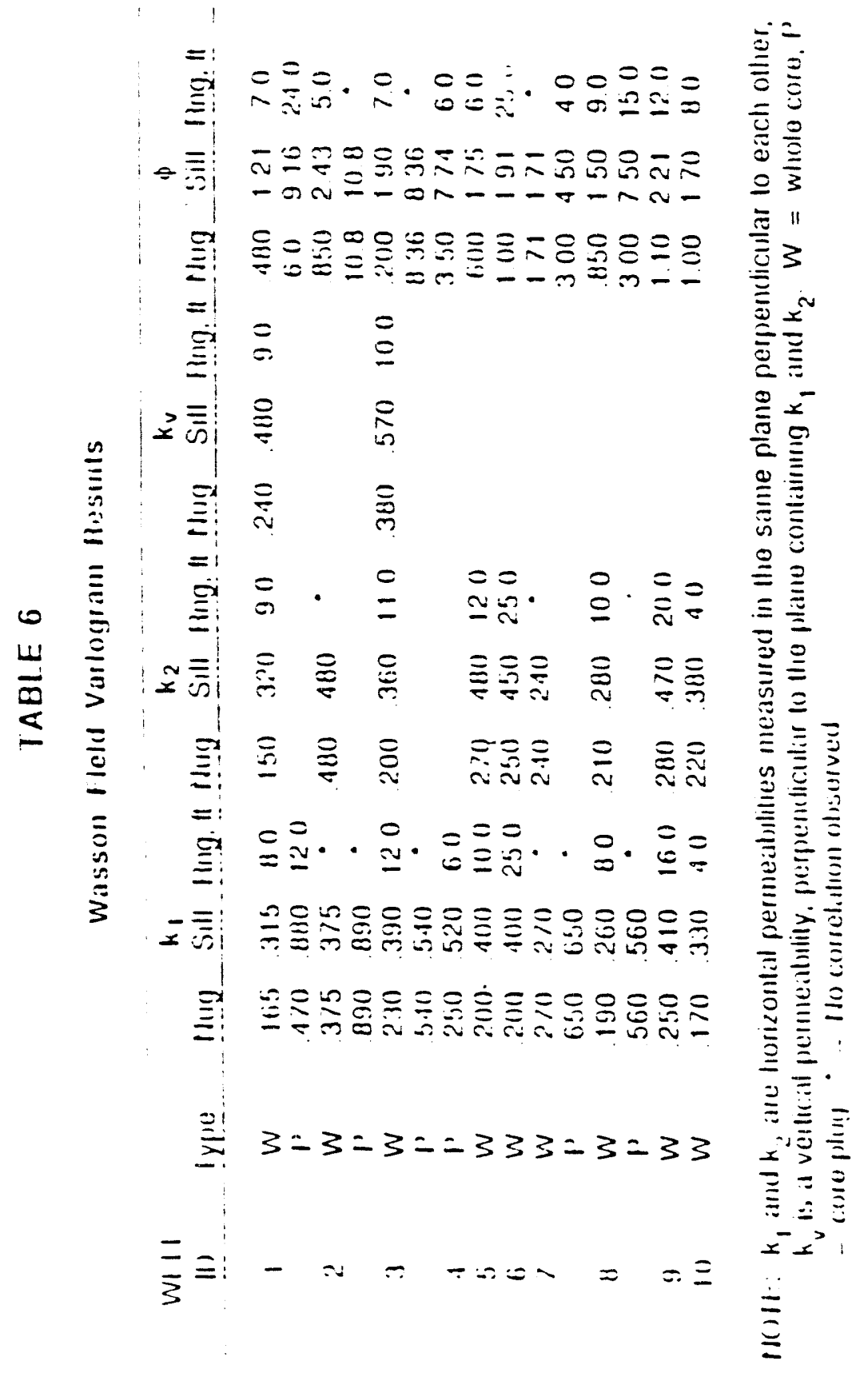




\section{TABLE 7}

\section{Comparison of Results: Outcrop - Subsurface}

\begin{tabular}{|c|c|c|c|}
\hline liem & Uuicico & Sucsunace & Comitieni \\
\hline Fock fadric & $\begin{array}{l}\text { muast.wksi } \\
\text { pkst.grnst }\end{array}$ & wkst, pkst & similar \\
\hline Pare (ypels) & BP.WP.MO & $\begin{array}{l}\text { EC.VUG.MO } \\
\text { WC.NP }\end{array}$ & similar \\
\hline Faunal content & tusuilinids & oric & $\begin{array}{l}\text { difference in } \\
\text { ginal deoositional } \\
\text { location }\end{array}$ \\
\hline Mean k, mc & 442 & 12.2 & $\begin{array}{c}\text { overourcen in } \\
\text { oulcros }\end{array}$ \\
\hline Mlean o. \% & 10.9 & 11.6 & similar \\
\hline $\begin{array}{l}\text { kisiribution } \\
\text { iype }\end{array}$ & $\begin{array}{l}\text { log-normal. } \\
\text { power normal }\end{array}$ & loğ-ricrmal & $\begin{array}{l}12 \hat{o} \text { values < } 0 \\
\text { in outcrso daia }\end{array}$ \\
\hline o cisinicution & \multicolumn{3}{|c|}{$\begin{array}{l}\text { power normal } \\
(\hat{p}=.57, .81)\end{array}$} \\
\hline$C_{1}(\operatorname{tor} k)$ & $1.01-3.02$ & $1.47-3.23$ & similar \\
\hline Range. & $\begin{array}{l}\text { varies wiln samiole } \\
\text { soacing: } 40.3 .25\end{array}$ & $\begin{array}{c}\text { avg. } \text { venical }=9.5 \\
\text { :c : } 0\end{array}$ & $\begin{array}{c}\text { outcico verical } \\
\text { iransec: } \\
\text { maicres sucsurace }\end{array}$ \\
\hline 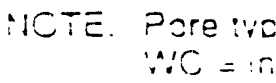 & $\begin{array}{l}\text { es - } 2 C=\text { nterchisi } \\
\text { :acrysianine } \because P=\end{array}$ & $\begin{array}{l}\text { he. VUG = vugg: } \\
\text { :izaar:c:o. }\end{array}$ & Y. $: M O=$ mo:c: \\
\hline
\end{tabular}




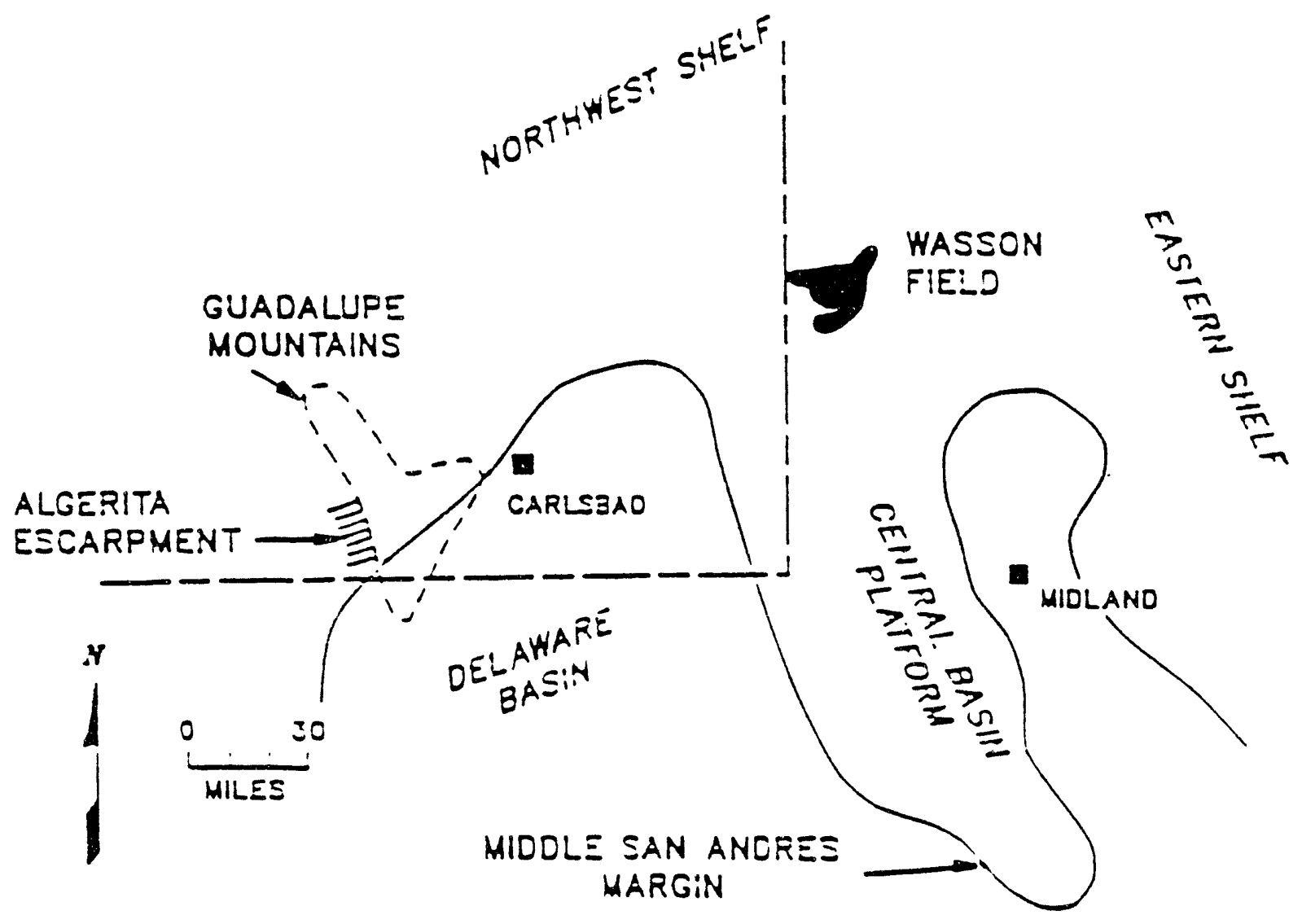

E:g.: Locazon of Algeria escarement and Wasson İeids in Permin Basin larer

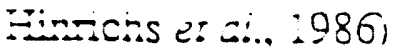




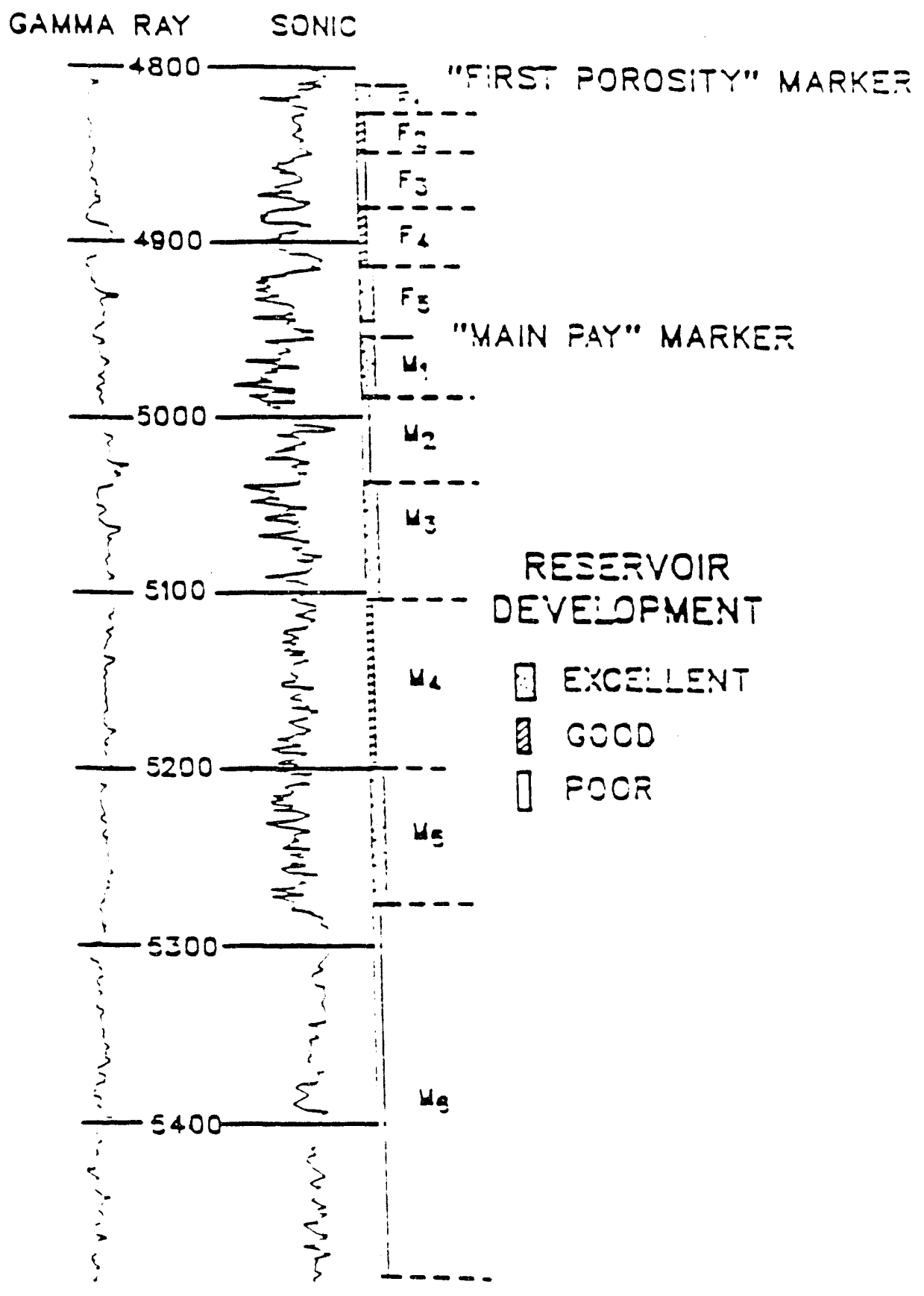

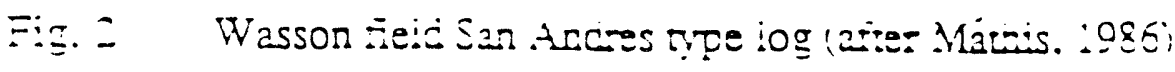




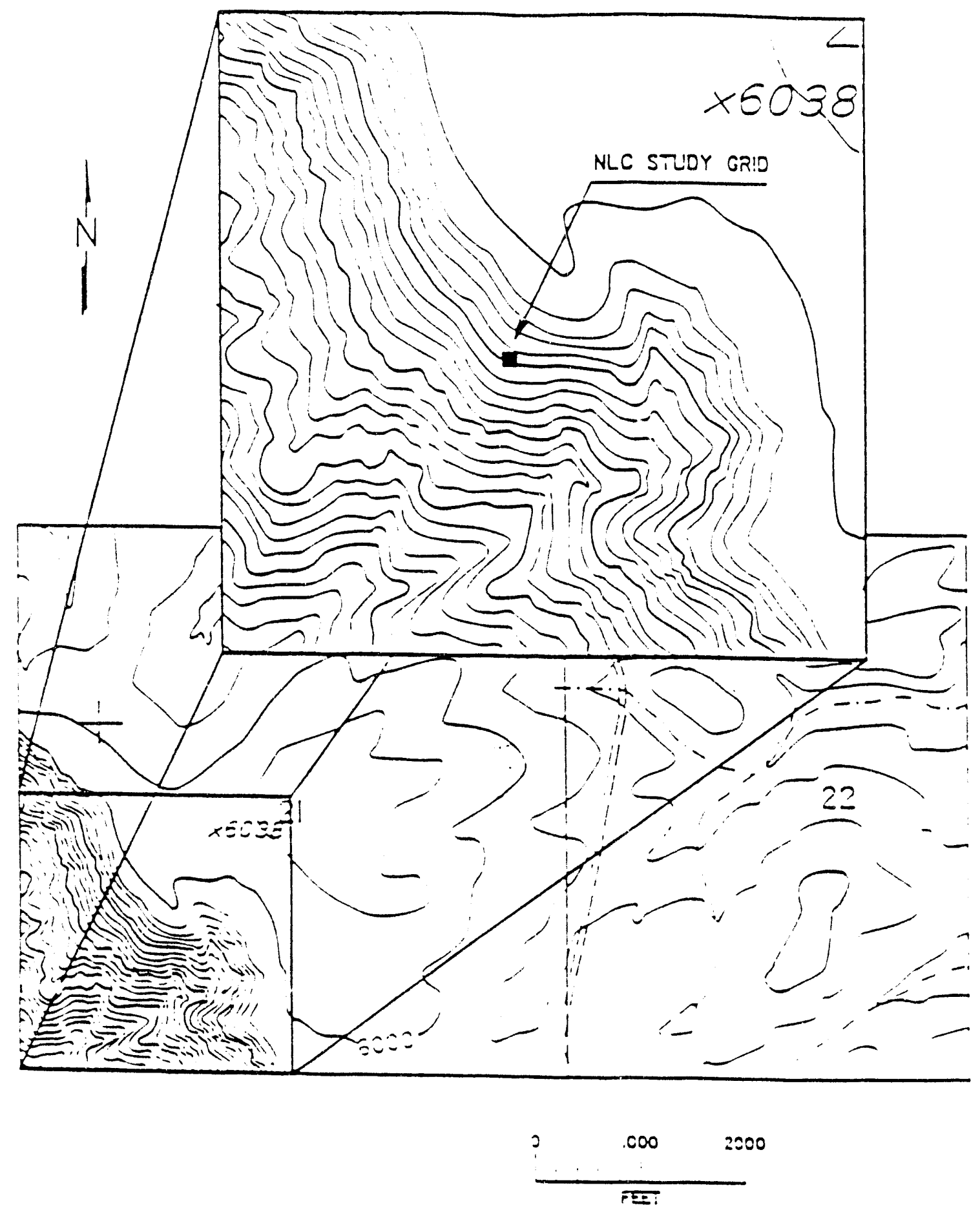

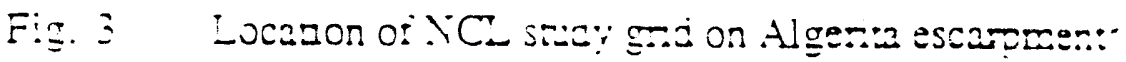




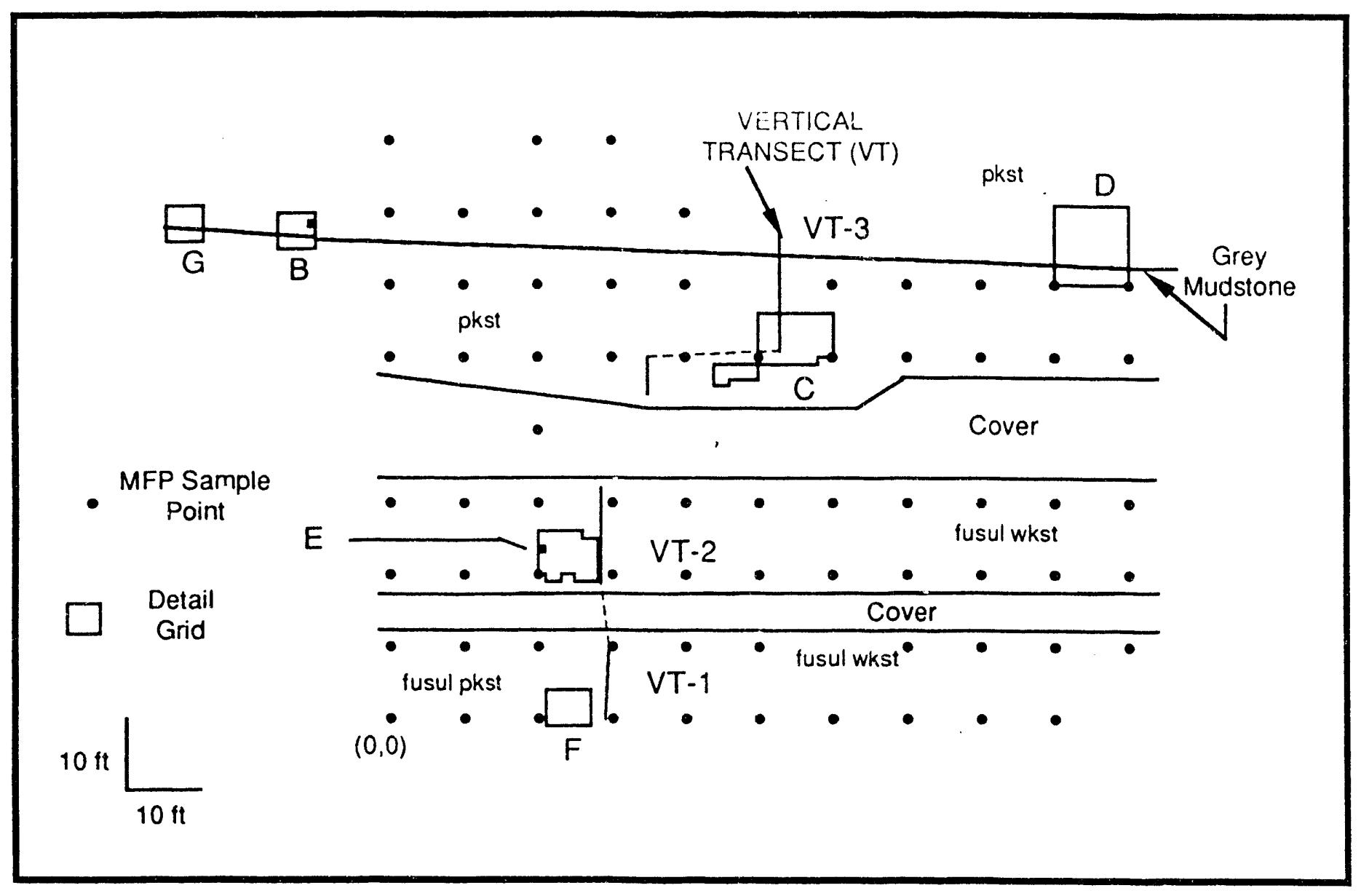

Fig. 4 Grid locations and general geology of NLC study area. Grid A consists of all shown MFP points. VT is the combination of VT-1 through VT-3. 


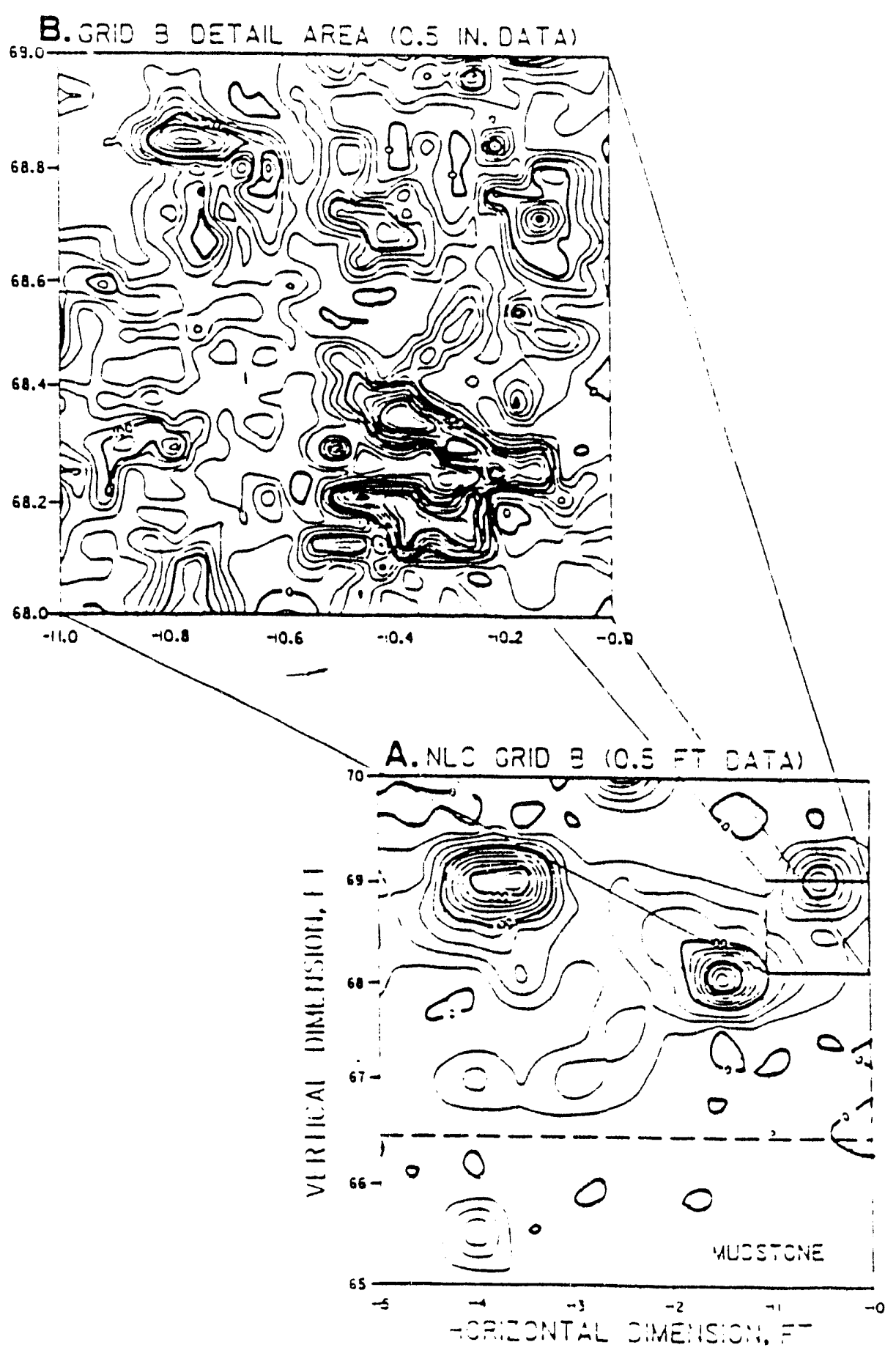

Fig. $\vdots \quad$ Yermeasility contour piots 


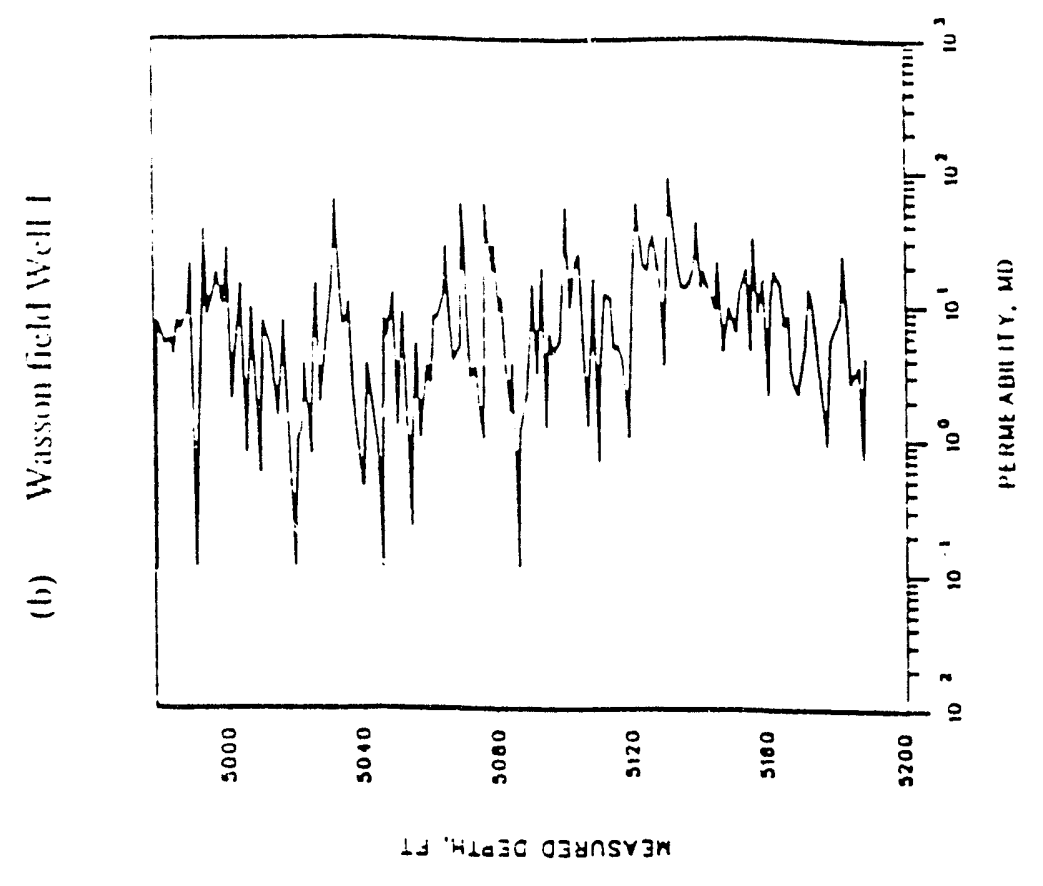

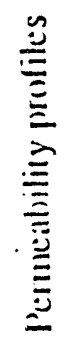

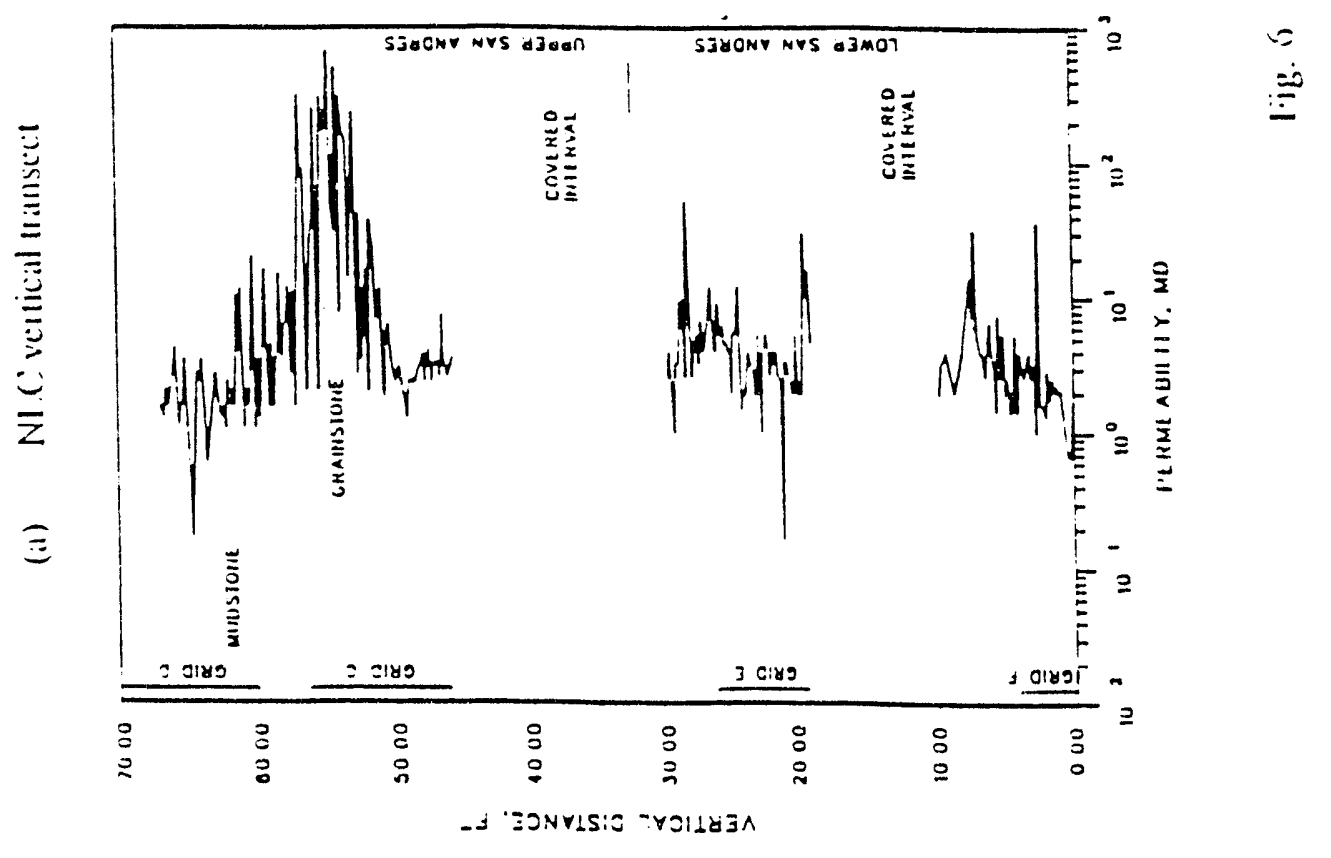




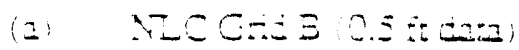

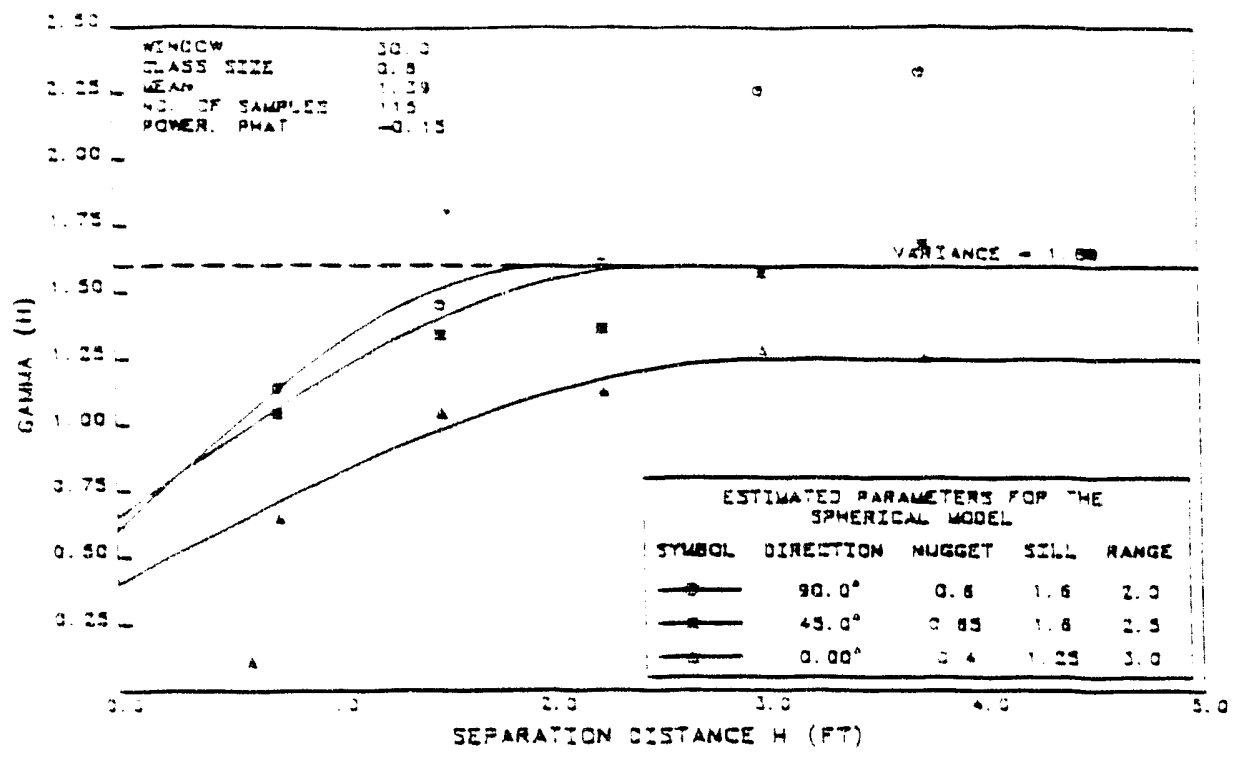

(b) $\operatorname{MLCGidB}(0.5$ in dan)

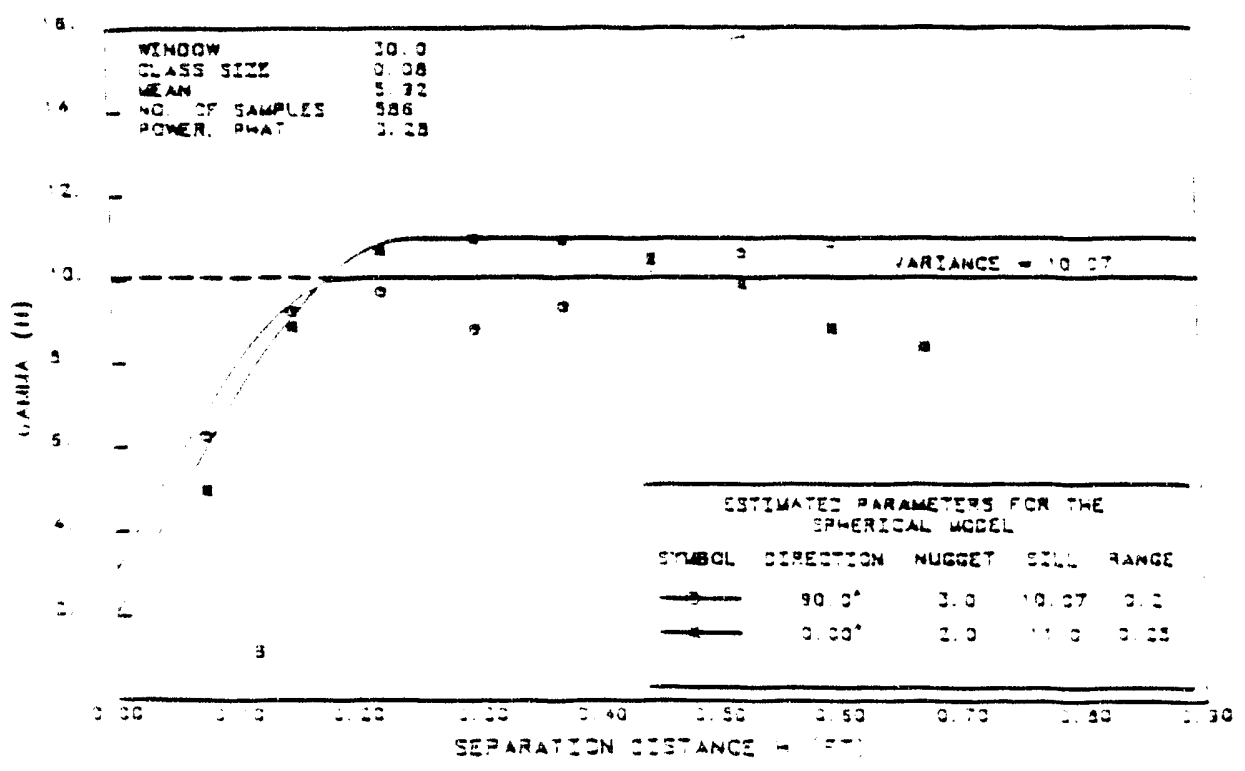

Fig. - Candecvarogras 
(a) NIC ver=cai =ansec: : 2 on 6 and

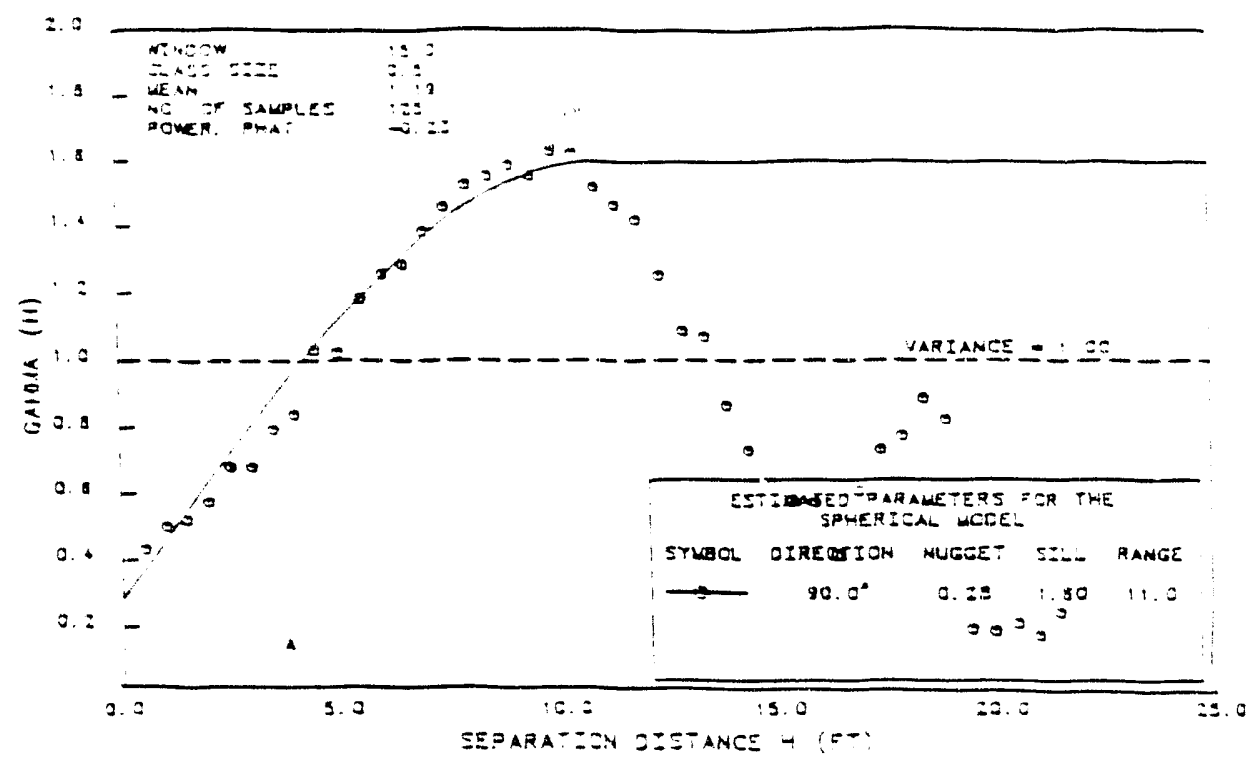

(b) Wasson neid Well : $\mathrm{K}, 1 \mathrm{ft} \mathrm{dra}$

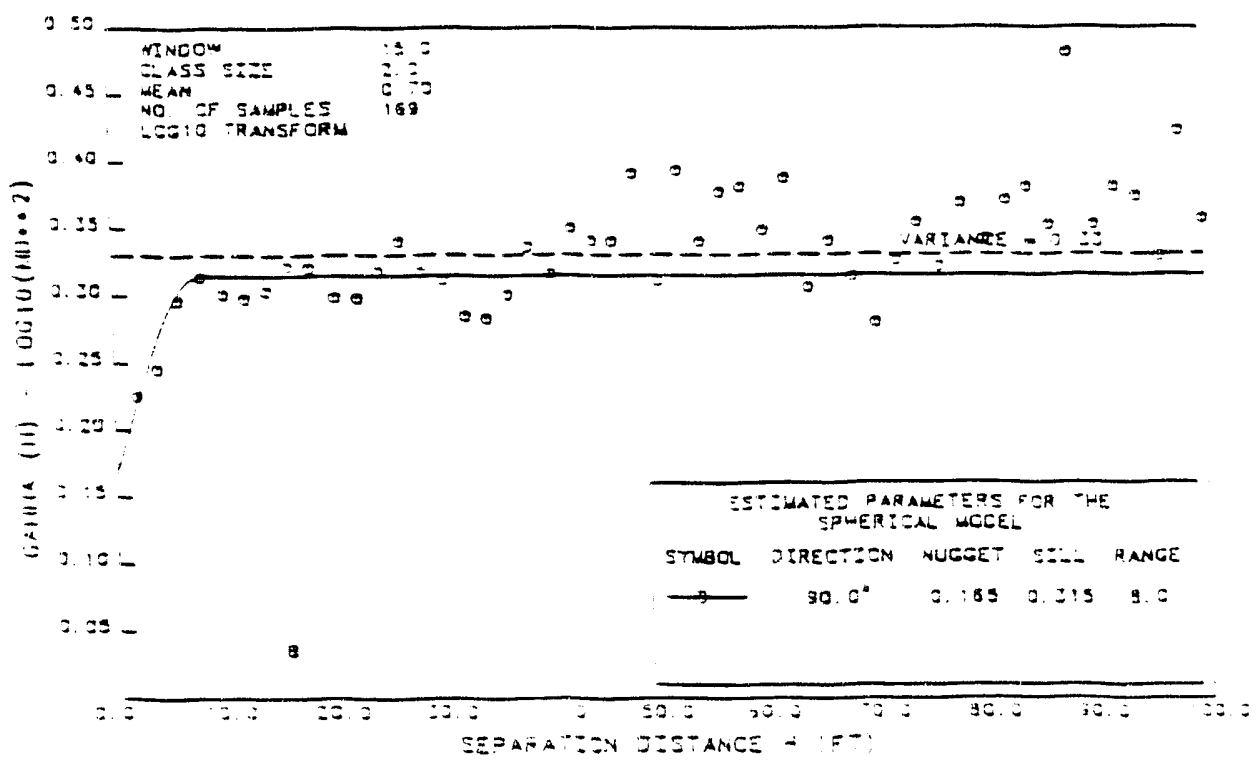

Fig. 3 Calouinted varogs-s 

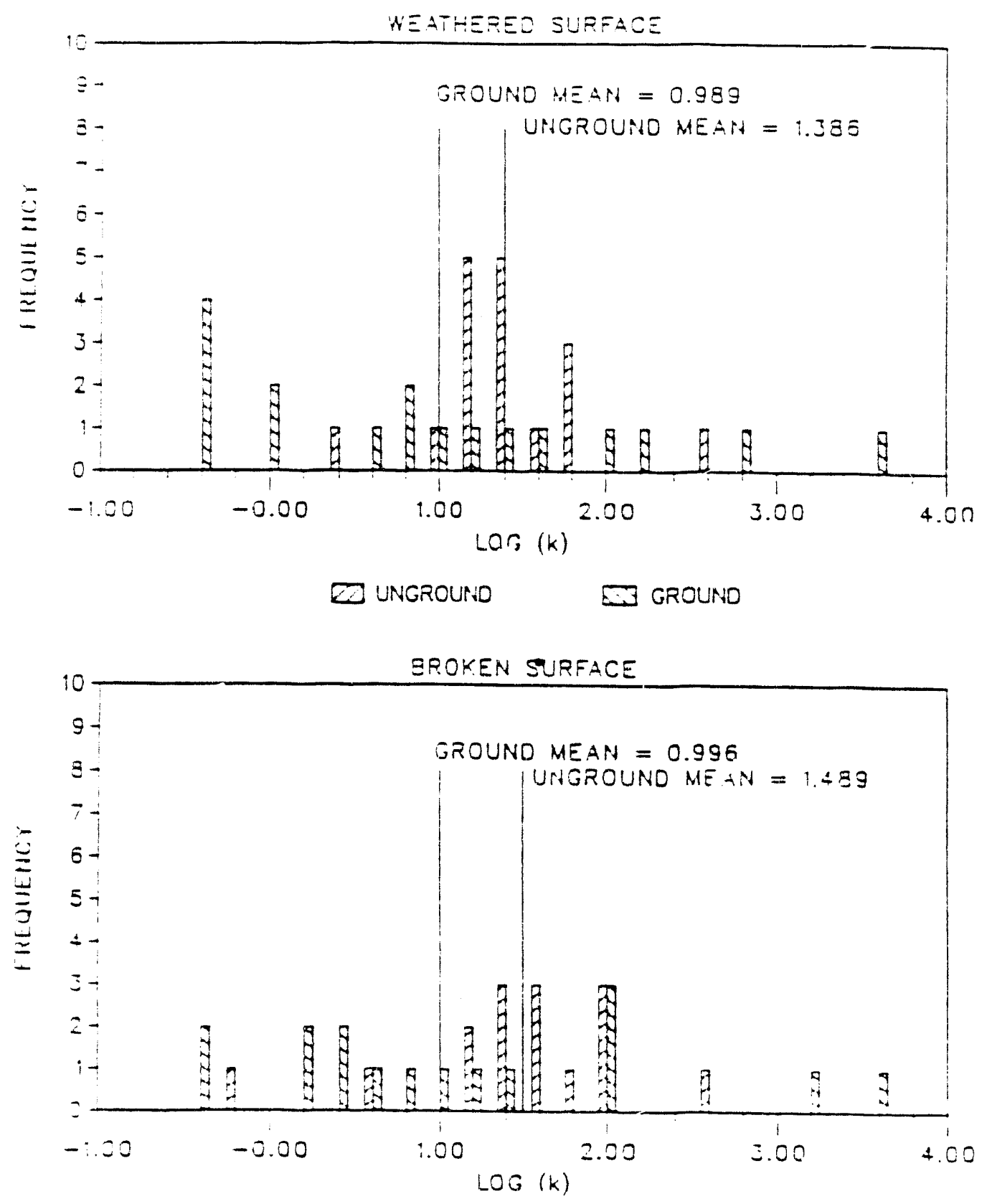

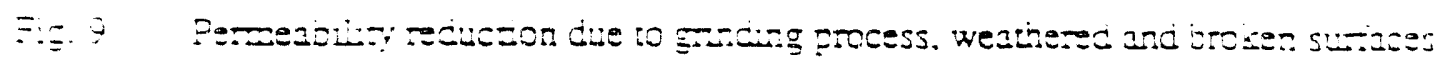




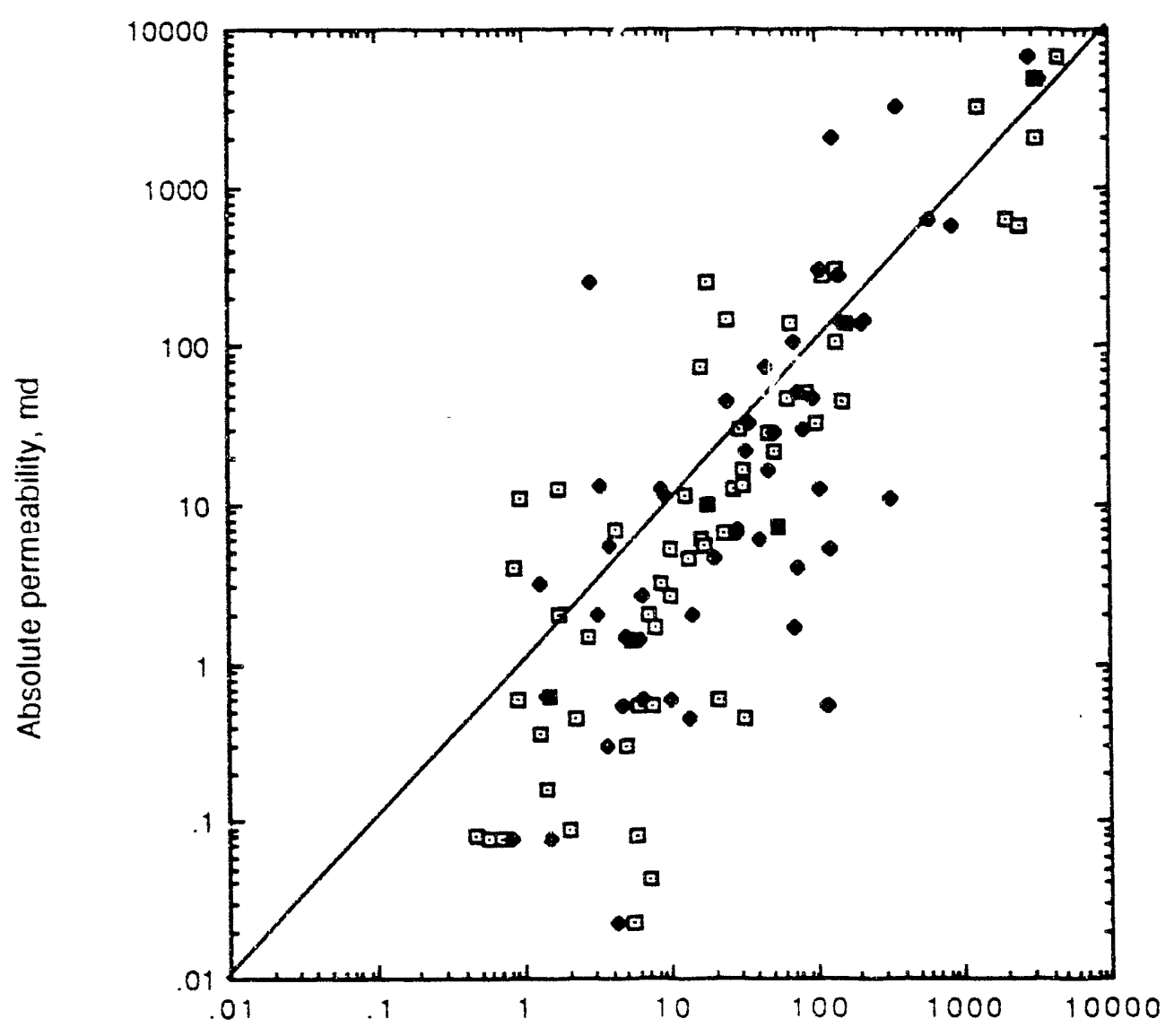

MFP permeability, md

Fig. 10 Comparison of MFP and absolute permeabilitits, NLC core plugs. Open and filled symbols designate measurements on :wo ends of the same core plug. 
Subtask 1c - Lse of Geochemical Flow Modeling to Determine the Geometric Patterns in Porosity and Permeability that Result from Diagenetic Processes, and to Define the Patterns of Permeability in Carbonate Formations and the Occurrence of Calcite Cement Inclusions Caused by both Bacterial Action and Thermochemical Processes.

The approach for this problem is to study reactive flow in the presence of a combination of reactions both in and out of local thermodynamic equilibrium. To proceed along the latter, we have solved a reactive problem for flow through a tube with the intent of using these insights to broaden the work to flow through permeable media. The results of the tube model are to be used in a network simulation to identify circumstances when dissolution or precipitation (calcification) would occur. Thus far we have been able to generate both instabilities and damping in nerwork reactive flow.

We will provide a full report on subtask ic with the final report. 


\section{Subtask 1d - Development of an Experimental Validation Procedure I'sing Simulated Fluid-flow to Quantify the Amount of Geologic Detail Required for a "Satisfactory" Characterization.}

The work under this subtask consists of running a viscously unstable displacement through the deterministic outcrop discussed in Kasap and Lake (1990). We find that such simulations are very expensive and time-consuming, but they do generate different oil recoveries compared to their unit viscosity ratio analog. However, the basic conclusions from the unit mobility ratio study with regards to the importance of geologic phenomena are unchanged. In all cases, the displacement fronts are more erratic, but this effect on the overall recovery is small.

\section{Application to flow in an outcrop study}

\section{Detailed Simulations}

The purposes of the detailed simulations are two fold: the first is to generate a base case to which the results of less detailed simulations using effective relative permeabilities are compared. The second is to see the effect of small-scale heterogeneities in the presence of large scale heterogeneities. The objective was to find out if the small scale heterogeneities will alter the sweep efficiency of the displacement. The large-scale heterogeneities here are the geologic stratification types (grainflow or windripple stratifications). The small-scale heterogeneities (corresponding variation in permeability within a stratification type), on the other hand, are generated randomly from a normal distribution. Both grainflow and windripple stratification types have different means and standard deviations.

The geologically-realistic medium through which we will simulate fluid flow is a portion of the Page Sandstone outcrop. The Page Sandstone is an ancient eolian sand which underlies a large area of the Southwestern U.S. and outcrops in Northern Arizona. This three-dimensional outcrop was surveyed and mapped in excruciating detail by Knight (1986) in conjunction with extensive permeability measurements using a field minipermeameter (Chandier, 1986: Goggin, 1988a). In the next few paragraphs we give a very brief discussion of eolian sands in general, focusing on the Page Sandstone in particular. 


\section{Eolian sands}

Eolian sands on the scale dealt with here consist of four basic stratification types (regions of common sedimentation mechanism). A grainflow type is the result of a sand dune collapsing and flowing to the base of a large dune: a windripple type is a region consisting of sand which has been altered by wind action after deposition; a grainfall type is a region of accumulated wind-blown sand; and interdunes are non-eolian regions which separate distinct episodes of dune migration. Grainfalls are not preserved at Page, but the other three types are present in abundance. The interdunes are rather discrete features which bound compartments consisting of the other two types in varying amounts. Grainflows tend to occur above windripples but the former tend to grade into the latter through a complex series of interfingering tongues. Complete grainflows and windripple, as preserved at Page, are relatively free of laminated (cross bedded) sand lenses, but the large transitions between the two are heavily laminated. Page appears to be free of postdepositional alterations.

\section{The Page sandstone}

Based on over 10,000 in situ measurements, we know that the permeability and associated statistics at Page categorize with stratification type (Goggin, 1988b). Grainflow, windripple and interdune regions have average permeabilities of 3,0.75 and 0.15 Darcies ( 1 Darcy $\cong 1 \mu \mathrm{m}^{2}$ ), respectively (Chandler, 1986). On the scale of the cells used in our problem, major axis of the permeability correlation ellipse is about $0.76 \mathrm{~m}$ oriented generally along the direction of cross-bedding (Goggin , 1988a). The minor axis is about $0.15 \mathrm{~m}$ oriented at right angles to the cross-bedding. All stratification types exhibit about the same coefficient of variation and all appear to contain normal permeability distributions.

The upper panel of Fig. 1 shows a mapping of the Northeast wall of the Page outcrop knob. The entire outcrop contains nearly 100 identifiable units spread out over an areal extent of about 6 acres (Knight, 1986). Computer storage and time limits and the scale of permeability variation limits us to simulating only two-dimensional flow through the portion of the Northeast wall shown in the lower panel of Fig. 1. This portion was chosen because it contains one of only two continuous units (C2) in the outcrop and a highly discontinuous lower zone consisting of more than 20 identifiable units (the Bcomplex). The interdunes are shown as solid bounding surface lines in the lower panel and the grainflows and windripples by the shading. 


\section{The flow field and detailed simulation}

Figure 2 show's only panel B of the grid structure used on the C-B complex. The highly complex internal geometry of the flow field lead us to use a finite element procedure to simulate fluid flow. See Kasap (1990) for details of the simulator.

The size of the element areas was assigned to give good definition within even the smullest unit but aiso so that it was larger than the measured correlation length in permeability. We assigned permeabilities at each element according to the measured means in the above-cited studies. This means that the windripple regions have low permeabilities; the grainflow regions have high permeabilities. The very thin interdune bounding surfaces have very low permeabilities. The elements of the dispersion tensor were assigned according to

$$
\begin{aligned}
& \left.\mathrm{K}_{\mathrm{ii}}=\frac{\left(\alpha_{\ell}-\alpha_{\mathrm{l}}\right)}{\phi|\mathrm{v}|}\right) \mathrm{v}_{\mathrm{i}} \mathrm{v}_{\mathrm{i}}+\frac{\alpha_{\mathrm{l}}|\mathrm{v}|}{\phi} \\
& \left.\mathrm{K}_{\mathrm{ij}}=\frac{\left(\alpha_{\ell}-\alpha_{l}\right)}{\phi|\mathrm{v}|}\right) \mathrm{v}_{\mathrm{i}} \mathrm{v}_{\mathrm{j}}
\end{aligned}
$$

where ii refers to the diagonal elements and $\mathrm{ij}$ refers to off-diagonal elements of the dispersion tensor. $\alpha_{\ell}$ and $\alpha_{t}$ are the longitudinal and transverse dispersivities for that particular element. Equation (1) is, strictly speaking, valid only for isotropic media (Bear), but we are unaware of an alternative. The dispersivities were calculated from the coefficient of permeability variation $\left(C_{v}\right)$ and correlation length $(\lambda)$ according to

$$
\alpha_{\ell}=C_{v}^{2} \lambda
$$

Arya et al. (1988) show that dispersivity is constant only if the size of the flow field is larger than the correlation length, a restriction which imposes (along with computer limits) a lower bound on the size of element areas. Goggin et al. (1989) show that correlation lengths for homogeneous and the heterogeneous regions are different. Therefore we assigned dispersivities as a function of heterogeneity. The measure of heterogeneity was taken to be proportional to the magnitude of the off-diagonal elements of the effective permeability tensor. First, we calculated the possible maximum off-diagonal element of the permeability tensor for a given ratio between grainflow and windripple permeabilities. 
Then we assigned a dispersivity to that block between maximum $\left(k_{x y}=k_{x y m}\right)$ and minimum $\left(k_{x y}=0\right)$ values according to the magnitude of the off-diagonal element of its atectue permedbility tensor.

$$
\alpha_{i i}=C_{v}^{2}\left[-\frac{\left(\lambda_{h e}-\lambda_{h o}\right)\left|k_{x y i}\right|}{\left|k_{x y m}\right|}+\lambda_{h o}\right]
$$

$\mathrm{k}_{\mathrm{xym}}$ was calculated as a function of permeability ratio and cross bedding angle. See Kasap for details $. C_{v}=0.33 \lambda_{\text {ho }}=0.15 \mathrm{~m}$ and $\lambda_{\text {he }}=0.76 \mathrm{~m}$ have all been estimated from field measurements (Goggin, 1988a). Each bounding surface contains at least one finite element node.

The simulation field shown in Figs. 1 and 2 is intensively deterministic. The entire flow field consists of 11,520 finite elements and 11,277 nodes. It is very likely the most detailed and realistic flow field ever simulated, particularly with respect to the geology, and the tensorial nature of permeability and dispersivity. The flow field is unrealistic, however, in that it is not an actual reservoir, it is two-dimensional and it is in reality not separated from the units above and below it as the presumption of no-flow boundaries at these locations enforces. The latter objections are the subject of future work; nevertheless, insights gained here should apply to two-dimensional flow simulations and vertical-sweep considerations which are very common in practice.

Although our average element size was $0.4 \times 1 \mathrm{~m}$ (the element sizes at second order bounding surfaces were $0.12 \times 1 \mathrm{~m}$ ), 11520 elements were not enough to describe all the geologic details exactly. In the midsections of the layers, the transition from grainflow to windripple regions are not sharp and the elements in these regions contain heterogeneities in the form of windripple laminations at $35^{\circ}$ to the horizontal.

\section{Characterization of the outcrop}

The results from the detailed simulation showed that the detail obtained by representing the field with 11520 elements was not necessary at least for the flow field studied here. Figure 3, showing tracer concentration profiles at different injection times, indicates that the the flow behavior of the field is much simpler than our expectations. By inspecting Fig. 3. it is possible to characterize the field as being composed of four distinct layers: one at the top with very high permeability and mainly composed of grainflow structures: below that is a low permeability layer with mainly windripple stratification: the third is high permeability layer with party wind ripple and partly grainflow structures and 
the last one is again a pure windripple layer. The presence of the compartmentalization caused by the interdunes does not affect the vertical sweep efficiency very much; nor does the permeability anisotropy because a simulation of fluid flowing in the opposite direction of Fig. 3 shows essentially the same vertical sweep efficiency. Evidently, fluid avoids low permeability windripple and transitional areas where the anisotropy is most pronounced.

After identifying the layers, we decided to simulate the fluid flow in the same field with less detail. Figure 4 shows only panel B of the second grid structure. This time we represented the field with 2437 elements and 2452 nodes. The average element area was four times larger than the one in the detailed simulation. The bounding surfaces are not represented as elements and it was assumed that they were part of windripple laminations. Figure 4, comparing detailed and coarse simulation results, indicates that main flow paths in these two representations are the same. Although Fig. 5 shows that the amount of fluid flowing in each layer is slightly different in two cases (slightly more fluid goes to the third layer in the absence of the bounding surfaces), the main features of layers are still preserved. In these simulations, the ratio of grainflow to windripple permeability was 4 . In both cases the local or microscopic mixing zone is much smaller than the gross or megascopic mixing zone (Arya, 1988). The specific representation of dispersion, then, is unimportant for this case.

The outcrop simulations also show that the most effective factor for determining sweep efficiency is the ratio of the permeabilities of the grainflow to windripple units. Figure 3 shows tracer concentrations in the case of permeability ratios of 4 and 20 at 0.55 pore volumes injected. Figure 3 indicates that when the permeability ratio is 20 , most of the injected fluid goes to the upper layer leaving the lower portion of the field unswept. The breakthrough in the second case was much earlier ( 0.49 pore volumes injected) than the first case $(0.77$ pore volumes injected). Figure 6 shows oil-cuts versus time and indicates a large difference in the vertical sweep efficiencies of those two cases. We conclude from these results that the most important feature in determining vertical sweep efficiency in eolian sands is the permeability contrast between the grainflow and windripple regions and the relative frequency of the former.

A normal distribution for both stratification types was used because the results of the field study indicate a distribution that approaches the normal distribution as long as we stay within a stratification type (Goggin et al., 1989). For each element, one grainflow and one windripple permeability are obtained from the same random number by using different means and coefficient of variations for grainflow and windripple permeabilities. $\widetilde{k}_{\mathrm{GF}}=$ 
3.0 D. $\widetilde{k}_{W R}=0.75 \mathrm{D}$. and $C_{v_{G F}}=C_{v_{W R}}=0.25$ were all obtained trom previous study (Goggin et al.). Once grainflow and windripple permeabilities are calculated for each. element, by using the method developed by Kasap and Lake (199()) the permeability tensor of each element is calculated as a function of grainflow and windripple volumetric fractions, local permeabilities, cross-bedding angle and random numbers.

The finite element simulator was run to simulate fluid flow in this outcrop field under unfavorable mobility ratio conditions with those two sets of permeability distributions. Figure 7 compares the front positions obtained from the simulations with deterministic and stochastic permeabilities within a stratification (grainflow or windripple) at $0.55 \mathrm{PV}$ injected. The area contacted by the injected fluid and the recovery will not be altered by assigning random permeabilities. Random permeabilities will, however, cause a slightly larger local mixing zone which can be seen in Fig. 7b. In both cases the local or microscopic mixing zone is smaller than the gross or megascopic mixing zone. Comparing the profiles in Figs. 3 and 7 suggests that mobility ratio is also important.

\section{Conclusions}

Several conclusions can be drawn from these simulations: (1) in the presence of the large-scale heterogeneities, uncorrelated small scale heterogeneities do not affect the sweep efficiency and the recovery; (2) the specific representation of dispersion remains unimportant on this scale; (3) mobility ratio is important regardless of the representation of heterogeneity.

\section{References}

Arya, A., T.A. Hewitt, R.G. Larson and L.W. Lake, "Dispersion and Reservoir Heterogeneity," .SPE Reservoir Engineering, Feb. 1988, 139-148.

Bear, J., Dynamics of Fluids in Porous Media American Elsevier Publishing Co., Inc., New York City, 1977.

Chandler, M.A., "Depositional Controls on Permeability in an Eolian Sandstone Sequence, Page Sandstone, Northern Arizona," M.A. Thesis, University of Texas at Austin, 1986. 
Guggin, D.J., "Geolagically Sensible Modeling of Permeability Patterns in Eolian Deposits: Page Sandstone (Jurassic) Outcrop, Northern Arizona," Ph.D. Dissertation, The Lniversity of Texas at Austin, 1988.

Goggin, D.J., M.A. Chandler, G. Kocurek, L.W. Lake, "Patterns of Permeability in Eolian Deposits," SPE Formation Evaluation, Vol 3, No. 2, June 1988, 297-306.

Goggin, D.J., M.A. Chandler, G. A. Kocurek and L.W. Lake, "Permeability Transects in Eolian Sands and Their Use in Generating Random Permeability Fields," SPE Paper 19586 to be presented at the 1989 SPE Annual Technical Conference and Exhibition, San Antonio, Oct. 8-11.

Kasap, Ekrem, "Fluid Flow in Eolian Deposits: An Outcrop Study of Page Standstone," Ph.D Dissertation, The University of Texas at Austin, 1990.

Kasap, Ekrem and Larry W. Lake: "An Analytical Method to Calculate the Effective Permeability Tensor of a Grid Block and Its Application in an Outcrop Study," SPE Eormation Evaluation, June, 1990.

Knight, J.B., "Eolian Bedform Reconstruction: A Case Study from the Page Sandstone, Northern Arizona," M.A. Thesis, University of Texas at Austin, 1986.

\section{Nomenclature}

$\mathrm{C}_{\mathrm{v}}$

$\alpha_{\ell}$

$\alpha_{\mathrm{t}}$

$\mathrm{K}$

Greek

$\alpha$

$\alpha_{\ell}$

$\alpha_{\mathrm{t}}$

$\lambda$

$\phi$
Coefficient of variation of the velocity field

Longitudinal dispersivity [ $=] \mathrm{L}$

Transverse dispersivity $[=] \mathrm{L}$

dispersion Tensor $[=] \mathrm{L}^{2} / \mathrm{t}$

$\begin{array}{ll}\alpha & \text { Rotation angle } \\ \alpha_{\ell} & \text { Longitudinal dispersivity [=] L } \\ \alpha_{t} & \text { Transverse dispersivity [=] L } \\ \lambda & \text { Correlation length [=] L } \\ \phi & \text { porosity }\end{array}$


Subscripts

he heterogeneous region

ho homogeneous region

i element of the permeability tensor or the grid scheme

m maximum

$x, y \quad$ simulation coordinate system

1,2 regions with different permeabilities within the simulation block

\section{List of Figures}

Figure

1 Northeast wall of Page Sandstone outcrop and B-C units with discrete grainflow windripple regions

22679 finite elements

3 Concentration profiles at different times

4626 finite elements

5 Concentration profiles from the simulations with two grid systems

6 Effluent histories from simulations with different permeability ratios

7 Displacing fluid front locations and mixing zones ( $0.3 \leq$ mixing zone concentration $\geq 0.7$ in detailed simulations at $0.55 \mathrm{PVI}, \mathrm{M}=10$

(a) Uniform permeabilities within grainflow or windripple structures

(b) Normally distributed random permeabilities within the structures 


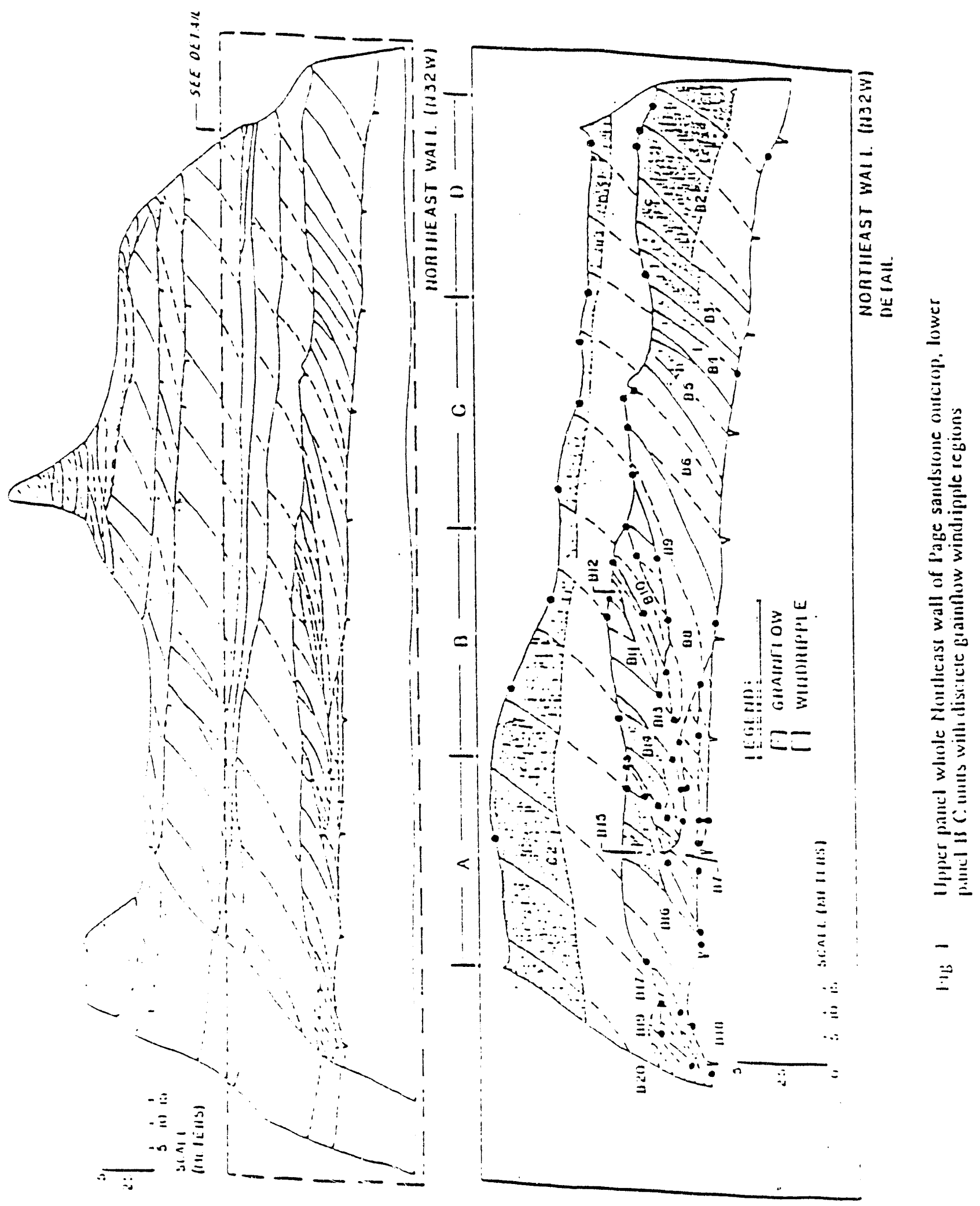




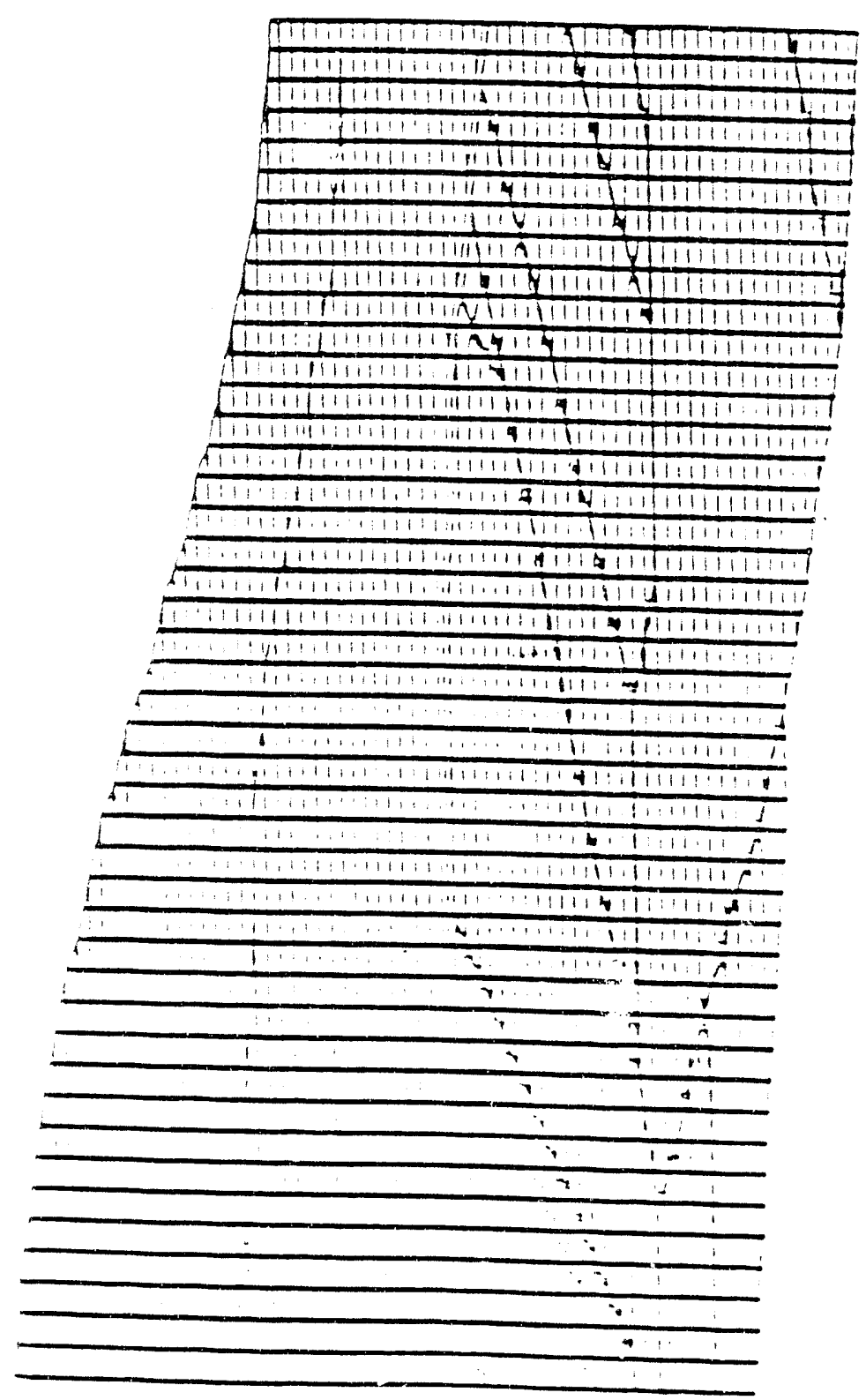

三 

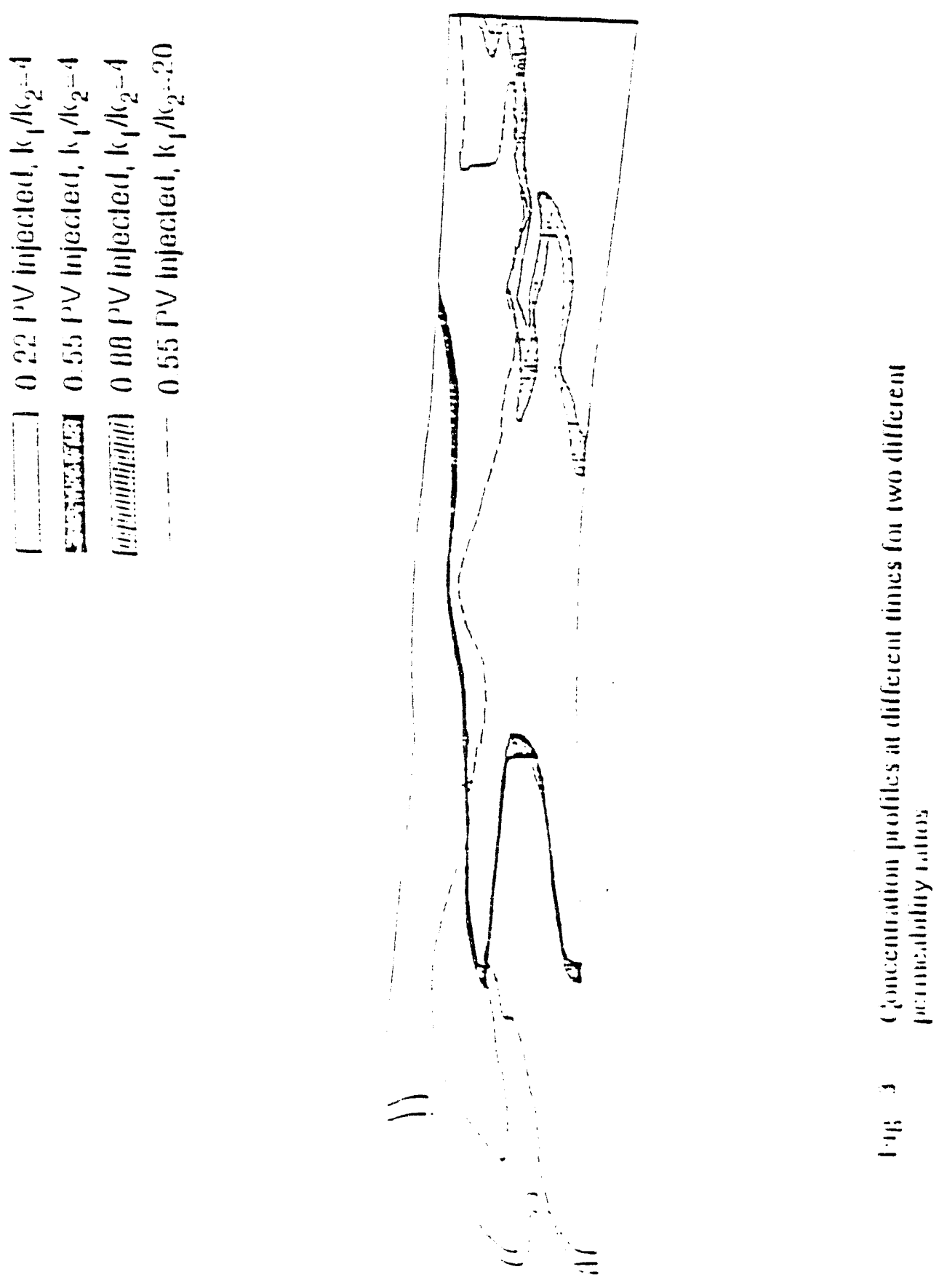

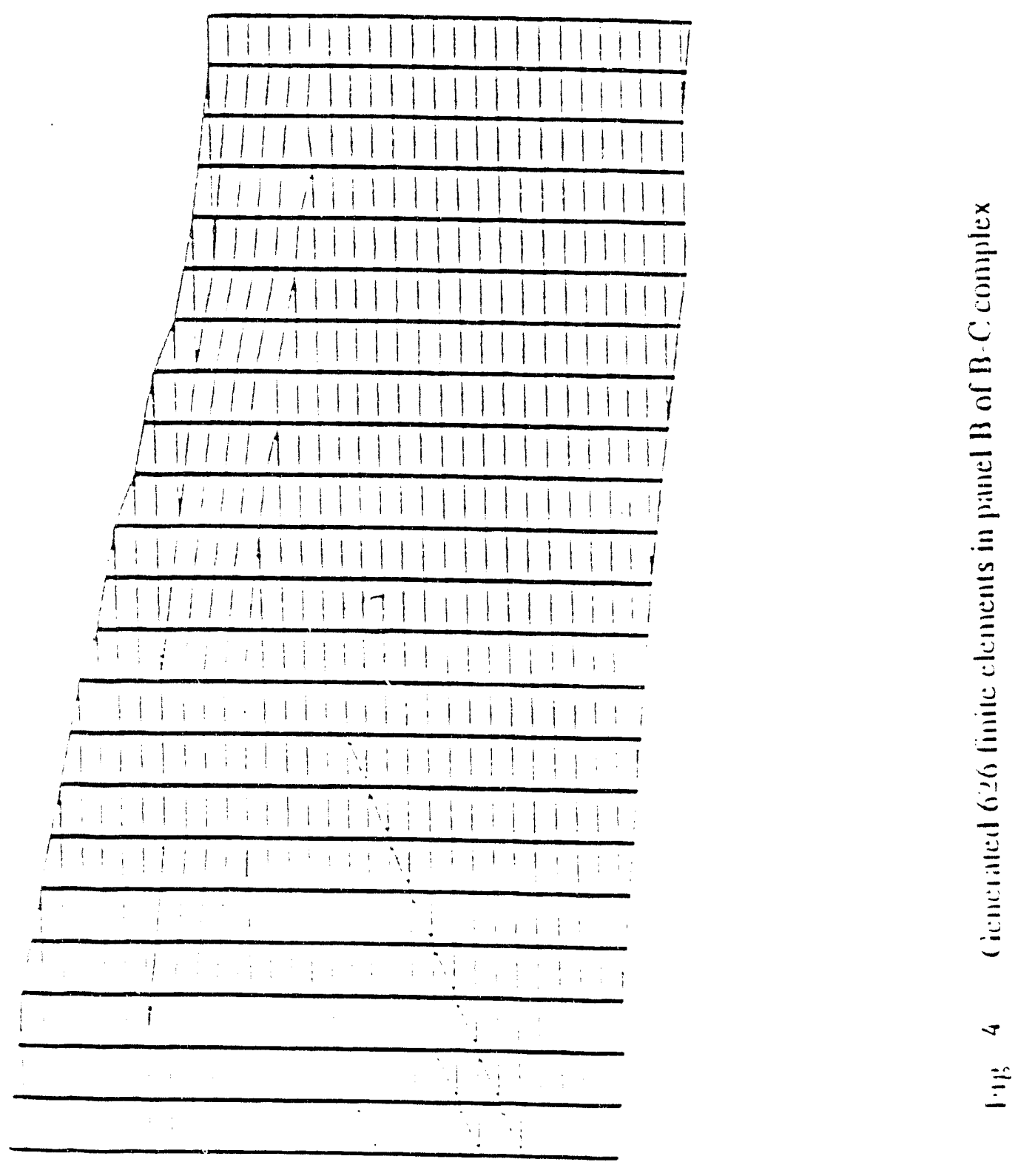


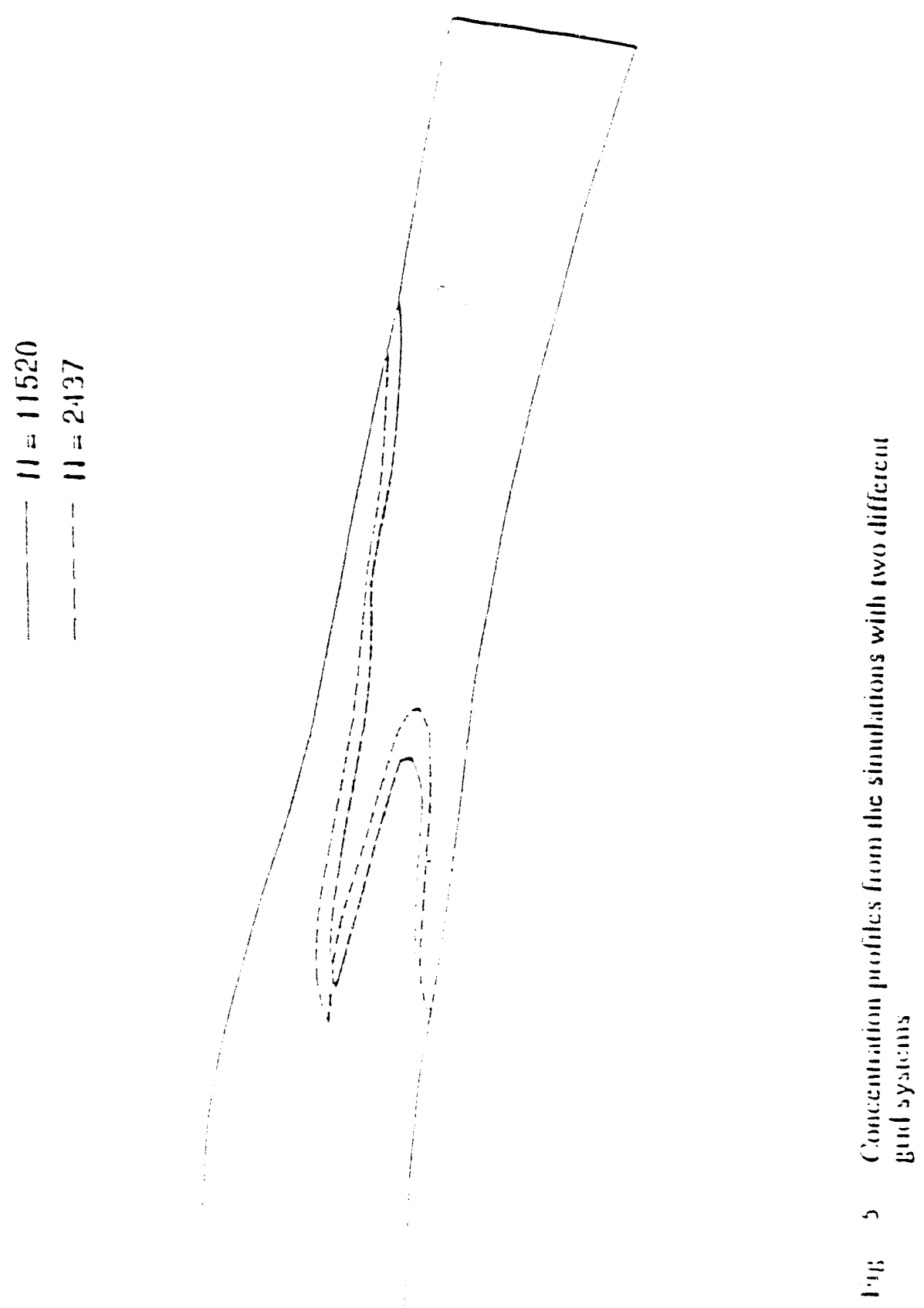




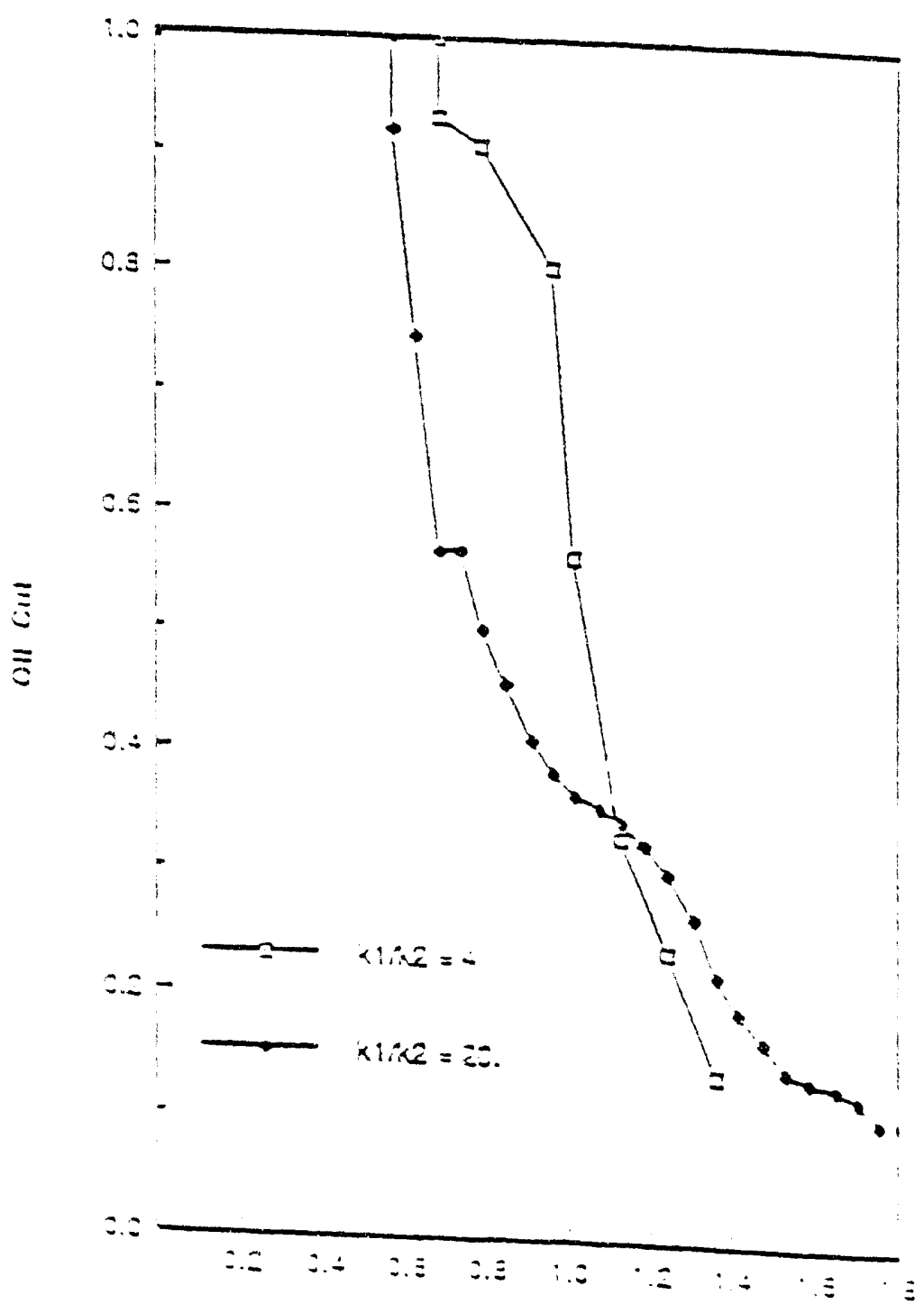

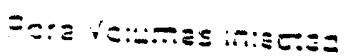

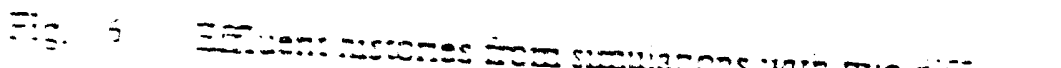

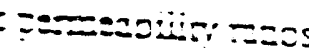



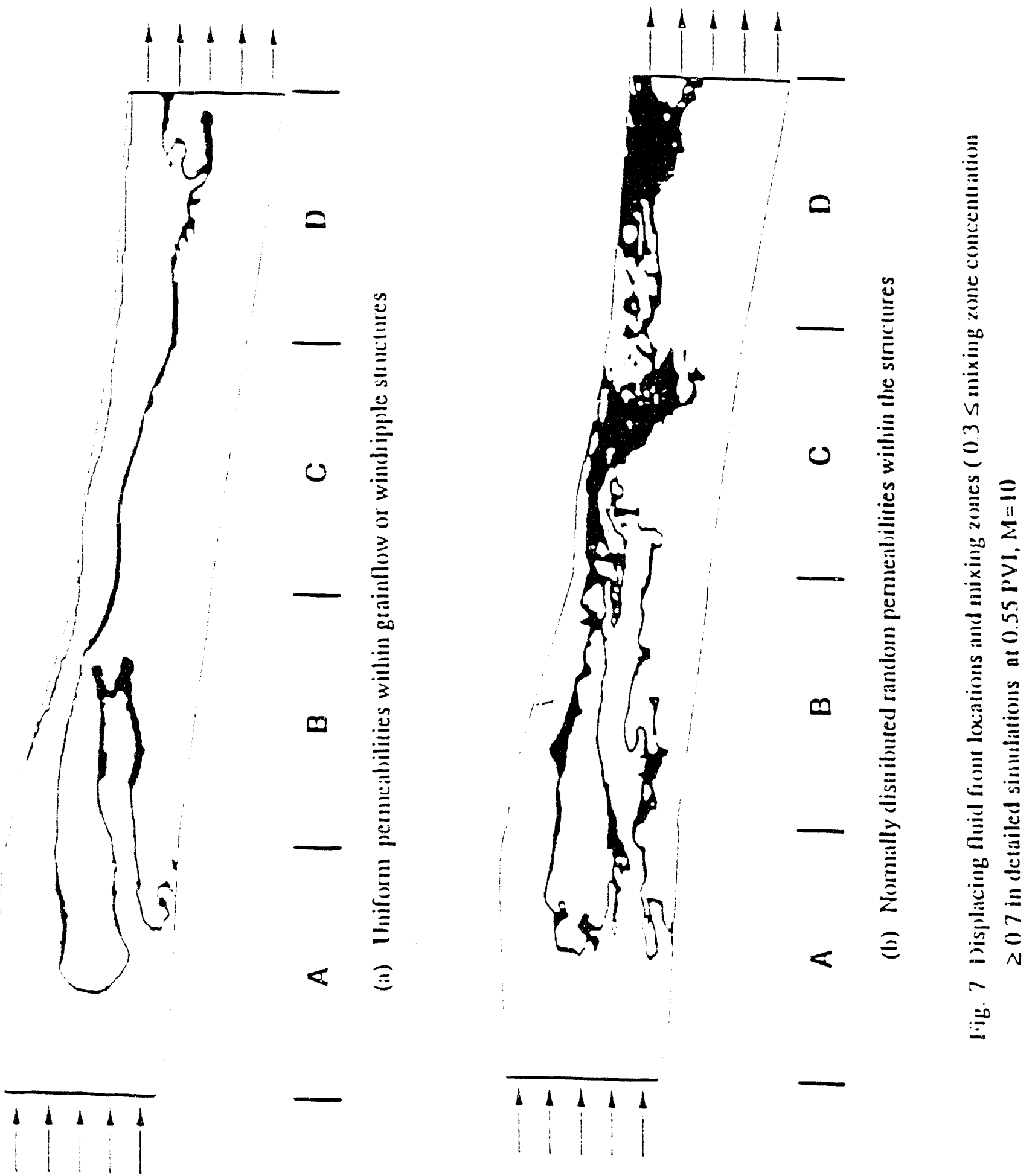


\title{
TASK 2 - MODELING AND SCALEUP OF CHEMICAL FLOODING
}

\author{
Subtask 2a - Perform Single-Well Tracer Studies to Estimate Reservoir \\ Properties Other Than Those of Residual Oil Saturation
}

\section{Introduction}

A two-dimensional radial coordinate option has been added to The University of Texas chemical flooding simulator (UTCHEM). The radial option assumes a horizontal reservoir with a constant pressure outer boundary and a rate specified well at its center. It retains the same formulation as the cartesian-only version of UTCHEM and therefore was added without major changes to the existing code. While all of the complex physical process models required for accurate simulation of chemical flooding have been retained, the current use of the radial option is the investigation of single-well tracer flow. The most common example of this is the single-well backflow tracer method for measuring residual oil saturation. However, the primary focus of this project is not the further study of this particular test, but of bar.kflow tracer methods to determine other reservoir characteristics, such as wettability

The mathematical formulation of the radial option and its implementation in UTCHEM has been completed (Descant, 1989). Comparisons of simulation results to an analytical solution of the radial convection-dispersion equation show that the simulator accurately models dispersive radial flow, to the point that numerical dispersion control may not be the limiting factor in grid block size. A study of the effects of communicating layers on the residual oil saturation determining tracer test reveals that the effect of transverse dispersion in single-we!l backflow tracer tests will be significant in instances of large permeability distributions. A preliminary investigation into the effects of capacitance on single-well backflow tracer tests without a soak period shows that, while the results are sensitive to reasonable changes in the capacitance parameters, interpreting the effects of capacitance on an acrual test may be difficult.

Only an example of our results for the effect of transverse dispersion ur the single well backflow tracer test are given below. Other results can be found in the thesis of Descant (1989). 


\section{Discussion}

One use of the radial option is the simulation of single-well, backflow tracer tests for determination of residual oil saturation (Tomich et al., 1973). The theory of the test and its procedure is well documented (Deans and Majoros, 1980b). A slug of water containing an ester (primary tracer) is injected into a reservoir that is at or near residual oil saturation, followed by more water. The well is shut in, allowing the ester to partially hydrolyze into alcohol (secondary tracer). The well is then produced. Because the ester partitions into the immobile oil much more than the alcohol does, the ester concentration peak will be chromatographically retarded compared to that of the alcohol. This separation is a function of residual oil saturation. Usually, a third non-partitioning non-reacting tracer is included to check material balance.

If certain idealized assumptions are made, including no dispersion and negligible injection and production times, residual oil saturation may be directly calculated from the produced tracer concentration histories. In most instances, however, histories must be matched by a numerical model in order to estimate residual oil saturation.

Several finite-difference simulation studies of the residual oil saturation tracer test have been published. In these studies, non-communicating layers are assumed in layered reservoirs. Therefore, potentially important mechanisms such as transverse dispersion are neglected. In this brief study of the residual oil saturation tracer test, no attempt at matching or analyzing field data from the literature has been made. Instead, the sensitivity of simulations of the test to layering and crossflow is examined, since this has not been published.

The reservoir and tracer parameters chosen for this study, taken from a test analyzed by Bragg et al. (1976) and Deans and Majoros (1980a,b), are given in Table 1. The ethyl acetate to ethanol reaction is assumed to be of first order and irreversible with $[E T O H]=k[E T A C]$ where $k$ is the reaction constant with units of days ${ }^{-1}$ and the concentration units are vol\%. Drift velocity was negligible, as indicated by produced tracer concentration histories. Both groups simulated the test as a homogeneous reservoir and estimated the residual oil saturation to be about $13 \%$. However, the test was not simulated exactly by either group. Bragg et al. (1976) pointed out that the simulated production history of the ethyl acetate (primary tracer) was normalized downward to match the field data peak value, in order to compensate for loss of the tracer to lift gas in the wellbore. They apparently did not make this correction with the other tracers, which were alcohols. Deans and Majoros $(1980 \mathrm{a}, \mathrm{b})$ stated that the absolute value of the tracer concentration histories are not so important as the location of the peaks (as functions of produced 
volume). They therefore disregarded the actual injected concentrations and arbitrarily scaled their simulation results to match the data peaks, so that both the primary and secondary tracer histories were corrected. In simulations with UTCHEM, a good match of the material balance tracer history was possible, but the peaks of the simulated histories of both the primary and secondary tracers were at least twice as great as their respective field data peaks. Deans and Majoros did not report the scaling factors they used.

A base case simulation run was made with UTCHEM using the same input as given in Table 1. A grid refinement study showed that 18 radial grid blocks with a constant $\Delta r$ of $2.1 \mathrm{ft}$ gave results comparable to 60 grid blocks with a $\Delta \mathrm{r}$ of $0.6 \mathrm{ft}$. The 60 grid block run took 77.2 CPU seconds, while the 18 grid block run took $17.8 \mathrm{CPU}$ seconds. A longitudinal dispersivity of $1 \mathrm{ft}$ best fit the material balance $t$ acer data. This radial grid and value of longitudinal dispersivity were used in subsequent multilayer runs.

Figures 1, 2, and 3 show the effects of transverse dispersivity on a five-layer reservoir. The layers are of equal thickness and have permeabilities of $800,500,1400$, 200 , and $1100 \mathrm{md}$. Results for transverse dispersivities from 0 to $1 \mathrm{ft}$ are presented. This case was more sensitive to transverse dispersivity than a two-layer case (Descant, 1989). This was due not only to the greater number of layers and larger permeability variation, but also to the layer arrangement. A large difference in permeability between adjacent layers is needed to allow enough dispersive mixing between layers to significantly affect produced concentrations (Descant, 1989). However, in such cases large errors in the measured residual oil saturation are possible due to the traditional neglect of transverse dispersion and such cases thus merit a more careful analysis.

\section{References}

Bragg, J.R., L.O. Carlson, and J.H. Atterbury: "Recent Applications of the Single-Well Tracer Method for Measuring Residual Oil Saturation," paper SPE 5805 presented at the Third SPE Symposium on Improved Oil Recovery, Tulsa, OK, Mar. 22-24, 1976.

Deans, H.A. and S. Majoros: "Single-Well Chemical Tracer Test Simulator Handbook: Improved Accuracy and Interpretation for Residual Oil Saturation Measurement," U.S DOE Report BC/20006-14, June, 1980.

Deans, H.A. and S. Majoros: "The Single-Well Chemical Tracer Method for Measuring Residual Oil Saturation," U.S. DOE Report BC/20006-18, Oct., 1980.

Descant, F.: "Simulation of Single-Well Tracer Test - (UTCHEM)," M.S. thesis, U. of Texas, Austin, Aug., 1989. 
Tomich, J.F., R.L. Dalton, H.A. Deans, and L.K. Shallenberger: "Single-Well Tracer Method to Measure Residual Oil Saturation," J. Pet. Tech. (Feb. 1973), 211-218.

Table 1. Reservoir and Tracer Data for Residual Oil Saturation Test

\begin{tabular}{ll} 
Thickness & $20 \mathrm{ft}$ \\
Porosity & 7.34 \\
Permeability & $\varepsilon: 00 \mathrm{md}$ \\
Water saturation & 0.13 \\
Wellbore radius & $0.25 \mathrm{ft}$ \\
Slug size & $1000 \mathrm{bbl}$ \\
Total injection & $1980 \mathrm{bbl}$ \\
Injection rate & $1000 \mathrm{bbl} / \mathrm{day}$ \\
Total Production & $3900 \mathrm{bbl}$ \\
Production rate & $650 \mathrm{bbl} /$ day \\
Primary to secondary reaction rate & \\
\multicolumn{1}{l}{ constant (first-order) } & 0.015 days $^{-1}$
\end{tabular}

Tracer Injected Concentration Partition Coefficient (oil/water

Methanol (material balance) $0.5 \mathrm{vol} \%$ (slug only) 0

$\begin{array}{lll}\text { Ethyl Acetate (primary) } & 1.0 \mathrm{vol} \% & 6.7\end{array}$

Ethanol (secondary tracer) $\quad 0 \quad 0$

\section{List of Figures}

Figure

1 Effect of Transverse Dispersivity on Material Balance Tracer for Five-Layer Case

2 Effect of Transverse Dispersivity on Primary Tracer for Five-Layer Case

3 Effect of Transverse Dispersivity on Secondary Tracer for Five-Layer Case 


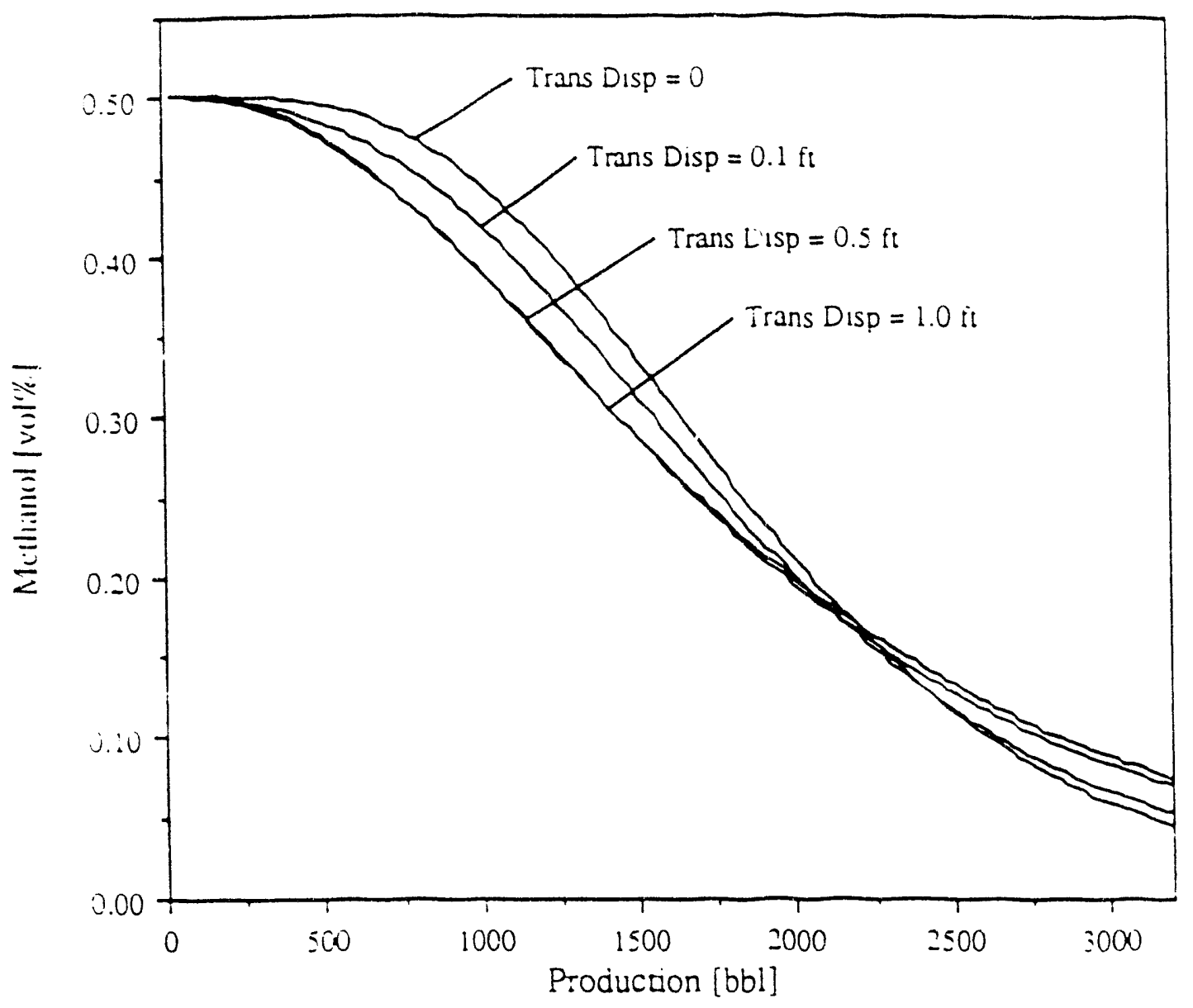

Fig. 1 Effect of Transverse Dispersivify on Materiaj

Baiance Tracer tor Five-Laver Case 


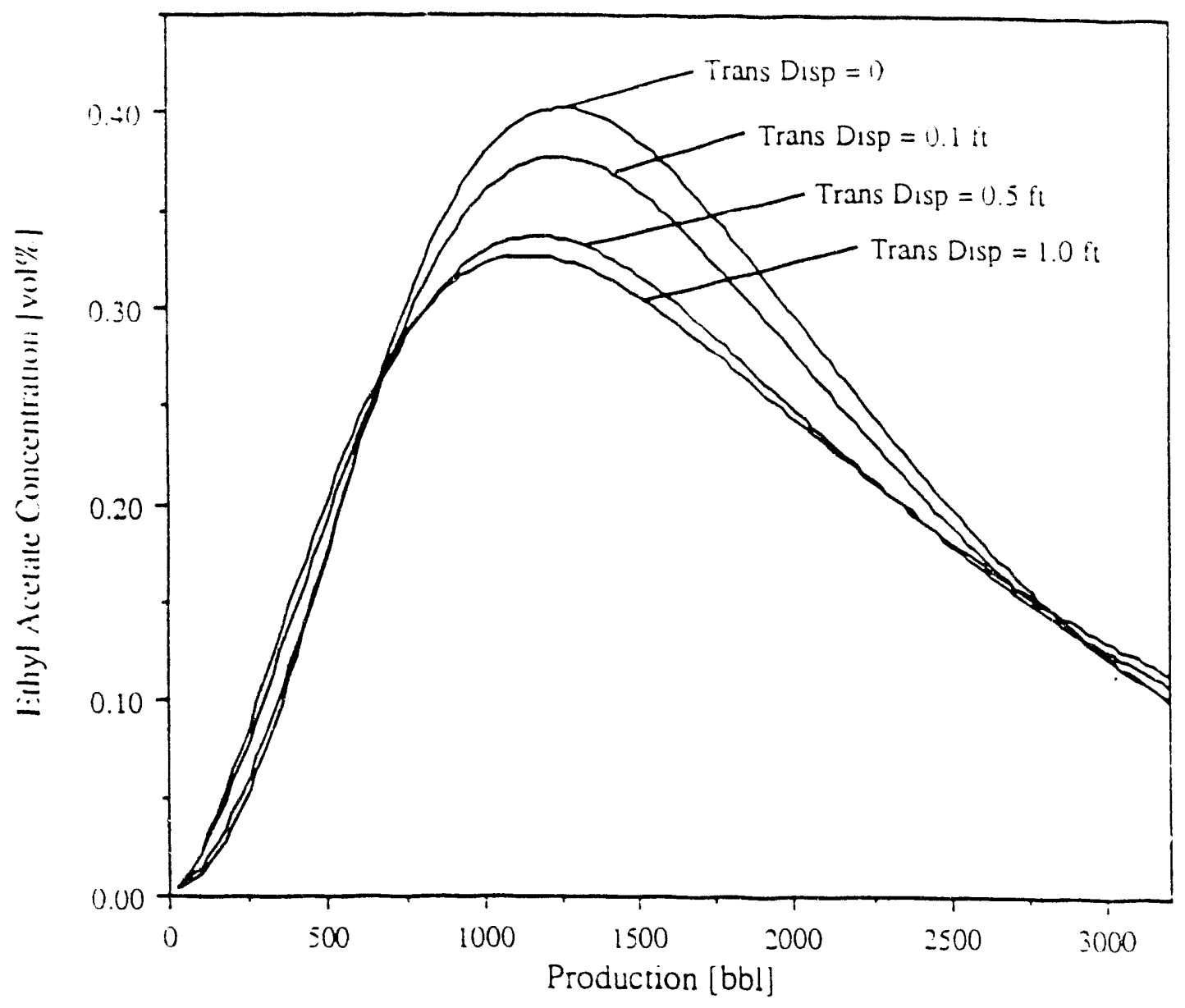

Fig. 2 Effect of Transverse Dispersivity on Primar:

Tracer for Five-Laver Case 


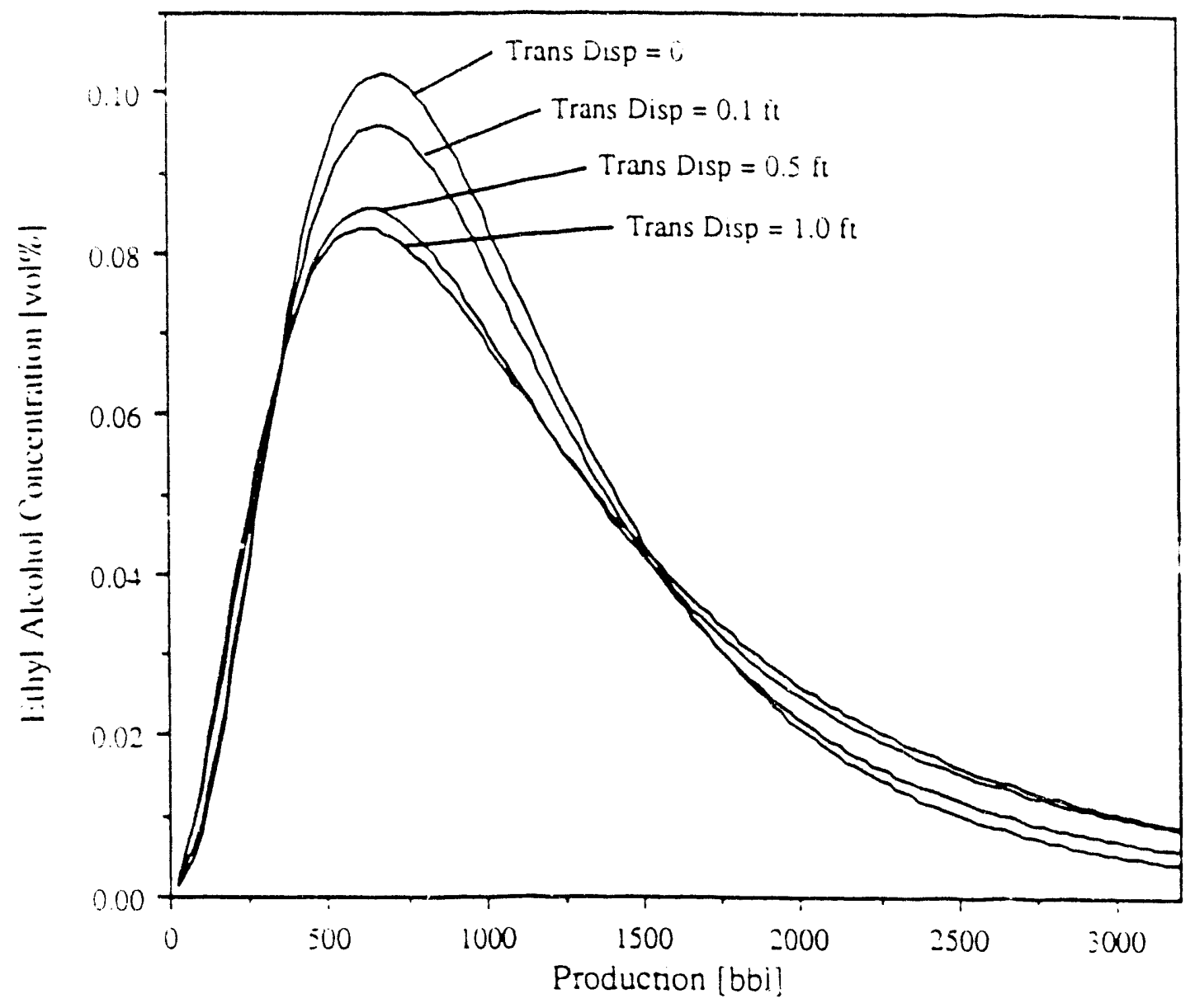

Fig. : Efiect of Transverse Dispersivity on Seconcary

Tracer for Five-Layer Case 
Subtask 2b - Measure the In-Situ Behavior of Gels Formed While Flowing. in Permeable Media

\section{Introduction}

The competition for chromium between xanthan biopolymer and the resident clays in Berea sandstone cores has been examined. Xanthan requires the $\mathrm{Cr}^{3+}$ ion to form gel while the resident clays remove the chromium from the bulk solution by a cation exchange process. These two competing factors are examined through corefloods and computer simulation to determine the amount of chromium actually available for crosslinking.

Through a series of Berea corefloods, chromium retention was examined without the presence of polymer using a $2 \mathrm{wt} \% \mathrm{NaCl}$ brine as the aqueous solution. Finally, several polymer-gel floods were conducted using varying concentrations of chromium and xanthan. Chromium and polymer retention and $\mathrm{pH}$ as well as the pressure drop due to gel formation were analyzed.

The retention of chromium was found to be large and on the order of the cation exchange capacity of the clays in the sandstone. This causes slow transport of the $\mathrm{Cr}^{3+}$, due to its consumption by the clays, and a decreasing effectiveness of the gel indicated by a decreasing reduction in permeability with length. This study points to the need to conduct experiments with porous media containing clay and for better modelling of the complex phenomena occurring under conditions of interest for field applications. We illustrate these results below with only one of our experiments. Others can be found in Garver's thesis (1988).

\section{Discussion}

Simulation and experimental results for $\mathrm{pH}, \mathrm{Cr}^{3+}$ : polymer and pressure drop for Experiment E-8.0 are shown in Figs. 1 through 4. Complete input and other details can be found in Garver's thesis (1988). Only the overall aspects related to $\mathrm{Cr}^{3+}$ transport will be discussed here.

The cation exchange capacity used to simulate this experiment was $0.036 \mathrm{meq} / \mathrm{ml}$ $\mathrm{PV}$. This value is not large enough to account for the entire loss of the $\mathrm{Cr}^{3+}$ nor does the shape of the sirnulated curve (Fig. 2) agree with the experimental results. We did not attempt to history match the experimental curve by increasing thr. CEC because, first. the varue used is representative of this batch of Berea sandstone and, secondly, the additional 
loss of $\mathrm{Cr}^{3+}$ is likely to be a result of precipitation of $\mathrm{Cr}(\mathrm{OH})_{3}$ and perhaps other reactions as well.

The overall agreement between simulated and experimental polymer concentration (Fig. 3) is good, but the experimental values show an erratic behavior not understood but thought to be related to the flow of the gel. Gel was visually observed in produced samples after 2.35 PV.

The simulation results clearly show that the inclusion of $\mathrm{H}^{+}-\mathrm{Cr}^{3+}-\mathrm{Na}^{+}$ionexchange equilibria in our model was insufficient to adequately model all of our experimental observations. These simulation results should therefore be taken as only $a$ preliminary and incomplete effort to model this gelation process. However, they do show that the very late breakthrough of $\mathrm{Cr}^{3+}$ can be accounted for by including the cation exchange of the $\mathrm{Cr}^{3+}$ with the clays. Our (1987) only other attempt to model a gelation coreflood experiment was for the clean coreflood experiment reported by Hubbard $e t$ al. (1986). The agreement in that case was better, but the greater complexity of our own experiments points the way for a need for improved modelling of this process. Even at this stage, however, simulation can be a useful diagnnstic tool for both lab and field cases.

\section{References}

Garver, F.J.: "The Competition for Chromium in a Berea Core Between Xanthan Biopolymer and the Resident Clays," M.S. thesis, U. of Texas, Austin, Dec., 1988.

Hubbard, S., L.R. Roberts, and K.S. Sorbie: "Experimental and Theoretical Investigation of Time-Setting Polymer Gels in Porous Media," paper SPE/DOE 14959 presented at the SPE/DOE 5th Symposium on Enhanced Oil Recovery, Tulsa, OK, April 2023, 1986.

Pope. G.A., L.W. Lake, and K. Sepehrnoori: "Modelling and Scale-up of Chemical Flooding," First Annual Report for October 1985-September 1986 prepared for the U.S. Department of Energy under Contract No. AC19-85BC10846, Bartlesville, OK, July, 1987. 


\section{List of Figures}

\section{Figure}

1

2

3

4 Simulated and Experimental Pressure Drop (Total) vs. Pore Volumes injected for Experiment E-8.0-Measured over the Entire 6 Inches of the Core 


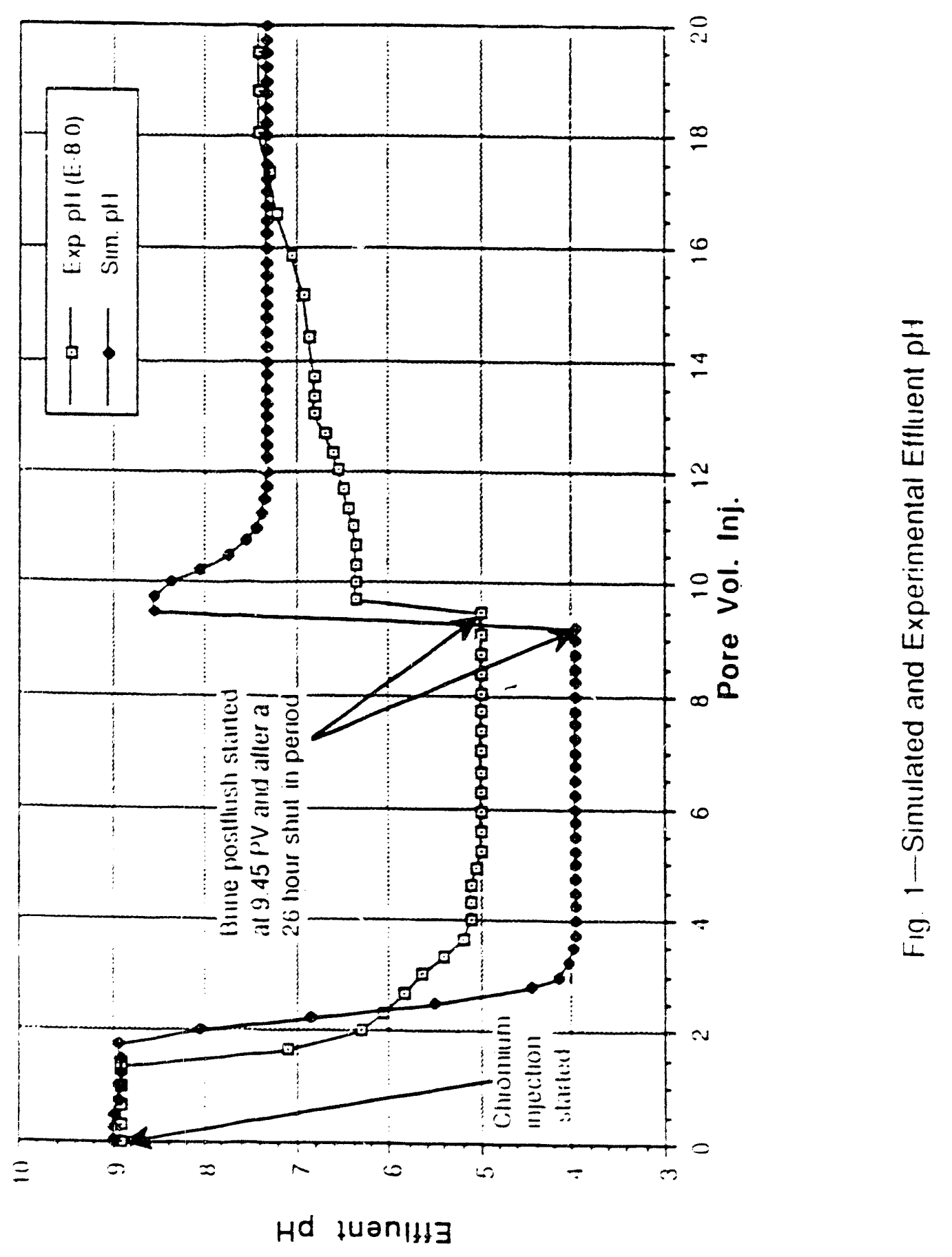




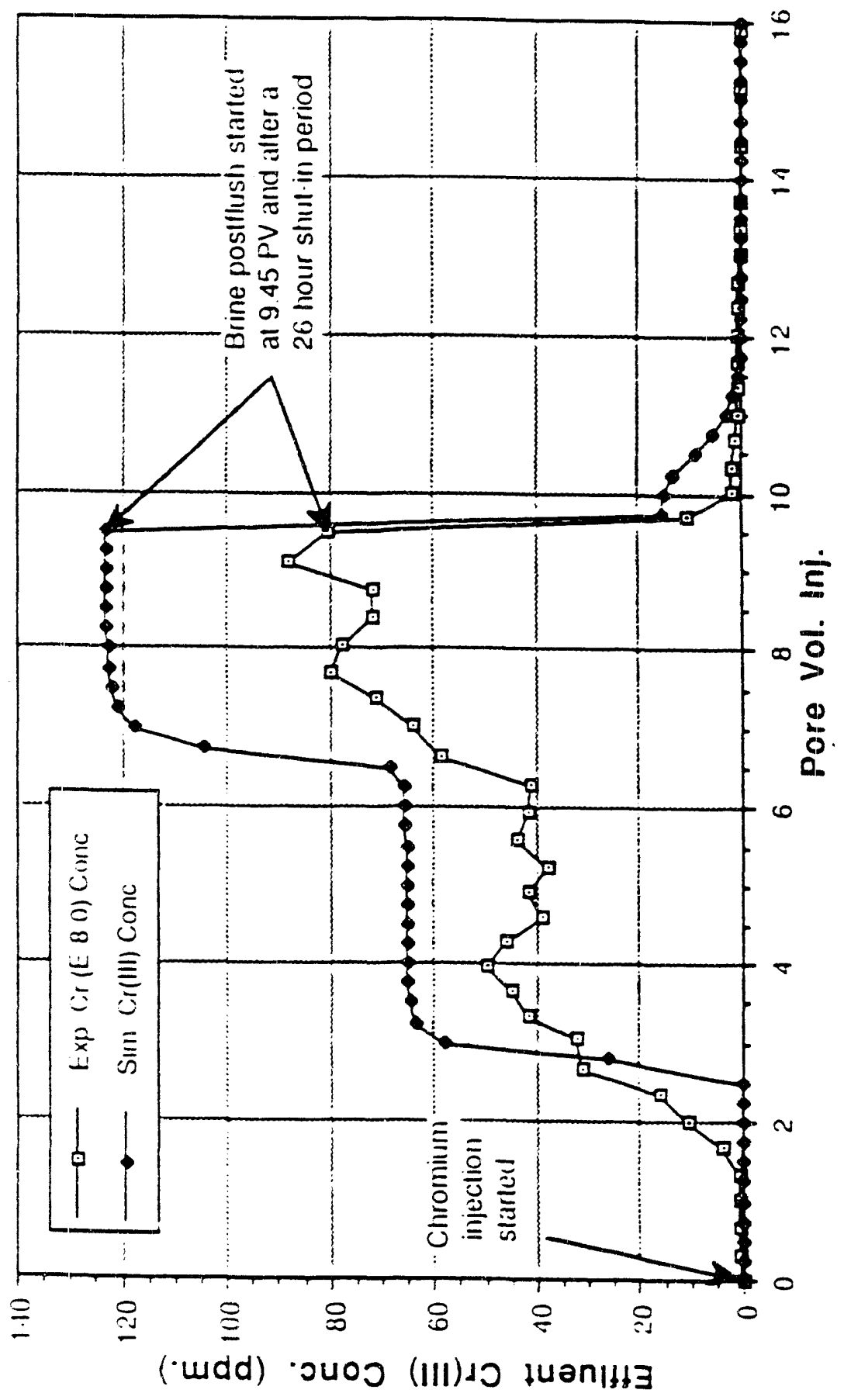

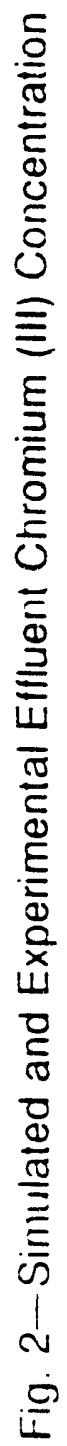




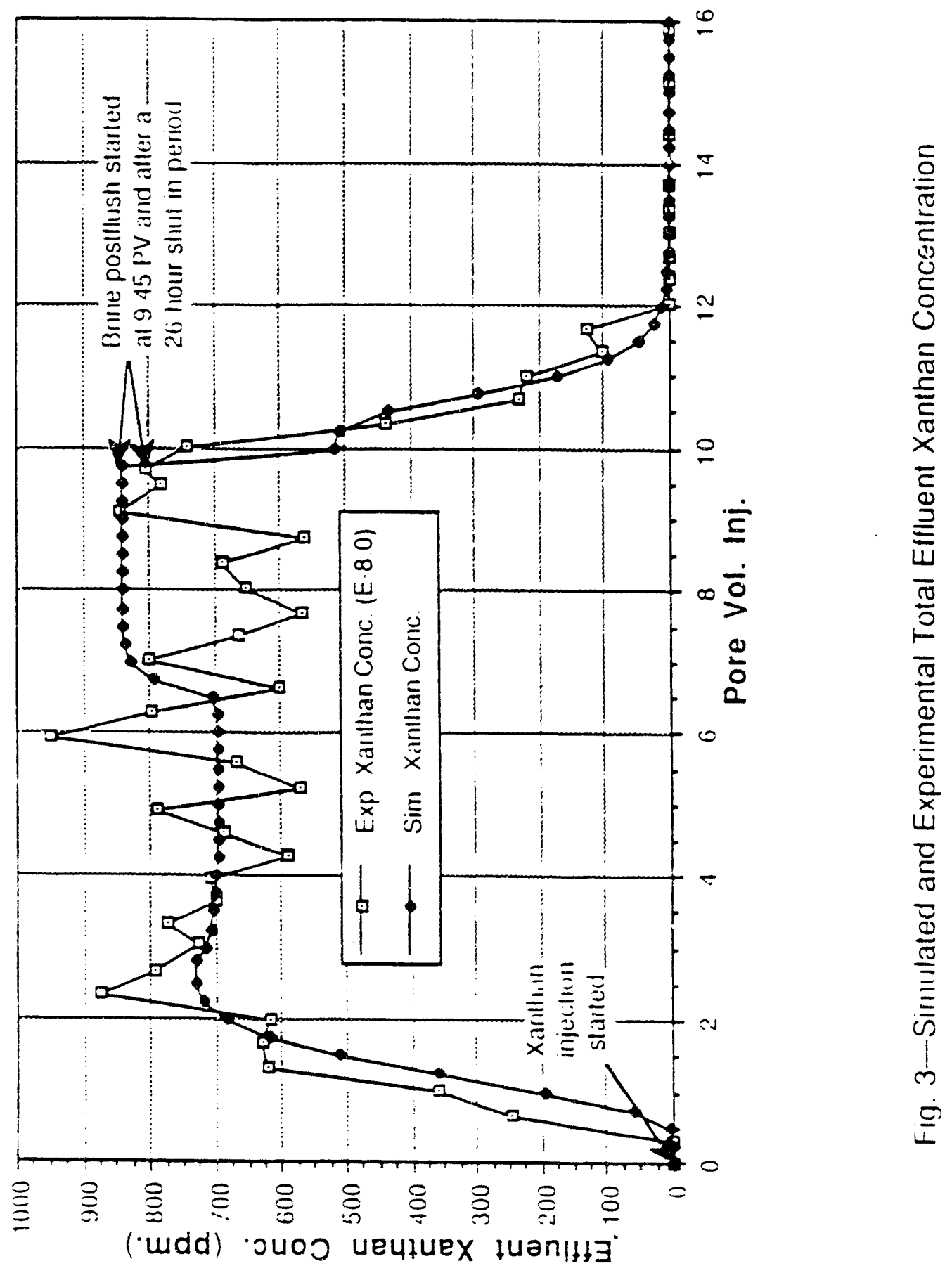




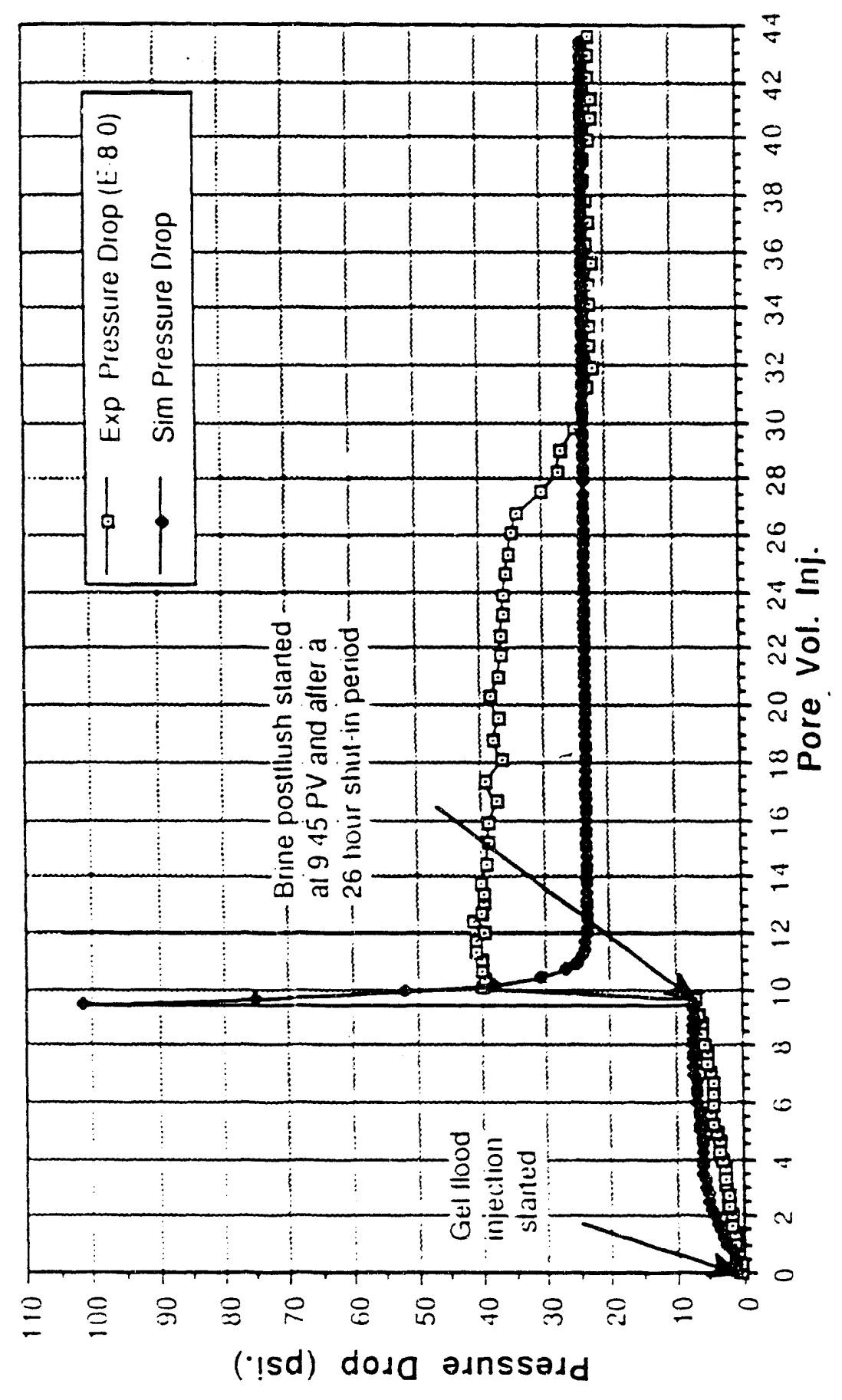

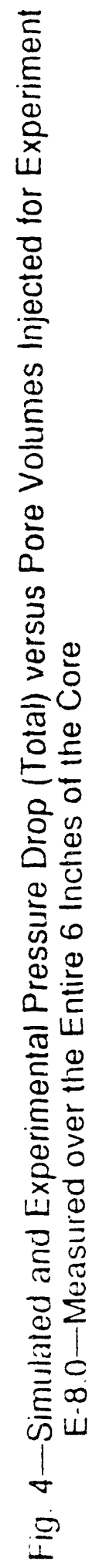


Subtask 2c - Simulation of Hydrodynamic Instabilities (Viscous Fingering) in Permeable Media

\section{Introduction}

Fine-mesh simulations of first-contact miscible displacements have been performed using UTCHEM. The results match the production history of a laboratory-scale core flood. An empirical viscous fingering model has also been implemented and tested. The model can successfully match the recovery curve of a first-contact miscible linear unstable displacement. Better results can be obtained by adjusting the viscosity mixing parameter.

A major difficulty of numerical simulation is the accurate modelling of the physical instability that occurs when a fluid is displaced by a more mobile fluid, a situation that is frequently present in both miscible and chemical displacement processes. Under most reservoir circumstances this instability or viscous fingering results in the bypassing of a substantial amount of target oil or inefficiently driving a slug.

One approach to modelling unstable flow is to perform fine-mesh simulation in which fingers are explicitly simulated. Several authors have used this approach to match laboratory-scale experiments (Araktingi and Orr, 1988; Christie and Bonr. 1987; Christie, 1987; Giordano et al., 1985). However, the number of grid blocks needed to perform such fine-mesh simulations on a field scale makes this approach impractical for reservoirscale simulation with present computing capabilities.

An alternative method for modelling unstable flow is to use an empirical viscous fingering model with a coarse mesh or one-dimensional grid structure, depending on the displacement geometry. These models are designed to match effluent histories and include viscosity modification approaches (Koval, 1963; Todd and Longstaff, 1972), fingering width approaches (Fayers. 1988; Fayers and Newley, 1988), and effective dispersion models (Young, 1986). The value of these models is a function of both their ability to predict displarement efficiency and their savings of computer time.

The objective of this study is to perform fine-mesh simulations using UTCHEM (Datta Gupta et al., 1988; Saad et al.. 1989; Saad, 1989), and then to either implement an existing model, modify an existing model, or develop a new model to represent unstable flows with a coarse mesh. The fine-mesh simulations are performed initially to test the simulator's ability to explicitly simulate displacements characterized by viscous fingering. Then the fine-mesh simulations will be used along with the work of other authors to determine which physical effects (viscosity relationships, non-Newtonian effects, 
heterogeneity, etc.) are the most important considerations in developing an empirical model. Finally, the model that is chosen, modified, or developed will be tested against. fine-mesh simulations and experimental data.

\section{Eine-mesh simulation of first-contact miscible displacement}

Description of Problem

A fine-mesh simulation of one experiment of Blackwell et al. (1959) was performed in order to test the simulator's ability to explicitly simulate an unstable displacement with first-contact miscible fluids. This and all successive simulations were run on the CRAY X-MP using UTCHEM version 3.43.

The experiment was carried out in an unconsolidated Ottawa sand pack that was six feet long, two feet wide and 3/8 inch thick. Injection was along the entire injection face of the sand pack and production was along the entire outlet end. The mobility ratio of the displac fluid to the injected fluid was 86 .

A $100 \times 72 \times 1$ grid was used in the simulation. The simulated flood consisted of water with a viscosity of $1 \mathrm{cp}$ displacing polymer with a peak viscosity of $86 \mathrm{cp}$. The polymer only increased the aqueous phase viscosity; it had no shear thinning, adsorption, permeability reduction, etc. A quarter-power viscosity rule was used such that

$$
\frac{1}{\mu_{\mathrm{aq}}^{1 / 4}}=\frac{1-C_{D 4}}{\left(\mu_{\mathrm{w}}\right)^{1 / 4}}+\frac{C_{D 4}}{\left(\mu_{\mathrm{p}}^{0}\right)^{1 / 4}}
$$

or

$$
\mu_{\mathrm{aq}}=\frac{\mu_{\mathrm{p}}^{\mathrm{o}}}{\left[\mathrm{C}_{\mathrm{D} 4}+\left(1-\mathrm{C}_{\mathrm{D} 4}\right) M^{1 / 4}\right]^{4}}
$$

Fingers were initiated by assigning uncorrelated normally distributed random permeabilities to each grid block. The standard deviation svas $5 \%$ of the average permeability. The pertinent input data used are listed in Table 1.

Results of Fine-Mesh Simulation

The results of the fine-mesh simulation reasonably match the recovery data and breakthrough time reported by Blackwell (1959). Figure 1 shows the contours of the fingers at $0.2 \mathrm{PV}$, and Fig. 2 shows the cumulative production as a function of pore volumes injected. As with the experiment, breakthrough occurred at 0.22 pore volumes of 
fluid injected. The cumulative recovery recovery curve overlays the Blackwell results almost perfectly until about 0.6 pore volumes injected. After $0.6 \mathrm{P}$.V. injected, the finemesh simulation results predict a recovery efficiency that is lower than the actual reported recovery efficiency.

The fine-mesh simulation took $1703 \mathrm{CPU}$ seconds to run.

\section{Use of Todd-Longstaff (1972) model to match a first-contact miscible displacement}

Description of Model and Input

The Todd-Longstaff viscous fingering model was implemented in UTCHEM and was tested against Blackwell's data. The formulation used was a pseudo-miscible displacement in which water ( representing "solvent") displacing oil was used to mimic a miscible displacement. The "miscibility" was accomplished by setting the capillary pressure equal to zero and using straight-line relative permeability curves. According to the Todd-Longstaff model, viscosities are replaced by the following effective viscosities:

$$
\begin{aligned}
& \mu_{\infty e}=\mu_{0}^{1-\omega} \mu_{m}^{\omega} \\
& \mu_{s e}=\mu_{s}^{1-\omega} \mu_{m}^{\omega}
\end{aligned}
$$

The mixture viscosity followed the quarter-power viscosity mixing rule (Eq. 1).

\section{Results}

Runs were made to simulate Blackwell's experiment using the Todd-Longstaff formulation with a $50 \times 1 \times 1$ one-dimensional grid. Figure 3 shows that using Todd and Longstaff's suggested value of $\omega=2 / 3$ gave a fair match for mobility ratios of 86 and 150 , a somewhat worse match for a mobility ratio of 5 and a rather high predicted recovery for a mobility ratic of 375 (note that Todd and Longstaff did not report results for a mobility ratio of 375 ).

\section{Conclusions}

We have shown that UTCHEM is capable of accurately performing fine-mesh simulations of first-contact miscible displacements that are characterized by viscous fingering. The simulator results satisfactorily matched a core-flood experiment performed 
by Blackwell (1959). The study further showed that the Todd-Longstaff viscous fingering model as implemented in UTCHEM can match the production history for a linear unstable first-contact miscible displacement . Better matches can be obtained by adjusting the viscosity mixing parameter.

\section{Euture work}

Our studies of current viscous fingering models will continue. The models which will be studied include, but are not limited to, the Fayers model (Fayers, 1988; Fayers and Newley, 1988), effective dispersion models (Young, 1986), a "transfer" model (Nghiem et al., 1989), and an effective relative permeahility model (Oteh, 1987: Peters and Khataniar. 1987). Some of the primary considerations that will go into choosing or developing a model are its ability to match experimental data and fine-mesh simulations, the degree to which the parameters are physically interpretable, the ability to determine parameters $a$ priori, whether or not multi-phase flow can be modelled and whether the model can adequately include physical properties of fluid flow such as non-Newtonian effects, dispersion, adsorption, etc.

Fine-mesh simulation of unstable displacements will continue in order to have a basis for testing empirical models. These simulations will also allow us to use the results in conjunction with the work of other authors to determine the relative importance of including the various physical properties in a viscous fingering model.

\section{References}

Araktingi, U. and F.M. Orr: "Viscous Fingering in Heterogeneous Porous Media," paper SPE 18095 presented at the 63rd Annual Technical Conference and Exhibition of SPE, Houston, TX, Oct. 2-5, 1988.

Blackwell, R.J., J.R. Rayne, and W.M. Terry,: "Factors Influencing the Efficiency of Miscible Displacement," Trans. AIME (1959) 216, 1-8.

Christie, M.A.: "Numerical Techniques for High-Resolution Simulation of Instabilities," paper SPE 16005 presented at the Ninth SPE Symposium on Reservoir Simulation, San Antonio, TX, Feb. 1-4, 1987.

Christie, M.A. and D.J. Bond: "Detailed Simulation of Unstable Processes in Miscible Flooding," SPE Res. Eng. (Nov. 1987), 514-522.

Datta Gupta, A., G.A. Pope, K. Sepehrnoori, and R.L. Thrasher: "A Symmetric, Positive Definite Formulation of a Three-Dimensional Micellar/Polymer Simulator," SPF: Res. Eng. (Nov. 1988), 622-632. 
Fayers, F.J.: "An Approximate Model With Physically Interpretable Parameters for Representing Miscible Viscous Fingering,"SPE Res. Eng. (May 1988), 551-558.

Fayers, F.J. and T.M.J. Newley: "Detailed Validation of an Empirical Model for Fingering With Gravity Effects," SPE Res. Eng. (May 1988), 542-550.

Giordano, R.M., S.J. Salter, and K.K. Mohanty: "The Effects of Permeability Variations on Flow in Porous Media," paper SPE 14365 presented at the 60th Annual Technical Conference and Exhibition of SPE, Las Vegas, NV, Sept. 22-25, 1985.

Koval, E.C.: "A Method for Predicting the Performance of Unstable Miscible Displacement in Heterogeneous Media," Soc. Pet. Eng. J. (March 1963), 28-40.

Nghiem, L.X., Y.K. Li, and R.K. Agarwal: "A Method for Modelling Incomplete Mixing in Compositional Simulation of Unstable Displacements," paper SPE 18439 presented at the Reservoir Simulation Symposium in Houston, TX, Feb. 6-8, 1989.

Odeh, A.S.: "A Proposed Technique for Simulation of Viscous Fingering in OneDimensional Immiscible Flow," paper SPE 17087 (1987).

Peters, E.J. and S. Khataniar: "The Effect of Instability On Relative Permeability Curves Obtained by the Dynamic-Displacement Method," SPE Form. Eval. (Dec. 1987), 469-474.

Saad, N.: "Field Scale Simulation of Chemical Flooding," Ph.D. dissertation, U. of Texas, Austin, Aug., 1989.

Saad, N., G.A. Pope, and K. Sepehrnoori: "Application of Higher-Order Methods in Compositional Simulation," to appear in SPE Res. Eng. (Nov. 1990).

Todd, M.R. and W.J. Longstaff: "The Development, Testing, and Application of a Numerical Simulator for Predicting Miscible Flood Performance," J. Pet. Tech., (July 1972), 874-882.

Young, L.C.: "The Use of Dispersion Relationships to Model Aciverse Mobility Ratio Miscible Displacements." paper "PE/DOE 14899 presented at the SPE/DOE Fifth Symposium on Enhanced Oil Recovery, Tulsa, OK, April 20-23, 1986. 


\section{Nomenclature}

$$
\begin{aligned}
& C_{D 4}=\frac{\text { polymer concentration }}{\text { initial polymer concentration }} \\
& M \quad=\text { mobility ratio }
\end{aligned}
$$

\section{Greek Symbols}

$$
\begin{aligned}
& \mu_{\mathrm{aq}}=\text { aqueous phase viscosity (cp) } \\
& \mu_{\mathrm{m}} \quad=\text { mixture viscosity (cp) } \\
& \mu_{0}=\text { oil viscosity (cp) } \\
& \mu_{\infty e}=\text { effective oil viscosity (cp) } \\
& \mu_{\mathrm{p}}^{0}=\text { polymer viscosity (cp) at } C_{D 4}=1 \\
& \mu_{\mathrm{s}} \quad=\text { "solvent" viscosity (cp) } \\
& \mu_{\text {se }} \quad=\text { effective "solvent" viscosity (cp) } \\
& \mu_{\mathrm{w}} \quad=\text { pure water viscosity (cp) } \\
& \omega=\text { Todd-Longstaff mixing parameter }
\end{aligned}
$$


Table 1. Input for Simulating Blackwell's Experiment

$\begin{array}{lll}\text { Simulated Area } & =6 \mathrm{ft} \times 2 \mathrm{ft} \\ \text { Thickness } & =0.03125 \mathrm{ft}(3 / 3 \text { inch }) \\ \text { Porosity } & =0.33 \\ \text { Average Permeability } & =200 \text { darcies } \\ \text { Permeability Distribution } & =\text { random: uncorrelated, normally distributed } \\ \text { Permeability Standard Deviarion } & =10 \text { darcies }(5 \% \text { of average }) \\ \text { Longirudinal Dispersivity } & =0.012 \mathrm{ft}(0.366 \mathrm{~cm}) \\ \text { Transverse Dispersivity } & =0.004 \mathrm{ft}(0.0122 \mathrm{~cm}) \\ \text { Molecular Diffusion Coefficient } & =0.00233 \mathrm{ft}^{2} / \text { day }\left(2.5 \times 10^{-5} \mathrm{~cm}^{-} ; \mathrm{sec}\right) \\ \text { Injected Fluid Viscosity } & =1.0 \mathrm{cp} \\ \text { Displaced Fluid Viscosity } & =86.0 \mathrm{cp} \\ \text { Injection Rate } & =0.825 \mathrm{ft}^{3} / \text { day } \\ \text { Numerical Grids } & =100 \times 1 \times 72 \\ \text { Cell Peclet Number }\left(\frac{\Delta x}{\alpha_{L}}\right) & =5 \\ \text { Maximum Time Step } & =0.0001875 \text { day }\end{array}$




\section{List of Figures}

Figure

1

Concentration contours from fine-mesh simulation of Blackwell's experiment at 0.20 pore volumes injecterd (concentrations shown are $\mathrm{C}_{\mathrm{D} 4}=0.1,0.5$, and $0.9)$

2 Comparison of Blackwell et al. (1959) recovery data with predicted recovery using UTCHEM for a mobility ratio of 86

3 Comparison of Blackwell et al. (1959) recovery data with predicted recovery using the Todd-Longstaff model in UTCHEM for $\omega=\frac{2}{3}$ 


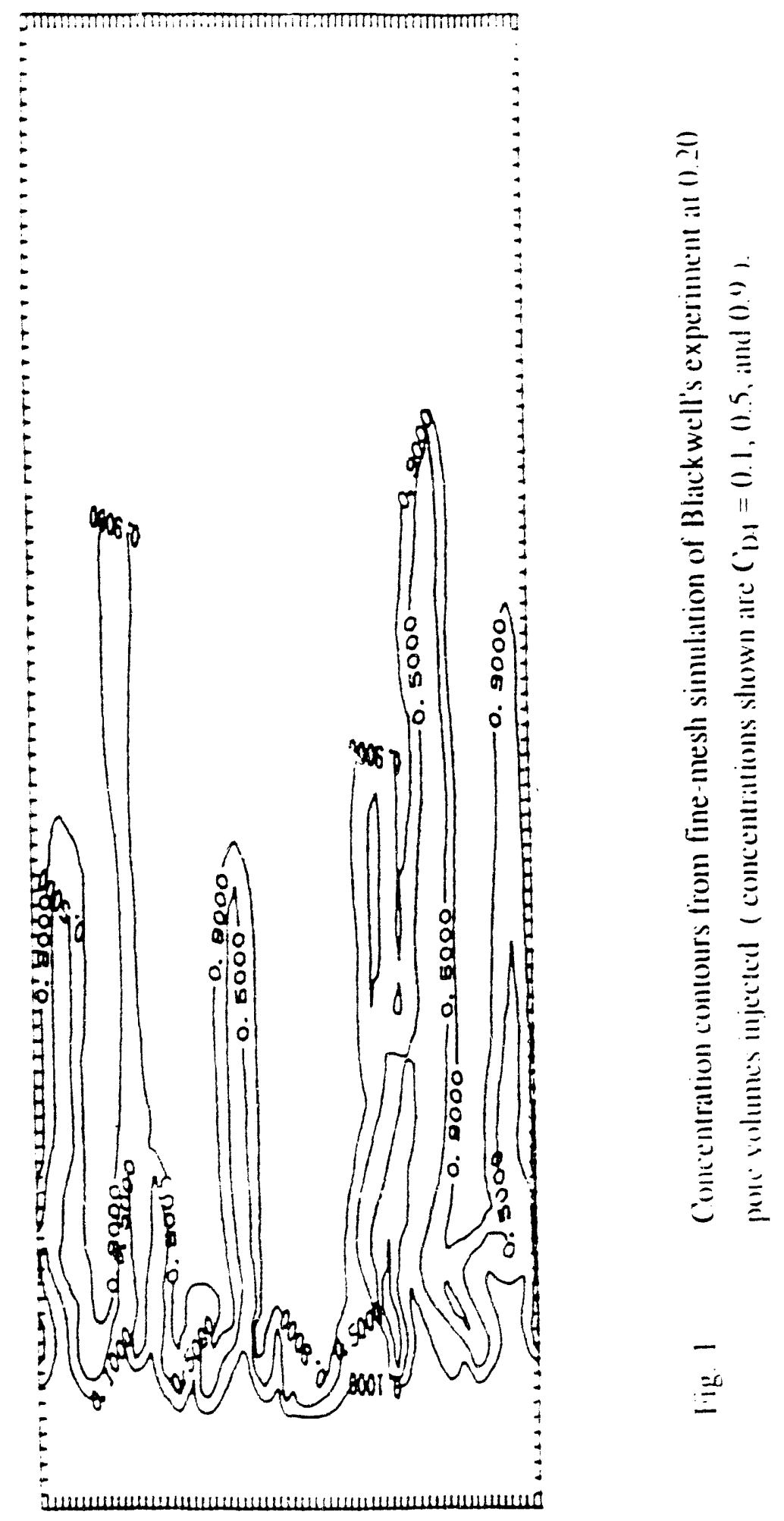




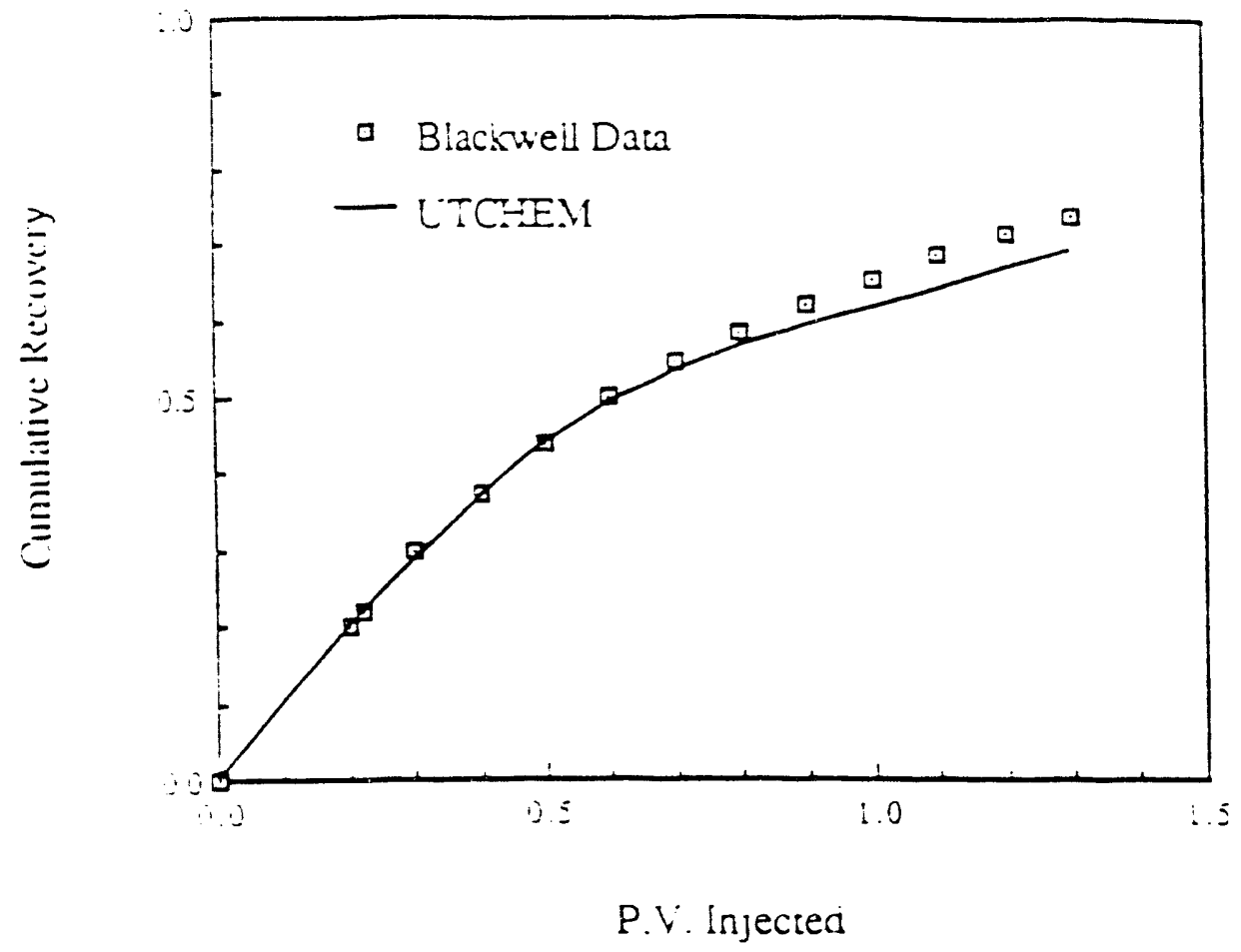

Fig. 2 Comparison of Blackwell et al. (1959) recovery data with predicted recovery using UTCHEM for a mobility ratio of 86 .

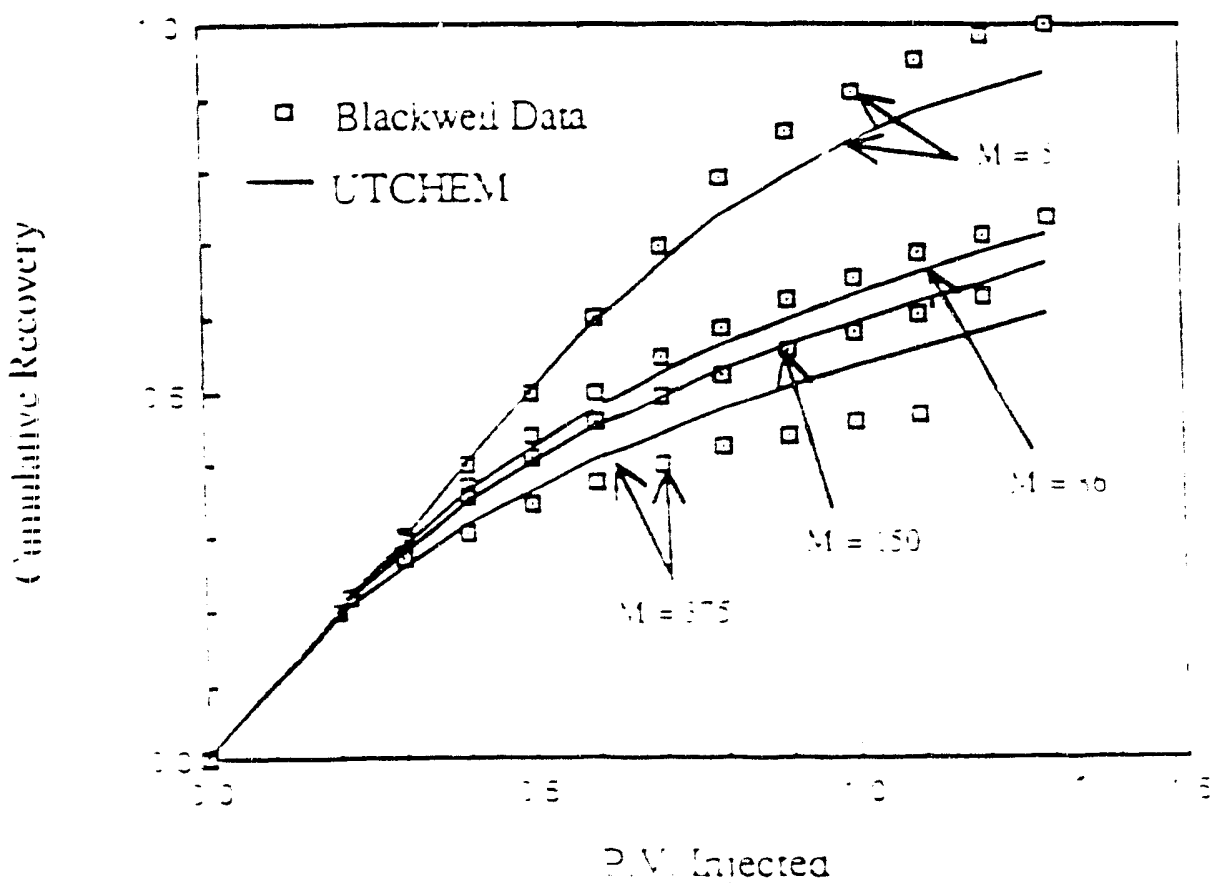

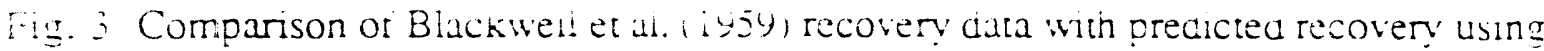
:he Todd-Longstatif model in LTCHE.M for $\omega=\overline{\bar{z}}$ 
Subtask 2d - Development of an "Expert System" to Design Chenical Floods Using a Compositional Reservoir Simulator

\section{Summary}

Computer simulators allow state of the art reservoir analysis in the petroleum industry, but their use can be cumbersome. The generation of input files can be an unwieldy task made more difficult by a lack of data or technique. The simulator output may consist of reams of computer paper from which the user wants a handful of values. This report describes a rule-based computer program designed to assist the user both in creating the input for and analyzing the output from a reservoir simulation. The user directs the program to complete these tasks by picking items from descriptive menus offered by the program. The program makes extensive use of computer graphics to show the user the effects of varying input values before running the simulation and to plot the results of the simulation so they may be easily interpreted. The program is implemented for use with UTCHEM, the Center for Petroleum and Geosystems Engineering (CPGE) chemical flooding simulator, but the technique used to write the program is general enough to allow it to be applied 10 other simulators with little effort. Future work will combine the program with a relational database and extensively test the system.

\section{Introduction}

Simulators are powerful tools for analyzing the performance of petroleum reservoirs, but they also can be complicated tools. To successfully use a simulator, a user must know not only the characteristics of the reservoir, but those of every reservoir fluid. For a chemical flooding simulator such as UTCHEM, this may require more than a hundred input parameters, including common ones, such as pcrosity and permeability, as well as those which are less common and for which data may not be readily available, such as cation exchange coefficients. Even when such data are available, they may be difficult to analyze or put in the form required for input into the simulator. Often no data exist under the exact conditions of interest, but do exist under similar conditions. This requires even greater knowledge of the process, property data, and property models used in the simulator and even more time to effectively and correctly use the simulator to its maximum extent. Often a suitable judgment of the adequacy of the input can only be made after trial simulations have been made, producing reams of output. The solution is computer assistance to interactively create and analyze input and output data. This project takes the 
first step towards that solution by providing the user with a computer interface to interactively create and analyze input parameters and graphically display the simulated results.

The first section of this report describes the hardware and software required for the project and the methodology used to build an expert system for reservoir simulation. The second section describes the specific implementation of this expert system for use with UTCHEM. The final section describes future plans to enhance this expert system.

\section{Project description}

The interface program (or interface) is written in FORTRAN and OPS5, an artificial intelligencie language supported by DEC. A ruie-based language was necessary for program efficiency because the user interacts with the program using menus, and menu systems naturally follow a branching structure well suited for rule-based languages. OPS5 was chosen over other rule-based languages, such as LISP, because of its speed and lack of structure. Due to its rule firing strategy, OPS5 programs are much quicker than LISP programs. In addition, the language is totally unstructured, meaning that rules are not executed in a preset order. Rules are written with condition and action elements. The program executes the action element only when it finds an exact match for the condition element in the program's working memory. The action elements create the menus and plots using DI-3000, a commercial graphics software package. DI-3000 is a relatively inexpensive, FORTRAN-based graphics package that allows a programmer :o create twoor three-dimensional images on a computer workstation. It is available for most popular workstations, including the department's DEC GPX. Since DI-3000 is FORTRAN-based, some FORTRAN programming is necessary to link OPS5 and DI-3000. The connection between FORTRAN and OPS5 is well supported by DEC. Finally, the interface may use a relational data base to store and retrieve reservoir and fluid data.

The specific aim of this project is to design an interface for UTCHEM, but an added benefit has been the creation of a generic interface that can be as easily applied to a black oil simulator or to UTCOMP, the CPGE's compositional simulator, as to UTCHEM. The heart of the interface is the menu-driven, user interaction component. Through menus the program guides the user through the process of setting input parameters and displaying the simulator output. The menus interact with graphics programs that show the reservoir and fluid properties and output results in graphs and contour plots. A third component is a database of previous simulation runs and typical parameter values that the user may use to reduce the ume creating an input file or to provide suggested values based on previous 
experience. The final coinponent, a knowledge base, includes parameter and plot characteristics which distinguish one implementation of the interface from another. These characteristics transparently provide information about a parameter or plot to the interface. It is this component that must be changed in order to apply the interface to different simulators. In addition, the knowledge base may be easily updated to reflect new features added to a simulator. Each of these components is explained in greater detail below.

The menu component allows four types of user interaction (called interactors): Command, Parameter, Grid, and Graphic. The Command interactor allows the user to pick one or more commands from a menu offered by the program. Each menu represents a decision point in the interface logic. By selecting a command, the user instructs the interface which branch to follow from the point. The Parameter interactor presents parameter menus which group similar reservoir and fluid properties and allow unlimited user interaction to set, reset, and inquire about values. The Grid interactor presents gridded arrays detailing the Reservoir Description, Reservoir Conditions, and Recurrent Data in a graphic representation of the gridded reservoir. It is similar to the Parameter interactor in that the user may interact with the grid repeatedly. The Graphic interactor allows the user to inquire what commands are available to interact with screen graphics displays, such as printing. Each of these interactors is linked through the knowledge base.

The graphics component is essential for the generation of all workstation images. This includes menus, grids, and plots. Menus present the user with a set of choices that allow branching in the program logic. Grids show a graphic representation of the userdefined grid and are used to display reservoir parameters and recurrent data. Plots are available both during the creation of input and the presentation of output. Input plots use values of input parameters to plot fluid properties, such as fluid viscosity and phase behavior, before the user runs the simulation. Output plots are generated for both histories and profiles. Histories may plot single- or multiple-component production. Contoured profiles are available for pressure and - oncentration distributions at any time during the simulation as well as for initial reservoir conditions.

The knowledge base contains menu, data, plot, and grid descriptions that are specific to each implementation of the simulator interface. Each of these descriptions is defined in a model. The Menu model describes the branch network for the menuing system. The Data model relates the rock and fluid parameters to one another. The Plot model describes characteristics of plots that may be generated by the program. The Grid model provides the descriptions necessary to draw a customized reservoir grid. 
The Menu model describes the information necessary to produce any type of menu using a set of menu attributes, such as a title and menu choices. The model provides for three types of menus: permanent, temporary, and multipick. Permanent menus do not disappear after the user chooses an item. The program allows the user to repeatedly choose from a group of parameters until the user decides to quit the menu. They are useful for parameter menus described as part of the Parameter interacto. Most branching is performed using temporary menus. They allow the user to choose a single command, such as "Set Value" for a parameter, and then disappear. Multipick menus are similar to temporary menus except that the user is allowed to choose multiple values which are grouped into a new menu.

The Data model consists of two linked elements which have associated with them a number of attributes to describe the simuidtor parameters. The most specific element describes the parameters themselves. The model recognizes two types of parameters based upon the "type" attribute, which associates a temporary menu of commands that may be selected to set values and query the knowledge base for information about a parameter. Most parameters are "typein" parameters and are associated with a single value. "Toggle" parameters have a list of values associated with them from which the user may choose. Values set by the user are checked to insure that they are reasonable and have acceptable units. The program converts the units to the unit system used by the simulator and writes the resulting values to an input file. In addition, these parameters have default values which may be used when the parameter is not important to the sirnulation but still needs a value in the input file.

The parameters are grouped by the properties they describe into classes. A class may be associated with graphs drawn using the parameter values from the class. These plots allow the user to examine the effects of varying parameter values and to compare them to plots of laboratory data. The user needs no knowledge of the underlying function necessary to generate a plot, only enough background to appreciate the changes caused by varying the values of the parameters. The user may go back and forth between the parameter menu and the plot window at any time. Classes describing a similar reservoir or fluid property are grouped into a superclass. Once the user has set values for all the parameters in the superclass, the program can generate plots displaying this property using these values. The user may not interact with these plots except to print a hardcopy but may return to the parameter menus, reset their values, and then generate the plots again.

The Plot model is composed of three elements. The elements have associated attributes which describe the data to be plotted, any functions that must be applied to the 
data before generating the plot, and the characteristics of the plot window. The user only needs to know the name of a desired plot, and the model provides the information to generate the plot.

The curve element describes the link to the Data model necessary to generate lines or curves on a plot. The name of a curve associates it with one or more graphs that plot that curve. The curve type specifies whether it is a straight line or a curve. The two attributes, $y$-intercept and slope, link the curve with the parameters from the Data model needed to generate a plot. When more than one parameter is needed to calculate a value for a curve attribute, the attribute points to a function. The function name matches the value in the curve attribute and indicates the parameters that are grouped to calculate the curve attribute. Each implementation of the interface requires that any needed functions be included with the knowledge base.

The axis element provides the information necessary to create a plot axis. Similar to the curve element, the name of the axis binds it to one or more graphs. Other attributes specify the grid type, linear or log, axis annotations, and endpoints. An axis element may be used for either the $\mathrm{x}$ or $\mathrm{y}$ axis.

The graph element describes the information necessary to create a plot window. The graph name links the plot to a menu choice. The user picks the name from a menu, and the program creates the plot using the knowledge base attributes. The title attribute provides a menu heading that may be different from the name. The axes are linked to the graph using the $x$-axis and $y$-axis attributes that contain the names of axis elements. The curves attribute links to one or more curve elements that appear in the plot.

The Grid model provides the descriptions rueeded to create different grids. The current version of the interface only provides for rectangular grids, so this model is not fully developed.

\section{Implementation with UTCHEM}

The following section describes a step-by-step example of using the interface implemented for UTCHEM to build an input file and examine the graphic output. At each step the user interaction is explained, and the resulting change in the workstation screen is shown in a figure.

After the system initializes itself, the user is presented with the "Main Menu" in Fig. 1. The user chooses to "Create Input" and is presented with a "Process .Options" menu. The user picks any item, "Surfactant Flood", for example, and the program creates a parameter menu for the "Component Names" (Fig. 2). It also expects the user to provide 
the number of alcohols and tracers for the simulation. The names are important for queries to the database, if it exists. If the program finds a fluid in the database with the same name as one used in the current simulation, it notifies the user that any parameter values associated with that fluid may be read from the database.

Once the user quits this menu, the system offers the "Input Options" menu from which the user builds the input file for the reservoir (Fig. 1). The first choice is "Title". The program stores the simulation title, the reservoir name, the user's name, and the date in the input file and in the database, if available. In addition, it searches the database for a entry with the simulation title or reservoir name. If one is found, the user may read the entire inp $\mathrm{st}$ file from the database and exit the program to run the simulation.

"Program Control" defines "Output Options" and "Job Options" (Fig. 3). The "Output Options" item groups "Print Options", "Print Times", "Plot Times", "History Components", and "Profile Components" (Figs. 4 through 6). The first three are parameter menus which allow the user to set the types of output desired and the times at which the program should print production history data. The latter two options are multipick menus that build new menus to be used by the user to generate output plots. From "Job Options" the user chooses "Setup Control", "Parameter Control", or "Time Step Control" (Figs. 7 through 9). The program presents a parameter menu which allows the user to set and reset values repeatedly. The "Time Step Control" parameters are initialized with reasonable values for the simulation process chosen above, but the user is free to modify them. The program warns the user if the modified values might cause an error in the simulator.

Choosing "Process Parameters" from the "Input Options" menu (Fig. 1) creates a menu of fluid properties. Choosing one of these properties in turn produces a menu of parameter classes that group parameters having similar characteristics that describe the property. In Fig. 10 the user chooses the "Relative Permeability" property and the "OilWater High IFT" class to create a menu of parameters. Picking "End Point Rel Perm Water" generates a menu of commands that set the value of the parameter or query the preset database, or knowledge base, for information about the parameter. The "Set Value" item asks the user to enter a value and units for the parameter (Fig. 10). The program converts the value to the computation units used by the simulator and checks the value against the knowledge base to insure that it is reasonable. If the value is too large or too small or if the program does not recognize the units, the program informs the user of the error, deletes the entry, and asks the user to enter another value. If not interested in the effects caused by a particular parameter, the user chooses the "Default Value" option, and 
the program inserts a value stored in the knowledge base. The "Help" option provides an more complete description of the parameter, and the "Units" option prints a list of units that may be used to enter the parameter value.

Once all the parameters are set, the user selects "Plot Property" from the menu, and the program creates a plot window using descriptions from the knowledge base (Fig. 11). Buttoning on the "pick" box next to the plot title generates another menu of commands for interaction with the plot window. The user first plots the property with the values set in the parameter menu. The resulting curves may be compared against laboratory data, if available. Since the curves in Fig. 11 differ significantly from the laboratory data, the user draws two new curves that better represent those data. By choosing the "Values From Curve" option, the user instructs the program to reset the parameter values based on these new curves. The "Remove Curve" feature allows the user to delete any curve. Once satisfied with the parameter values, the user quits from both the parameter menu and its associated plot.

After setting values for the other parameter classes using the same technique, the user selects "Property Plots" from the "Relative Permeability" menu (Fig. 10). The program displays a menu of possible plots that may be generated using all of the "Relative Permeability" parameters (Fig. 12). The user chooses "Residual Saturation", and the program displays the plot window showing residual saturation plotted against capillary number for all three phases. Buttoning on the "pick" box allows the user to print the plot or shrink it to an icon, a small rectangle displaying the title of the plot, as shown in Fig. 13. Now the user selects "Permeability Exponent" from the "Property Plots" menu, and the program draws a plot of the relative permeability exponent against capillary number (Fig. 13). After examining the plots, the user quits from each one, and the program returns to the "Relative Permeability" menu (Fig. 10). The user now may reenter any parameter menu to reset a parameter value or abort from the menu to the "Input Options" menu (Fig. 1).

Choosing either "Reservoir Description" or "Reservoir Conditions" from the "Input Options" menu (I.g. 1) allows the user to set gridded parameters. Il "Rescrvoir Description" option sets "Porosity", "Permeability, and "Elevation". The "Reservoir Conditions" option sets "Pressure", "Water Saturation", "Calcium Concentration", and

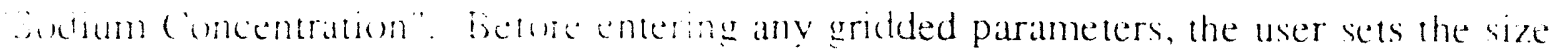
and dimensions of the grid in the "Grid Parameters" menu (Fig. 14). Once this menu is complete the program offers a menu of revervoir parameters. The user chomes "Porosity"

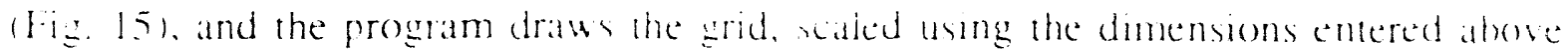




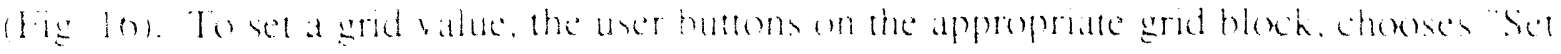
Valace", and enters a vake and units. The program prints a small circte an the center of the grid block and stores the value imemally Values may be set for multiple blacks by buttoning at the end of a column or row or by huternge on the "all" bleck. The value that is entered is effective for the entire column or row or the whole grid. Choosing "Show Value" returns the value sored for the grid block. If the grid blocks are too small wo reat. the user may bution on the "pick" box and choose "Zoom". The program zooms in on a user-chosen region, doubling its size. The user may instruct the program to contour the parameter values over the grid using the "Contour" option. Choosing "Done" returns the user to the "Reservoir Description" or "Reservoir Conditions" menu, and the process is repeated for other parameters. The user has the option to return to any grided parameter at any time to enter new values.

When the user chooses "Recurrent Data", the program draw's the reservoir grid with any existing wells. The user interacts with the grid by picking a block, and the program display's the "Well Options" menu (Fig. 17). The user chooses to "Add Well" and the program provides a menu of well types. The user selects a "Rate Dependent lniector". This causes the program to draw a well symbol and to display a parameter menu for the well specifications (Fig. 18). Buttoning on "Injection Rate" or "Injection Concentration" creates an additional menu of possible fluids to inject. The user must anstgn a value for each fluid in the menu. Locating a "Rate Dependent Producer" only requires a single ratle parameter. Pressure dependent wells require a single value for the hotom hole thowing pressure. The user also specifies the lavers in which the well is complend Piching the "Layers" parameter presents a multipick menu of the possible layers. When tinished entering the well characteristics. the user buttons on the "done" hox for the menu and picks another grid block. Choosing the "pick" box at the up of the grid returns liv ane: whe the "Input Options" menu. As with all the input options, the user may return to modify the "Recurrent Data" at any time during the input process

Once all the input parameters are set. the user abort we the Man Menu" and chooses "Run UTCHEM". The program asks for a file name and genterates an impun fike for the simulator. The user must "End" the program and sahmot the simulator ran hetore retuming to the program to examine the output

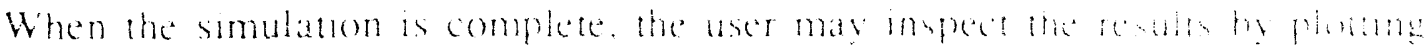

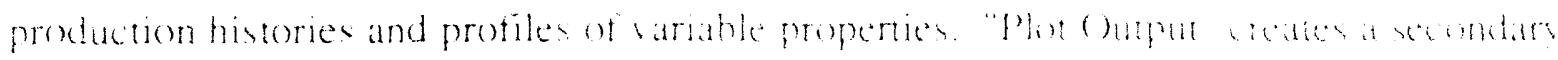

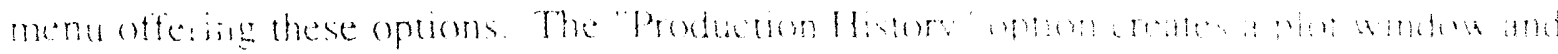

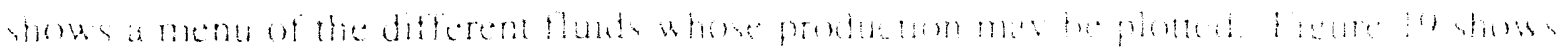




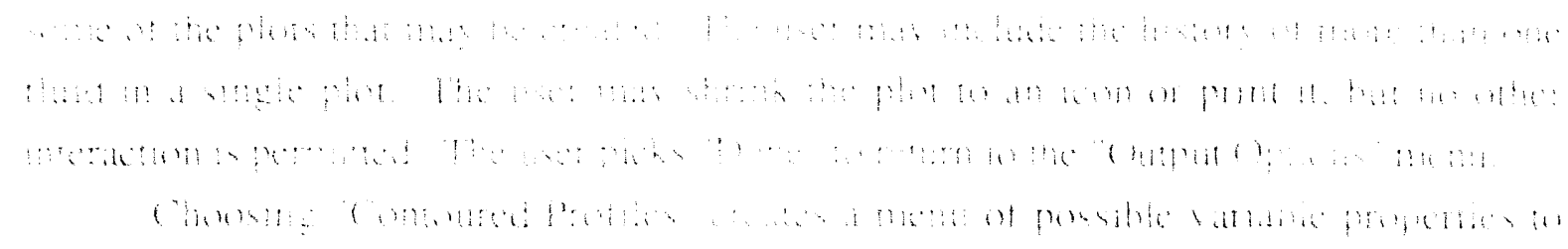

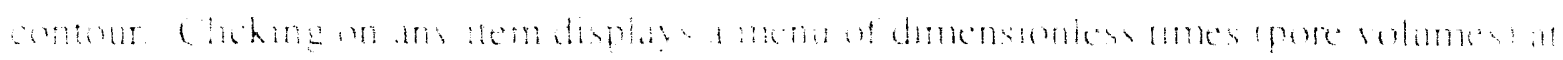

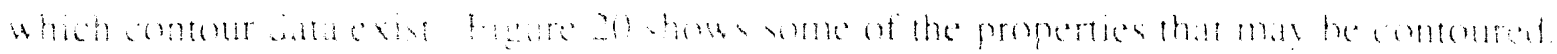

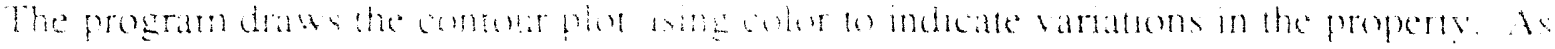

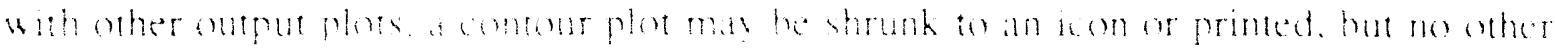

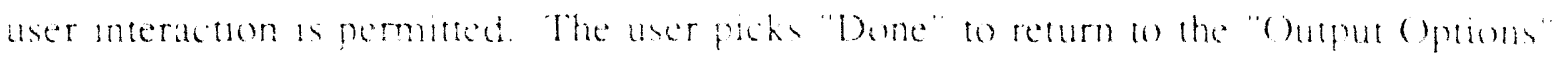

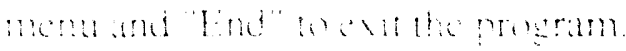

\section{Euture plans}

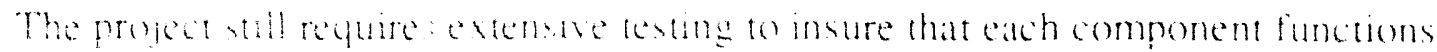
propery. In addion, a relational database will be tully integrated whthe other components o allow the user to store mput hites and parameter values for use in other simulation runs. 


\section{List of ligures}

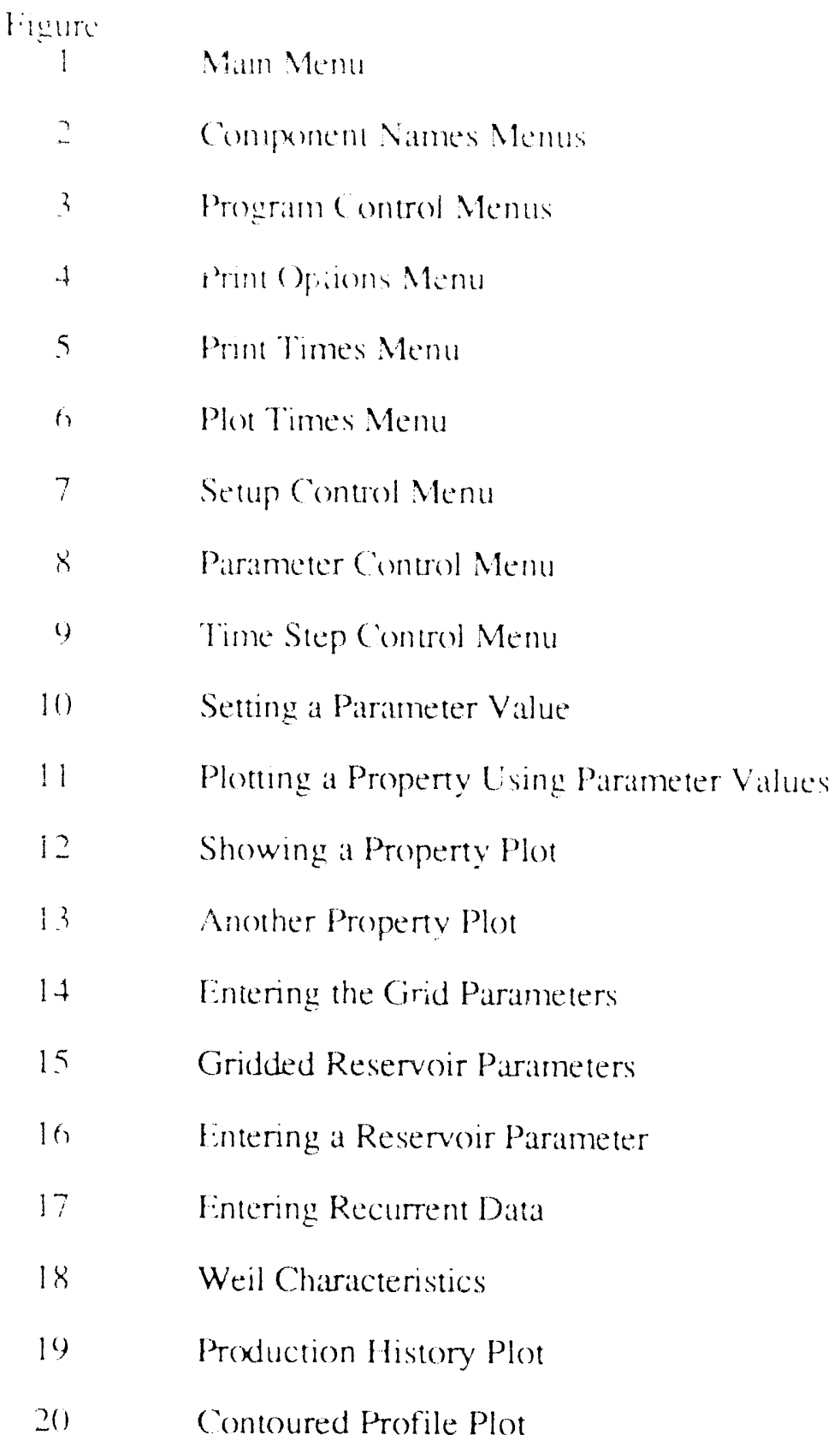




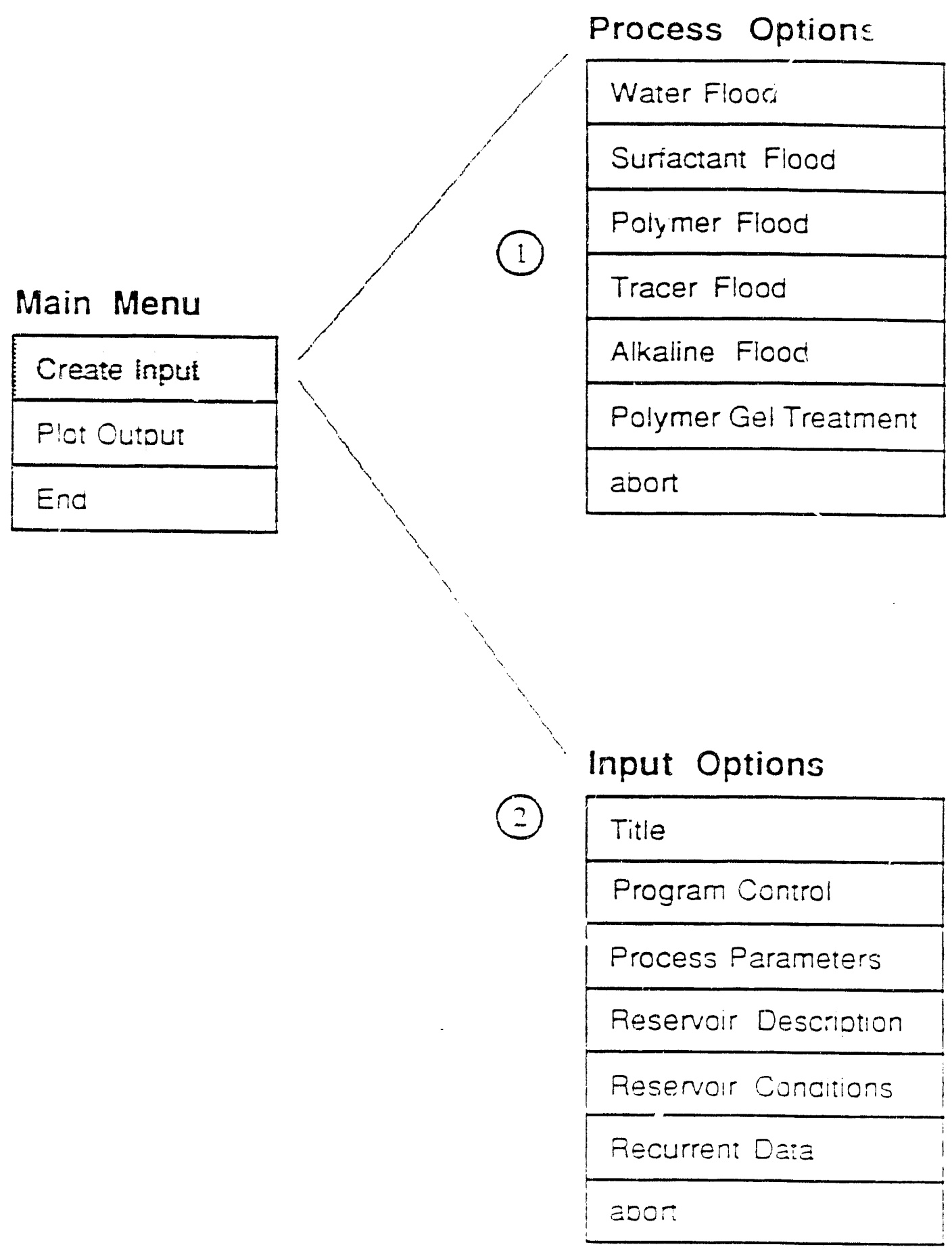

Figure 1

Main Menu 


\begin{tabular}{l}
\hline Cone Component Names \\
\begin{tabular}{|l|c|}
\hline Suriaciant & suri name \\
\hline Polymer & poly name \\
\hline Number of Alcohols & 1 \\
\hline Number ci Tracers & 2 \\
\hline
\end{tabular}
\end{tabular}

\section{done Alcohol Names}

\begin{tabular}{|l|l}
\hline Alcohol 1 & alc name \\
\hline
\end{tabular}

cone Tracer Names

\begin{tabular}{|l|l|}
\hline Tracer 1 & trac1 name \\
\hline Tracer 2 & trac2 name \\
\hline
\end{tabular}

Figure 2

comocnent Names ivenus 


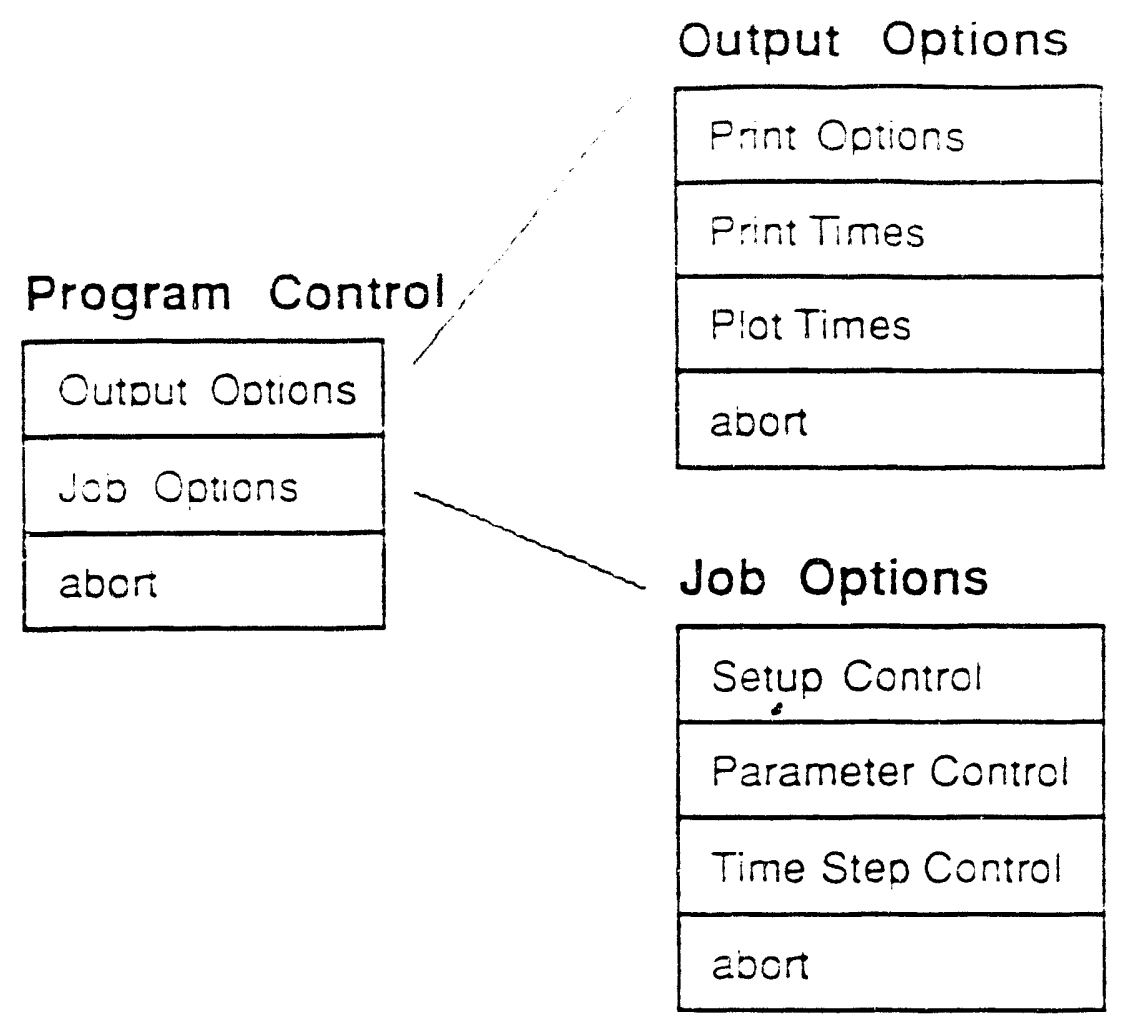

Figure 3

Program Control Menus

\section{Print Options}

\begin{tabular}{|c|c|}
\hline Priase Pressure & yes \\
\hline Acsorction Daia & ro \\
\hline Cuncerira:or & $y=5$ \\
\hline$K, Y, Z$ Phase Fiuxes & no \\
\hline Phase Viscosity & yes \\
\hline RElatwe Fermeasmy & yes \\
\hline Jasllary : Wmoer & 70 \\
\hline =erm Fecucion Facior & .0 \\
\hline
\end{tabular}

$$
\begin{aligned}
& \text { zare - } \\
& \text { Print Gouions ivenu } \\
& 127
\end{aligned}
$$


done Print Times

\begin{tabular}{|l|c|}
\hline Profile PV Interval & $0.1 \mathrm{PV}$ \\
\hline History PV Interval (unit 5) & $0.05 \mathrm{PV}$ \\
\hline History PV Interval init 6) & $0.0 \mathrm{PV}$ \\
\hline
\end{tabular}

Figure 5

Print Times Menu

cone Plot Times

\begin{tabular}{|l|l|}
\hline Profile PV Interval & $0.2 \mathrm{PV}$ \\
\hline Hision PV Interval & $0.1 \mathrm{PV}$ \\
\hline
\end{tabular}

Figure 6

Plot Times Menu 


\begin{tabular}{|l|c|}
\hline Fone Setup Control \\
\hline First Run or Restart & First iun \\
\hline Time Sizp Selection & Method 3 \\
\hline Solution Method & JCG \\
\hline rvumerical Dispersion Control & Leonard \\
\hline
\end{tabular}

Figure 7

Setup Control Menu

\begin{tabular}{l}
\hline oone Parameter Control \\
\begin{tabular}{|l|c|}
\hline Alconol Partitioning & Hirasaki \\
\hline Polymer Partitioning & yes \\
\hline
\end{tabular}
\end{tabular}

Figure 8

Parameter Control Menu

Cone Time Step Control

\begin{tabular}{|l|c|}
\hline Initial Time Sted & 0.01 Days \\
\hline Conc Change Tolerance & 0.001 \\
\hline Maximum Time Step & 0.05 Davs \\
\hline Minmum Time Step & 0.005 Davs \\
\hline Total Simulaied Time & 100.0 Davs \\
\hline
\end{tabular}

Figure a

Time Sted Control Menu 


\section{Fluid Properties}

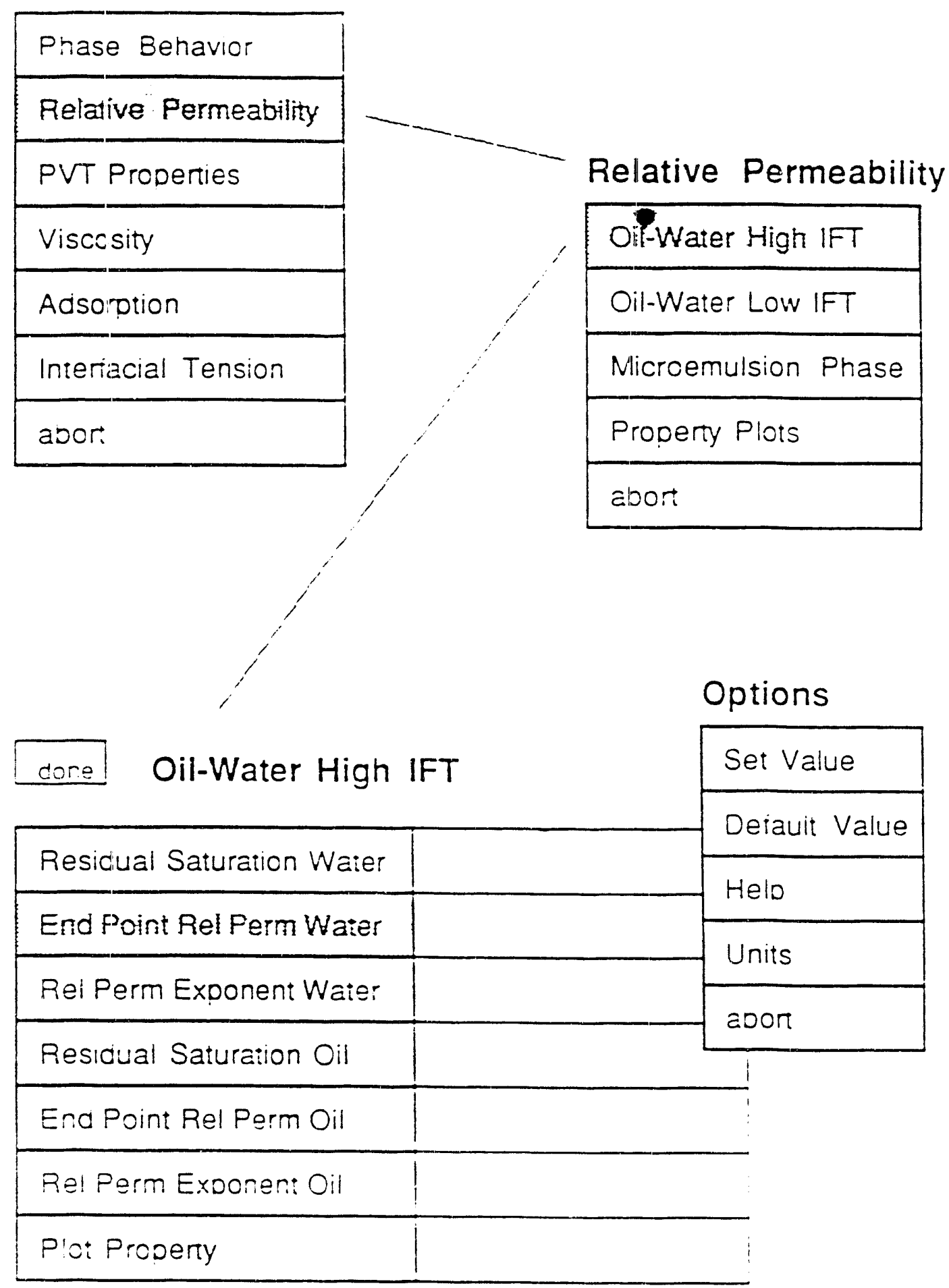

Figure 10

Serting a Parameter value 
pick Oil - Water Relative Permeability

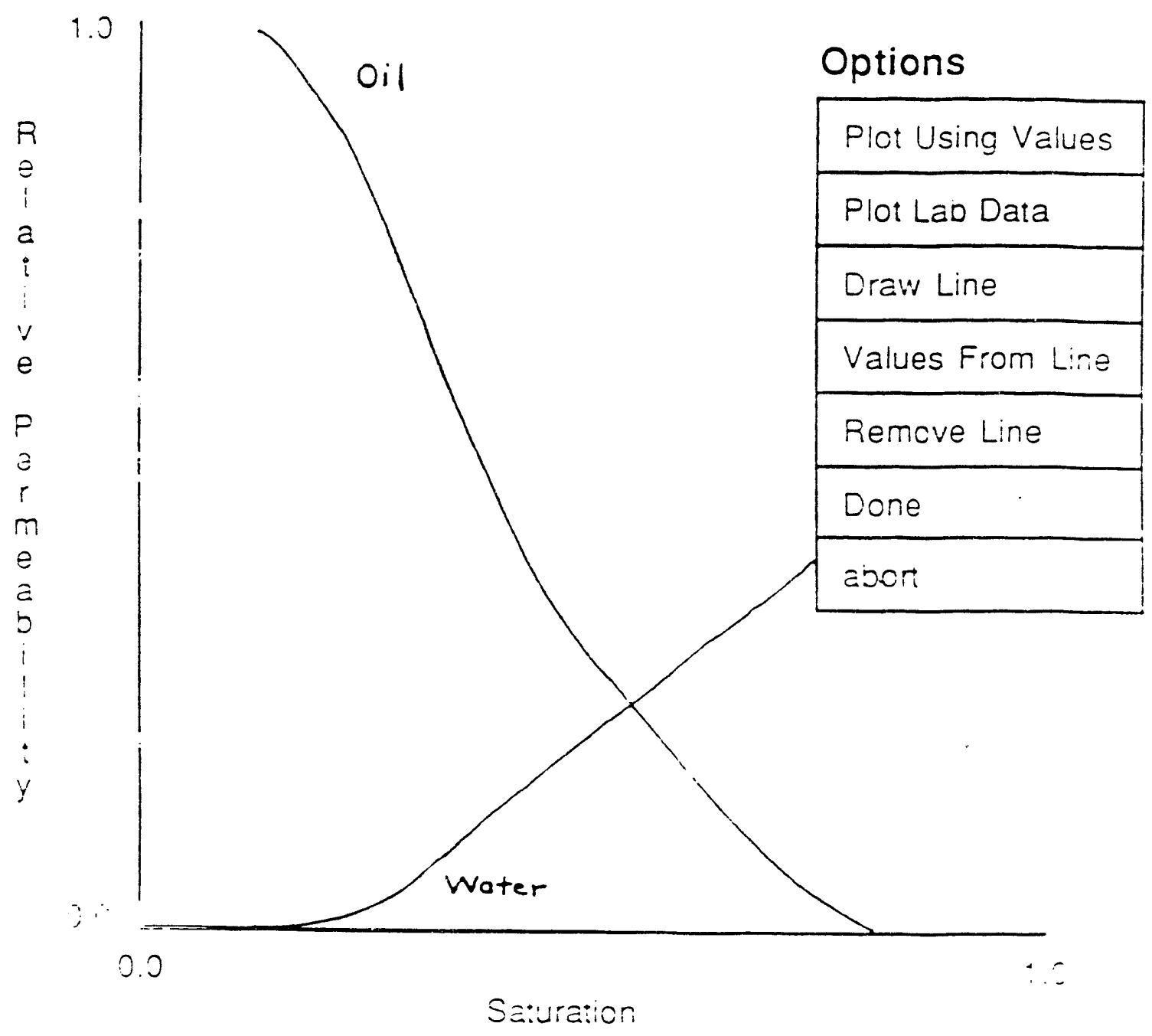

Figure :1

Ploming a Proveny Using Parameter Values 
Relative Permeability Plots

\begin{tabular}{|l|}
\hline Residual Saturation \\
\hline End Point Relative Perm \\
\hline Permeadiity Exponen: \\
\hline abort \\
\hline
\end{tabular}

pick Residual Saturation vs. Capillary Number

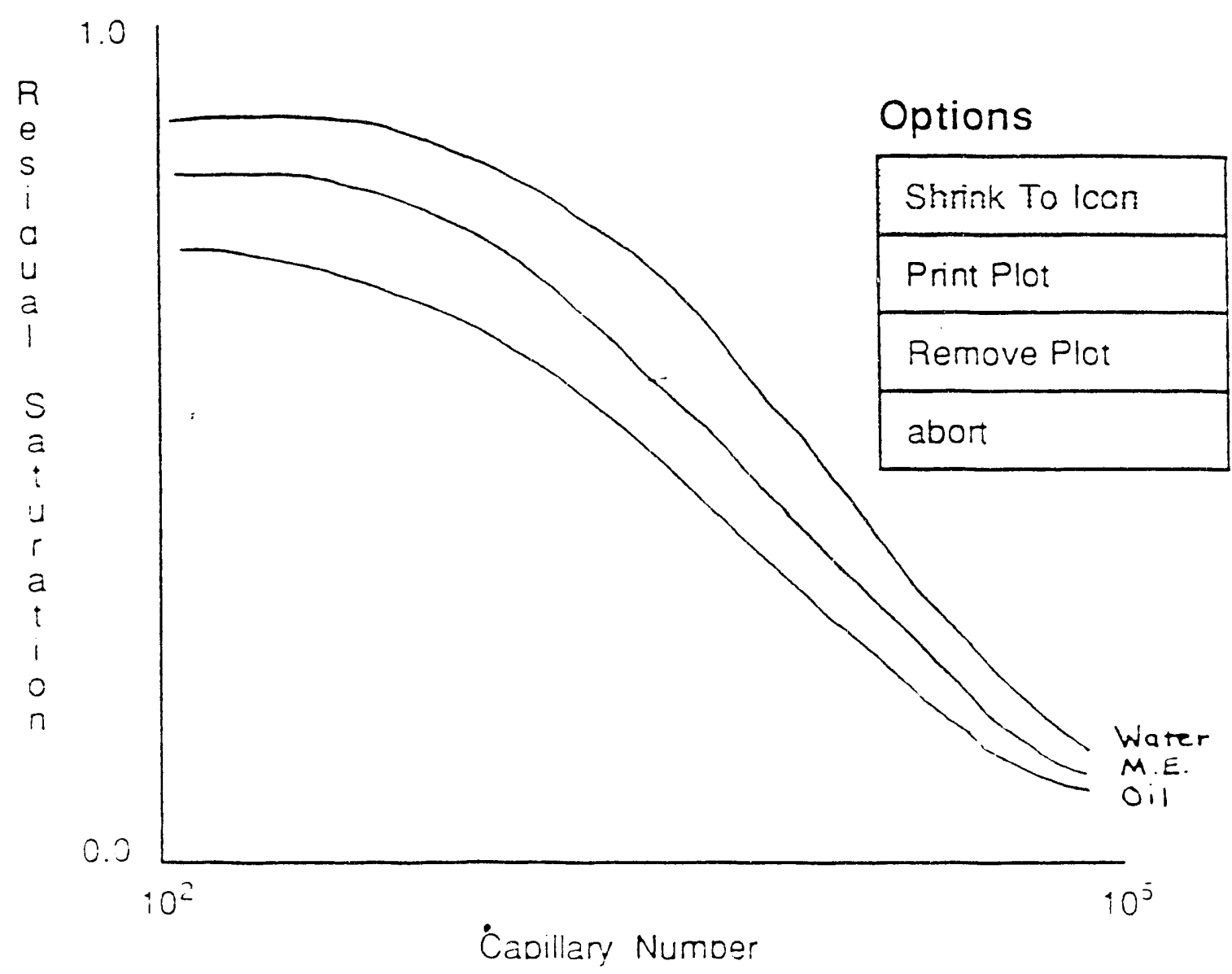

Figure 12

Sriowing à Propeny Plc: 


\section{Relative Permeability Plots}

\begin{tabular}{|l|}
\hline Residual Saturation \\
\hline End Point Relative Perm \\
\hline Permeability Exponent \\
\hline abor \\
\hline
\end{tabular}

Residual Saturation Icon

pick Permeability Exponent vs. Capillary Number

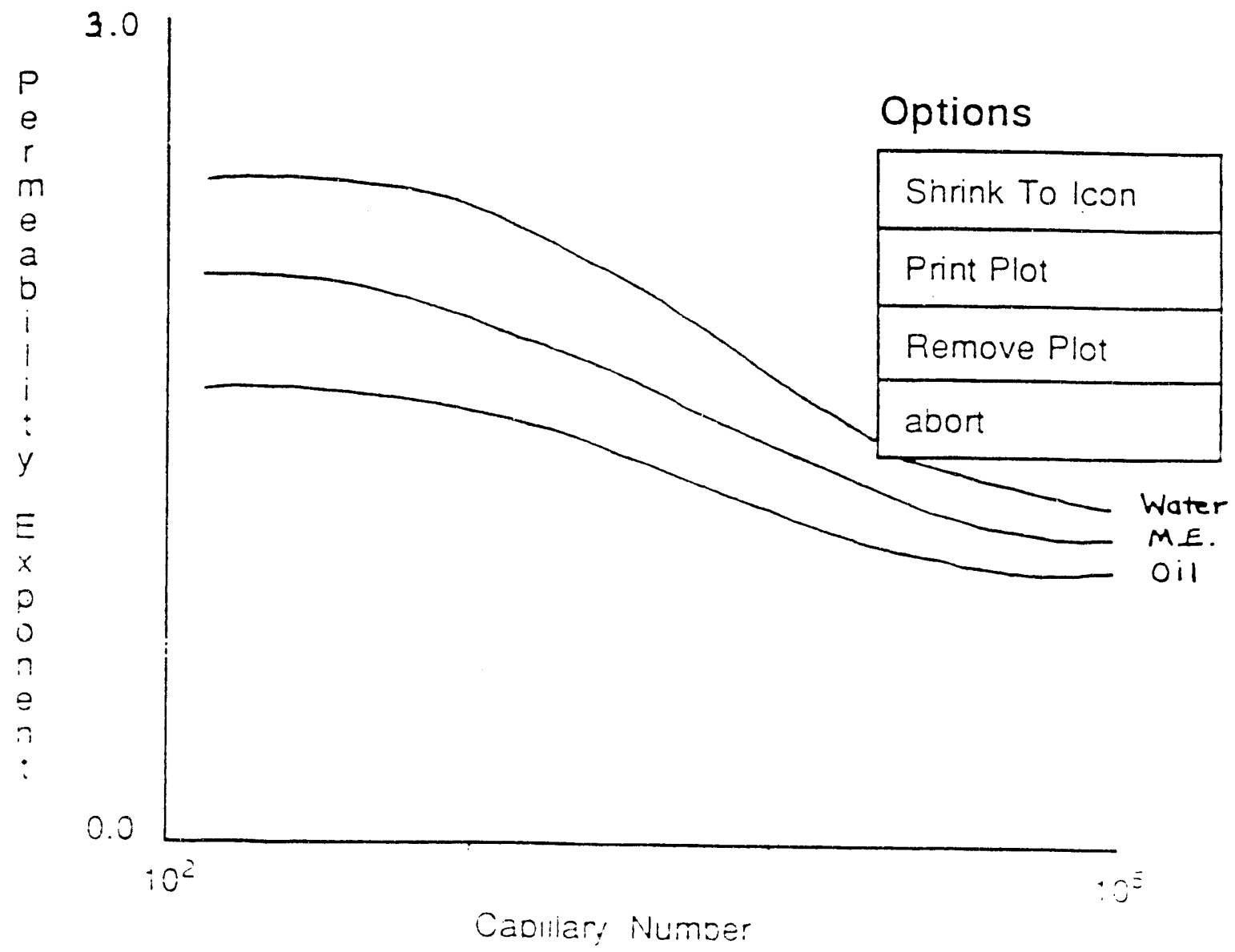

Ficure i3

Another Proderiy Plot 


Giid Parameters
\begin{tabular}{|l|}
\hline Grid Size \\
\hline$X$ Grid Dimensions \\
\hline Y Grid Dimensions \\
\hline Z Grid Dimensions \\
\hline Tctal Grid Dimens \\
\hline diluri \\
\hline
\end{tabular}
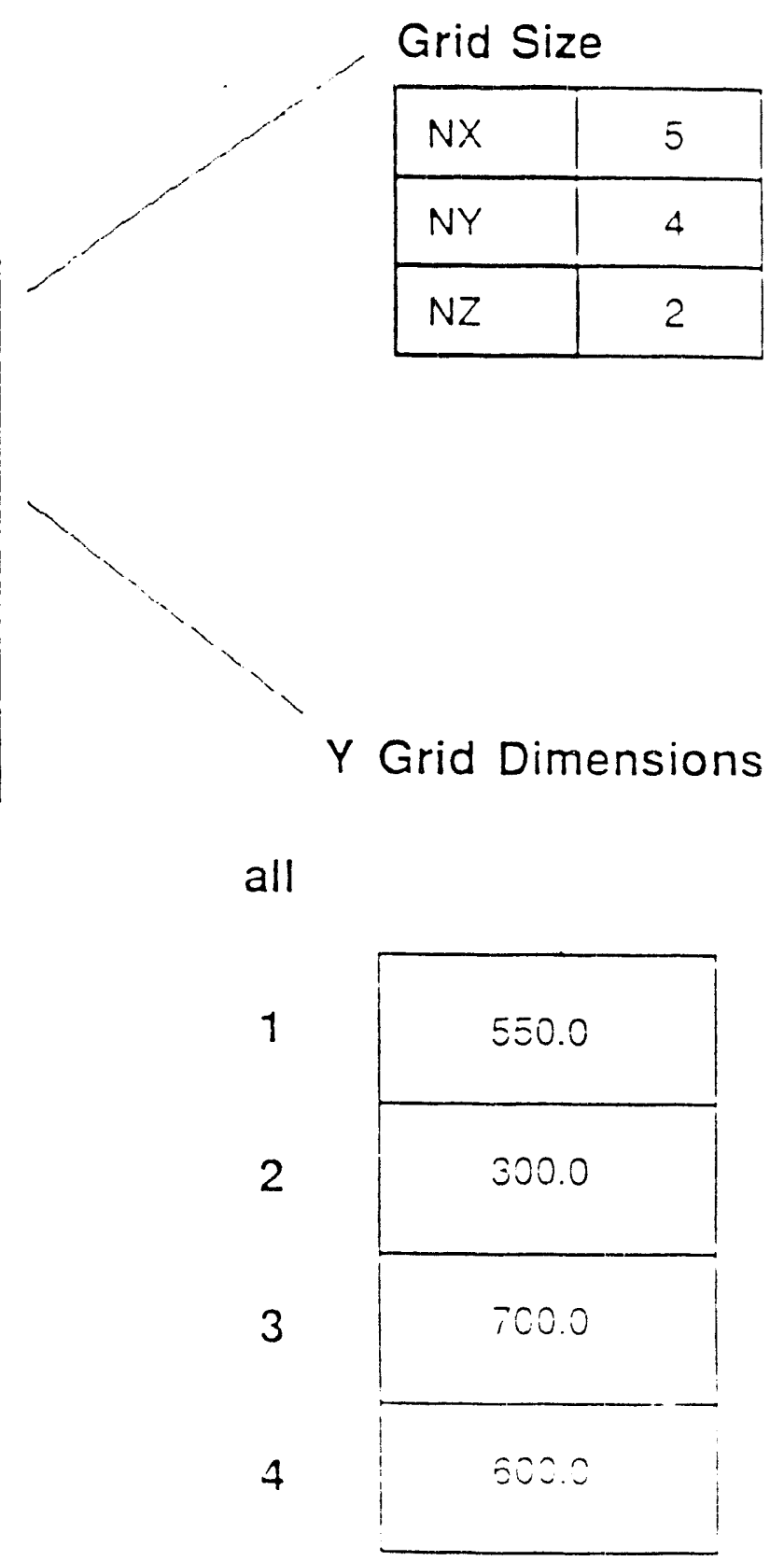

Figure $:$

Enterirg ine Grid Parameters 
Reservoir Description

Input Options
\begin{tabular}{|l|}
\hline Titie \\
\hline Program Con:rol \\
\hline Process Parameters \\
\hline Reservoir Description \\
\hline Reservoir Concitions \\
\hline Fecurren: Cata \\
\hline aoon \\
\hline
\end{tabular}

\begin{tabular}{|l|}
\hline Porosity \\
\hline Prmeavihty \\
\hline Eevations \\
\hline acor \\
\hline
\end{tabular}

Reservoii Conditions

\begin{tabular}{|c|}
\hline Fressure \\
\hline Water Saturation \\
\hline Calcium Concentravon \\
\hline Socium Conceriration \\
\hline acort \\
\hline
\end{tabular}




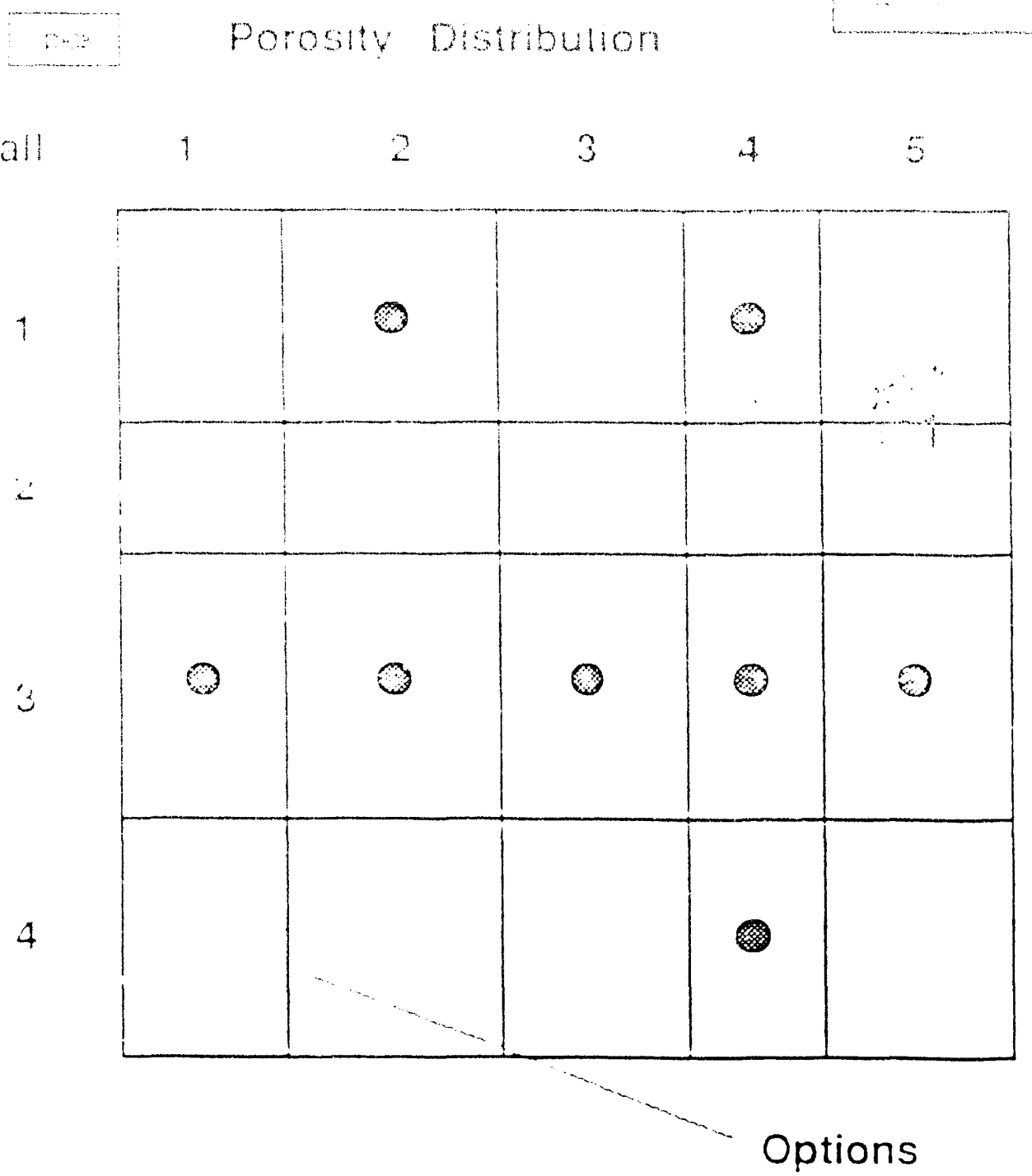

\begin{tabular}{|l|}
\hline Set Value \\
\hline Show Value \\
\hline abort \\
\hline
\end{tabular}

Figure 16

Enienng a Reservorr Parameter 
Fiecurront Data

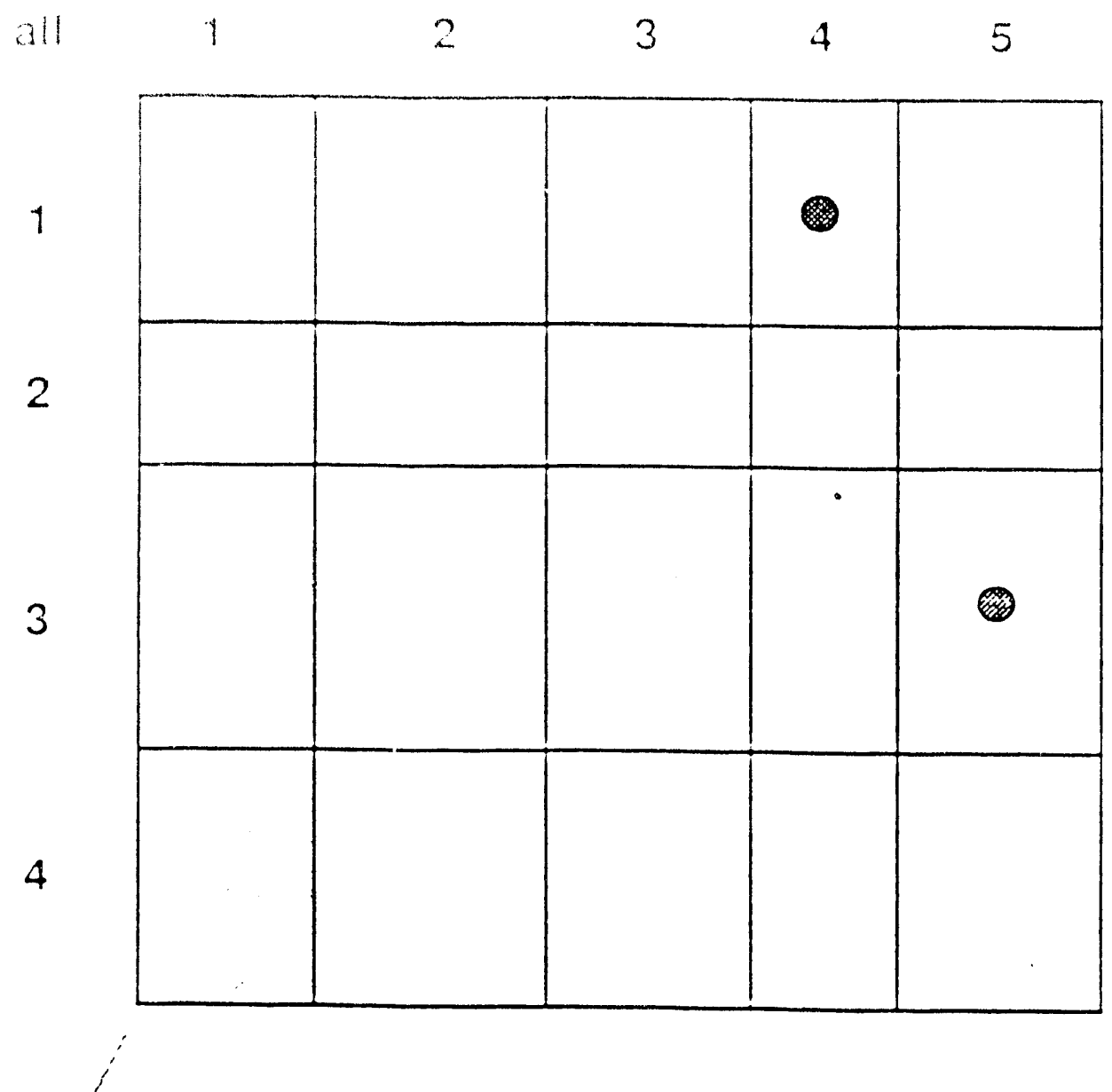

Well Options
\begin{tabular}{|l|}
\hline Add Well \\
\hline Remove Well \\
\hline Examine Well Data \\
\hline avor \\
\hline
\end{tabular}

Type Of Well

\begin{tabular}{|l|}
\hline Raie Dependent Producer \\
\hline Pressure Dedendent Producer \\
\hline Rate Dependent Injecior \\
\hline Pressure Dependent Injector \\
\hline acort \\
\hline
\end{tabular}

Fiqure 17

Entenng Recurrent Data 
done Well Characteristics

\begin{tabular}{|l|c|}
\hline Injection Concentrations & \\
\hline Injection Rates & \\
\hline Layers & \\
\hline Skil, Facior & $\frac{2.5}{8.75 \text { inches }}$ \\
\hline Well Radius & \\
\hline
\end{tabular}

cone Injection Rates

\begin{tabular}{|l|l|}
\hline Water & 30 bbls/day \\
\hline Oil & \\
\hline Suriactant & \\
\hline Polymer & \\
\hline Alcohol 1 & \\
\hline
\end{tabular}

Layers

\begin{tabular}{|c|}
\hline 1 \\
\hline 2 \\
\hline abort \\
\hline
\end{tabular}

Fiaure 18

Well Characeristics 


\section{Production History Plots}

\begin{tabular}{|l|}
\hline Oli Production \\
\hline Water Production \\
\hline Suriactant Procuctiont \\
\hline abort \\
\hline
\end{tabular}

\section{Water Production Icon}

\section{pick Production History}

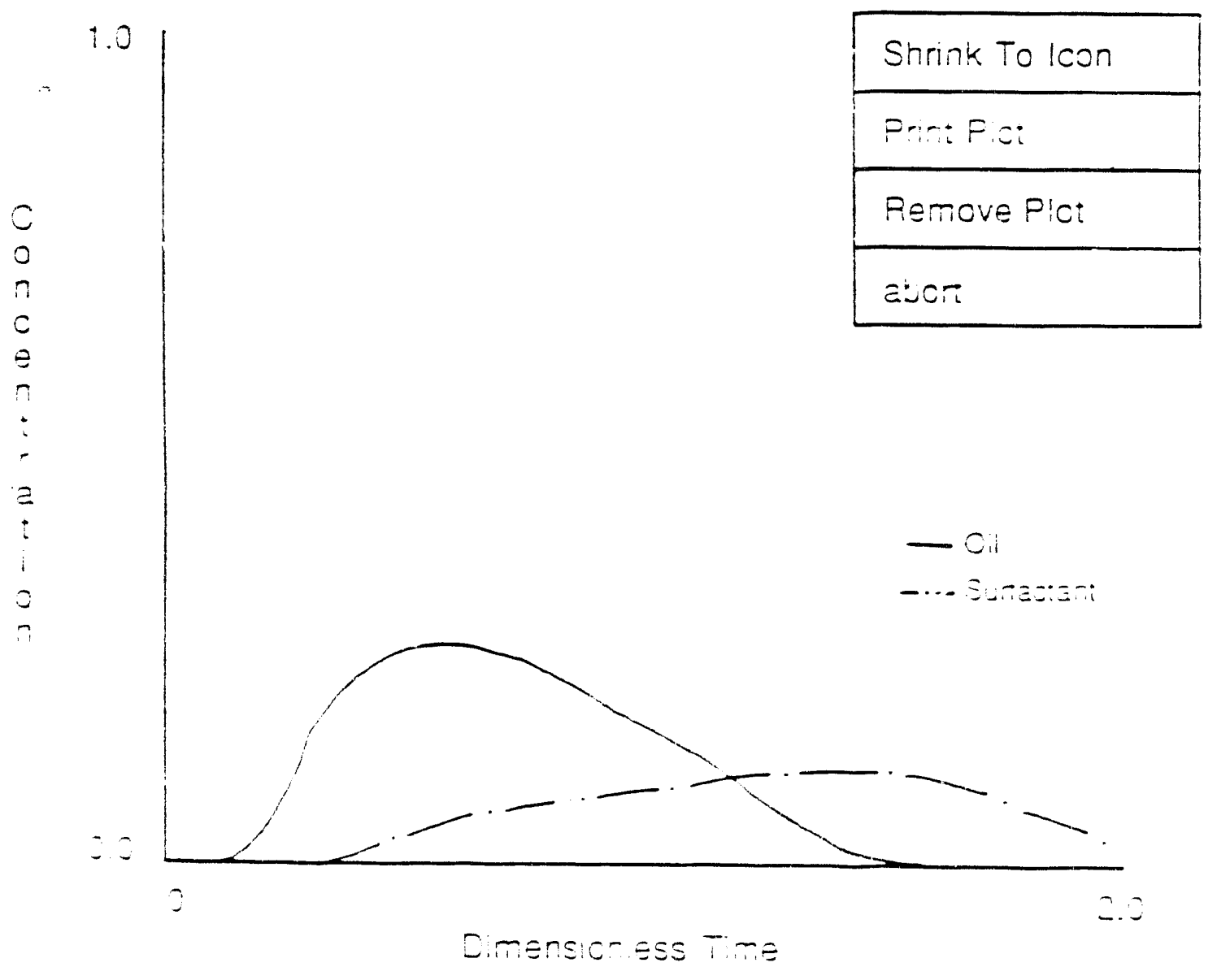

Figure 19

Froduction History Plot 


\section{Contoured Profiles}

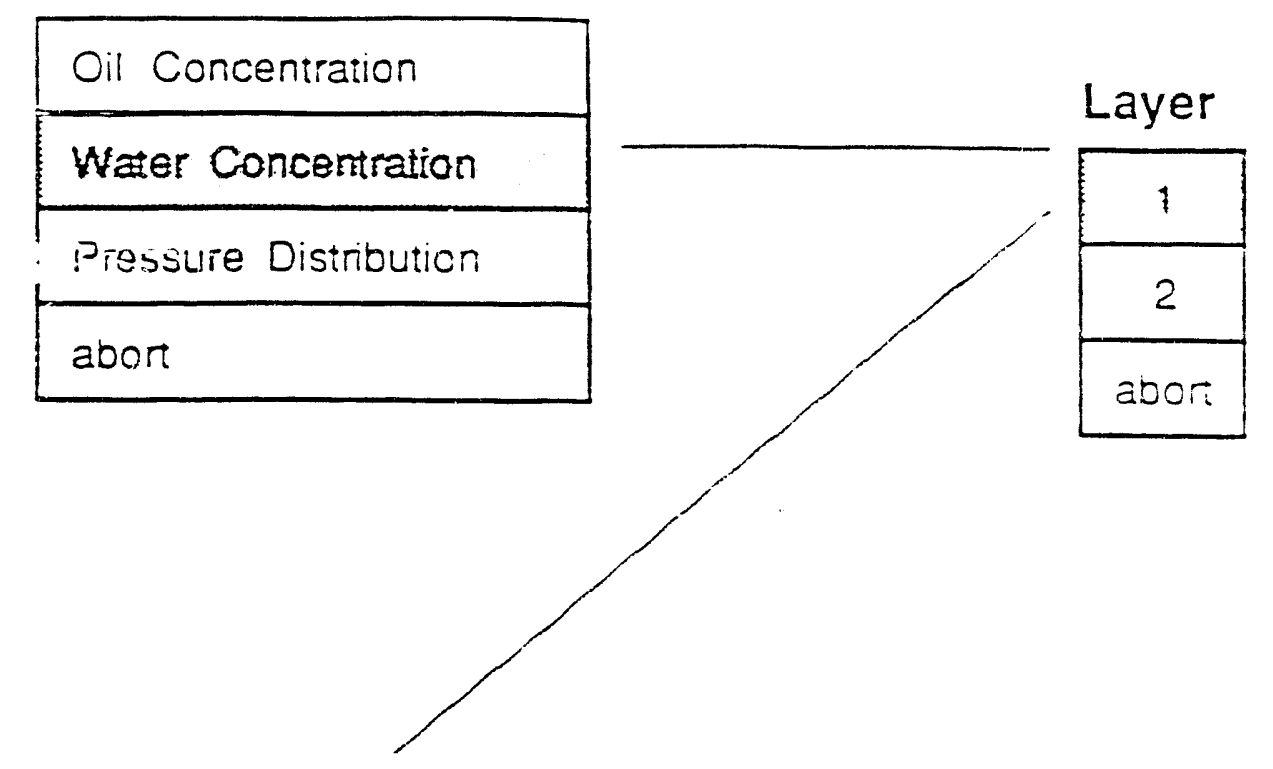

pick Water Concentration - Layer 1

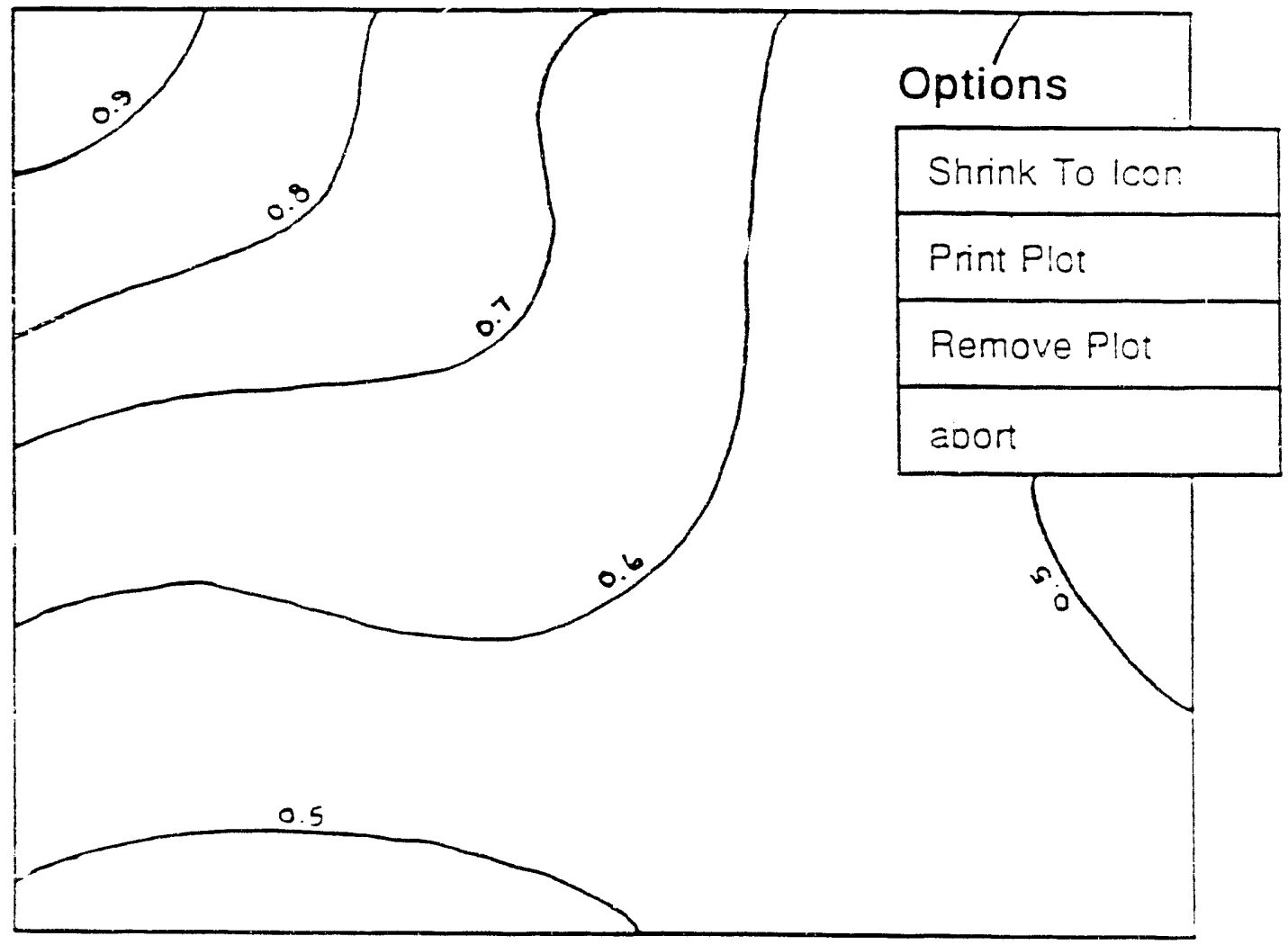

Figure 20

Contoured Protile P!ot 


\section{TASK 3 - $\mathrm{CO}_{2}$-SURFACTANT PHASE BEHAVIOR}

\section{Subtask 3a - Microemuisions and $\mathrm{CO}_{2}$-Surfactant-Water Interactions Diffusion in Microemulsions}

\section{Introduction}

The study of diffusion in microemulsions and surfactant solutions has appeared prominently in the literature because diffusion coefficients are necessary for describing mass transfer in these systems and also because diffusion data can be used to probe their microstructure. Papers dealing with diffusion in microemulsions which have appeared in the literature can be grouped into two categories, self-diffusion which occurs in the absence of bulk concentration gradients, and nonequilibrium or gradient diffusion. The former category of diffusion studies is by far the more predominant, and it includes the Fourier transform NMR investigations pioneered by Stilbs, Lindman, and their co-workers (1981 and 1984). Other methods used to study self-diffusion include the use of radioactive isotope tracers (Lam and Schechter, 1987) and dyes solubilized inside swollen micelles (Armstrong et al., 1988). By comparison, diffusion studies of microemulsions in the presence of concentration gradients are relatively scarce. A notable example of this category are papers by Miller and co-workers (Benton et al., 1986) which employ diffusion path theory to study mass transfer and the formation of intermediate phases when aqueous surfactant solutions are contacted with oil. Experiments using videomicroscopy techniques also allowed these investigators to view intermediate phase growth, interface motion, and spontaneous emulsification. Nonequilibrium diffusion results are directly applicable to diffusional processes that result from concentration gradients such as the industrial applications mentioned earlier.

The motivation for the study presented here arises from a thecry of diffusion for microemulsions proposed by Lam and Schechter (1987). This theory was compared with an experimental study of tracer diffusion in microemulsions and was found to show good agreement with experimental results. The aim of this present work is to extend the predictions of the Lam model in order to examine the more general case of nonequilibrium diffusion when microemulsions of unlike composition are contacted. In this paper, an experimental study of nonequilibrium diffusion in a nonionic micioemulsion system is compared with theoretical predictions bused on the theory of diffusion. 


\section{Theory}

The theory of diffusion in microemulsions presumes that the microstructure can be described as a dispersion of spherical droplets in a continuous phase where the the continuous and droplet phases are separated by a layer of surfactant adsorbed at the droplet interface. Material may exchange between droplets and the continuous phase on a time scale much faster than diffusion. Local equilibrium is thus assumed, and a component $i$ in the microemulsion can partition between the continuous and droplet phases. The total flux of component $\mathrm{i}$ in the microemulsion is (Lam and Schechter, 1987)

$$
-\mathrm{J}_{\mathrm{i}}^{\mathrm{T}}=\mathrm{D}_{\mathrm{i}}^{\mathrm{f}} \nabla \mathrm{C}_{\mathrm{i}}^{\mathrm{f}}+\left(\frac{\mathrm{eZ}_{\mathrm{i}} \mathrm{D}_{\mathrm{i}}^{\mathrm{f}} \mathrm{C}_{\mathrm{i}}^{\mathrm{f}}}{\mathrm{kT}}\right) \nabla \psi+\mathrm{D}_{\mathrm{m}}\left\{\nabla \cdot\left(\phi_{\mathrm{d}} \mathrm{C}_{\mathrm{i}}^{\mathrm{d}}\right)+\frac{\mathrm{e} \mathrm{Z}_{\mathrm{m}}}{\mathrm{kT}}\left(\phi_{\mathrm{d}} \mathrm{C}_{\mathrm{i}}^{\mathrm{d}}\right) \nabla \psi\right\}
$$

where $D_{i}^{f}$ is the molecular diffusion coefficient of component $i$ in the continuous phase, $D_{m}$ is the diffusion coefficient of the droplets, $C_{i}^{f}$ is the concentration of $i$ in the continuous phase, $C_{i}^{d}$ is the concentration of $i$ inside the droplets, $Z_{i}$ is the valence of component $i, Z_{m}$ is the valence associated with a droplet, $\phi_{\mathrm{d}}$ is the volume fraction of the droplets, and $\psi$ is the electrical potential. This expression includes the contribution of mass transfer in both the continuous and droplet phases. It can be used to calculate diffusion coefficients for both tracer self-diffusion and nonequilibrium gradient diffusion when fundamental parameters such as molecular diffusion coefficients and thermodynamic partition factors are input. The method of calculating tracer diffusion has been outlined in a previous paper (Lam and Schechter, 1987) and, because bulk gradients do not exist under those conditions, a closed-form expression for the effective diffusion coefficient of component $i$ is possible.

However, it is not possible to obtain a closed form solution to the nonequilibrium problem. Because of the microstructure inherent in surfactant systems, inserting the mass flux expression Eq. (1) into the continuity equation for component i results in a system of two coupled nonlinear PDE which must be solved numerically subject to appropriate boundary conditions. Because of the simplicity of modeling in one space dimension, the open-ended capillary technique was chosen as the experimental technique for this study. In the past. this method was used extensively to study tracer self-diffusion in liquids, but for that application it has been superseded in recent years by techriques such as Fourier transform NMR. However, in spite of the laborious experimental procedures involved in 
this technique, the open capillary experiment proves to be most suitable for studying nonequilibrium diffusion in microemulsions.

A component material balance in the absence of convection is

$$
\frac{\partial \mathrm{C}_{\mathrm{i}}}{\partial \mathrm{t}}=-\nabla \cdot \mathrm{J}_{\mathrm{i}}^{\mathrm{T}}
$$

where $C_{i}$ is the total concentration of $i$ per unit volume of microemulsion. The initial and boundary condirions associated with Eq. (2) are

$$
\begin{array}{ll}
C_{i}(x, 0) & =C_{i}^{c a p}(0) \\
\nabla C_{i}(0, t) & =0 \\
C_{i}(L, t) & =C_{i}^{\text {bulk }}
\end{array}
$$

where $C_{i}^{c a p}(0)$ is the concentration of material initially present in the capillary tube while $C_{i}^{\text {bulk }}$ is the concentration of component $i$ in the bulk solution. The length of the capillary tube is designated by $L$. The volume flux of the dispersed or droplet phase in the microemulsion is

$$
-J_{d}=D_{m} \nabla o_{d}+\frac{e Z_{m} D_{m} \phi_{d}}{k T} \nabla \psi
$$

This equation contains the assumption of constant droplet radius with respect to position and time. In order to calculate diffusion in microemulsions, Eq. (2) must be solved simultaneously with the continuity equation for the dispersed phase, which is

$$
\frac{\partial \phi_{d}}{\partial t}=-\nabla \cdot J_{d}
$$

Initial and boundary conditions for this equation are 


$$
\begin{aligned}
& \phi_{d}(x, 0)=\phi_{d}^{(a) l}(0) \\
& \nabla_{d}(0, t)=0 \\
& \phi_{d}(L, t)=\phi_{d}^{\text {bulk }}
\end{aligned}
$$

where $\phi_{\mathrm{d}}^{\mathrm{cap}}(0)$ is the initial dispersed phase volume fraction of the capillary solution and $\phi_{\mathrm{d}}^{\text {bulk }}$ is the dispersed phase volume fraction of the bulk solution. Equations (2) and (5) are solved numerically using the method of lines. We used the IMSL subroutine DPDES which solves parabolic partial differential equations. This algorithm discretizes the space dimension using cubic Hermite polynomials, thus, generating a series of first-order ordinary differential equations which are integrated in time. For all simulations, 100 evenly spaced grid points were used in the solution domain. Numerical solution generates the discrete profiles $C_{i}(x, t)$ and $\phi_{d}(x, t)$. These profiles are then integrated across the capillary tube length to yield their average values. By using input conditions which have an analytical solution, it was found that the accuracy of average concentrations which are determined numerically from this algorithm is within $0.2 \%$ of the accepted values. This numerical simulation permits a direct comparison of solution compositions that are obtained from the diffusion theory with those obtained from experimental data.

\section{Experimental}

A schematic of the open capillary experimental setup is shown in Fig. 1. This technique involves measuring the change in concentration of microemulsion in a small capillary tube in which the open-end is inserted into a bulk solution which contains microemulsion of a different composition. The experiment is begun when the capillary tube is inserted into the bulk. During the experiment, material diffuses into and out of the capillary tube while the two solutions are in contact. The run is ended after a known time when the tube is removed from the bulk solution. The contents of the tube are then carefully removed and analyzed to determine their composition. The initial compositions of capillary and bulk solution are also analyzed for each experiment.

The entire experimental assembly in Fig. 1 is located in an air bath where the temperature is fixed at $25^{\circ} \mathrm{C}$. The diffusion cell which contains the bulk solution is a glass jar with a plastic screw-on lid. The capillary tubes which are inserted into the bulk solution are secured vertically from the top at the lid. The glass capillary tubes used for these 
experiments, manufactured by Basic Glass Products, Lone Grove, Oklahoma, were 1/16" $(1.59 \mathrm{~mm}) \mathrm{OD}, 0.51 \mathrm{~mm} \mathrm{ID}$, and $3.1 \mathrm{~cm}$ in length. After cleaning, one end of each tube was flame-sealed to prevent evaporation of capillary solution during the experiment. Each tube was filled completely with solution using a microsyringe with a needle that could be inserted inside the tube all the way to the closed-end, and care was taken so that no bubbles remained in the filled tubes. After filling with capillary solution, the closed end was secured to the lid.

Because immersion and removal of the capillary tubes from bulk solution by hand could generate convection and skew the resulting concentration profiles, a procedure was developed to control the liquid level in the diffusion cell. A $50 \mathrm{ml}$ syringe was connected by plastic tubing to the cell containing the bulk solution, and by manually drawing back or by depressing the syringe plunger, one can lower or raise the liquid level of bulk solution in the cell to minimize convection. Further details concerning the experimental apparatus and procedure can be found elsewhere (McGreevy, 1989).

Compositions of samples from this experiment were determined by gas chromatography, using a Hewlett-Packard 5890A gas chromatograph with auxiliary 3393A integrator and 7673A automatic sampler. Each experiment involved the analysis of three samples: the bulk solution, the solution initially placed in the capillary tubes, and the resulting capillary solution obtained at the conclusion of the experiment. The compositions of the bulk and initial capillary solutions are known prior to the experiment while the unknown final capillary solution composition was determined in the following manner. The weight fraction of each component in the unknown solution was calculated using the ratio of its peak area to the peak area of the known initial capillary solution. This procedure of analyzing the composition of an unknown sample relative to that of a known standard is valid provided that the response factor, the ratio of peak area to weight fraction, is constant. We determined that this was the case for all components in the concentration ranges used in our diffusion experiments. The volume of sample taken from the capillary tubes after each diffusion experiment is typically $5 \mu \mathrm{l}$. Because the vials used for the gas chromatograph autosampler require at least $300 \mu$ l of solution, the samples obtained from experiments were diluted with $350 \mu \mathrm{l}$ of methanol or acetonitrile solvent.

The open capillary experiment was calibrated using 2.174 methanol in water solution for which the binary diffusion coefficients are known (McGreevy, 1989). In our experiment, 2.174 methanol was placed in the capillary tubes and contacted with pure water as the bulk solution. The concentration profiles measured in our experiment were within $2 \%$ of those calculated from the diffusivity value in the literature, and the reproducibility of 
our runs was $\pm 3 \%$. This accuracy and reproducibility is satisfactory for our purposes in this study.

\section{Materials}

The choice of microemulsion system for this diffusion study was made in such a way that the diffusion theory cou $\mathrm{d}$ be tested under differing conditions of bulk composition gradients. In this work, a nonionic surfactant microemulsion system is modeled so that predictions of the diffusion theory can be examined under conditions where bulk concentration and microstructure gradients exist but where the gradient of electrical potential is everywhere zero. Subsequent results will present the modeling and experiments perforned on an ionic surfactant system where both concentration and electrostatic gradients exist.

The nonionic microemulsion system studied is $\mathrm{CO}-850$-water-benzene-phenol where $\mathrm{CO}-850$ is a blend of polyethoxylated nonylphenols manufactured by GAF with an average ethylene oxide chain length of 20. Benzene obtained from Fisher Scientific and phenol from MCB Reagents were each of $99 \%$ purity and used as received. A clear single phase region is located at the water-rich end of the phase diagram for the CO-850-waterbenzene-phenol system. The fourth component phenol is not essential in creating a stable single-phase microemulsion but rather has been chosen as an additive to this system to demonstrate the existence of countergradient diffusion. More will be said about this in the next section. The solubility of phenol in the CO-850-water-benzene microemulsion was determined by selecting a number of three component solutions within the single-phase region and titrating them with phenol. The solutions were agitated after each addition of phenol, and the solubility was determined by visual inspection in a $25^{\circ} \mathrm{C}$ temperature bath. All of the microemulsions tested had phenol solubility of greater than $1.0 \mathrm{wt} \%$ which is a high enough concentration for the purposes of this study.

The experiments reported here contact microemulsions dilute in the volume fraction of the dispersed phase (those solutions closest to the water vertex of the phase diagram) with solutions having a high composition of dispersed phase (those farthest from the water vertex but still in the clear region). Tracer self-diffusion measurements on the dilute microemulsion systems strongly suggest that the microstructure in this region is in the form of droplets in a water-continuous phase. Therefore, a dilute microemulsion was chosen to be the capillary solution while concentrated microemulsions of varying phenol content were assigned as the bulk solution in experiments. 
The experimental conditions were chosen such that the phenol content of the capillary solution was held constant at 0.5 wt $\%$ while its concentration in the bulk was assigned values ranging from 0.1 to $1.0 \mathrm{wt} \%$. In order to numerically simulate the diffusion experiment under these conditions, we required the following kinds of input parameters: molecular self-diffusion data for water and phenol in water solvent; the micelle or droplet diffusion coefficient for the capillary solution; partition coefficients for water, phenol, and benzene in the capillary solution to describe their dynamic equilibrium between the continuous and droplet phases: the concentrations of water, phenol, and benzene in the capillary and bulk solutions: and the dispersed phase volume fractions in the initial capillary and bulk solutions. The self-diffusion coefficient of water is $2.22 \times 10^{-5} \mathrm{~cm}^{2} / \mathrm{sec}$ (Mills, 1971) while the tracer diffusion coefficient of phenol in water with CO-850 present below the CMC, measured by the Taylor dispersion technique (Taylor, 1953) is $8.2 \times 10^{-6}$ $\mathrm{cm}^{2} / \mathrm{sec}$. The micellar diffusion coefficient of the capillary solution, also determined by this method, is $1.25 \times 10^{-6} \mathrm{~cm}^{2} / \mathrm{sec}$. Partitioning of each component between the continuous and droplet phases can be expressed by the dimensional partition factor $\beta_{i}$

$$
\beta_{i}=\frac{C_{i}^{d}}{C_{i}^{f}}
$$

Water is found entirely in the continuous phase so that $\beta_{w}$ is equal to zero. Since hydrophobic benzene is solubilized almost completely inside the micelle cores, $\beta_{b}$ was assigned a value of $10^{6}$ for the simulations. Though this value seems arbitrary, in fact our simulations show that if $\beta_{b}$ is given values as large as $10^{30}$, it has no effect on the predicted diffusion of benzene. Phenol, on the other hand, partitions between the aqueous and droplet phases (Kandori et al., J. Colloid Interface Sci., 1989; Kandori et al., J. Phys. Chem., 1989). $\beta_{p}$ was determined from tracer diffusion measurements by a procedure given in Kandori et al. (J. Colloid Interface Sci., 1989) and its value is equal to 58.76. The concentrations of water and benzene in the capillary solution are 94.53 and $0.5 \mathrm{wt} \%$, respectively, while the initial dispersed phase volume fraction is 0.046 . The bulk solution concentrations of water and benzene are approximately 75.0 and $6.25 \mathrm{wt} \%$, respectively, while the bulk dispersed phase volume fraction is approximately 0.241 . (The exact values depend on phenol concentration in the bulk solution which is the main variable for the experiment.) With all this information in hand. modeling of the open capillary experiment 
could proceed. It should be emphasized that there are no adjustable parameters used in th calculations.

\section{Results}

Phenol Diffusion Results

Six separate experimental runs were performed. The initial capillary and bulk solution compositions of phenol, along with the resulting phenol capillary concentrations determined from theory and experiment at 16.25 hours, are shown in Table 1 . Both sets of results show a similar decreasing trend in final capillary concentration of phenol as the phenol content of the bulk solution is lowered. In Run \#1, both theory and experiment show that phenol concentration rises in the capillary when it is contacted with $1.0 \%$ phenol microemulsion in the bulk. Runs \#5 and \#6 show that phenol concentration decays inside the capillary tube when contacted with microemulsions of lower phenol content in the bulk. For each of these runs, phenol diffusion is occurring in the direction of the concentration gradient, as would be expected for simple liquids. However, the experimental data for Runs \#2, \#3, and \#4 reveal that during the period of measurement phenol concentration decreases in the capillary tube despite the fact that the bulk solutions are more concentrated in phenol. Phenol diffusion in these runs is occurring against the concentration gradient. The results ohtained from the theory also predict this countergradient diffusion of phenol for Runs \#3 and \#4.

These same results for phenol are plotted in Fig. 2 as normalized phenol concentration in the capillary tube as a function of bulk phenol concentration. The countergradient results are found for values of normalized concentration greater than unity. The discontinuify in normalized concentration of phenol shown in this plot occurs when the bulk concentration of phenol closely approaches that initially present in the capillary, $0.0532 \mathrm{M}$, which makes normalized concentration tend toward positive or negative infinity. These results show a very good agreement between theory and experiment which is significant, considering the surprising nature of the phenol diffusion behavior.

Figure 3 shows simulated phenol concentration profiles within the capillary tube at various times based on the conditions of Run \#3 which corresponds to the curve for 16.25 hours. The node at the extreme right is positioned at the open end of the tube, and its value is equal to the bulk solution concentration of phenol, $0.063 \mathrm{M}$ or $0.6 \%$, at all times. The remaining points inside the tube are initially at the same concentration of $0.0532 \mathrm{M}$ and then change as a function of time. What occurs during the course of the experiment, according to this simulation, is quite remarkable. A well or minimum of phenol concentration 
develops within the tube near the open-end. As the experiment proceeds, this well moves toward the middle of the tube and begins to broaden as the curves for 16.25 and 69.4 hours illustrate. With further passage of time, the minimum in the center of the tube begins to disappear and the curves for 111.1, 166.7, and 278.8 hours indicate that phenol concentration falls near the closed end of the tube. During these intermediate times, phenol is being depleted near the closed end while its concentration is building up near the openend. Ultimately, at very long times, the phenol concentration in the capillary tube will become equal to its concentration in the bulk solution. The existence of a concentration minimum at the earlier times implies that two opposing mechanisms of phenol diffusion are occurring in this system, and we shall discuss these in more detail in a later section.

\section{Water and Benzene Diffusion Results}

The normalized capillary concentrations determined for water and benzene are shown in Figs. 4 and 5 as a function of bulk phenol composition. All normalized concentrations in these plots are found to be less than unity. The results for these components (especially benzene) seem to show an even closer agreement between theory and experiment than that of the phenol case. This agreement is significant in light of the fact that the microemulsions contacted in diffusion experiments contain large concentration gradients of water and benzene.

The nature of benzene and surfactant diffusion (which are associated primarily with droplets) in microemulsions is illustrated by Fig. 6 which shows droplet volume fractions as a function of position in the tube for various times. By contrast with the phenol results, the droplet composition of the microemulsion in the capillary tube increases monotonically with position and time as that solution is contacted with one in the bulk having a much higher composition of dispersed phase (benzene and surfactant).

\section{Discussion}

In considering the CO-850 microemulsion system results in Table 1 for average phenol concentrations remaining in the capillary, for every run the experimental data are slightly lower than the values predicted by the theory of diffusion. Because this difference is nearly the same in each case, we set out to determine whether there were factors in the theoretical simulations or in the experimental method that could eliminate some or all of the discrepancy. As for the theory, additional simulations showed that none of these parameters could be varied, either individually or as a group, in such a way that the 
theoretical predictions of phenol concentration could be lowered for all six runs and brought closer to experimental values. After all data were obtained, a small error in the experimental procedure was discovered; the correction of this error will raise experimental values 1 to $2 \%$ and improve the agreement with the theoretical results.

The countergradient results observed for phenol in the CO-850 microemulsion system are a most interesting phenomenon, and an explanation for them shall now be considered. In the case of the experimental data, the results reported for each run are the average of two or more separate experiments. The possibility that these counterintuitive results arise from experimental error has been considered. The correction of the error discussed in the preceding paragraph would raise all experimental values for average phenol concentrations slightly. While this would reduce the countergradient effect observed in the CO-850 system experiments in Runs \#2, \#3, and \#4 of Table 1, it would not eliminate it. The magnitude of the countergradient results for phenol in Runs \#2, \#3, and \#4 lies outside the variance for the experiment reported earlier in this paper. Furthermore, the experimental data in all six runs follow a systematic trend which agrees with the trend of results obtained from the theory. This diffusion theory also predicts countergradient results for phenol in two of the runs. In light of this, our experimental findings cannot be considered as artifacts.

The theory of diffusion was tested in numerical simulations in order to determine which input parameters have the greatest effect in producing novel results like those found in this study. It was found that the molecular diffusion coefficient $D_{i}^{f}$, the droplet diffusion coefficient $D_{m}$, and partition factor $\beta_{i}$ are what distinguish the diffusion behavior of phenol in these systems from that of water and benzene. For example, when the molecular diffusion coefficient and partition factor are each varied in simulations of the nonionic system while holding all other parameters constant, we found that values of $D_{i}^{f} / D_{m}$ greater than 6.4 and $\beta_{i}$ ranging from 5 to 70 lead to countergradient diffusion for component $i$. Both of these conditions are met by phenol for the capillary solution chosen in the CO-850 microemulsion system but not by any of the other components.

Equation (1) shows that two coupled diffusion rates contribute to the total flux. Diffusion can occur according to molecular motion in the continuous phase and also as part of the slower Brownian droplets. The partition factor couples these two mechanisms and determines which will predominate. Phenol is found in both locations and thus has two mechanisms of diffusion occurring simultaneously. How this can explain"countergradient diffusion behavior is illustrated in Fig. 7. In these plots, the capillary concentration profile for phenol located in the continuous phase, along with the profile of the volume fraction of 
droplets, are shown here for the time 16.25 hours. That portion of phenol residing in the continuous phase is diffusing out of the capillary tube while droplets are diffusing into the tube and carrying solubilized phenol with them. Because of the particular conditions which were selected for this experiment, these two mechanisms of diffusion are opposing one another, causing a minimum in total phenol concentration. Because the nature of droplet diffusion is slow relative to that in the continuous phase, the latter mechanism has a stronger influence on the mobility of phenol which is directed from the capillary toward the bulk solution. Continuous phase diffusion of phenol overcomes the effect of solubilized phenol diffusing from the bulk solution into the capillary. Thus, these competing mechanisms lead to a net diffusion behavior which is countergradient. If droplet diffusion rates are accelerated, then it may be possible to observe a maximum in the capillary tube rather than a minimum.

\section{Conclusions}

Nonequilibrium diffusion has been studied in nonionic surfactant microemulsions using the open-ended capillary experiment, and the theory of diffusion has been found to represent well the concentration profiles of water, benzene, and phenol for systems which have a droplet morphology. Gradients in concentration of each component as well as solution microstructure were present in these experiments, and the model clearly distinguishes the quantitative features of diffusion for each component. In particular, the theory is able to predict countergradient diffusion behavior for components which partition between continuous and droplet phases, and this was verified in experiments by using phenol as the partitioning additive. The open capillary experiment is shown in this study to be extremely appropriate and useful for studying nonequilibrium diffusion behavior of microstructured solutions, whereas in the past its use was largely confined to equilibrium tracer diffusion studies.

A note of caution is apprcpriate, however. A careless interpretation of the countergradient results obtained for phenol will lead to a pitfall. If one used the analytical solution to the open capillary problem, he would calcuiate for phenol a negative-valued effective diffusion coefficient, a nonphysical result. Clearly, in order to adequately describe diffusion in microemulsions under nonequilibrium conditions, it is not sufficient to consider only concentration differences of a component in a diffusion couple. Knowledge of microstructure and electrostatic gradients as well as concentration gradients is a requirement for modeling diffusion in these systems. 


\section{Alcohol Partitioning}

\section{Introduction}

Alcohols are frequently used as cosolvents in preparing microemulsions for use as enhanced oil recovery agents. These partition between the aqueous, oil, and interfacial layers. The interfacial concentration of alcohol is the crucial guantity since it is this concentration which modifies the phase behavior. A few investigations of alcohol partitioning have been reported, but these are in the temperature range of 25 to $30^{\circ} \mathrm{C}$. Since reservoir temperatures may be elevated, the influence of temperature on alcohol partitioning may be important.

\section{Experiments}

The effect of the addition of a-butanol on the micellization of dodecyltrimethylammonium bromide (DDTAD) at elevated temperatures was investigated by measurements of the tracer diffusion coefficients. The extent of alcohol partitioning into the $\mathrm{m}$ celles and its effect on micellar sizes were calculated from the diffusion coefficients.

Using the Taylor dispersion technique, the diffusion coefficients of n-butanol and $\mathrm{n}$-hexadecane were determined as functions of temperature and n-butanol molality. Hexadecane, being only sparingly soluble in water, mostly resided within the DDTAB micelles and had the same diffusion coefficient as the micelles.

The diffusion coefficients of hexadecane in 0.1 molal of aqueous DDTAB solutions are shown in Fig. 8. They increase slightly with n-butanol molality at both 50 and $75^{\circ} \mathrm{C}$, which indicates a small reduction of micellar size with the addition of n-butanol. At $100^{\circ} \mathrm{C}$, the diffusion coefficient has a maximum, implying a minimum in the micellar size. The diffusion coefficient of $n$-butanol was shown in Fig. 9 and found to decrease with increasing $n$-butanol molality.

Assuming that the CMC of DDTAB was small compared with the overall molality of DDTAB so that all DDTAB molecules exist as micelles, the $n$-butanol-to-DDTAB mole ratios in the micelles were calculated and shown in Fig. 10 as functions of $n$-butanol molality in the continuous bulk phase. The partitioning of $n$-butanol into micelles increases with the $\mathrm{n}$-butanol molality in the continuous phase and decreases with temperature.

\section{Conclusions}

i As the extent of alcohol partitioning increases and the $n$-butanol-to-DDTAB ratio is as high as 2.5 at a high n-butanol molality, the micellar sizes of DDTAB at both 50 and 
$75^{\circ} \mathrm{C}$ reduce only slightly. We conclude that some of the surfactant molecules in a micelle are actually replaced by alcohol molecules, which are shorter chain molecules compared to the DDTAB molecule and have different hydrophobic-hydrophilic behavior.

The partitioning and size variations will be compared with CMC data available in the literature so as to elucidate the effect of alcohol and temperature on micellization.

\section{References}

Anderson, J.S. and K. Saddington,: "The Use of Radioactive Isotopes in the Study of the Diffusion of Ions in Solution," J. Chem. Soc. (1949) Supplemental Issue \#2, S381.

Armstrong, D.W., R.A. Menges, and S.M. Han: "Evaluation of Dye-Micelle Binding Constants Using Diffusion Sensitive Band Broadening Effects," J. Colloid Interface Sci. (1988) 126, 239.

Benton, W.J., K.H. Raney, and C.A. Miller: "Enhanced Videomicroscopy of Thase Transitions and Diffusicnal Phenomena in Oil-Water-Nonionic Surfactant Systems," J. Colloid Interface Sci. (1986) 110, 363.

Kandori, K., R.J. McGreevy, and R.S. Schechter: "Solubilization of Phenol in Polyethoxylated Nonionic Micelles," J. Colloid Interface Sci. (1989) 132, 395.

Kandori, K., R.J. McGreevy, and R.S. Schechter: "Solubilization of Phenol and Benzene in Cationic Micelles: Binding Sites and Effect on Structure," J. Phys. Chem. (1989) $93,1506$.

Lam, A.C. and R.S. Schechter: "A Study of Diffusion and Electrical Conduction in Microemulsions," J. Colloid Inte'face Sci. (1987) 120, 42.

Lindman, B., P. Stilbs, and M.E. Moseley: "Fourier Transform NMR Self-Diffusion and Microemulsion Structure," J. Colloid Interface Sci. (1981) 83, 569.

McGreevy, R.J.: "Trüer and Nonequilibrium Diffusion in Micellar and Microemulsion Systems," Ph.D. dissertation, U. of Texas, Austin (1989)

Mills, R.: "Isotopic Self-Diffusion in Liquids," Ber. Bunsenges Phys. Chem. (1971) 75 , 195.

Pratt. K.C. and W.A. Wakeham: "The Mutual Diffusion Coefficient of Ethanol-Water Mixtures: Determination by a Rapid, New Method," Proc. 2. Soc. London (1074) Series A.336, 393

Stibs. P. and B. Lindman: "Aerosol OT Aggregation in Water and Hydroearbon Solutions from NMR Selt-Difusion Measuremenis," J. Colioid Interface. Sci. (1984) 99. 290. 
Taylor, S.G.: "Dispersion of Soluble Matter in Solvent Flowing Slowly through a Tube," Proc. R. Soc. London (1953) Series A, 219, 186.

\section{Nomenclature}

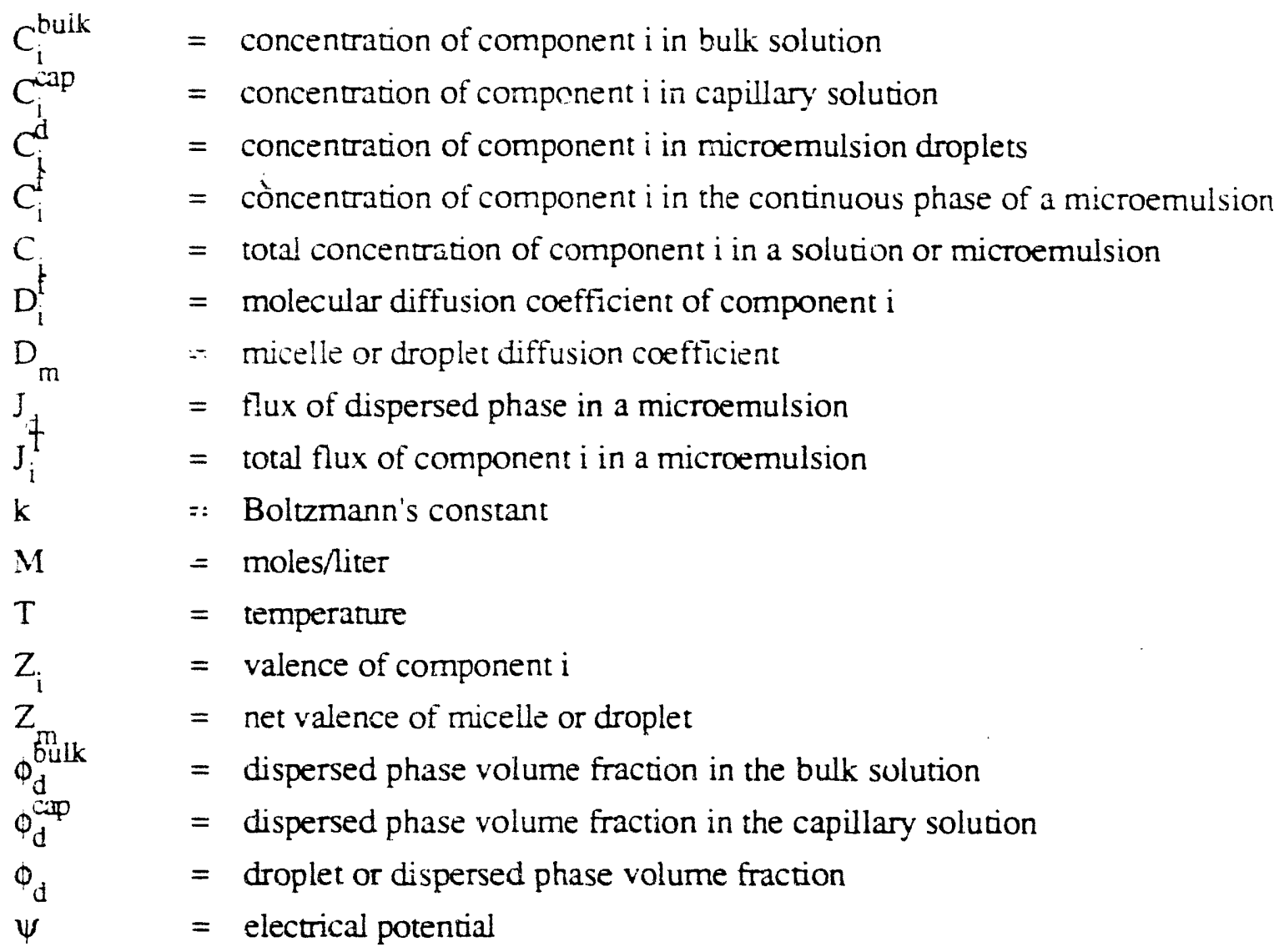


Table 1. Open Capillary Simulation and Experimental Results of Phenol Concentrations for CO-850-Water-Benzene-Phenol System.

\begin{tabular}{|c|c|c|c|c|}
\hline \multicolumn{3}{|c|}{$\begin{array}{c}\text { Initial Conditions } \\
\text { [Phenol] }\end{array}$} & \multicolumn{2}{|c|}{$\begin{array}{c}\text { Results } \\
\text { (Time }=16.25 \text { hrs.) } \\
{[\text { Phenol }]}\end{array}$} \\
\hline Run & $\underset{\left(w t^{2} c\right)}{B u l k}$ & $\begin{array}{c}\text { Capillary } \\
(\text { wt } \%)\end{array}$ & $\begin{array}{l}\text { Theory } \\
\text { (wi } \%)\end{array}$ & $\begin{array}{l}\text { Experiment } \\
\quad(w t \%)\end{array}$ \\
\hline$!$ & 1.0 & 0.5 & 0.543 & 0.520 \\
\hline 2 & 0.75 & 0.5 & 0.515 & 0.485 \\
\hline 3 & 0.6 & 0.5 & 0.498 & 0.481 \\
\hline 4 & 0.52 & 0.5 & 0.489 & 0.478 \\
\hline 5 & 0.25 & 0.5 & 0.461 & 0.437 \\
\hline 6 & 0.1 & 0.5 & 0.445 & 0.426 \\
\hline
\end{tabular}




\section{List of Figures}

Figure

1 Schematic of open capillary experimental apparatus

2 Normalized phenol concentration remaining in capillary at 16.25 hours as a function of bulk phenol concentration

3 Simulated phenol concentration profiles within capillary for various times, based on conditions of Run \#3

4 Normalized water concentration remaning in capillary at 16.25 hours as : function of bulk phenol concentration

5 Normalized benzene concentration remaining in capillary at 16.25 hours as a function of bulk phenol concentration

6 Simulated droplet volume fraction profiles within capillary for various times

7 Two competing mechanisms of phenol difrusion. Free diffusion in the continuous phase and solubilized phenol diffusing with Brownian droplete

8 The diffusion coefficients of $n$-hexadecane as functions of overall $n$-butanol molality at 50,75 , and $100^{\circ} \mathrm{C}$

9 The diffusion coefficients of $n$-butanol as functions of overall $n$-butanol molality at 50,75 , and $100^{\circ} \mathrm{C}$

10 The $n$-butanol to DDTAB mole ratios in micelles as functions of $n$-butanol molality in the continuous phase at 50,75 , and $100^{\circ} \mathrm{C}$ 


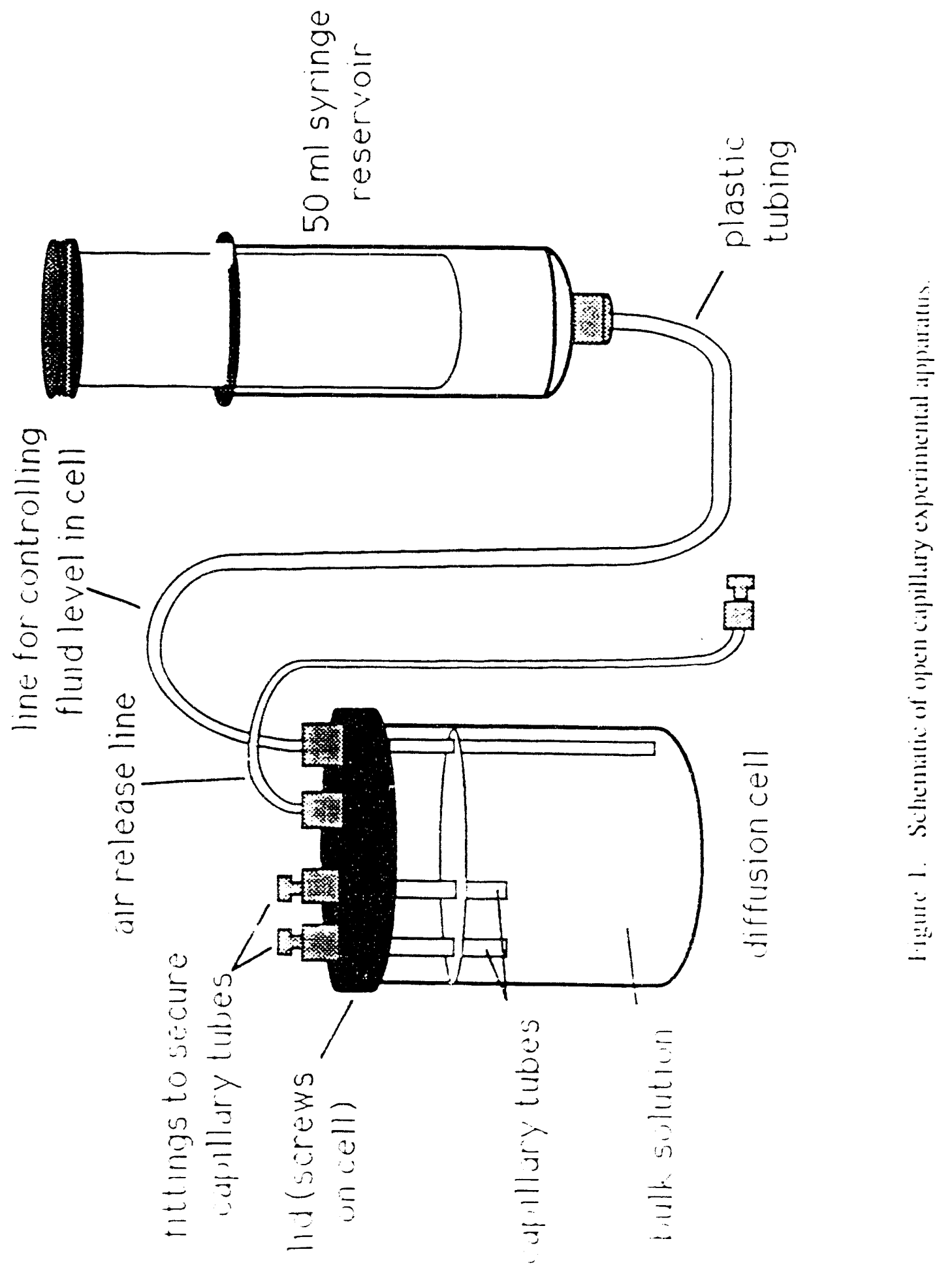




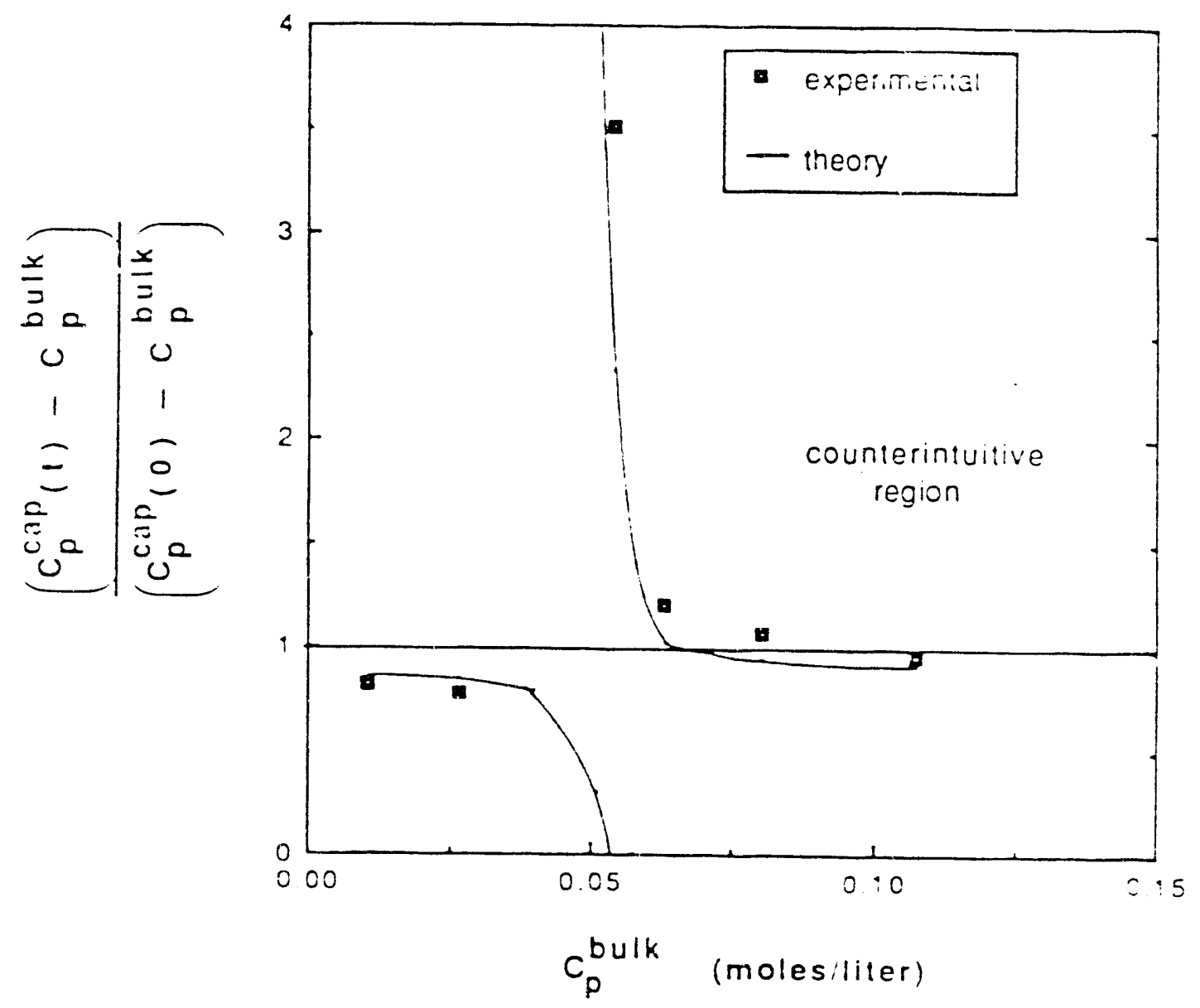

Figure 2. Normalized phenol concentration remauning in capillary at 16.25 hours as a function of bulk phenol concentration. 


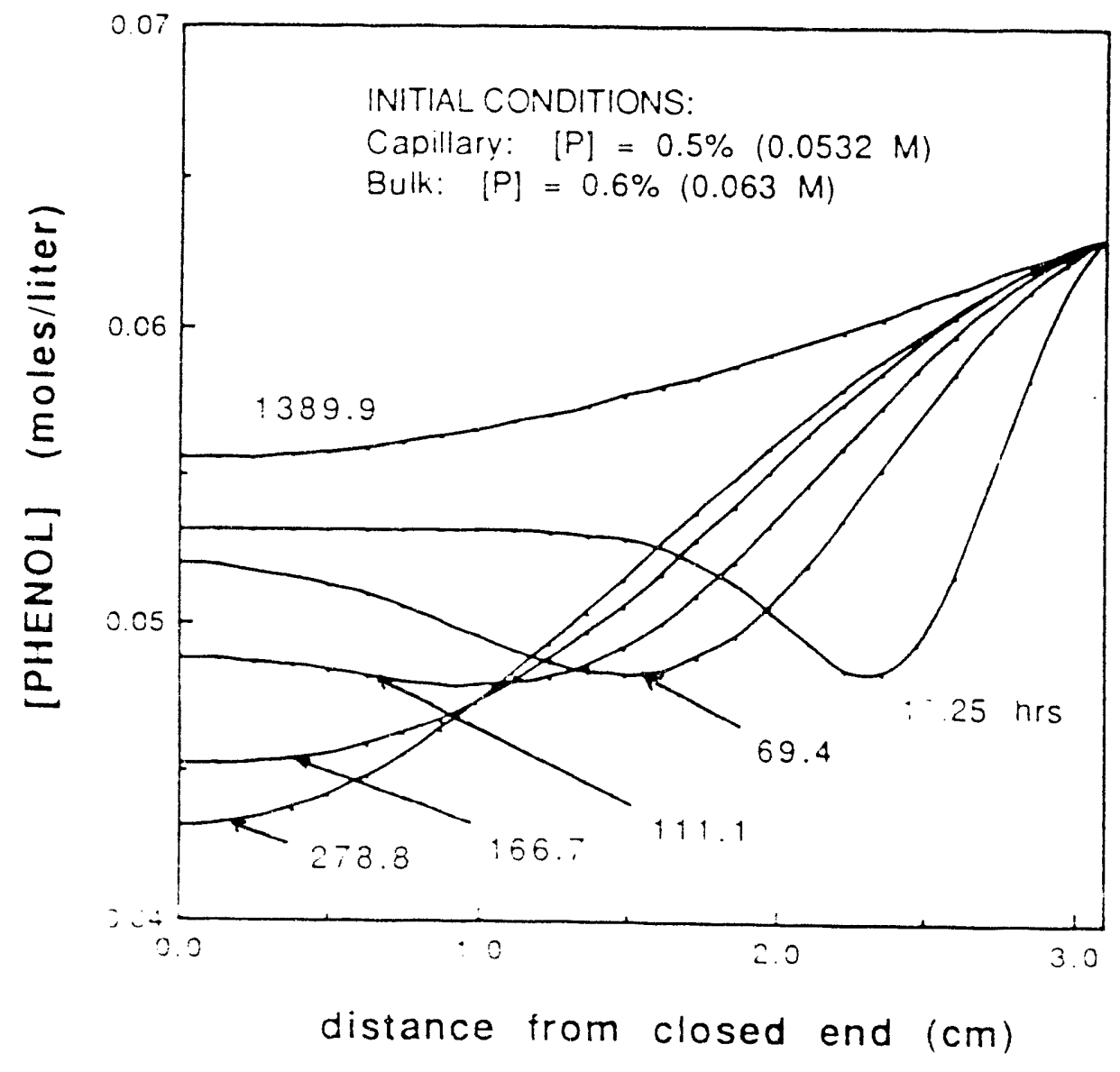

Figure 3. Simulated phenoi concentration protiles within capillary for various times, based on conditions of Run $\neq 3$. 


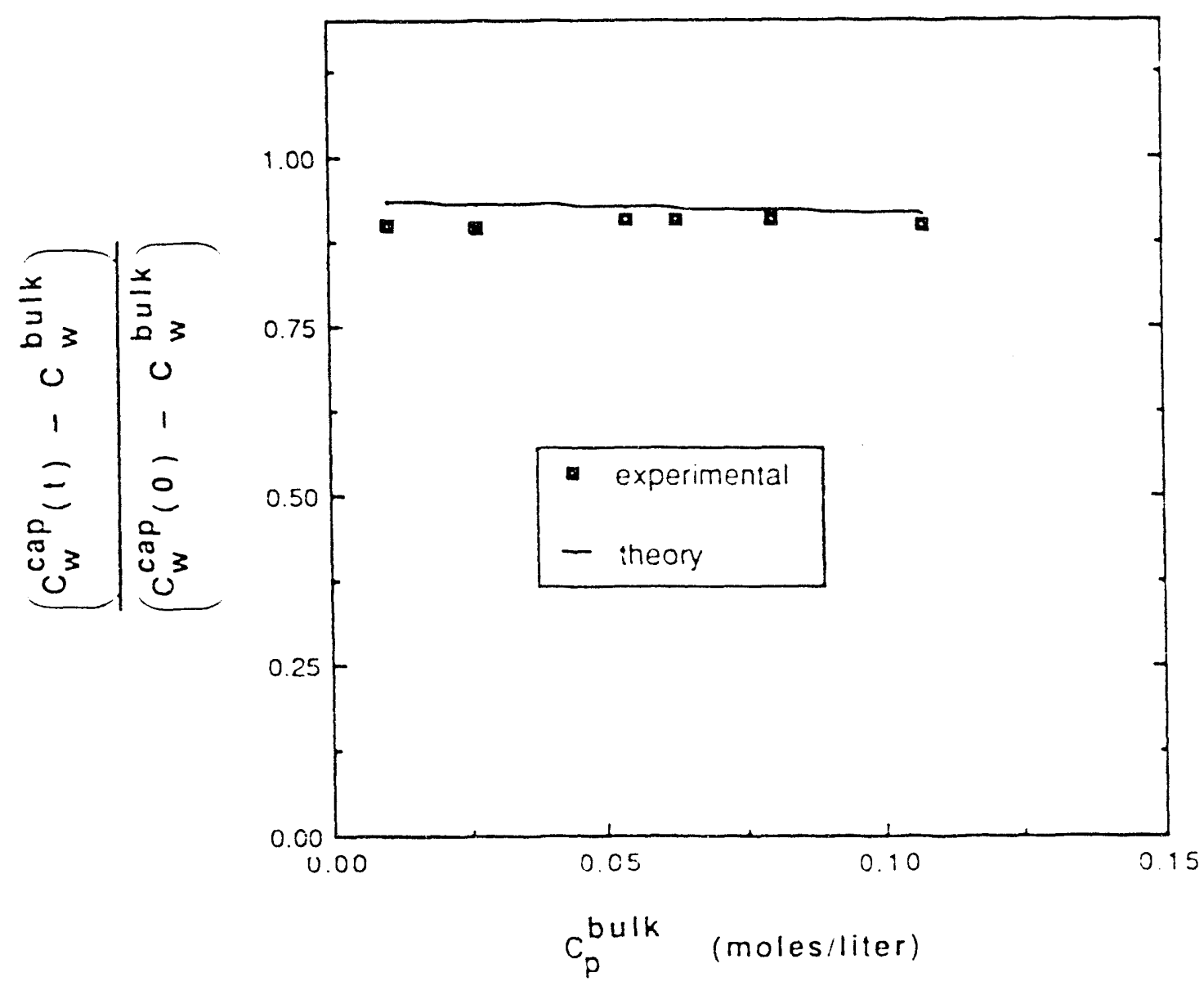

Figure 4. Normalized water concentration remaining in capillary at 16.25 hours as a function of bulk phenol concentration. 


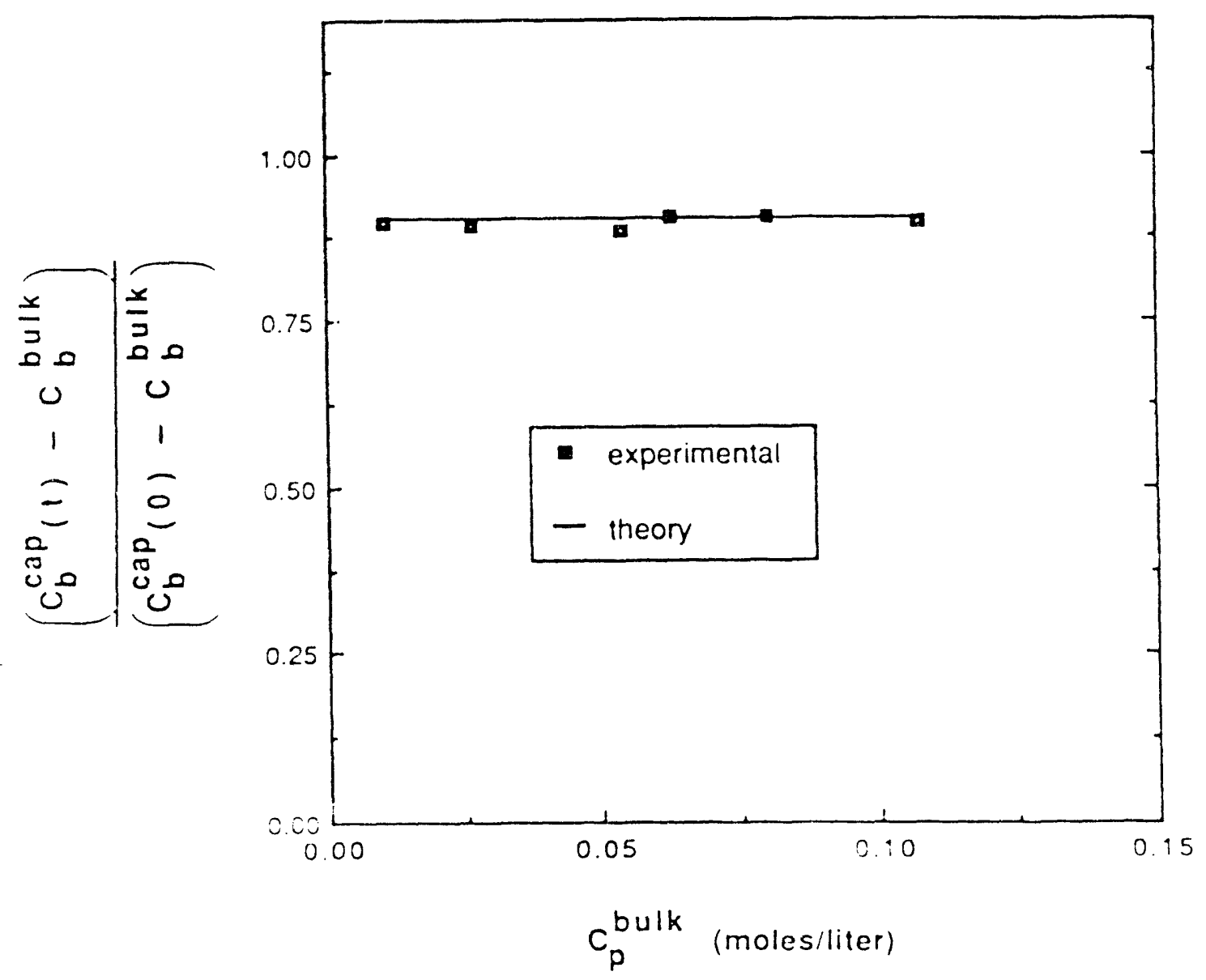

Figure 5. Vormaiized benzene concentration remaining in capillary at 16.25 hours as a function of bulk phenol concentration. 


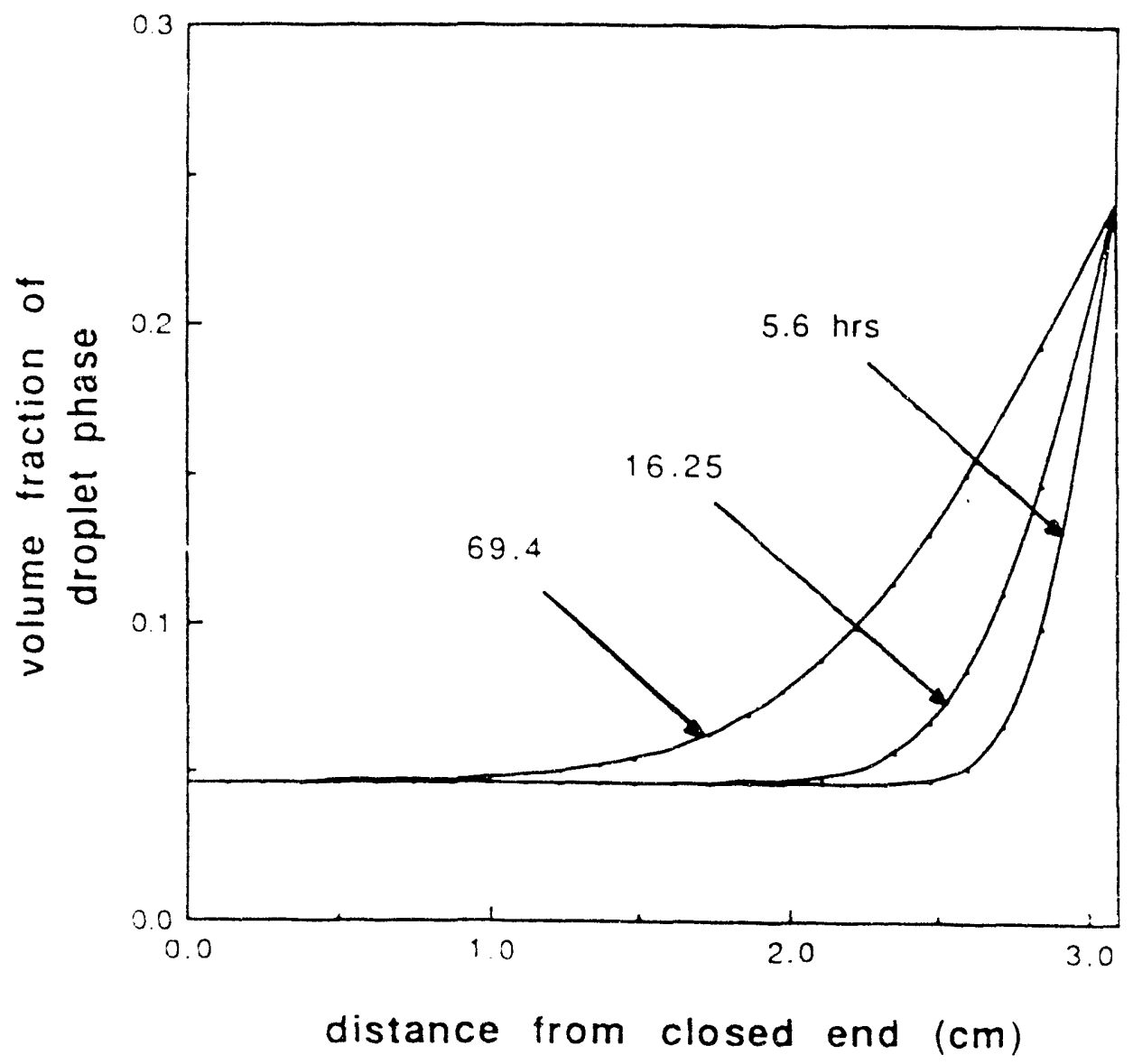

Figure 6. Simulated droplet volume fraction protiles within capillary for various times. 

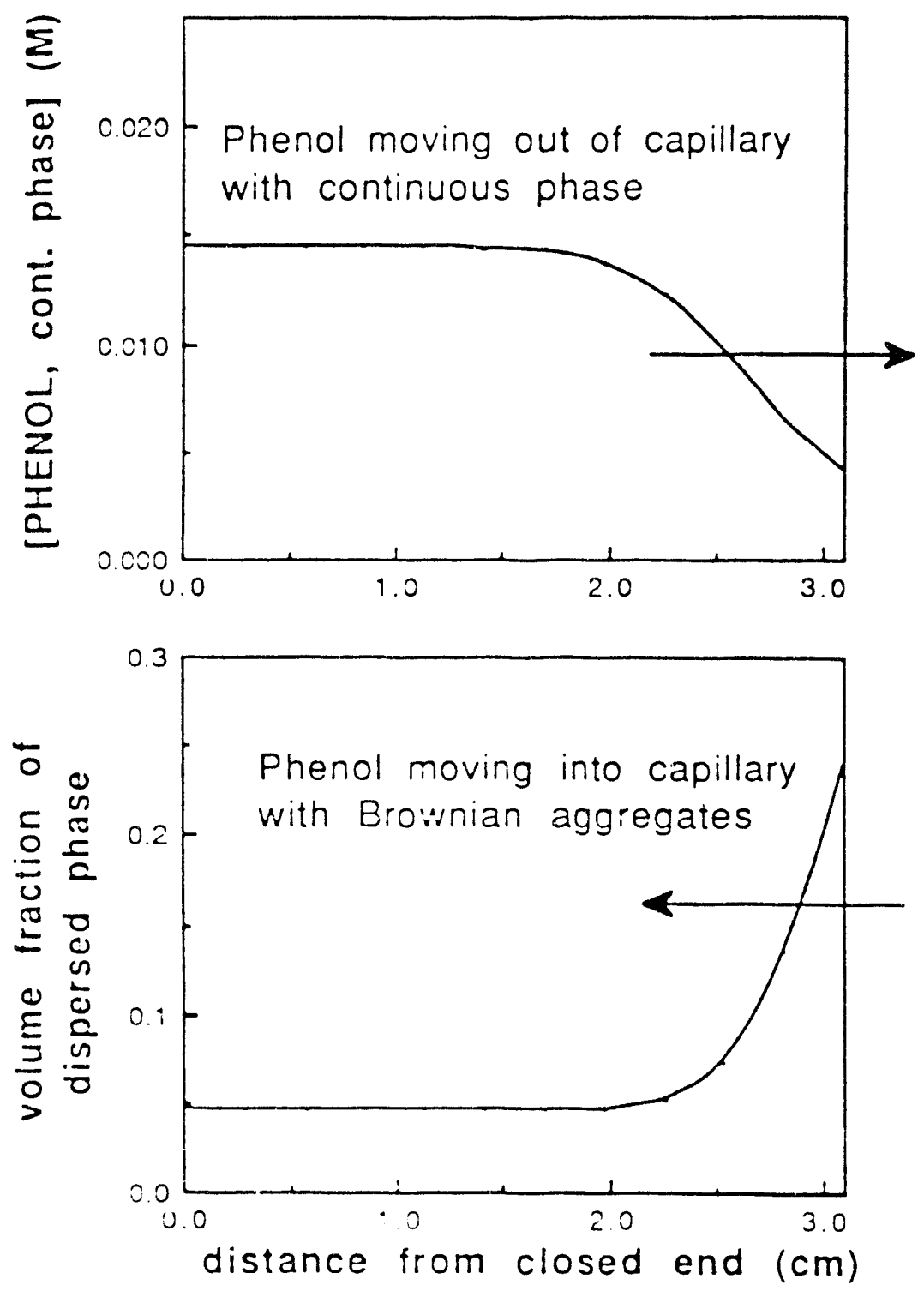

Figure 7. Two compering mechanisms of phenol diffusion. Free diffusion in the continuous phase and solubilized phenol diffusing with Brownian dropiets. 


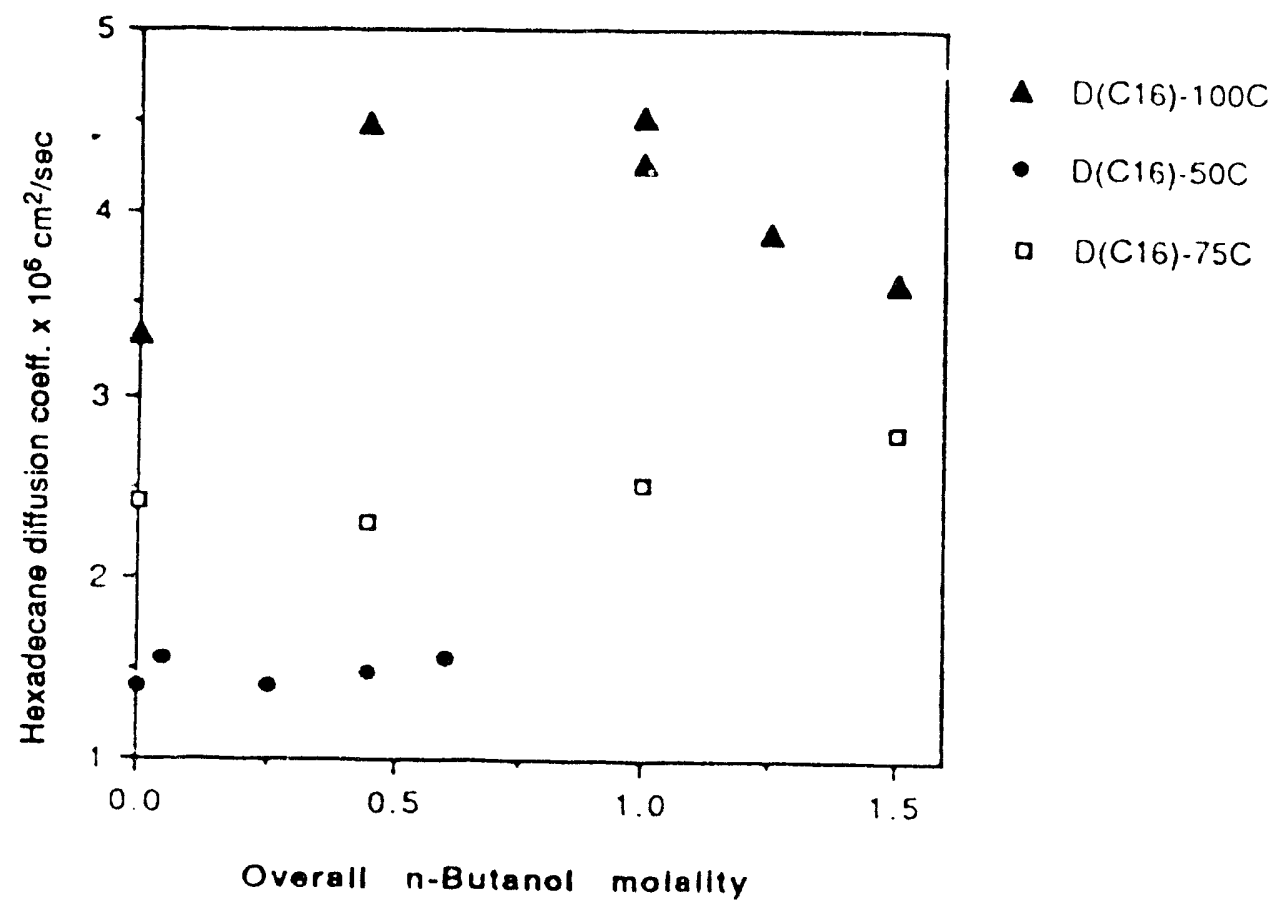

Figure 8. The diffusion coefficients of $n$-hexadecane as functions of overall n-butanol molality at 50,75 , and $100^{\circ} \mathrm{C}$. 


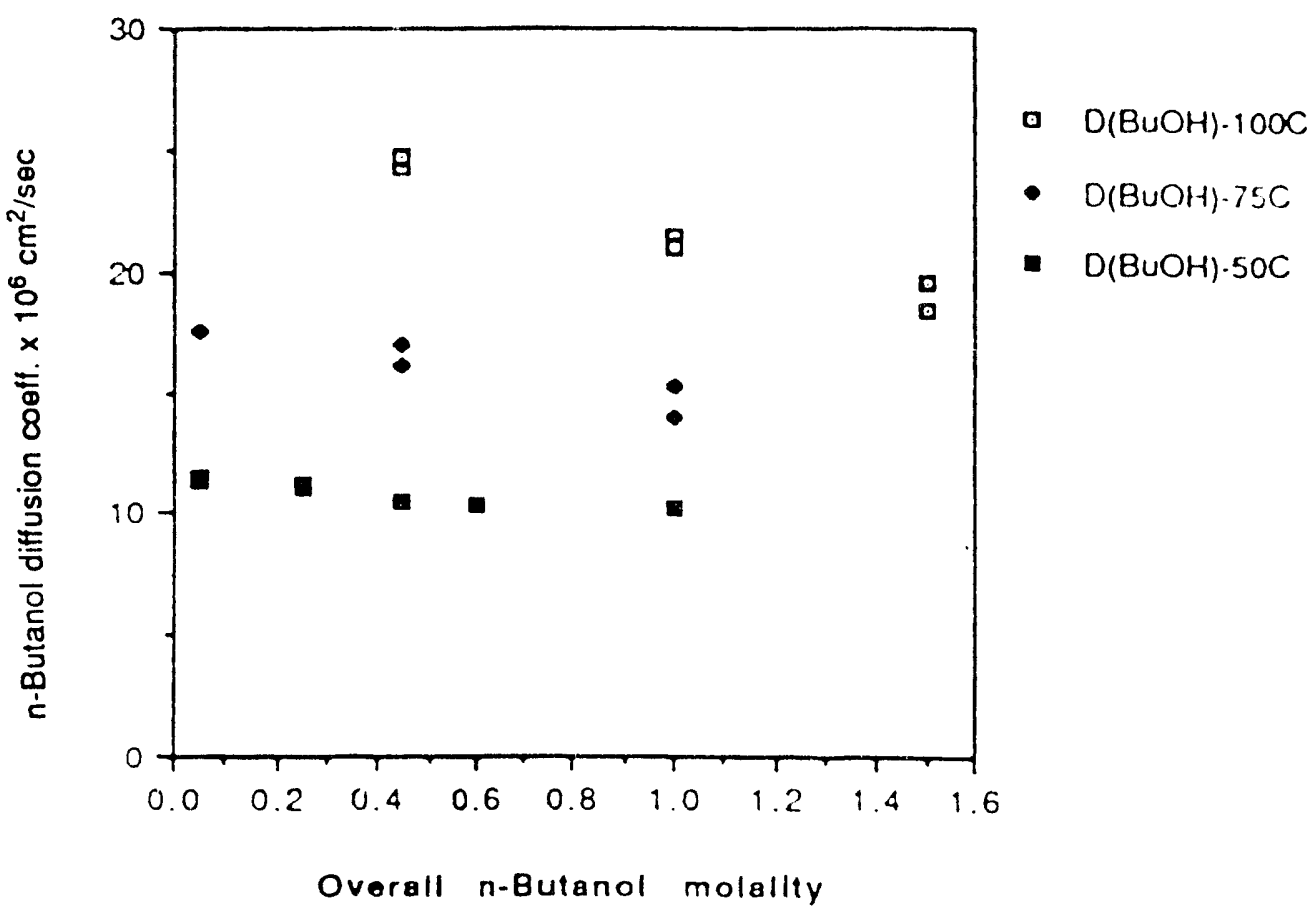

Figure 9. The diffusion coefficients of $n$-butanol as functions of overall n-butanol molality at 50,75 , and $100^{\circ} \mathrm{C}$. 


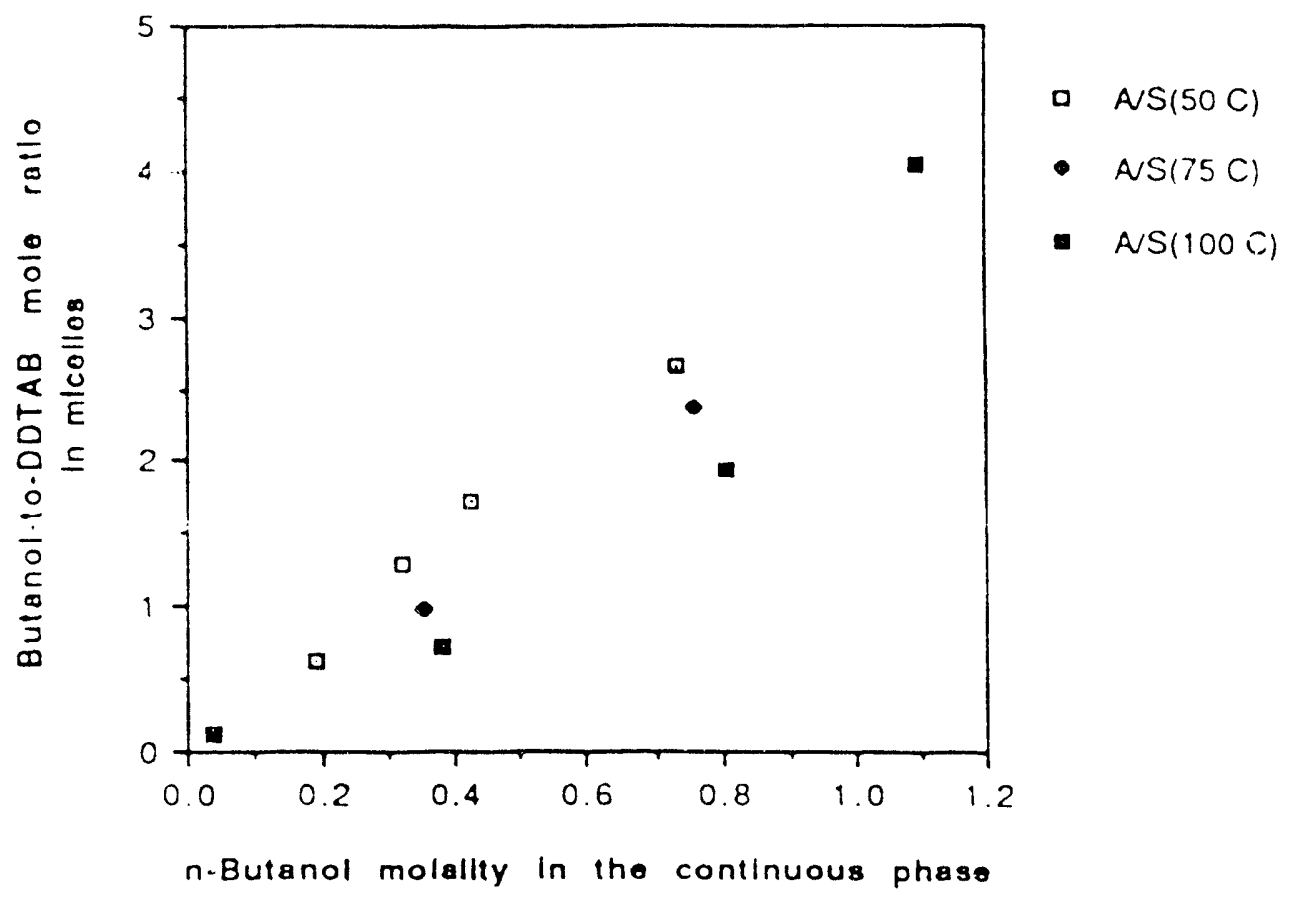

Figure 10. The n-butanol to DDTAB mole ratios in micelles as functions of n-butanol molality in the continuous phase at 50,75 , and $100^{\circ} \mathrm{C}$. 
Subtask $3 b-$ Thin Film Stability

\section{Thin Films}

\section{Introduction}

It is krown that the presence of stable films is needed to permit the formation of foam lamella within a porous medium. The stability of thin aqueous films is therefore thought to be one of the crucial features goveming mobility control by foam. It is the purpose of this study to gain an understanding of factors that cetermine the stability of thin liquid films on solid substrates. particularly of systems containing surface-active chemicals.

A microcell assembly designed to capture a single thin liquid film on a solid substrate at a known applied pressure has been constructed. The film thicknesses of n-tetradecane on a borosilicate glass surface at various disjoining pressures have been measured. and the results compared well with reported values in the literature. After some initial difficulty, stable films of aqueous electrolyte solutions have been formed and the disjoining pressures measured as a function of the film thickness. Furthermore, the possibility of the influence of the latera film size on its stability is also being investigared.

\section{Experimental}

Our schemaric of the microcell assembly is shown in Fig. 1. A fritted glass disc with pore sizes ranging from 10 to 15 microns and with a circular opening at its center is used as a "one-way valve," allowing the free passage of the werting phase but not the nonwetring phase. Porous discs with circular openings of both 1 and $2 \mathrm{~mm}$ diameter are used. The solid substrate and the tritted glass disc are enclosed in a Plexiglas cylindrical casing. A polyethylene tube connects the microcell to a manometer used to manipulate the film pressure. A Rudolph AutoEL II ellipsometer is used to measure the thickness of the thin tins.

A high optical quality borosilicaie crown giass ( $\mathrm{BK}-\mathrm{T} / \mathrm{A}$ ) made by Oriel is used as the solid substrate. Its surtace is that to within 1/20 of the wave length of sodium and has at 40-ro-20 research grade optcal finsh. Tre glass substate was cleaned with a mixure of

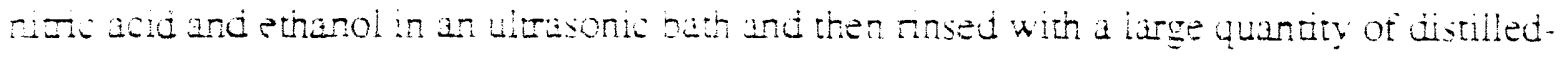
delonzed water betore use. The rest of the issembly was cleaned with a midd detergent

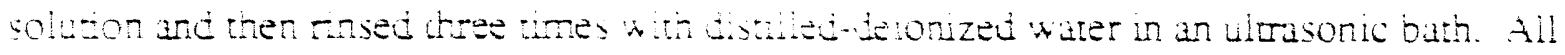


the solutions used in the experiments were filtered with a Type FH Millipore tilter with a rated pore size of 0.5 microns.

The circular opening in the center of the fritted disc is initially filled with a wetting phase and then displaced by a nonwetting phase (air in most cases). The wetting phase drains through the pores of the fritted disc, leaving behind a layer of thin film adhering to the solid substrate. The pressure of the film phase relative to that of the nonwetting phase is controlled by the liquid level in the manometer. This procedure allows the direct measurement of the disjoining pressure. A minimum time of 1.5 hours was allowed for the film to reach equilibrium after each pressure adjustment.

\section{Results and discussion}

The equilibrium film thickness of $1 \times 10^{-4} \mathrm{M} \mathrm{KBr}, 1 \times 10^{-3} \mathrm{M} \mathrm{KBr}$, and $1 \times 10^{-4}$ $\mathrm{M} \mathrm{KCl}$ aqueous solutions on a borosilicate glass surface was measured as a function of the disjoining pressure at room temperature. The measurements were carried out using two different sizes of the fritted glass disc opening, 1 and $2 \mathrm{~mm}$ in diameter.

The typical air-solution interfacial profiles are shown in Figs. 2 and 3 for the disc opening diameters of 1 and $2 \mathrm{~mm}$, respectiveiv. The air-solution interfauial profile at each disjoining pressure is almost symmetric, with a minimum thickness at the center of the profile. The interfacial curvature is generally large at small disjoining pressures but becomes progressively smaller with an increase in the disjoining pressure. The central part of the film becomes almost flat at high disjoining pressures. The minimum film thickness decreases with an increase in the disjoining pressure as predicted by the DLVO theory.

The laser beam in the ellipsometer illuminates a cross section on the film surface of 25 by 75 microns. The normal spacing between thickness measurements along a profile is about 125 microns. Consequently, the detection of the interfacial curvature is not an artifact due to the finite laser beam size. The presence of a finite air-solution interfacial curvature implies that the disjoining pressure is not constant along the central portion of the thin film. This point seems to have been missed by previous investigators, who have assumed that the disjoining pressure is constant near the center. Since the abscissa in Figs. 2 and 3 is in microns whereas the ordinate is in angstroms, the actual interfacial curvature is much smaller than the apparent curvature shown. For a typical interfacial profile, the film thickness changes by about 100 angstroms over a length of 250 microns, which translates into an angle of inclination of only $0.002^{\circ}$. However, the variation of thickness over the length of the film is unmistakable. 
To reconcile the presence of thickness variations over the film length, we calculated the film thicknesses in the transition region based on the augmented Laplace equation with the assumption of a constant air-solution interfacial tension. Our preliminary results indicate that the thickness variations do not correspond to the transition region described by the augmerited Laplace equation. The modified Laplace equation predicts that the film should be flat over the greater portion of the opening and should not vary as revealed by the measurements. We are continuing to investigate the cause of this discrepancy.

The relationship between the equilibrium film thickness and the disjoining pressure is depicted in Figs. 4 to 9. The minimum film thickness of each interfacial profile is shown in these figures as the equilibrium film thickness, whereas the disjoining pressure is derived assuming that the air-solution interface near the region of minimum thickness:: planar. The disjoining pressure is expressed in centimeters of the film phase.

The disjoining pressure-thickness isotherms for $1 \times 10^{-4} \mathrm{M} \mathrm{KBr}$ and $1 \times 10^{-3} \mathrm{M}$ $\mathrm{KBr}$ solutions measured in a $1 \mathrm{~mm}$ diameter fritted disc opening are shown in Fig. 4 . The experiments are reproducible. The equilibrium film thickness at a given electrolyte concentration decreases monotonically with an increase of the disjoining pressure. The film thickness for $1 \times 10^{-4} \mathrm{M} \mathrm{KBr}$ solutions at low disjoining pressure is twice that of $1 \times 10^{-3} \mathrm{M} \mathrm{KBr}$ solutions. This behavior is in qualitative agreement with the fact that, when the ionic strength of the solution is increased, the thickness of the electrical double layer is reduced, and the film stabilizing effect due to the overlapping of the electrical double layer will become effective at a smaller separation between the air-sol stion and solution-substrate interfaces. The difference in the equilibrium thicknesses at the two concentrations becomes smaller at higher disjoining pressure, and they both fall in the range of 150 to 200 angstroms at the pressure of 20 to $40 \mathrm{~cm}$ of the film phase.

The same experiments were repeated using a fritted disc with a $2 \mathrm{~mm}$ diameter opening. The isotherms are shown in Fig. 5. The difference between the isotherms of the two KBr concentrations seen in Fig. 4 is no longer observed except for the two points at 750 to 800 angstroms for $1 \times 10^{-4} \mathrm{M} \mathrm{KBr}$. A consistent trend is that films that are thicker tend to be more sensitive to the diameter of the circular opening and, when the opening is as large as $2 \mathrm{~mm}$, only very thick films deviate from those in the $1 \mathrm{~mm}$ opening. These deviations occur at very small disjoining pressures and may be less reliable than values for tilms that are thinner.

The effect of the size of the fritted disc opening, or rather the lateral-film dimension, can be seen easily in Fig. 6 . The isotherms measured in a $1 \mathrm{~mm}$ diameter opening. represented by the solid symbols, are clearly different from those measured in a $2 \mathrm{~mm}$ 
diameter opening. represented by the open symbols. However, the effect of the lateral film dimension for $1 \times 10^{-3} \mathrm{M} \mathrm{KBr}$ either diminishes or becomes too small to be detected within our experimental accuracy, as depicted in Fig. 7. We do not yet have an explartation of why the lateral film dimersion has an apparent effect on the equilibrium film thickness.

The isotherms of $1 \times 10^{-4} \mathrm{M} \mathrm{KBr}$ and $1 \times 10^{-4} \mathrm{M} \mathrm{KCl}$ in 1 and $2 \mathrm{~mm}$ openings are compared in Figs. 8 and 9, respectively. The isotherms of the two different electrolytes at a given concentration are identical within experimental accuacies, except for the two points at 750 to 800 angstroms in Fig. 9. This observation implies that the disjoining pressurethickness isotherms for simple electrolyies may not be chemical specific.

\section{Foams for Mobility Control Stability of Liquid Collars at Pore Throats}

\section{Introduction}

The mechanism by which foam forms in porous media is now thought to be due to snap-off at pore throats which is often calculated assuming the geometry of the liquid film at the pore throat precisely corresponds to the geometry of the pore throat. This effectively neglects the thickness of the liquid collar. This method of calculation is called the Roof criterion (Roof, 1970). This can, however, be a very poor approximation and furthermore does not take into account the contact angle. We have been studying the stability of equilibrium interface shapes resulting from the interaction of two i. -miscible fluid phases with a solid to improve the approximation now used for predicting snap-off. This involves minimizing the free energy

$$
d F=\left(P_{\beta}-P_{\alpha}\right) d V_{\alpha}+\gamma_{\alpha \beta} d A_{\alpha \beta}-\gamma_{\alpha \beta} \cos \theta d A_{\alpha \gamma}
$$

of a three-phase system where the $\alpha$-phase is the wetting fluid, the $\beta$-phase is the nonwetting fluid, and the $\gamma$-phase is the solid. The contact angle is $\theta$ and the interfacial tension is $\gamma_{\alpha \beta}$. Requiring the first variation of the free energy, $d F$, to vanish implies that the equilibrium interface shapes must s...sfy the Laplace and Young equations.

When the solid is restricted to surfaces with axial symmetry, the solutions to the Laplace equation must also have axial symmetry. The meridional curves for these surfaces are unduloids and nodoids:

$$
y(\psi \cdot \phi . H)=\frac{\sqrt{1-\sin ^{2} \phi \sin ^{2} y}}{H(1+\cos \phi)}
$$




$$
x(\psi, \mathcal{O}, \mathrm{D}, \mathrm{H})=\frac{E(\psi \cdot \sin \phi)+\cos \phi \mathrm{F}(\psi, \sin \phi)-\mathrm{D}}{\mathrm{H}(1+\cos \phi)}
$$

where $\mathrm{H}$ is the absolute value of the mean curvature, $\psi$ is the curve parameter. and $\phi$ and $\mathrm{D}$ are integration constants. The functions $F(\psi \cdot \sin 0)$ and $E(\psi \cdot \sin 0)$ are Legendre's incomplete elliptic integrals of the first and second kind. The curves are unduloids for 0 in the interval $(0, \pi / 2)$ and nodoids for $\phi$ in the interval $(\pi / 2, \pi)$.

The analysis of the interface shapes proceeds in two steps: (1) find the equilibrium interface shapes and (2) test for their stability. The equilibrium interface shapes are found for a given solid shape by requiring the interface to contact the solid with a specified contact angle. The meridional curve for the solid is presumed to be

$$
g(x)=\frac{\lambda \mu}{2}\left[(\delta+1)+(\delta-1) \cos \left[\frac{\pi \xi}{\lambda}\right]\right]
$$

When the boundary conditions are solved, the result is the endpoint of a segment of an equilibrium curve. Three typical solutions are shown in Fig. 10 for a contact angle of $\theta=$ $0^{\circ}$ and a solid with parameters $\lambda=1, \mu=1$, and $\delta=0.1$. The solid $\gamma$-phase is above the solid meridion, and the wetting $\alpha$-phase is in the region below the solid meridion and above the interface meridians. The meridians are revolved around the $x$-axis to generate the surfaces. As the volume of the $\alpha$-phase increases, the $\alpha-\beta$ interface passes through the shapes whose boundary points are denoted by $c, b$, and a. At the boundary point a, the interface is no longer stable and snap-off of the $\alpha$-phase begins. In Fig. 11, the mean curvature versus the wetting phase saturation is shown. As the capillary pressure decreases, the $\alpha$ - $\beta$ interface passes through points $c, b$, and a and then snap-off begins. The Roof snap-off criterion is siown for comparison with the point a.

It would be convenient if a series of boundary condition solutions could be characterized by the endpoints rather than the series of equilibrium segments. We have Geveloped two complementary methods for representing the endpoints: (1) the phase space plot and (2) the arc length plot. It turns out that the phase configuration of a particular equilibrium interface with respect to the solid can also be characterized by examining only the contact point. The configuration test presented here indicates whether a given intertace is inside or outside of the solid and whether the interface bounds the wetting $\alpha$-phase or the 
nonwetting $\beta$-phase. Finally, the stability test for a given equilibrium interface shape is given.

\section{Phase space plot}

This method of plotting the boundary points is derived from the phase space of the Hamiltonian form of the free energy. It involves plotting $\cos \phi$ versus $y^{*}(\psi, 0)=$ $\sqrt{1+\sin ^{2} \phi \sin ^{2} \psi}$ for each endpoint $(y, f)$ as shown in Fig. 12. As a given unduloid or nodoid is traversed, a point $\psi$ on the curve oscillates along a line of constant $\phi$ between the minimum amplitude $\mathrm{y}^{*}=\cos \phi$ and the maximum amplitude $\mathrm{y}^{*}=1$.

For example, for $\phi=90^{\circ}$, the curve is a series of semicircles with cusps on the $x$-axis. This equilibrium curve shows up on Fig. 12 as the line $\cos \phi=0$ with minimum amplitude 0 and maximum amplitude 1 . If the endpoint happens to be $y^{*}=0.5$, then the portion of the $y^{*}$ axis to the right of the endpoint corresponds to the circular segments tangent to $y^{*}=1$ and the portion to the left corresponds to the circular segments with cusps at $\mathrm{y}^{*}=0$.

\section{Arc length plot}

The second plot is derived from the arc length. The change in arc length, $s$, is proportional to the change in $\psi$ :

$$
\mathrm{Hds}=\mathrm{d} \psi
$$

If the unit of arc length is one-half of a wavelength, then the function

$$
\mathrm{P}^{*}(\psi)=\frac{\psi-\psi_{\text {sym }}}{\frac{\pi}{2}}=\frac{H\left(s-s_{s y m}\right)}{\frac{\pi}{2}}
$$

is a measure of the distance of the point $\psi$ from the symmetry point $\psi_{\text {sym }}$. For the curves in Eq. (2) we define the symmetry points as

$$
\psi_{\text {sym }}=\left\{\begin{array}{l}
0, \text { symmetry about a maximum } \\
\pi \\
2, \text { symmetry about a minimum }
\end{array}\right.
$$


Clearly then, as a given unduloid or nodoid is traversed for $1 / 2$ of a wavelength on either side of a symmetry point. $\mathrm{P}^{*}$ varies from -1 to 1 along a line of constant $\phi$. Figure 13 shows this plot for the same data as shown in Fig. 12.

\section{Configuration test}

We need to know if a given boundary condition solution refers to an interface which is inside or outside of the solid and whether the interface bounds the wetting $\alpha$-phase or the nonwetting $\beta$-phase. The four basic configurations are shown in Fig. 14. The meridional curves for the interface and solid are denoted by $f$ and $g$. The $x$-axis is the axis of revolution.

The problem is approached by first defining a local coordinate system $v_{x}$ and $v_{y}$ relative to $g$ at the boundary point. $v_{y}$ is the "outward" pointing normal of $g$ and $v_{x}$ is the tangent pointing towards the symmetry point of the solid. Next, a local coordinate $w_{x}, w_{y}$ is defined relative to $f$ at the boundary point. The tangent vector, $w_{x}$, is defined to point towards the symmetry point of $f$. The normal vector $w_{y}$ points from the $\beta$-phase to the $\alpha$.phase. The angle $\theta$ between $w_{x}$ and $v_{x}$ is the crucial quantity for this test. It turns out that in $v_{x}, v_{y}$ coordinates,

$$
\begin{aligned}
& \cos \theta=\operatorname{sign}(-x) \operatorname{sign}\left(\psi_{\text {sym }}-\psi\right)\left\{\frac{x^{\prime}-y^{\prime} g^{\prime}}{\sqrt{1+g^{\prime 2}} \sqrt{x^{\prime 2}+y^{\prime 2}}}\right\} \\
& \sin \theta=\operatorname{sign}\left(\psi_{\text {sym }}-\psi\right)\left\{\frac{-x^{\prime} g^{\prime}+y^{\prime}}{\sqrt{1+g^{\prime 2}} \sqrt{x^{\prime 2}+y^{\prime 2}}}\right\}
\end{aligned}
$$

where the sign function is either +1 or -1 , depending on the sign of its argument. The primes denote differentiation with respect to $\psi$ for $\mathrm{x}(\psi), \mathrm{y}(\psi)$ and with respect to $\mathrm{x}$ for $\mathrm{g}(\mathrm{x})$.

The tests for inside/outside and $\alpha$-bound/ $\beta$-bound now become apparent. If $\sin \theta>0$, then the interface is outside and if $\sin \theta<0$, the interface is inside. If $\cos \theta>0$, then the $\alpha$-phase is bound and if $\cos \theta<0$, then the $\beta$-phase is bound.

The relationship between the phase normal $w_{y}$, which points from the $\beta$-phase to the $\alpha$-phase, and the outward normal is crucial for determining the correct sign of the capillary pressure, $2 \gamma_{\alpha \beta} H=P_{\beta}-P_{\alpha}$. If the outward normal of Eq. (2) is defined as 


$$
n=\frac{-y^{\prime} i+x^{\prime} j}{\sqrt{x^{2}+y^{2}}}
$$

and the mean curvature is

$$
2 H=\Omega\left\{-\frac{x^{\prime} y^{\prime \prime}-x^{\prime \prime} y^{\prime}}{\left(x^{\prime 2}+y^{\prime 2}\right)^{3 / 2}}+\frac{x^{\prime}}{y \sqrt{x^{2}+y^{\prime 2}}}\right\}
$$

where

$$
\Omega=\left\{\begin{array}{l}
+1, \text { outward normal } \\
-1, \text { inward normal }
\end{array}\right.
$$

The function $\Omega$ can be computed from

$$
\Omega=\mathbf{n} \sum \mathbf{w}_{\mathrm{y}}=-\operatorname{sign}(\sin \theta \cos \theta) \operatorname{sign}(-\mathrm{x}) \operatorname{sign}\left(\psi_{\mathrm{sym}}-\psi\right)
$$

\section{Variable endpoint stability test}

The discussion up to this point is focused on properties of equilibrium interface shapes. The central question in our investigations is the stability of these shapes. Whereas equilibrium interface shapes occur when the first variation, $\mathrm{dF}$, of the free energy vanishes, stability of an equilibrium shape requires that the second variation, $\mathrm{d}^{2} \mathrm{~F}$, be positive.

Theorems from the calculus of variations show that an equilibrium interface shape with endpoints varying along a solid is stable if it satisfies Legendre's condition and an analogue to Jacobi's condition. Legendre's condition implies a stable shape: (1) may not contact the $x$-axis and (2) may not contain vertical tangents. The analogue to Jacobi's condition for variable endpoints implies that an equilibrium interface shape satisfying the boundary conditions and Legendre's condition is stable if it contains no focal points of the solid. This means that the function

$$
D(\psi)=\operatorname{det}\left(\begin{array}{ccccc}
x_{\phi} & x_{D} & x_{\psi} & 0 & 0 \\
y_{\phi} & 0 & y_{\psi} & 0 & 0 \\
x_{a \phi} & x_{a D} & 0 & x_{a \psi} & -1 \\
y_{a \phi} & 0 & 0 & y_{a \psi} & -g_{a x} \\
T_{a \phi} & T_{a D} & 0 & T_{a \psi} & T_{a x}
\end{array}\right)
$$


has no zeros for $\psi$ between the endpoints of the equilibrium interface. The functions $x, y$, and $g$ are given by Eqs. (2) and (3). The function $T$ is the transversality condition

$$
T(\psi, \phi, H . D, x, \theta)=\cos \theta \sqrt{x_{\psi}^{2}+y_{\psi}^{2}} \sqrt{1+g_{x}^{2}}-x_{\psi}-y_{\psi} g_{x}=0
$$

The subscripts $\Phi, \mathrm{D}, \psi$, and $\mathrm{x}$ refer to differentiation with respect to those variables. The subscript a means the function is evaluated at the boundary point.

\section{Conclusions}

Extensive calculations based on different pore geometries and contact angles have been completed and the results are being catalogued. Important are the saturations in a single pore as a function of the capillary pressure. There are also two important points of instability and both are shown in Figs. 10 and 11. At high capillary pressure the saturation is small and the liquid collar is simply a thin wetting film. As the capillary pressure is decreased, the collar increases in thickness and at some point growth is spontaneous. The liquid collar then progresses through a sequence of shapes corresponding to a progression of increasing capillary pressures until it arrives at a state unstable to volume conserving perturbations. This is denoted in Fig. 1 as the limiting stable surface. It is at this $p$ r int that snap-off occurs. This analysis assumes that liquid flow into pore throats is restricted and the configuration of the liquid collar even though growing is dominated by capillary forces. If this approximation is valid, then our analysis provides a measure of the rate of lamella formation at a pore generating site.

\section{References}

Roof. J.G.: "Snap-Off of Oil Droplets in Water-Wet Pores," Soc. Pet. Eng. J. (1970) 10, No. 1,85 .

\section{Nomenclature}

$\begin{array}{ll}\mathrm{A}_{\alpha \beta} & =\text { interfacial area } \\ \mathrm{A}_{\alpha \gamma} & =\text { wetted area } \\ \mathrm{D} & \text { integration constant of Eq. (2) } \\ \mathrm{E}_{(\psi, \sin O)}=\text { Legendre incomplete elliptic integral of the second kind } \\ \mathrm{f} & =\text { meridional curve of the interface } \\ \mathrm{F}(\psi, \sin 0) & =\text { Legendre incomplete elliptic integral of the first kind }\end{array}$




\begin{tabular}{|c|c|c|}
\hline$F$ & $=$ & free energy \\
\hline$g$ & $=$ & meridional curve of the solid \\
\hline $\mathrm{H}$ & $=$ & mean curvature \\
\hline $\mathbf{n}$ & $=$ & the outward normal of Eq. (2) \\
\hline $\mathrm{P}^{*}$ & $=$ & normalized arc length \\
\hline$P_{\alpha}$ & $=$ & bulk $\alpha$-phase pressure \\
\hline$P_{\beta}$ & $=$ & bulk $\beta$-phase pressure \\
\hline s & $=$ & arc length \\
\hline $\mathrm{T}$ & $=$ & transversality condition \\
\hline$v_{x}$ & $=$ & the tangent to $g$ at a boundary point \\
\hline $\mathbf{v}_{\mathrm{y}}$ & $=$ & the outward normal to $g$ at a boundary point \\
\hline $\mathrm{V}_{\alpha}$ & $=$ & $\alpha$-phase volume \\
\hline $\mathbf{w}_{\mathrm{x}}$ & $=$ & the tangent to f at a boundary point \\
\hline $\mathbf{w}_{y}$ & $=$ & the phase oriented normal to $f$ at a boundary point \\
\hline$y^{*}$ & $=$ & normalized radial coordinate of Eq. (2) \\
\hline$\gamma_{\alpha \beta}$ & $=$ & interfacial tension \\
\hline$\delta$ & $=$ & solid surface parameter \\
\hline$\theta$ & $=$ & contact angle \\
\hline$\lambda$ & $=$ & solid surface parameter \\
\hline$\mu$ & $=$ & solid surface parameter \\
\hline$\phi$ & $=$ & integration constant of Eq. (2) \\
\hline$\psi$ & $=$ & curve parameter of Eq. (2) \\
\hline$\Psi_{\text {Sy m }}$ & $=$ & $\psi$ at a maximum or minimum of Eq. (2) \\
\hline$\Omega$ & $=$ & the orientation function for the normal of Eq. (2) \\
\hline
\end{tabular}




\section{List of Figures}

Figure

1 The schematic diagram of the microcell assembly and the manometer

2 The film thickness of $1 \times 10^{-4} \mathrm{M} \mathrm{KCl}$ as a function of the lateral position of the sample stage at various disjoining pressures, $-\mathrm{H}$, expressed in $\mathrm{cm}$ of $\mathrm{KCl}$ solution. The fritted disc opening is $1 \mathrm{~mm}$ diameter

3 The film thickness of $1 \times 10^{-4} \mathrm{M} \mathrm{KBr}$ as a function of the lateral position of the sample stage at various disjoining pressures, $-\mathrm{H}$, expressed in $\mathrm{cm}$ of $\mathrm{KBr}$ solution. The fritted disc opening is $2 \mathrm{~mm}$ diameter

4 The disjoining pressures as functions of the equilibrium film thickness for $1 \mathrm{x}$ $10^{-4} \mathrm{M} \mathrm{KBr}$ and $1 \times 10^{-3} \mathrm{M} \mathrm{KBr}$. The fritted disc opening is $1 \mathrm{~mm}$ diameter

5 The disjoining pressures as functions of the equilibrium film thickness for $1 \mathrm{x}$ $10^{-4} \mathrm{M} \mathrm{KBr}$ and $1 \times 10-3 \mathrm{M} \mathrm{KBr}$. The fritted disc opening is $2 \mathrm{~mm}$ diameter

6 The disjoining pressures of $1 \times 10^{-4} \mathrm{M} \mathrm{KBr}$ as functions of the equilibrium film thickness for the fritted disc openings of 1 and $2 \mathrm{~mm}$ in diameter

7 The disjoining pressures of $1 \times 10^{-3} \mathrm{M} \mathrm{KBr}$ as functions of the equilibrium film thickness for the fritted disc openings of 1 and $2 \mathrm{~mm}$ in diameter

The disjoining pressures of $1 \times 10^{-4} \mathrm{M} \mathrm{KBr}$ and $1 \times 10^{-4} \mathrm{M} \mathrm{KCl}$ as functions of the equilibrium film thickness for the fritted disc opening of $1 \mathrm{~mm}$ in diameter

9 The disjoining pressures of $1 \times 10^{-4} \mathrm{M} \mathrm{KBr}$ and $1 \times 10^{-4} \mathrm{M} \mathrm{KCl}$ as functions of the equilibrium film thickness for the fritted disc opening of $2 \mathrm{~mm}$ in diameter

10 Stable equilibrium phase interfaces bounding the wetting $\alpha$-phase and inside the solid. The boundary points are indicated by $a, b$, and $c$. The contact angle is $\theta$ $=0^{\circ}$ and the solid surface parameters are $\lambda=1, \mu=1$, and $\delta=0.1$

11 Mean curvature $2 \mathrm{H}=\left(\mathrm{P}_{\alpha}-\mathrm{P}_{\beta}\right) / \gamma \alpha \beta$ versus wetting phase saturation for the same conditions as in Fig. 1

12 Phase space plot of the boundary points for the stable interfaces with contact angle $\theta=0^{\circ}$. The solid parameters are the same as in Fig. 1

13 Arc length plot of the boundary points for the stable interfaces with contact angle $\theta=0^{\circ}$. The solid parameters are the same as in Fig. 1

14 The four-phase configurations showing the local coordinate systems 


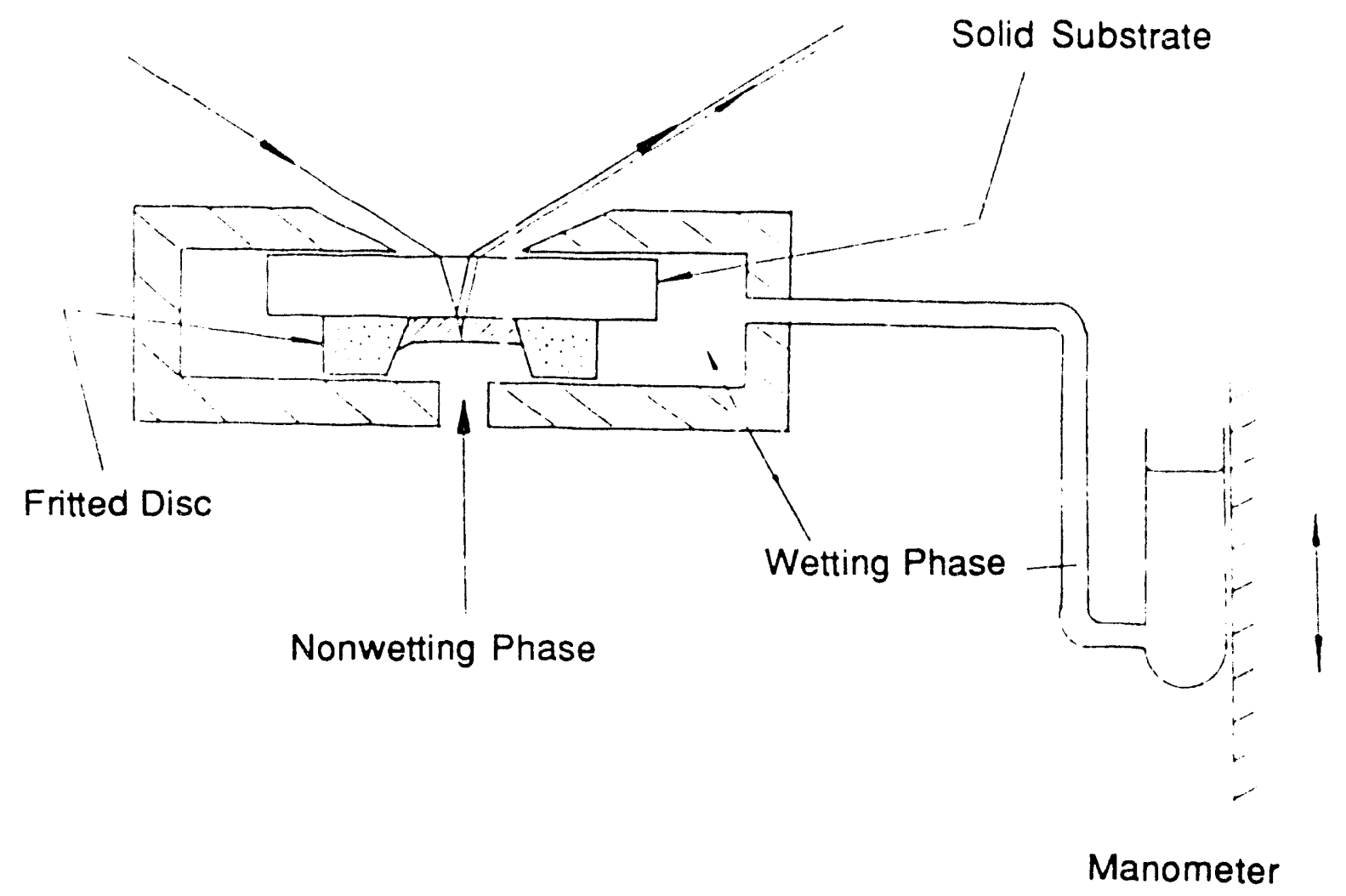

Figure 1. Thie schematic diagram of the microcell assembly and the manometer. 


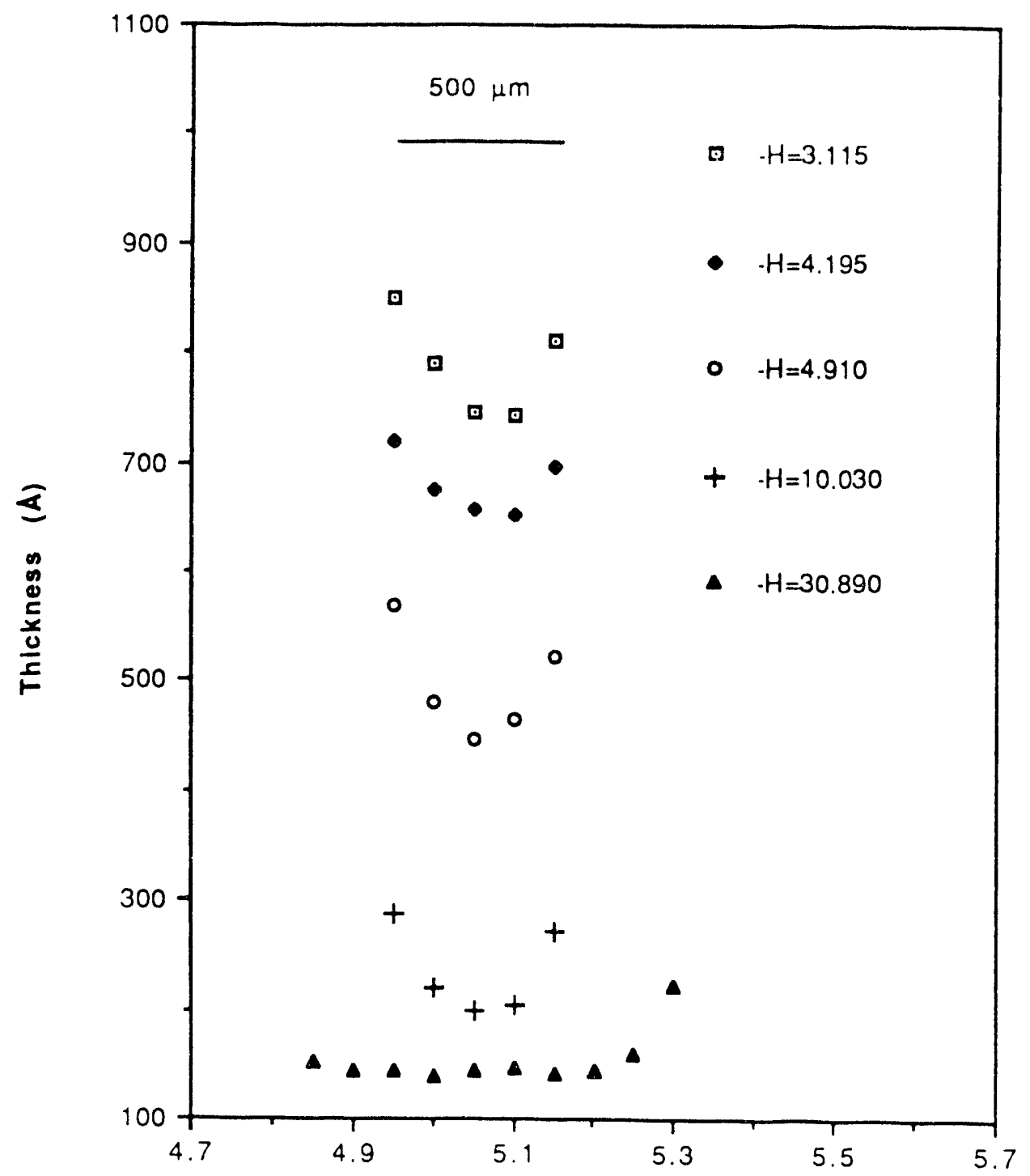

$X$-stage Value

Figure 2. The film thickness of $1 \times 10^{-4} \mathrm{M} \mathrm{KCl}$ as a function of the lateral position of the sample stage at various disjoining pressures, $-\mathrm{H}$, expressed in $\mathrm{cm}$ of $\mathrm{KCl}$ solution. The fritted disc opening is $1 \mathrm{~mm}$ diameter. 


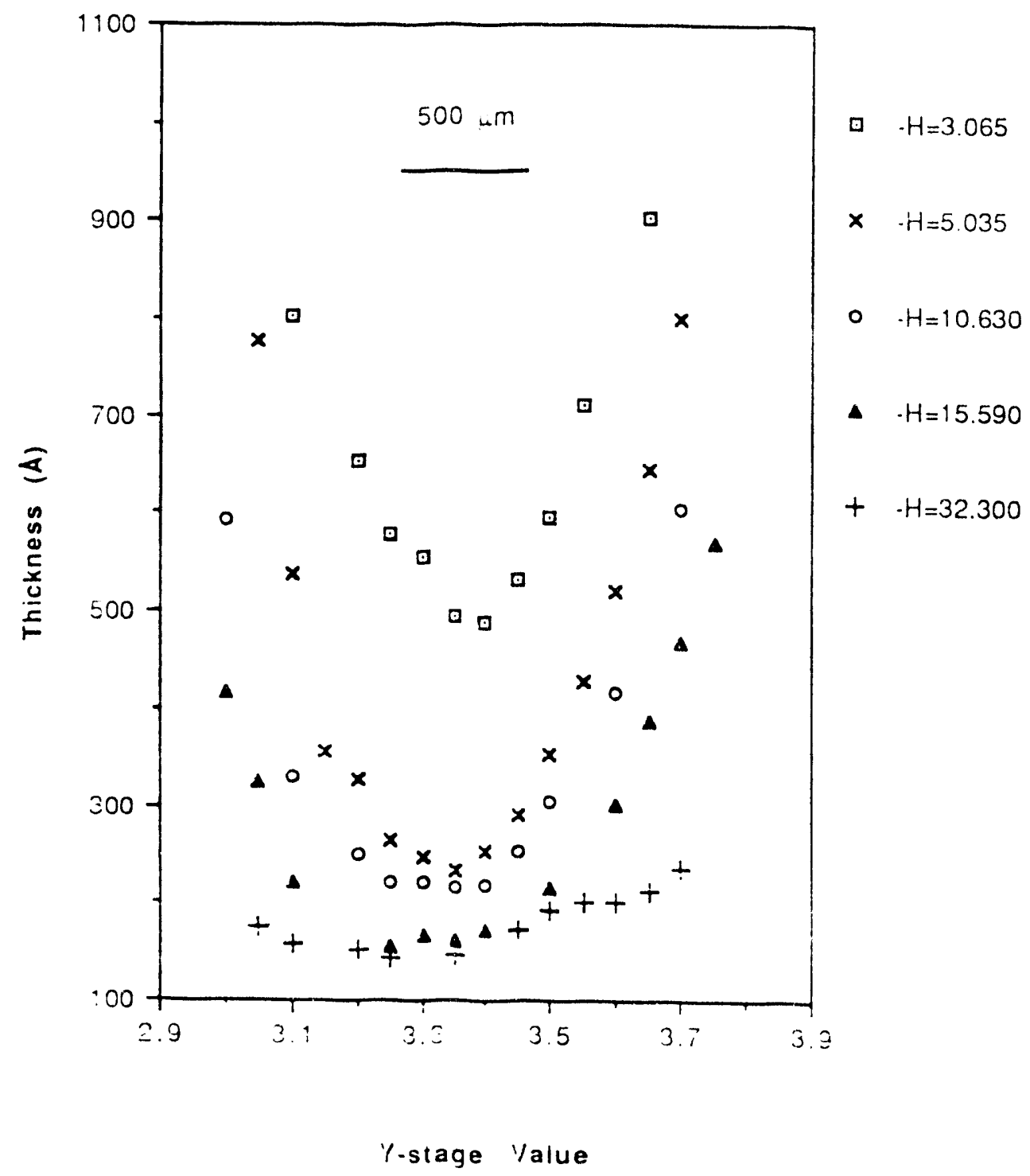

Tigure 3. The tilm thickness of $1 \times 10^{-4} \mathrm{M} \mathrm{KBr}$ as a function of the lateral position of the sample stage at various disjoining pressures. $-H$. expressed in $\mathrm{cm}$ of $\mathrm{KBr}$ solution. The fritted disc opening is 2 mm diameter. 


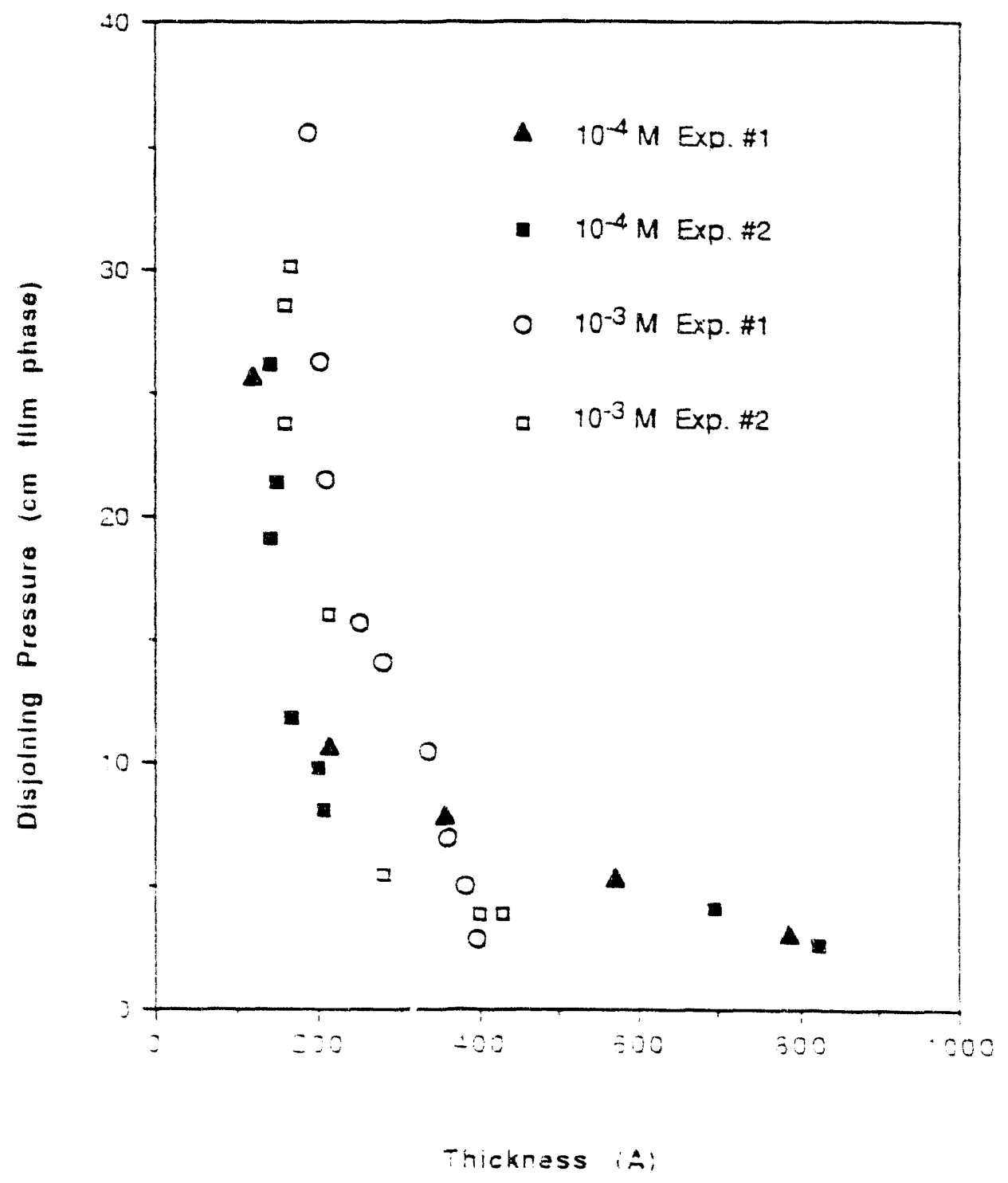

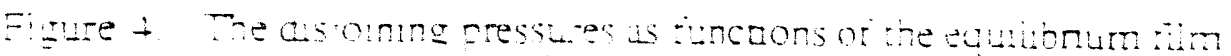

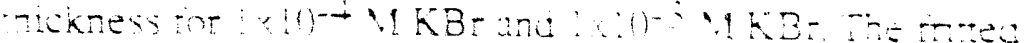

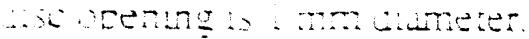




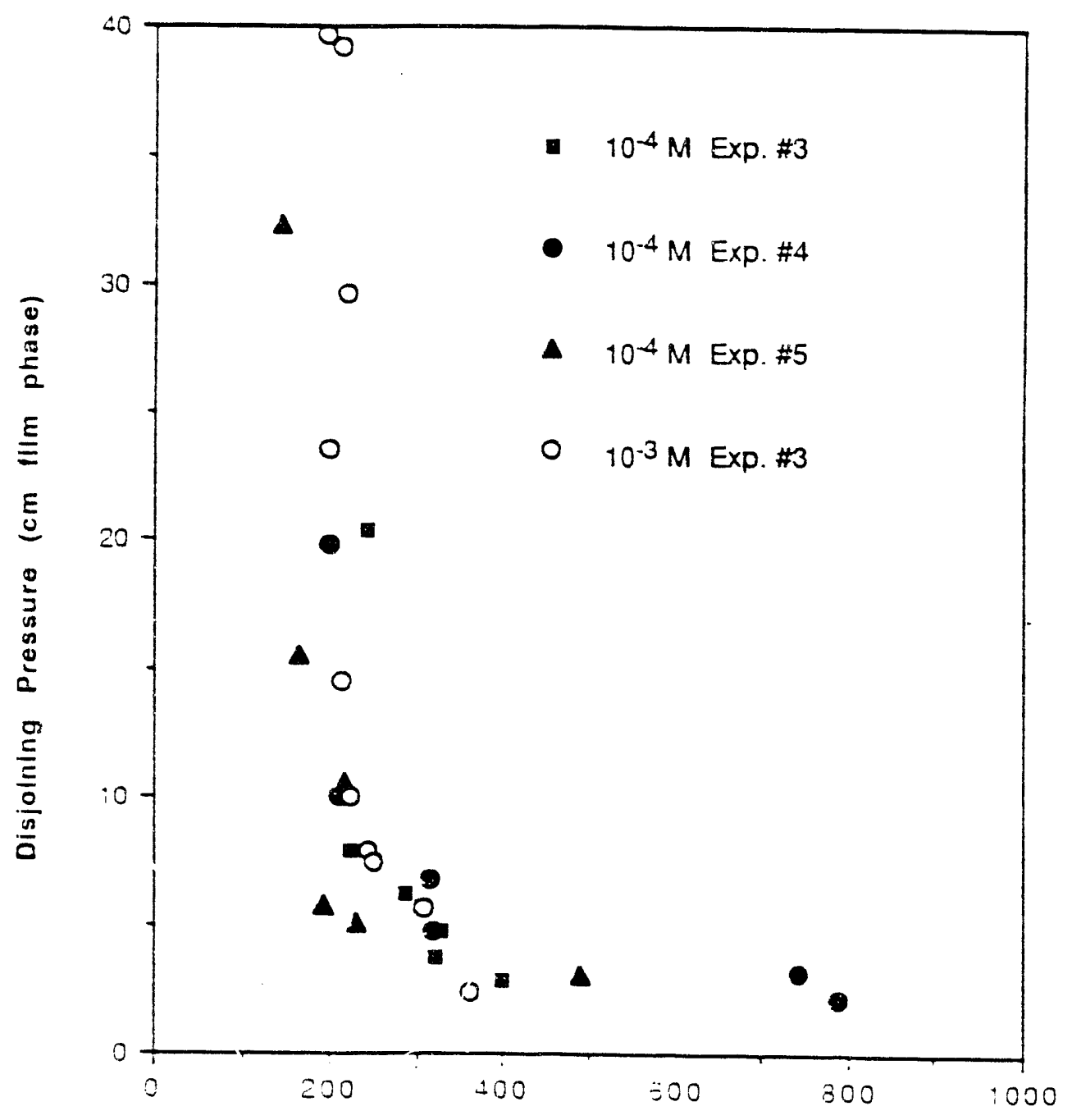

Thickness (A)

Figure 5. The disjoining pressures as funcaons of the equiliorium film inickness for $1 \times 10^{-+} \mathrm{M} \mathrm{KBr}$ and $1 \times 10^{-3} \mathrm{MKBr}$. The inimed isc opening is $2 \mathrm{~mm}$ diameter. 


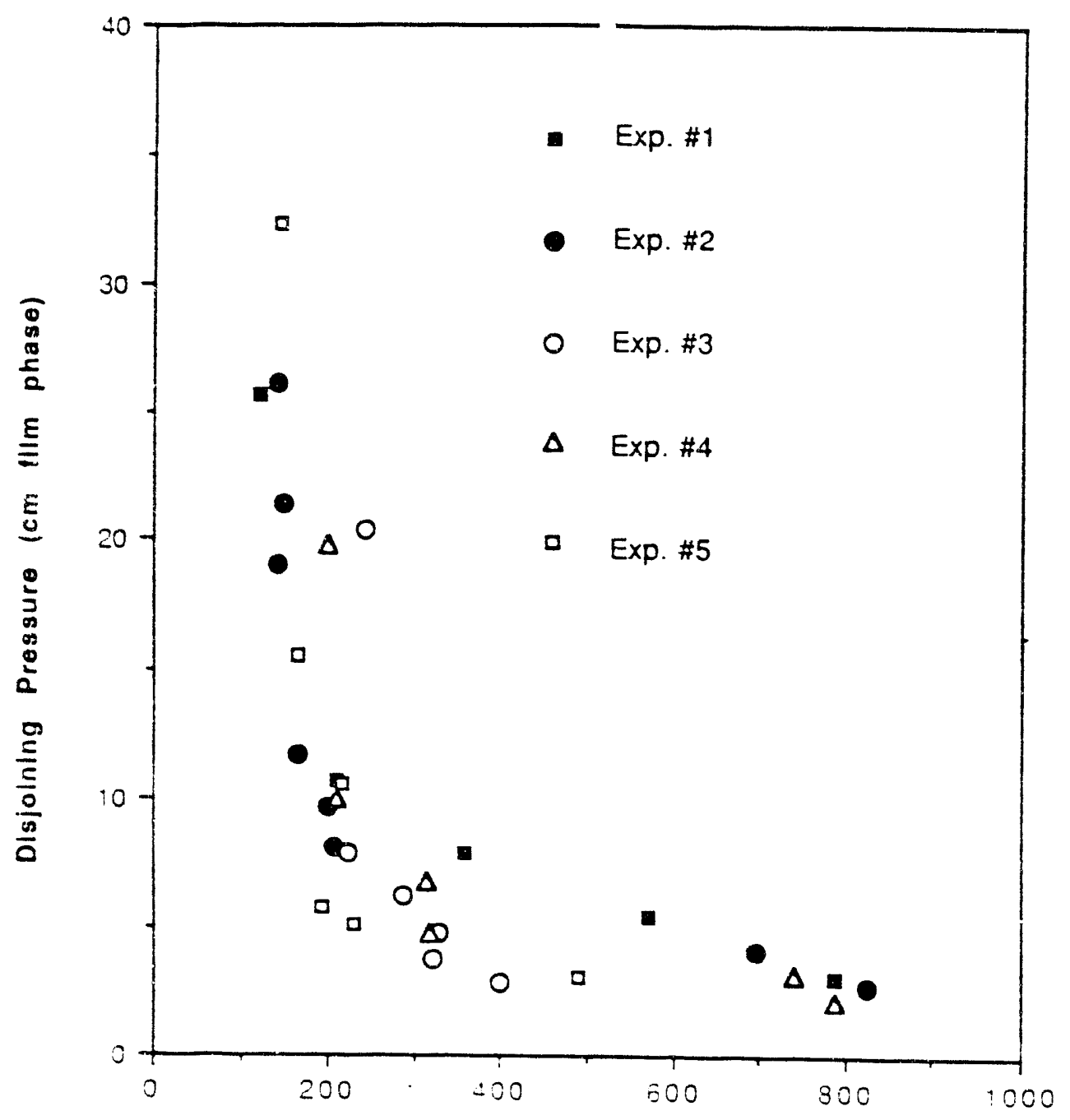

Thickness (A)

Figure 0 . The disjoining pressures of $1 \times 10^{-4} \mathrm{M} \mathrm{KBr}$ as funcions of the equilibrium film thickness for the frimed disc openings of 1 and $2 \mathrm{~mm}$ in diameter. The data shown correspond to those in Figures 4 and 5. 


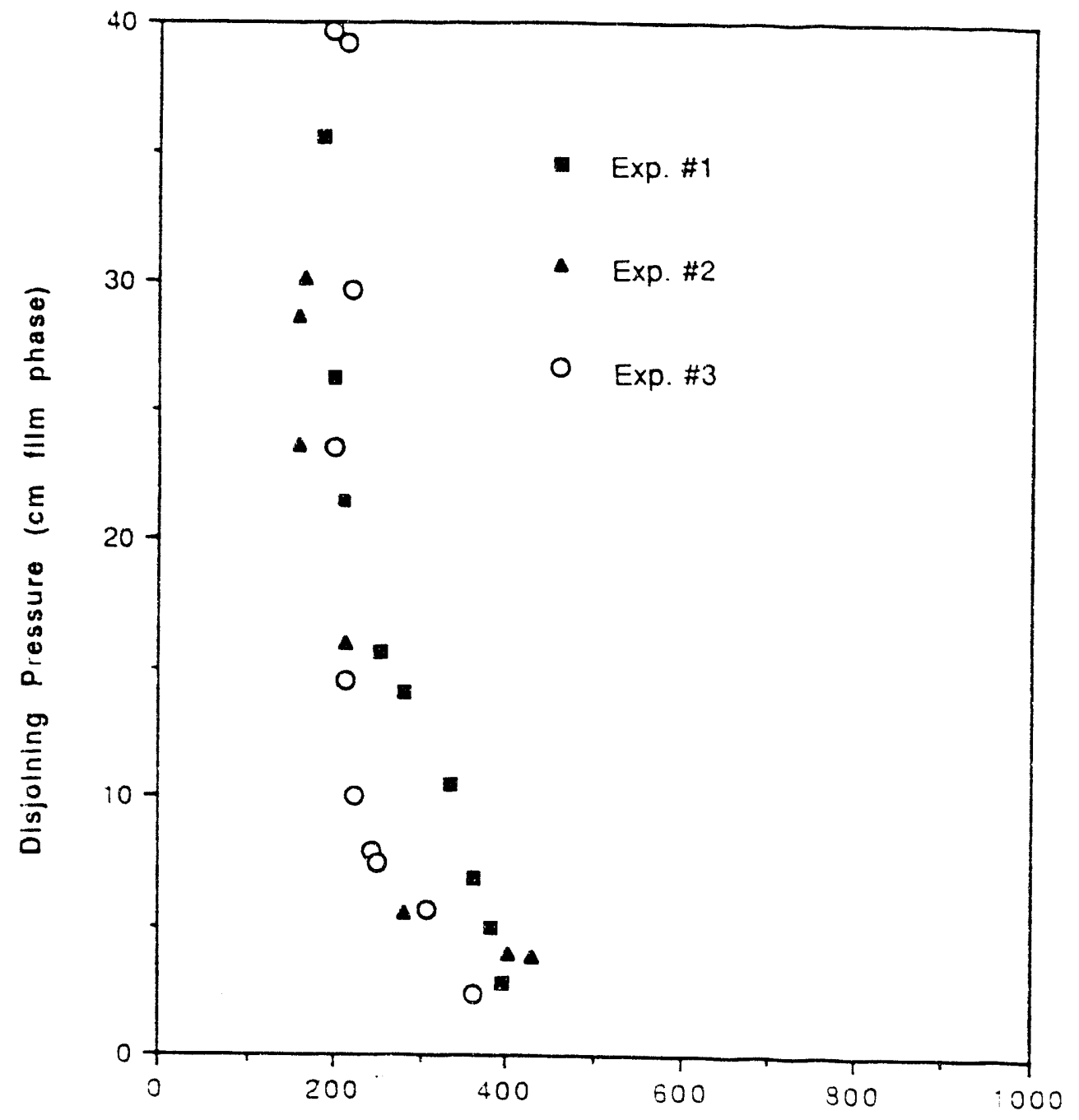

Thickness (A)

Figure 7 . The disjoining pressures of $1 \times 10^{-3} \mathrm{M} \mathrm{KBr}$ as funcions of the equilibrium film thickness for the fritted disc openings of 1 and $2 \mathrm{~mm}$ in diameter. The data shown correspond to those in Figures 4 and 5. 


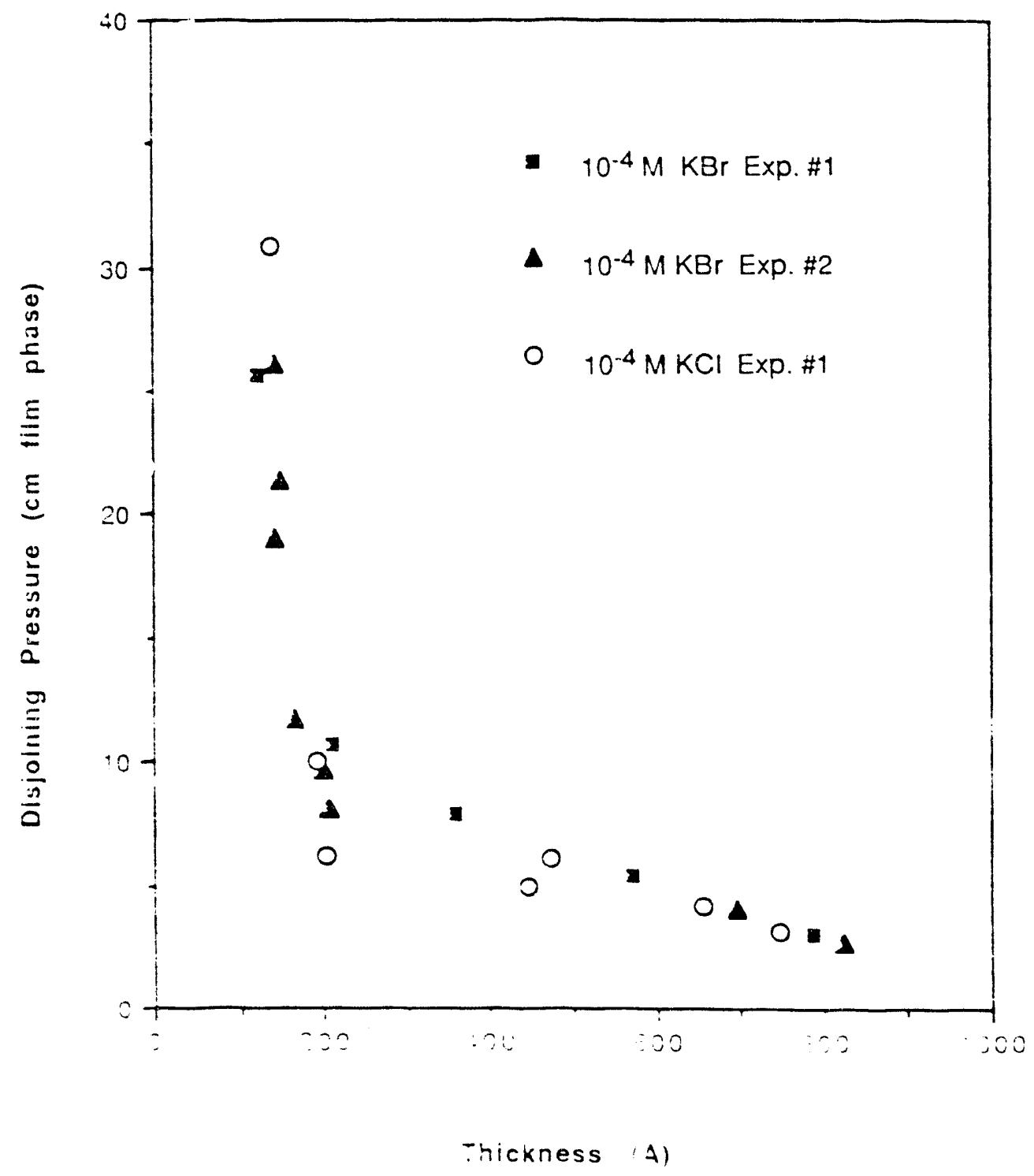

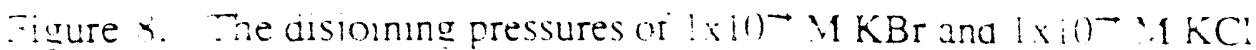

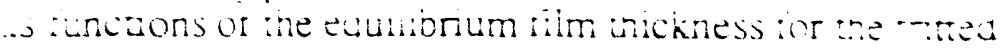
ase vorning of $1 \mathrm{~mm}$ in diameter. 


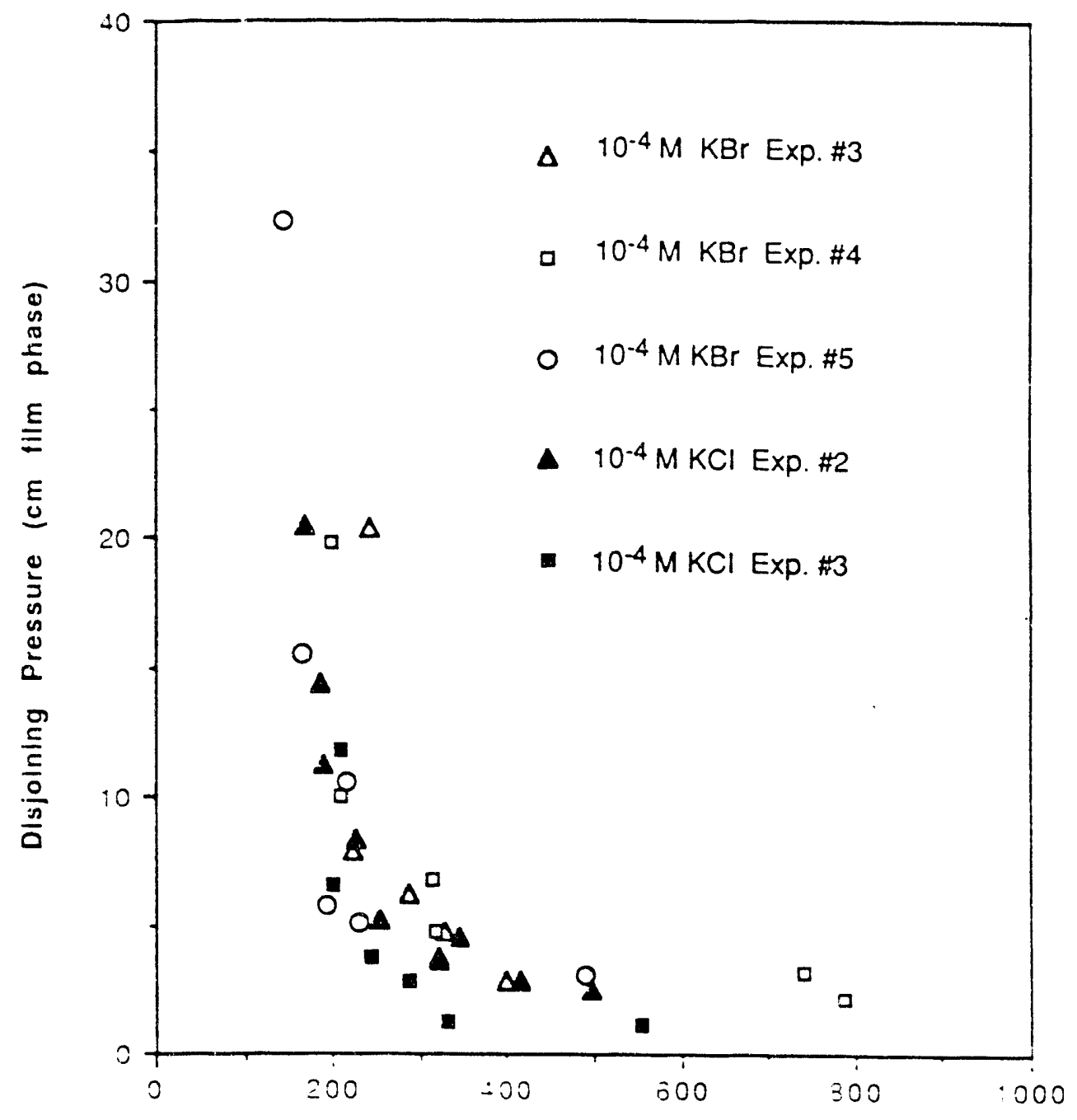

Thickness (A)

Figure 9 . The disjoining pressures of $1 \times 10^{-} \mathrm{M} \mathrm{KBr}$ and $1 \times 10^{-} \mathrm{M} \mathrm{KCl}$ is tuncuons of the equilibrium tilm thickness for the rritted jisc opening of $2 \mathrm{~mm}$ in diameter. 


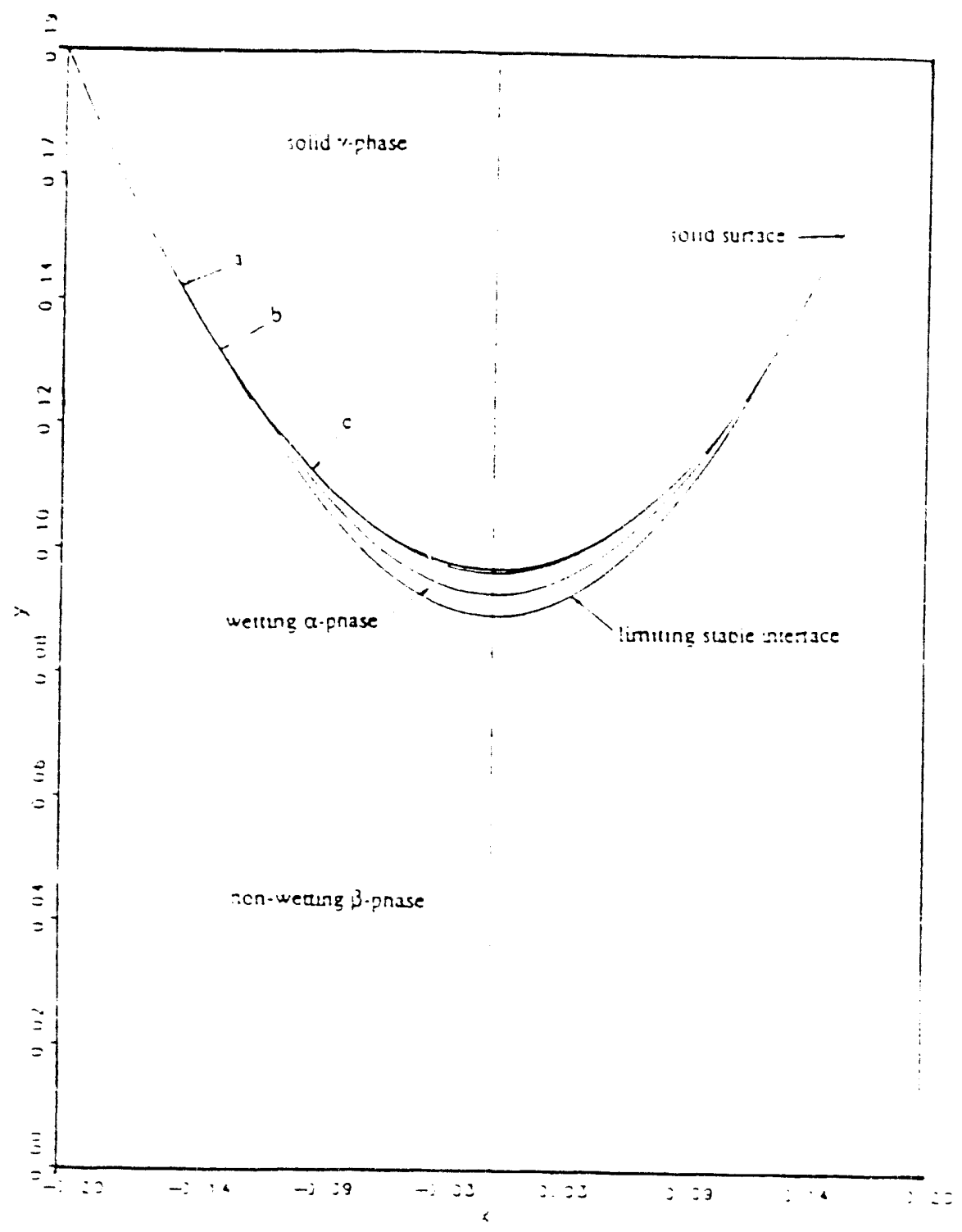

Figure 10. Stable equiibrium pnase interiaces bounding the wetung $\alpha$-phase and inside the solid. The boundary points are :naicated by a. 0 . and $c$. The contact angle is $\theta=11^{\circ}$ and the solid suriace parameters are $\dot{\lambda}=i, \mu=i$. and $j \dot{j}$ ). 1 . 


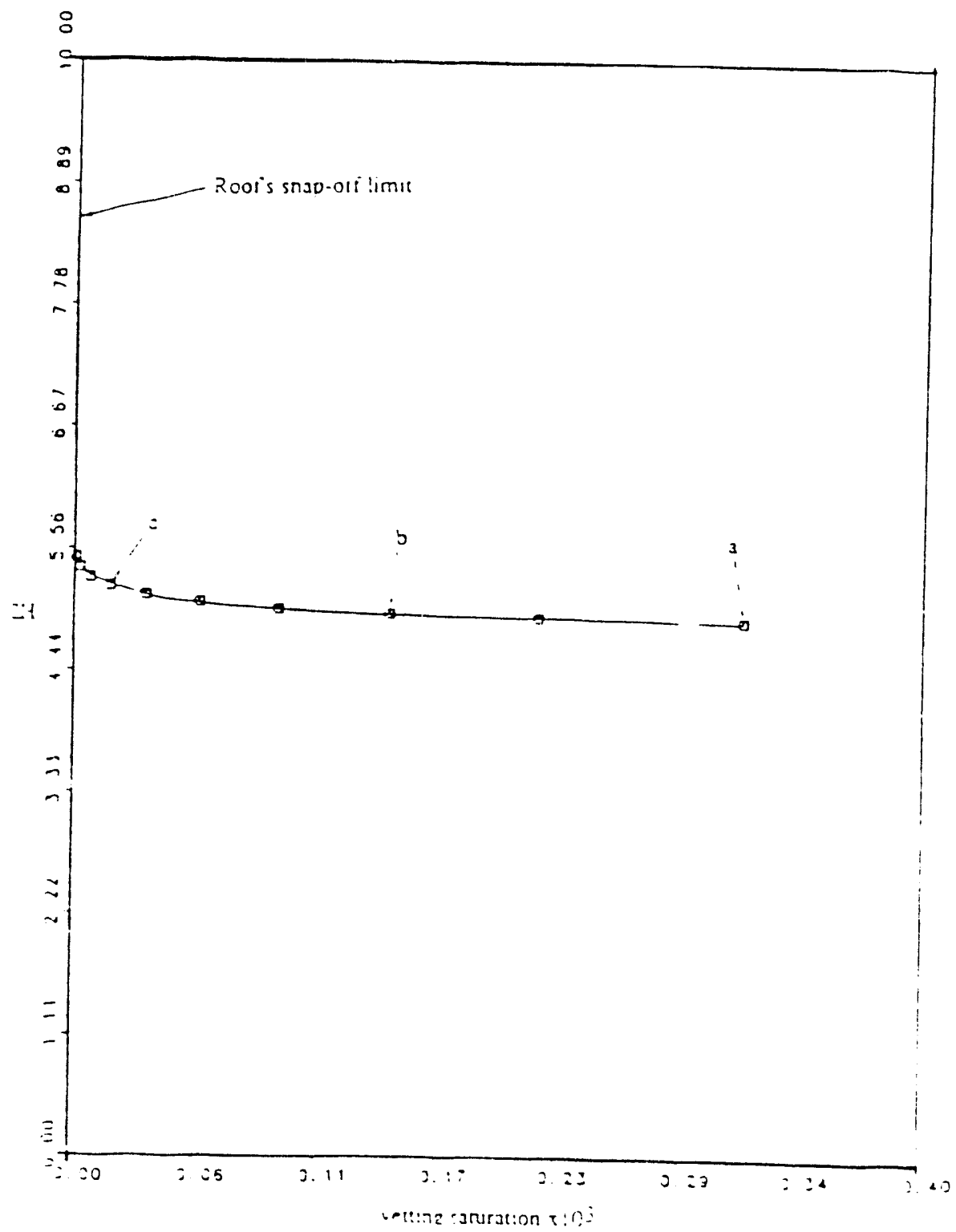

Figure 11. Mean curvature $2 \mathrm{H}=\left(\mathrm{P}_{\gamma_{x}}-\mathrm{P}_{3}\right) /_{a x 3}$ versus wetting phase saturation for the same conditons as in Figure 1. 


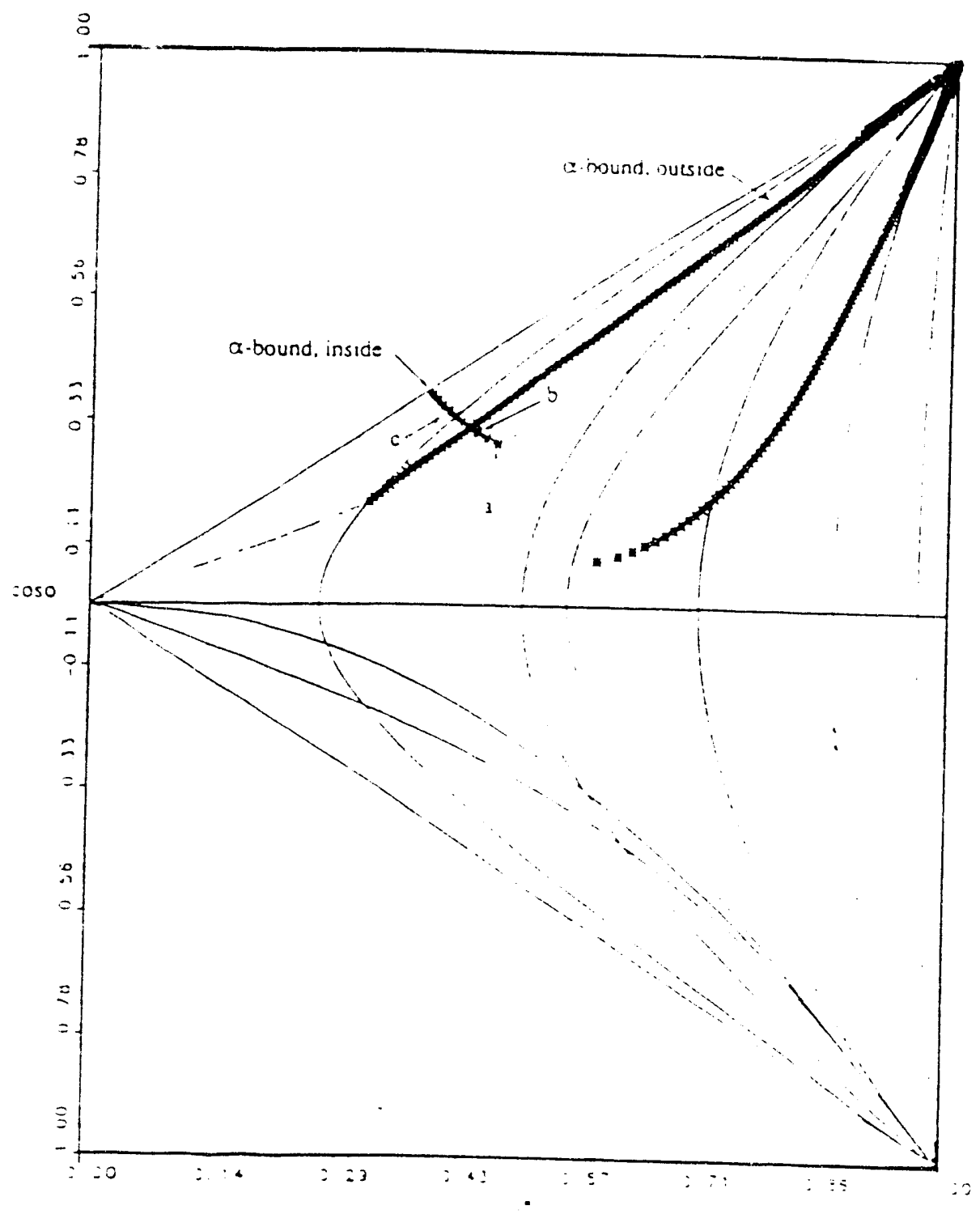

Figure 12. Phase space plot of the boundary points for the stable intertaces with contact angle $\theta=1)^{\circ}$. The soiid parameters are the same as in Figure 1. 


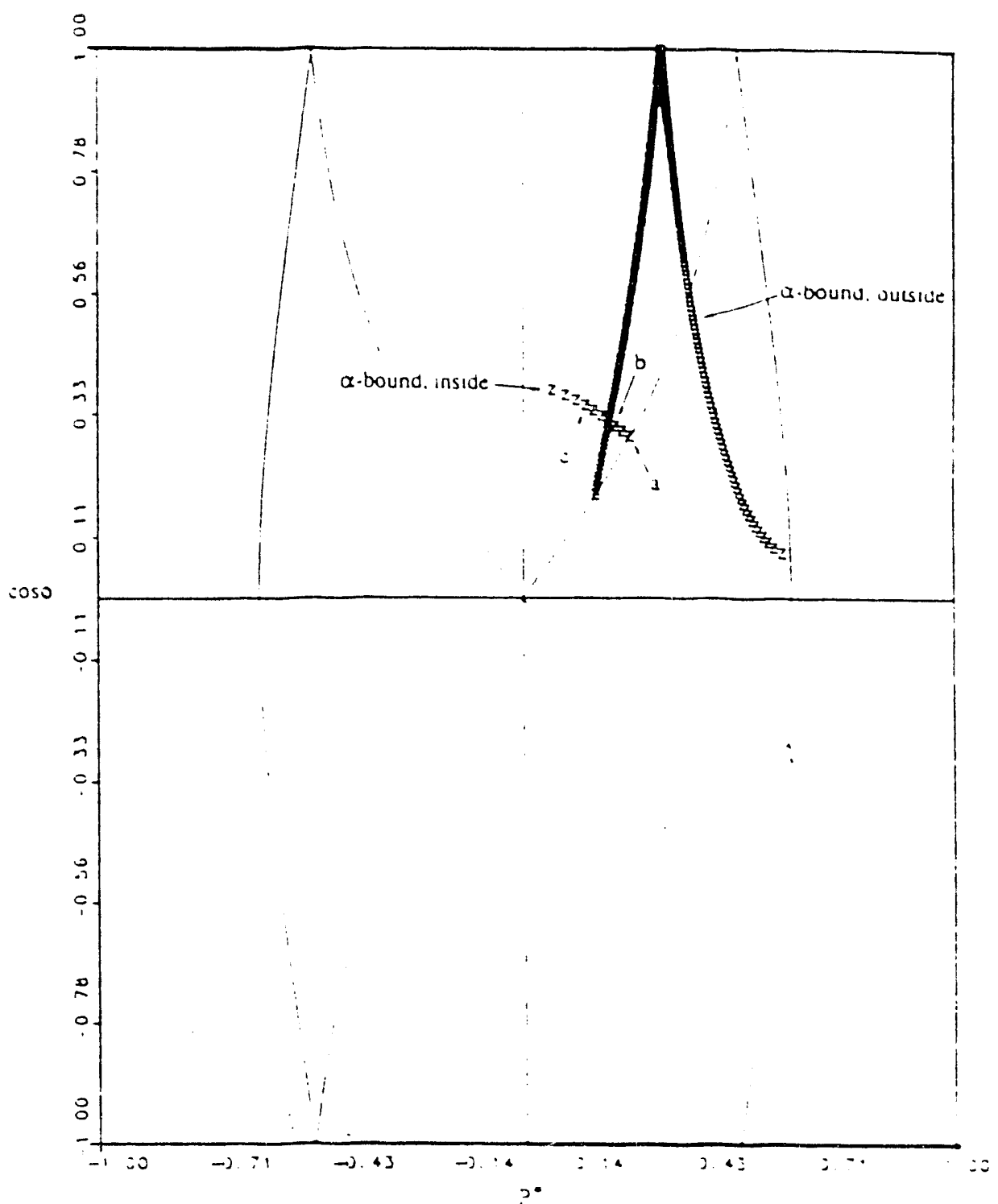

Figure 13. Arc length plot of the boundary points tor the stable intertaces with contact angie $\theta=0^{\circ}$. The solid zarameters are the same as in Figure 1. 

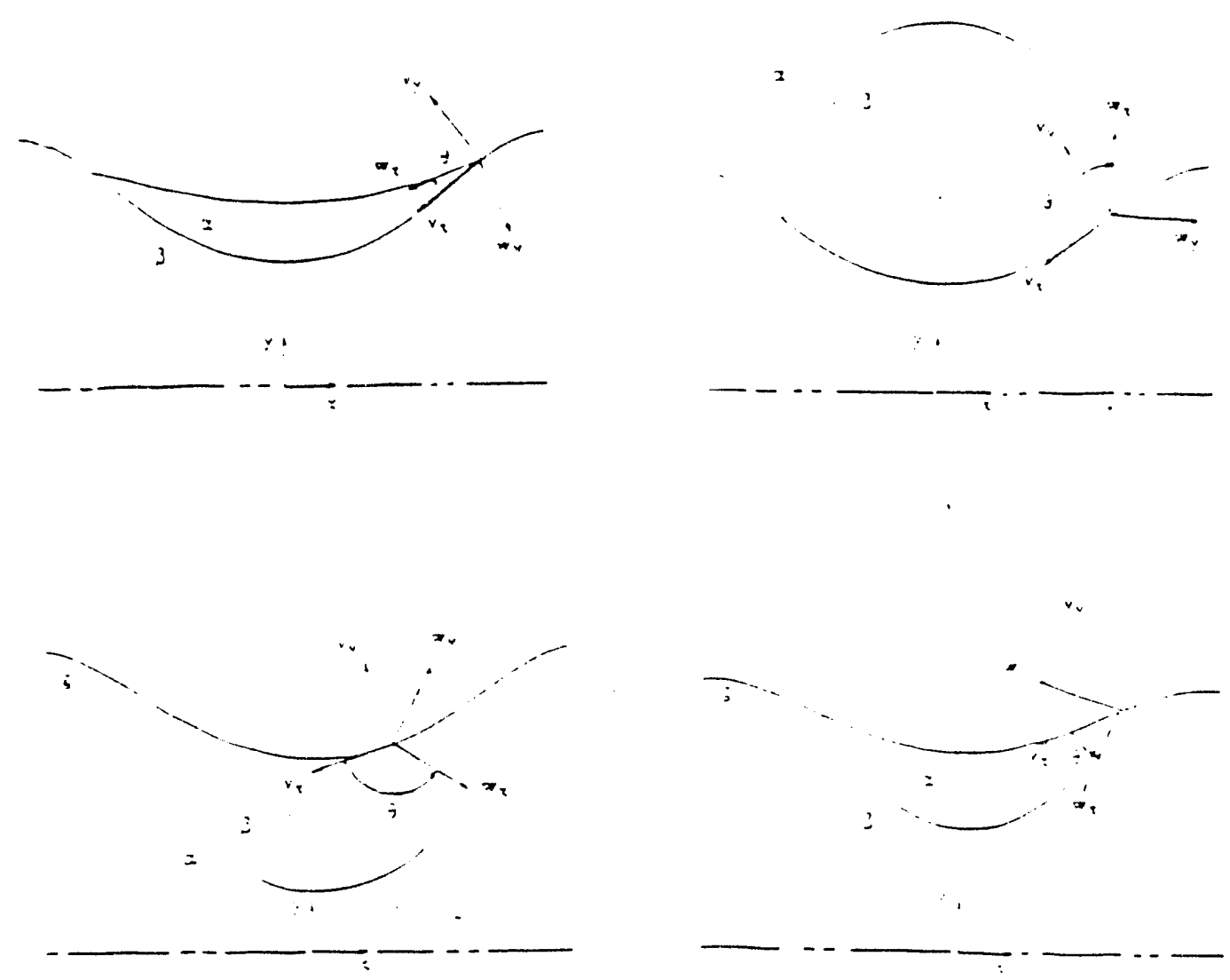

Figure 14. The four-phase contigurations showing the lncai coordinate systems. 


\section{Subtask 3c - Wettability and Adsorption}

\section{A New Model of Ionic Surfactant Adsorption at the Metal Oxide/Aqueous Solution Interface}

\section{Introduction}

The modeling of surfactant adsorption at the solid/solution interface has progressed from a simple Stern-Grahame approach, which is only successful in describing extremely low surface coverages, to complicated models which incorporate many of the important factors which influence surfactant adsorption. Thus far, however, no modei exists which links an adsorption isotherm with a quantitative description of the influence of the surfactant itself on the structure of the electrical double layer (EDL) at the solid/solution interface. The two simply cannot be treated as independent of one another, but until now, this has been the case.

The new model of surfactant adsorption presented here provides a means to calculate the influence of ionic surfactant adsorption on the EDL, and conversely, the influence of the EDL on the adsorption of the surfactant. The quantitative effects of such factors as as $\mathrm{pH}$, ionic strength, and temperature, surface chemistry of the substrate, and surfactant concentration and structure are all accounted for in this approach. While it is still far from being all-encompassing, this model promotes a clearer understanding of the complex relationship among the multitude of factors which ultimately dictate the adsorption behavior of surfactant under a given set of conditions at the metal oxide/aqueous solution interface.

\section{Review of the complexation site-binding model without surfactant}

The basis of the model of surfactant adsorption presented here is the complexation site-binding model (CSBM), which has been widely accepted as a suitable description of the electrical double layer at the metal oxide/aqueous solution interface. The CSBM treats amphoteric sites on the surface as if they were chemical species in solution, "reacting", or complexing, with potential-determining and indifferent electrolyte ions subject to intrinsic equilibrium constants (Yates, 1975; Davis et al., 1978).

These constants ar, lised in conjunction with the GCSG model of the EDL (Grahame, 1947) to describe the behavior of the interface in aqueous solution. The EDL is modeled as thres planes separated by layers of constant capacitance: (1) the surface (o) 
plane, where potential-determining ions are adsorbed; (2) the inner Helmholtz (b) plane (IHP) where complexed ions are adsorbed; and (3) the outer Helmholtz (d) plane (OHP) where the diffuse layer begins.

The intrinsic equilibrium constants are estimated from potentiometric titration data. The total number of ionizable sites is experimentally accessible through a variety of techniques and may also be estimated from the crystal lattice structure. The value of the outer layer capacitance is taken to be a constant whose value derives from studies on mercury and reversible electrodes. The inner layer capacitance is related to the dielectric constant of the inner layer as well as the distance of closest approach of the specifically adsorbed ions at the IHP. These values are not experimentally accessible, so this is an adjustable parameter in the model.

Knowledge of the equilibrium constants, the total number of ionizable sites, and the values of the integral capacitances of the two layers permits simultaneous solution of the model equation to yield values of charge densities and potentials at all three planes as a function of $\mathrm{pH}$ and electrolyte concentration. The distribution of various surface species is also calculated. The potential at the diffuse layer is assumed to be the same value measured in electrokinetic experiments. For a particular metal oxide/background electrolyte system, these parameters are taken to uniquely define the state of the EDL.

To account for the adsorption of surfactant, the CSBM has been extended. A description of the four modes of adsorption which occur according to the new model follows.

\section{Adsok tion Mode Ii complexation of a charged surfactant ion}

The first mode of adsorption proposed in the new model $:$, simply the complexation of a negatively charged surfactant ion with a positively charged site on the surface. This reaction is analogous to that of the complexation of an anion with a positive $\mathrm{MOH}_{2}^{+}$site (Yates, 1975; Davis et al., 1978).

$$
\begin{gathered}
\mathrm{K}_{\mathrm{S}}^{\mathrm{int}{ }^{*}} \\
\mathrm{MOH}_{2}^{+} \mathrm{S}^{-} \leftrightarrow \mathrm{MOH}^{*}+\mathrm{H}_{\mathrm{O}}^{+}+\mathrm{S}_{\beta}^{-}
\end{gathered}
$$




$$
\mathrm{K}_{\mathrm{S}}^{\mathrm{int}^{*}}=\frac{\left[\mathrm{MOH}^{*}\right]\left[\mathrm{H}_{\mathrm{O}}^{+}\right]\left[\mathrm{S}_{\beta}^{-}\right]}{\left[\mathrm{MOH}_{2}^{+} \mathrm{S}^{-}\right]}
$$

where $\left[\mathrm{MOH}_{2}^{+} \mathrm{S}^{-}\right]=$concentration of positively charged sites complexed with negative surfactant ions.

As in the case of anion complexation, the interaction energy comprises both an electrostatic contribution, which derives from the Coulombic force operating at the IHP on the charged head group, and a chemical contribution, which characterizes the affinity of the head group for the surface. Unlike the anion, however, the surfactant ion also possesses a distinctive characteristic: the hydrophobic moiety. The extra contribution made to the overall adsorption energy by the hydrophobic effect must also be accounted for.

First proposed by Cases et al. (1976) and later used by Scamehorn et al. (1982) is the concept of a coverage-dependent lateral interaction energy to account for the hydrophobic effect in surfactant adsorption. This idea will be adopted here. Lateral interactions between the hydrocarbon tails of the surfactant ions adsorbing at the IHP are incorporated in the expression for the equilibrium among the different species through an additional term which depends on the level of fractional surface coverage, designated by $q$. As surface coverage increases, this term increases in value and adsorption is favored ever. more.

It has been shown that the free energy of adsorption at the IHP of a surfactant monomer is given by (Harwell et al., 1985)

$$
\Delta G_{\mathrm{ads}}^{\mathrm{o}}=\mathrm{z}_{\mathrm{s}} \mathrm{e} \Psi_{\beta}+\mathrm{m}_{\mathrm{i}} \omega \theta
$$

where $\omega=$ lateral interaction energy per methyl group, $\mathrm{m}_{\mathrm{i}}=$ total number of methyl groups which are removed from the bulk soluiion to the IHP, and $\theta=$ fractional surface coverage and $\theta=\Gamma_{\mathrm{S}}^{\mathrm{q}} / \Gamma_{\text {monolayer }}$.

For the surfactant ion, the expression for its activity at the IHP differs from the expression for the activity of an anion at the IHP by an additional term:

$$
\left.\left[S_{\beta}^{-}\right]=S_{\text {bulk }}^{-}\right] \exp \left[\frac{\mathrm{e \Psi}_{\beta}}{\mathrm{kT}}\right] \exp \left[\frac{-\mathrm{m}_{\mathrm{i}} \omega \theta}{\mathrm{kT}}\right]
$$

The expression for the equilibrium constant is then given by 


$$
\mathrm{K}_{\mathrm{S}}^{\text {int }^{*}}=\frac{\left[\mathrm{MOH}^{*}\right]\left[\mathrm{H}_{\text {bulk }}^{+}\right]\left[\mathrm{S}_{\text {bulk }}^{-}\right] \exp \left[\frac{-\mathrm{e}\left(\Psi_{\mathrm{O}}-\Psi_{\beta}\right)}{\mathrm{kT}}\right]}{\left[\mathrm{MOH}_{2}^{+} \mathrm{S}^{-}\right]} \exp \left[\frac{-\mathrm{m}_{\mathrm{i}} \omega \theta}{\mathrm{kT}}\right]
$$

In the CSBM, the exact physical location of the sites is not specified. In the case of complexation of background electrolytes at the IHP, this poses no problem since adsorption levels never reach monolayer coverage. In the case of surfactant adsorption at the IHP, however, the physical dimension of the surfactant ion must be accounted for in the model; otherwise, a mathematical oversaturation of the surface would be possible. With a total number of 16 ionizable sites $/ \mathrm{nm}^{2}$ of surface area for $\mathrm{TiO}_{2}$, e.g., it is clear that at a monolayer coverage of 2.87 molecules $/ \mathrm{nm}^{2}$ (the value for the sulfonate head groups as calculated by Scamehorn et al., 1982), all sites cannot be considered "active" for reaction with surfactant. The concentration of sites available to the surfactant at any given level of adsorption is designated by $\left[\mathrm{MOH}^{*}\right]$ :

$$
\left[\mathrm{MOH}^{*}\right]=[\mathrm{MOH}][1-\theta]
$$

Clearly, as $\theta \rightarrow 1,\left[\mathrm{MOH}^{*}\right] \rightarrow 0$. This device is used to limit surface coverage at the IHP to physically realistic values. The approach resembles that of Keesom et al. (1988), who considered the adsorption of ionic surfactants onto hydrophobic membranes and that of Jung et al. (1988), who considered the adsorption of oleate onto geothite.

\section{Adsorption Mode II: adsorption of neutral surfactant_ion-counterion pairs}

The second mode visualized in the model is the adsorption of a neutral surfactant ion-counterion pair at the IHP. Since this neutral pair is not electrostatically attracted to the surface, adsorption due to this mechanism will only occur to any measurable extent if the environment at the surface is sufficiently favorable to stabilize its presence. In other words, some surfactant ions must already be residing at the IHP via complexation with positive sites before lateral interactions become great enough to stabilize a neutral pair at the IHP. In fact, a critical number $n$ of such groups must be present before neutral pair adsorption will occur. When the cluster forms, the neutral surfactant ion-counter:on pair occupies a corresponding pair of neutral $\mathrm{MOH}^{*}$ sites which are coordinated with the critical number of $\mathrm{MOH}_{2}^{+} \mathrm{S}^{-}$sites. 
The concept that even strong electrolyte may associate near a charged surface was recognized by investigators including Koopal (1986), Kallay et al. (1988), Jung et al. (1988), and indirectly by Harwell et al. (1985) who considered the possibility of counter-ion binding at the bottom of his proposed admicelle structure.

The reaction for the formation of the charged complex/neutral pair cluster is written

$$
\begin{gathered}
\mathrm{K}_{\mathrm{SC}}^{\mathrm{int}{ }^{*}} \\
\mathrm{MOH} \cdot \mathrm{S}^{-} \mathrm{MOH} \cdot \mathrm{C}^{+} \cdot \mathrm{nMOH}_{2}^{+} \mathrm{S}^{-} \leftrightarrow 2 \mathrm{MOH}^{*}+\mathrm{S}_{\beta}^{-}+\mathrm{C}_{\beta}^{+}+\mathrm{nMOF}_{2}^{+} \mathrm{S}^{-}
\end{gathered}
$$

The intrinsic equilibrium constant is given by

$$
\mathrm{K}_{\mathrm{SC}}^{\mathrm{int}^{*}}=\frac{\left[\mathrm{MOH}^{*}\right]^{2}\left[\mathrm{C}_{\beta}^{+}\right]\left[\mathrm{S}_{\beta}^{-}\right]\left[\mathrm{MOH}_{2}^{+} \mathrm{S}^{-}\right]^{\mathrm{n}}}{\left[\mathrm{MOH} \cdot \mathrm{S}^{-} \mathrm{MOH} \cdot \mathrm{C}^{+} \cdot \mathrm{nOH}_{2}^{+} \mathrm{S}^{-}\right]}
$$

where $\left[\mathrm{MOH} \cdot \mathrm{S}^{-} \mathrm{MOH} \cdot \mathrm{C}^{+} \cdot \mathrm{nMOH}_{2}^{+} \mathrm{S}^{-}\right]=$concentration of neutral $\mathrm{MOH}^{*}$ sites bound in the cluster. Substituting the appropriate expressions for ion activity at the IHP,

$$
\begin{aligned}
& \mathrm{K}_{\mathrm{SC}}^{\mathrm{int} t^{*}}=\frac{\left[\mathrm{MOH}^{*}\right]^{2}\left[\mathrm{C}_{\text {bulk }}^{+}\right] \exp \left[\frac{\mathrm{e \Psi}_{\beta}^{\prime}}{\mathrm{kT}}\right]\left[\mathrm{S}_{\text {bulk }}^{-}\right] \exp \left[\frac{\mathrm{e} \Psi_{\beta}}{\mathrm{kT}}\right]\left[\mathrm{MOH}_{2}^{+} \mathrm{S}^{-}\right]^{\mathrm{n}}}{\left[\mathrm{MOH} \cdot \mathrm{S}^{-} \mathrm{MOH} \cdot \mathrm{C}^{+} \cdot \mathrm{nMOH}_{2}^{+} \mathrm{S}^{-}\right]} \\
& \exp \left[\frac{-\mathrm{m}_{\mathrm{i}} \omega \theta \mathrm{k}}{\mathrm{kT}}\right] \\
& \mathrm{K}_{\mathrm{SC}}^{\mathrm{int}}=\frac{\left[\mathrm{MOH}^{*}\right]^{2}\left[\mathrm{C}_{\text {bulk }}^{+}\right]\left[\mathrm{S}_{\text {bulk }}^{-}\right]\left[\mathrm{MOH}_{2}^{+} \mathrm{S}^{-}\right]^{\mathrm{n}}}{\left[\mathrm{NOH}_{\mathrm{i}} \mathrm{S}^{-} \mathrm{MOH} \cdot \mathrm{C}^{+} \cdot \mathrm{nMOH}_{2}^{+} \mathrm{S}^{-}\right]} \exp \left[\frac{-\mathrm{m}_{\mathrm{i}} \omega \theta}{\mathrm{kT}}\right]
\end{aligned}
$$

Solving Eq. (2) for $\left[\mathrm{MOH}_{2}^{+} \mathrm{S}^{-}\right]$, 


$$
\left[\mathrm{MOH}_{2}^{+} \mathrm{S}^{-}\right]=\frac{\left[\mathrm{MOH}^{*}\right]\left[\mathrm{H}_{\text {bulk }}^{+}\right]\left[\mathrm{S}_{\text {bulk }}^{-}\right] \exp \left[\frac{-\mathrm{e}\left(\Psi_{\mathrm{O}}-\Psi_{3}\right)}{\mathrm{kT}}\right]}{\mathrm{K}_{\mathrm{S}}^{\mathrm{int}^{*}}} \exp \left[\frac{-\mathrm{m}_{\mathrm{i}} \omega \theta}{\mathrm{kT}}\right]
$$

Substituting this expression into Eq. (10) yields

$$
\begin{aligned}
& {\left[\mathrm{MOH} \cdot \mathrm{S}^{-} \mathrm{MOH} \cdot \mathrm{C}^{+} \cdot \mathrm{nMOH}_{2}^{+} \mathrm{S}^{-}\right]=\frac{\left[\mathrm{MOH}^{*}\right]^{\mathrm{n}+2}\left[\mathrm{C}_{\text {bulk }}^{+}\right]\left[\mathrm{H}_{\text {bulk }}^{+}\right]^{\mathrm{n}}\left[\mathrm{S}_{\text {bulk }}^{-}\right]^{\mathrm{n}+1}}{\mathrm{~K}_{\mathrm{SC}}^{\mathrm{int}}\left(\mathrm{K}_{\mathrm{S}}^{\mathrm{int}^{*}}\right)^{\mathrm{n}}}} \\
& \cdot \exp \left[\frac{-\mathrm{en}\left(\Psi_{\mathrm{O}}-\Psi_{\beta}\right)}{\mathrm{kT}}\right] \exp \left[\frac{-[\mathrm{n}+1] \mathrm{m}_{\mathrm{i}} \omega \theta}{\mathrm{kT}}\right]
\end{aligned}
$$

Since there are $(n+1)$ surfactant ions bound in each cluster and one surfactant ion bound in each complex, the total adsorption density due to both modes of IHP adsorption is given by

$$
\Gamma_{S}=\frac{\left[\mathrm{MOH}_{2}^{+} \mathrm{S}^{-}\right]+(\mathrm{n}+1)\left[\mathrm{MOH} \cdot \mathrm{S}^{-} \mathrm{MOH} \cdot \mathrm{C}^{+} \cdot \mathrm{nMOH}_{2}^{+} \mathrm{S}^{-}\right]}{\text {(surface area per unit volume) }}
$$

Figure 1 illustrates the two moces of surfactant adsorption included in the newly extended version of the CSBM.

\section{CSBM equations modified by Mode L and Mode IL adsorption}

Since negatively charged surfactant ions at the IHP will complex with positively charged sites on the surface, the expression for $\sigma_{O}$ and $\sigma_{\beta}$ must be modified:

$$
\begin{aligned}
& \sigma_{\mathrm{O}}=\frac{10^{2} \mathrm{~F}}{\mathrm{~A}}\left\{\left[\mathrm{MOH}_{2}^{+}\right]+\left[\mathrm{MOH}_{2}^{+} \mathrm{A}^{-}\right]+\left[\mathrm{MOH}_{2}^{+} \mathrm{S}^{-}\right]\right. \\
& \left.+\mathrm{n}\left[\mathrm{MOH} \cdot \mathrm{S}^{-} \mathrm{MOH} \cdot \mathrm{C}^{+} \cdot \mathrm{nMOH}_{2}^{+} \mathrm{S}^{-}\right]-\left[\mathrm{MO}^{-}\right]-\left[\mathrm{MO}^{-} \mathrm{C}^{+}\right]\right\} \\
& \sigma_{\beta}=\frac{10^{2} \mathrm{~F}}{\mathrm{~A}}\left\{\left[\mathrm{MO}^{-} \mathrm{C}^{+}\right]-\left[\mathrm{MOH}_{2}^{+} \mathrm{A}^{-}\right]-\left[\mathrm{MOH}_{2}^{+} \mathrm{S}^{-}\right]\right.
\end{aligned}
$$




$$
\text { - } \left.\mathrm{n}\left[\mathrm{MOH} \cdot \mathrm{S}^{-} \mathrm{MOH} \cdot \mathrm{C}^{+} \cdot \mathrm{nMOH}_{2}^{+} \mathrm{S}^{-}\right]\right\}
$$

The total site balance must also be rewritten to include the sites bound in the surfactant complexes and clusters:

$$
\begin{aligned}
& \mathrm{N}_{\mathrm{S}}=\frac{10^{-18} \mathrm{~N}_{\mathrm{A}}}{\mathrm{A}}\left\{[\mathrm{MOH}]+\left[\mathrm{MOH}_{2}^{+}\right]+\left[\mathrm{MO}^{-}\right]+\left[\mathrm{MOH}_{2}^{+} \mathrm{A}^{-}\right]\right. \\
& \left.+\left[\mathrm{MO}^{-} \mathrm{C}^{+}\right]+\left[\mathrm{MOH}_{2}^{+} \mathrm{S}^{-}\right]+(\mathrm{n}+2)\left[\mathrm{MOH} \cdot \mathrm{S}^{-} \mathrm{MOH} \cdot \mathrm{C}^{+} \cdot \mathrm{nMOH}_{2}^{+} \mathrm{S}^{-}\right]\right\}
\end{aligned}
$$

Mass balance equations are also required for all other species and must be revised to account for the presence of surfactant:

$$
\begin{aligned}
& {\left[\mathrm{C}_{\text {total }}^{+}\right]=\left[\mathrm{C}_{\text {bulk }}^{+}\right]+\left[\mathrm{MOC}^{+}\right]+\left[\mathrm{MOH} \cdot \mathrm{S}^{-} \mathrm{MOH} \cdot \mathrm{C}^{+} \cdot \mathrm{nMOH}_{2}^{+} \mathrm{S}^{-}\right]} \\
& {\left[\mathrm{A}_{\text {total }}^{-}\right]=\left[\mathrm{A}_{\text {bulk }}^{-}\right]+\left[\mathrm{MOH}_{2}^{+} \mathrm{A}\right]} \\
& {\left[\mathrm{S}_{\text {total }}^{-}\right]=\left[\mathrm{S}_{\text {bulk }}^{-}\right]+\left[\mathrm{MOH}_{2}^{+} \mathrm{S}^{-}\right]+(\mathrm{n}+1)\left[\mathrm{MOH} \cdot \mathrm{S}^{-} \mathrm{MOH} \cdot \mathrm{C}^{+} \cdot \mathrm{nOOH}_{2}^{+} \mathrm{S}^{-}\right]} \\
& {\left[\mathrm{C}_{\text {total }}^{+}\right]=\left[\mathrm{A}_{\text {total }}^{-}\right]+\left[\mathrm{S}_{\text {total }}^{-}\right]}
\end{aligned}
$$

The Gouy-Chapman equation relating diffuse layer potential to charge is also influenced by the presence of surfactant through the ionic strength:

$$
\sigma_{\mathrm{d}}=-\sqrt{8 k T \varepsilon_{\mathrm{O}} \varepsilon \mathrm{N}_{\mathrm{A}} \mathrm{I}} \sinh \left[\frac{\mathrm{e} \Psi_{\mathrm{d}}}{2 k \mathrm{~T}}\right]
$$

where

$$
\mathrm{I}=0.5\left\{\left[\mathrm{C}_{\text {bulk }}^{+}\right]+\left[\mathrm{A}_{\text {bulk }}^{-}\right]+\left[\mathrm{H}_{\text {bulk }}^{+}\right]+\left[\mathrm{OH}_{\text {bulk }}^{-}\right]+\left[\mathrm{S}_{\text {bulk }}^{-}\right]\right\}
$$

The GCSG model equations are 


$$
\begin{aligned}
& \sigma_{\mathrm{O}}=\mathrm{C}_{1}\left(\Psi_{\mathrm{O}}-\Psi_{\beta}\right) \\
& \sigma_{\mathrm{d}}=-\mathrm{C}_{2}\left(\Psi_{\beta}-\Psi_{\mathrm{d}}\right) \\
& \sigma_{\mathrm{O}}+\sigma_{\beta}=-\sigma_{\mathrm{d}}
\end{aligned}
$$

The expressions for the concentrations of the other surface species also remain unchanged and are also repeated here for the sake of completeness:

$$
\begin{aligned}
& {\left[\mathrm{MOH}_{2}^{+}\right]=\frac{[\mathrm{MOH}]\left[\mathrm{H}_{\text {bulk }}^{+}\right] \exp \left[\frac{-\mathrm{e} \Psi_{\mathrm{O}}}{\mathrm{kT}}\right]}{\mathrm{K}_{\mathrm{a}_{1}}^{\text {int }^{*}}}} \\
& {\left[\mathrm{MO}^{-}\right]=\frac{\mathrm{K}_{\mathrm{a}_{2}}^{\mathrm{int}^{*}}[\mathrm{MOH}] \exp \left[\frac{\mathrm{e} \Psi_{\mathrm{O}}}{\mathrm{kT}}\right]}{\left[\mathrm{H}_{\text {bulk }}^{+}\right]}} \\
& {\left[\mathrm{MOH}_{2}^{+} \mathrm{A}^{-}\right]=\frac{[\mathrm{MOH}]\left[\mathrm{H}_{\text {bulk }}^{+}\right]\left[\mathrm{A}_{\text {bulk }}^{-}\right] \exp \left[\frac{-\mathrm{e}\left(\Psi_{\mathrm{O}}-\Psi_{\beta}\right)}{\mathrm{kT}}\right]}{\mathrm{K}_{\mathrm{A}}^{\mathrm{int}}}} \\
& {\left[\mathrm{MO}^{-}\right]=\frac{\mathrm{K}_{\mathrm{C}}^{\mathrm{int}}\left[\mathrm{C}_{\text {bulk }}^{+}\right] \exp \left[\frac{\mathrm{e}\left(\Psi_{\mathrm{O}}-\Psi_{\beta}\right)}{\mathrm{kT}}\right]}{[\mathrm{MOH}]\left[\mathrm{H}_{\text {bulk }}^{+}\right]}}
\end{aligned}
$$

The extended form of the CSBM to include surfactant adsorption at the IHP consists of the following seventeen independent equations: the concentrations of the surface species, Eqs. (11), (12), and (26) to (29); the charge density, Eqs. (14) and (15); the mass balance equations on surface species and solution species, Eqs. (16) to (20); and the GCSG model, Eqs. (21) and (23) to (25). In addition to the original CSBM parameters $\mathrm{N}_{\mathrm{S}}, \mathrm{K}_{\mathrm{a}_{1},}^{\mathrm{int}}, \mathrm{K}_{\mathrm{a}_{2}}^{\mathrm{int}}, \mathrm{K}_{\mathrm{C}}^{\mathrm{int}}, \mathrm{K}_{\mathrm{A}}^{\mathrm{int}}, \mathrm{C}_{1}$, and $\mathrm{C}_{2}$, the following parameters have been introduced: $\mathrm{K}_{\mathrm{S}}^{\mathrm{int}}, \mathrm{K}_{\mathrm{SC}} \mathrm{int}^{*}, \mathrm{~m}_{\mathrm{i}}, \omega$, and $\mathrm{n}$. 
The special nature of the surfactant, namely the hydrophobic effect, is accounted for through these two modes of adsorption in several ways. In the formation of the $\mathrm{MOH}_{2}^{+} \mathrm{S}$ complex, interaction of the hydrocarbons tails with the surface is accounted for through the value of the equilibrium constant $\mathrm{K}_{\mathrm{S}}^{\mathrm{int}}{ }^{*}$, which is less than intrinsic equilibrium constants for anions without hydrophobic moiety. The lower value of the complexation constant means that surfactant anions are preferentially complexed at positive sites over other anions present in solution. In both complex formation and cluster formation, tail-tail interaction is accounted for through the additional free energy term $\exp \left[\mathrm{m}_{\mathrm{i}} \omega \theta / \mathrm{kT}\right]$, which is a function of surface coverage, $\theta$, and lateral interaction energy, $\omega$, of the tails. As surface coverage increases, the value of the term increases; and since it multiplies the complex concentration term, the net effect is the enhancement of complexation (or, equivalently, adsorption) with increasing surface coverage. In the cluster formation reaction, tail-tail interaction is also accounted for through the dependence of neutral-site adsorption on positive-site adsorption. As the surface environment becomes more hydrophobic with increasing complexation of surfactant ions with positive sites, in-filling by neutral surfactant-counterion pairs is favored.

\section{Other contributions to adsorption}

Adsorption Mode III: Second-Layer Formation

If adsorption due to Modes I and II is complete, the surface is completely saturated at the IHP by a complete monolayer of surfactant. In many cases, however, adsorption is observed to exceed monolayer coverage; and, in fact, may reach saturation at complete bilayer coverage. To account for this possibility, the third mode of adsorption envisioned in this model is the formation of a second layer on top of the first layer which is forming at the IHP.

Following the approach of Scamehorn et al. (1982), the second-layer isotherm takes the form of a modified Fowler-Guggenheim isotherm with lateral interactions in the second layer:

$$
\frac{\theta_{2}}{\theta_{1}-\theta_{2}}=\mathrm{K}_{2} \mathrm{f}_{\mathrm{S}}\left[\mathrm{S}_{\text {buik }}^{-}\right] \exp \left[\frac{-\omega \mathrm{m}_{\mathrm{T}} \theta_{2}}{\mathrm{kT}}\right]
$$

where $\theta_{1}=$ first-layer fractional surface coverage at the IHP due to adsorption via modes I and II, $\theta_{2}=$ second-layer fractional coverage, $\omega=$ lateral interaction energy per methyl 
group, $\mathrm{m}_{\mathrm{T}}=$ total number of methyl groups in tail, $\mathrm{K}_{2}=$ equilibriun constant for second-layer adsorption, and $\mathrm{f}_{\mathrm{S}}=$ surfactant activity coefficient.

$\mathrm{K}_{2}$ is a constant which defines the reference state of the system and is a function of $\omega$. The surfactant activity coefficient, $f_{S}$, is a strong function of background electrolyte concentration. The number of interacting methyl groups is taken to be the total number of groups in the tail.

For very low levels of first-layer coverage, this term will be negligible; but as first layer coverage increases, second-layer coverage may become significant. Its incorporation in the model is therefore essential.

\section{Adsorption Mode IV: Diffuse Layer Adsorption}

The final mode of adsorption visualized in the new model is that which arises due to the potential at the OHP where the diffuse layer begins. Van den Hul and Lyklema (1967) recognized this effect and successfully used it to calculate surface areas of charged particles dispersed in solution. Since the interface at the OHP is charged, counterions will show a preference for the interface and accumulate there. Coions will be expelled. If the potential at the OHP is positive, positive adsorption of surfactant from the bulk solution will occur due to favorable electrostatic interactions. If the potential at the OHP is negative, negative adsorption will occur and the bulk solution will actually be enriched in surfactant as unfavorable electrostatic interactions repel surfactant ions from the OHP.

The surface deficit (or accumulation) due to electrostatic interaction with the OHP is given by

$$
\Gamma_{S}^{O H P}=\int_{d}^{\infty}\left\{\left[S_{\text {bulk }}^{-}\right]-\left[S^{-}(x)\right]\right\} d x
$$

The total ion exclusion (attraction) from (to) a surface of surface area, SA, is SA $-\Gamma_{S}^{O H P}$ A material balance may be used to relate this to the increase in bulk concentration, $\Delta\left[S_{\text {bulk }}^{-}\right]$. Consider a dispersion with a surface area of $\mathrm{SA} \mathrm{cm}$ in a total solution volume of $\mathrm{V}_{\mathrm{t}} \mathrm{cm}^{3}$ at an initial concentration, $\left[\mathrm{S}_{\text {total }}^{-}\right]$. Some ions will complex at the IHP, resulting in a solution concentration of $\left[\mathrm{S}_{\text {total }}^{-}\right]-\left[\mathrm{S}_{\mathrm{IHP}}^{-}=\left[\mathrm{S}_{\text {bulk }}^{-*}\right]\right.$. If the charge on the OHP is negative, it repels surfactant ions so that their bulk concentration rises to $\left[\mathrm{S}_{\text {total }}^{-}\right.$] except in a small volume from which $S \cdot \Gamma_{S}^{O H P}$ ions are excluded. The total amount of surfactant ions is $V\left[S_{\text {bulk }}^{-}\right]_{--}^{-}$ $\mathrm{SA} \cdot \Gamma_{\mathrm{S}}^{\mathrm{OHP}}$. From a material balance, 


$$
\begin{aligned}
\mathrm{V}_{\mathrm{t}}\left[\mathrm{S}_{\text {bulk }}^{-*}\right] & =\mathrm{V}_{\mathrm{t}}\left[\mathrm{S}_{\text {bulk }}^{-}\right]-\mathrm{SA} \cdot \Gamma_{\mathrm{S}}^{\mathrm{OHP}} \\
\mathrm{SA} \cdot \Gamma_{\mathrm{S}}^{\mathrm{OHP}} & =\mathrm{V}_{\mathrm{t}}\left[\mathrm{S}_{\text {bulk }}^{-}\right]-\left[\mathrm{S}_{\text {bulk }}^{-*}\right] \\
\mathrm{SA} \cdot \Gamma_{\mathrm{S}}^{\mathrm{OHP}} & =\mathrm{V}_{\mathrm{t}} \Delta\left[\mathrm{S}_{\text {bulk }}^{-}\right]
\end{aligned}
$$

Therefore,

$$
\Gamma_{\mathrm{S}}^{\mathrm{OHP}}=\frac{\mathrm{V}_{\mathrm{t}}}{\mathrm{SA}} \Delta\left[\mathrm{S}_{\text {bulk }}^{-}\right]
$$

According to the Boltzmann principle,

$$
\left[S^{-}(x)\right]=\left[S_{\text {bulk }}^{-}\right] \exp \left[\frac{\mathrm{e}^{\Psi}(\mathrm{x})}{\mathrm{kT}}\right]
$$

Therefore,

$$
\Gamma_{S}^{\mathrm{OHP}}=\left[\mathrm{S}_{\text {bulk }}^{-}\right] \int_{\mathrm{d}}^{\infty}\left\{1-\exp \left[\frac{\mathrm{e} \Psi(\mathrm{x})}{\mathrm{kT}}\right]\right\} \mathrm{dx}
$$

Combining Eqs. (35) and (37),

$$
\Delta\left[\mathrm{S}_{\text {bulk }}^{-}\right]=\frac{\mathrm{SA}\left[\mathrm{S}_{\text {bulk }}^{-}\right]}{\mathrm{V}_{\mathrm{t}}} \int_{\mathrm{d}}^{\infty}\left\{1-\exp \left[\frac{\mathrm{e} \Psi(\mathrm{x})}{\mathrm{kT}}\right]\right\} \mathrm{dx}
$$

Using this equation, together with the Poisson-Boltzmann equation for a symmetrical 1:1 electrolyte, the adsorption due to electrostatic interaction with the OHP may be shown to be:

$$
\Gamma_{S}^{\mathrm{OHP}}=\mathrm{N}_{\mathrm{A}} \cdot 10^{6.5} \frac{2 \mathrm{kT}}{\mathrm{e}} \frac{\sqrt{\left[\mathrm{S}_{\text {bulk }}^{-}\right]}}{\sqrt{\frac{2 \mathrm{~N}_{\mathrm{A}} \mathrm{kT}}{\varepsilon \varepsilon_{\mathrm{o}}}}}\left\{1-\exp \left[\frac{\mathrm{e} \Psi \mathrm{d}}{2 \mathrm{kT}}\right]\right\}
$$


where $\Gamma_{S}^{O H P}$ is in units of molec/m², and $\left[S_{\text {bulk }}^{-}\right]$is in units of moles/liter.

Since the calculation of OHP adsorption relies strictly on Coulombic interactions due to the charge on the ion, any differences in structure are irrelevant. In the presence of a background 1:1 electrolyte, the OHP adsorption of surfactant is calculated by replacing $[S \backslash S(-$, bulk $)]$ in Eq. (39) with $\{[A \backslash S(-$, bulk $)]+[S \backslash S(-$, bulk $)]\}$, then multiplying $\cap S(O H P, S)$

by the expression $\left[S_{\text {bulk }}^{-}\right] /\left\{\left[A_{\text {bulk }}^{-}\right]+\left[S_{\text {bulk }}^{-}\right]\right\}$to determine the fraction of negative ion adsorption attributable to surfactant. The contribution of $\left(\mathrm{OH}^{-}\right)$is neglected since it is always several orders of magnitude lower than that of other anions present.

\section{The comprehensive model of surfactant adsorption}

Revision of the Electrical Double Layer to Reflect Surfactant Adsorption

With the adsorption of a second layer of surfactant on top of the first layer adsorbed at the IHP due to Modes I and II, the structure of the electrical double layer will change. As the second layer forms, an adjunct plane, designated as the IHP*, is visualized as cutting through the centers of the surfactant head groups and bound counterions. Associated with this adjunct compact layer is a charge density, $\sigma_{\beta}$, and a potential, $\Psi_{\beta}^{*}$. Now, $\mathrm{C}_{2}$ is the integral capacitance of the layer separating the IHP and the IHP*; the integral capacitance separating the extended $\mathrm{IHP}^{*}$ from the beginning of the diffuse layer at the OHP is designated by $\mathrm{C}_{2}$. The GCSG equations must be revised to describe the double layer after the adsorption of a second layer of surfactant.

As betore, the potential drop between the surface and the IHP is given by

$$
\sigma_{0}=C_{1}\left(\Psi_{0}-\Psi_{\beta}\right)
$$

The potential drop between the IHP and the IHP* is given by

$$
\sigma_{O}+\sigma_{\beta}=C_{2}\left(\Psi_{\beta}-\Psi_{\beta}^{*}\right)
$$

The charge density at the IHP* is related to the concentration of surfactant adsorbed in the second layer and the concentration of any bound counterions $\mathrm{C}_{\mathrm{ci}}$ by

$$
\sigma_{\beta}^{*}=\frac{10^{2} \mathrm{~F}}{\mathrm{~A}} \mathrm{C}_{\mathrm{ci}}-\frac{10^{20} \mathrm{~F}}{\mathrm{~N}_{\mathrm{A}}}\left[\theta_{2} \cdot \Gamma_{\text {monolayer }}\right]
$$


The potential drop between the IHP* and the OHP is given by

$$
\sigma_{O}+\sigma_{\beta}+\sigma_{\beta}^{*}=C_{2}^{*}\left(\Psi_{\beta}^{*}-\Psi_{d}\right)
$$

The electroneutrality condition is now

$$
\sigma_{\mathrm{O}}+\sigma_{\beta}+\sigma_{\beta}=-\sigma_{\mathrm{d}}
$$

With this relationship, Eq. (32) may be rewritten in a form analogous to that of the original GCSG equations:

$$
-\sigma_{d}=\cdot C_{2}^{*}\left(\Psi_{\beta}^{*}-\Psi_{d}\right)
$$

Figure 2 schematically depicts the four modes of ionic surfactant adsorption at the metal oxide/aqueous solution interface.

Some Simplifications and Assunptions in the Teatment of the Model

To make the solution of the model tractable, some simplifications and assumptions are necessary. The major ones are discussed here.

Since second-layer coverage is not expected to be significant until first-layer coverage is substantial, its influence on the overall structure of the double layer will be neglected. In this case, the IHP* disappears, so the equations describing the EDL revert to the original GCSG form.

The values of the integral capacitances, $C_{1}$ and $C_{2}$, are expected to change with increasing coverage. As the surface becomes more saturated with surfactant, the dielectric constant of of the region between the IHP and the OHP should decrease. As packing density of the head groups increases, the dielectric constant of the region between the surface and the IHP should change as well. While possible changes in the values of $C_{1}$ and $\mathrm{C}_{2}$ with changes in solution composition were recognized by the developers of the CSBM, the effect was neglected in the original formulation of the model, and it is neglected in the extended model presented here. 
The authors of the CSBM also recognized the discreteness-of-charge effect but chose to neglect it as well (Yates, 1975; Davis et al., 1976). In keeping with their approach, the effect is not considered in this model.

Surface heterogeneity is also not considered in this model. Because the CSBM is based on the assumption of smeared-out potentials and average values of charge density at the various planes in the EDL, it does not lend itself well to modeling of heterogeneity without a priori assumptions regarding the distribution of energies and/or locations of the various types of sites. Until experimental techniques are developed which can better elucidate the structure of the EDL on a microscopic scale, it seems unwarranted to complicate the model with such assumptions.

\section{Calculation of Surfactant Adsorption Density}

The new model of surfactant adsorption is mathematically expressed through the seventeen nonlinear equations of the extended ESBM, together with Eq. (30), which describes second-layer formation, and Eq. (39), which describes adsorption due to electrostatic interaction with the OHF. To calculate the adsorption density for a particular solution composition, the equations of the CSBM are simultaneously solved for the unknown quantities in the model: the potentials and charge densities at the three planes of the EDL, the distribution of the various types of ions through the EDL, and the distribution of the various types of species on the surface. At this point, adsorption density due to Modes I and II may be calculated:

$$
\Gamma_{\mathrm{S}}^{\mathrm{I}+\mathrm{II}}=\frac{\left[\mathrm{MOH}_{2}^{+} \mathrm{S}^{-}\right]+(\mathrm{n}+1)\left[\mathrm{MOH}^{*} \cdot \mathrm{S}^{-} \mathrm{MOH}^{*} \cdot \mathrm{C}^{+} \cdot \mathrm{nMOH}_{2}^{+} \mathrm{S}^{-}\right]}{(\text {surface area per unit volume })}
$$

Given $\Gamma_{\mathrm{S}}^{\mathrm{I}}+\Pi$, second layer adsorption density due to Mode III may be calculated by solving the following equation:

$$
\frac{\Gamma_{\mathrm{S}}^{\mathrm{III}}}{\Gamma_{\mathrm{S}}^{\mathrm{III}}-\Gamma_{\mathrm{S}}^{\mathrm{III}}}=\mathrm{K}_{2} \mathrm{f}_{\mathrm{S}}\left[\mathrm{S}_{\text {bulk }}^{-}\right] \exp \left[\frac{-\omega \mathrm{m}_{\mathrm{T}} \theta_{2}}{\mathrm{kT}}\right]
$$

where $\theta_{2}=$ second-layer fraci ional coverage $\Gamma_{S}^{I I I} / \Gamma_{\text {Smonolayer }}$.

Since the effect of sec ind-layer coverage on the overall structure of the EDL has been neglected, adsorption density w'e to Mode IV may be calculated: 


$$
\begin{aligned}
& \mathrm{i}_{\mathrm{S}}^{\mathrm{IV}}=\mathrm{N}_{\mathrm{A}} \cdot 10^{6.5 \frac{2 \mathrm{kT}}{\mathrm{e}}} \frac{\left[\mathrm{S}_{\text {bulk }}^{-}\right]}{\left\{\left[\mathrm{A}_{\text {bulk }}^{-}\right]+\left[\mathrm{S}_{\text {bulk }}^{-}\right]\right\}} \\
& \mathrm{x} \\
& \sqrt{\frac{\sqrt{\frac{2 \mathrm{~N}_{\mathrm{A}} \mathrm{kT}}{\varepsilon_{\mathrm{o}}}}}{\left.\left.\mathrm{A}_{\mathrm{bulk}}^{-}\right]+\left[\mathrm{S}_{\text {bulk }}^{-}\right]\right\}}}\left\{1-\exp \left[\frac{\mathrm{e} \Psi \mathrm{d}}{2 \mathrm{kT}}\right]\right\}
\end{aligned}
$$

The effect of adsorption due to 'Modes III and IV on the on the value of $S_{\text {total' }}^{-}$ which is an unknown quantity in the extended CSBM, has been neglected. This simplification, together with the assumption that the IHP* may be neglected, act to decouple the extended CSBM equations from Eqs. (47) and (48); this is necessary to make the system of equations computationally tractable.

The total adsorption density at a given solution composition may now be calculated from Eqs. (46 to (48).

\section{Parameter Determination}

In the extension of the original CSBM to include surfactant adsorption due to Modes I and II at the IHP, the following parameters have been introduced into the model: the intrinsic surfactant complexation equilibrium constant, $\mathrm{K}_{\mathrm{S}}^{\text {int }}{ }^{*}$; the intrinsic surfactant clustering equilibrium constant, $\mathrm{K}_{\mathrm{SC}}^{\text {int; }}$; the number of interacting methyl groups, $\mathrm{m}_{\mathrm{i}}$; the lateral interaction energy, $\omega$; and the coordination number of complexed groups per cluster, $\mathrm{n}$. One additional parameter is the equilibrium constant, $\mathrm{K}_{2}$, relating Mode III adsorption due to second-layer formation to adsorption due to Modes I and II.

A critical assumption made in extending the CSBM to include surfactant adsorption is that the parameters which describe the metal oxide/aqueous solution interface as determined in the absence of surfactant do not change when surfactant is present. $\mathrm{N}_{\mathrm{S}}$ is calculated from geometrical considerations of surface structure and confirmed experimentally, using a variety of complementary techniques. Its value is completely independent of the solution composition. $\mathrm{C}_{2}$ is assigned the same value for all metal oxides. The values of $\mathrm{K}_{\mathrm{a}_{1}}^{\mathrm{int}}, \mathrm{K}_{\mathrm{a}_{2}}^{\mathrm{int}}, \mathrm{K}_{\mathrm{C}}^{\mathrm{int}}$, and $\mathrm{K}_{\mathrm{A}}^{\mathrm{int}{ }^{*}}$ are estimated from experimentally derived values of surface charge density, as measured in the absence of surfactant. These parameters, together with $C_{1}$, are optimized to generate the best fit of the surface charge 
density and complementary zeta-potential measurements, also taken in the absence of surfactant, over the wikest possible range of solution composition. Since they are explicitly independent of surfactant concentration, the values of the intrinsic equilibrium constants should not change in the presence of surfactant.

To describe surfactani adsorption, the parameters which neej to be determined, then, are $\mathrm{K}_{\mathrm{S}}^{\mathrm{int}}{ }^{*}, \mathrm{~K}_{\mathrm{SC}}^{\mathrm{int}}, \mathrm{m}_{\mathrm{i}}, \omega, \mathrm{n}$, and $\mathrm{K}_{2}$. The parameters should have physically reasonable values which change in a logical way with changes in system properties. A stringent test of the parameter values is that they successfully predict all the observed trends in experimentally ferived values of adsorption density, surface charge density, and zeta potential over a wide range of $\mathrm{pH}$, equilibrium surfactant concentration, background electrolyte concentration, and temperature.

Since the effect of second-layer coverage on the EDL has been neglected, it has been added in an ad hoc sense. The model is therefore expected to predict low levels of adsorption density well, due to the detailed consideration of the EDL, and to describe high levels of adsorption density only to a limited extent.

Since second-layet adsorption is assumed to be negligible until high levels of

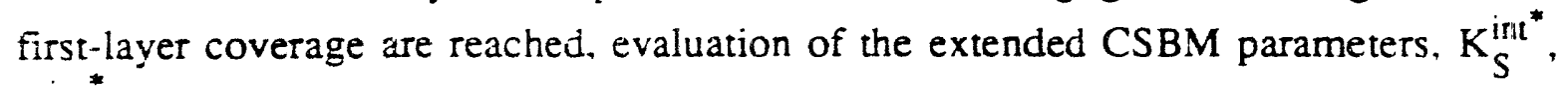
$\mathrm{K}_{\mathrm{SC}}^{\mathrm{int}}, \mathrm{m}_{\mathrm{i}}, \omega$, and $\mathrm{n}$, is based on regions in the adsorption surfaces where surface coverage is less than one monolayer thick. This condition holds true except for the very lowest $\mathrm{pH}$ values and highest bulk sufactant concentration values.

Methods of estimation and expected trends with changes in system properties are now outlined for each parameter.

\section{Lateral Interaction Parameter}

Estirizie of value. Based on conclusions drawn from studies of micellization regarding free energy of formation of micelles from monomers in solution, the value used for $\omega$ is $-0.59 \mathrm{kcal} / \mathrm{mivi}$, or $\sim-1 \mathrm{kT}$, per interacting methyl group for solutions at 30 to $40^{\circ} \mathrm{C}$. Cases et al. (1975) determined values for $\omega$ rangin from $^{-0.8}$ to $-1.14 \mathrm{kT}$ per methyl group for the adsorption of n-alkylamine chlc des on calcite.

Temperature effect. Since $\omega$ is expressed in terms of temperature its value will automatically reflect temperarure changes.

Structure effect. Since $\omega$ is expressed per unit methyl group, its value will automatically reflect changes in structure.

pH and salinity effect. Studies of micellization have demonstrated that the value of $\omega$ is neariy independent of $\mathrm{pH}$ and salinity (Scamehom et al. 1982). 


\section{Number of Interacting Groups}

Estimate of value. For surfactants adsorbing at the IHP, the number of methyl groups which actually interact with $\mathrm{m}_{\mathrm{i}}$ is expected to be less than the total number of methyl groups, $\mathrm{m}_{\mathrm{T}}$, in the tail. Since the molecules are not densely packed on the surface until coverage is very high, the tail groups nearest the head group may not be able to interact. As coverage increases, $m_{i}$ might increase as the molecules reside closer together. In the simplest case, however, $\mathrm{m}_{\mathrm{i}}$ has been treated as a constant subject to the condition that $\mathrm{m}_{\mathrm{i}}<\mathrm{m}_{\mathrm{T}}$

Temperature effect. The influence of temperature on the hydrophobic effect is complex (Bourrel and Schechter, 1988), so its influence on $\mathrm{m}_{\mathrm{i}}$ is not intuitively clear. The value of $\mathrm{m}_{\mathrm{i}}$ is not expected to be influenced by temperature over the narrow range considered in this study; but changes in $\mathrm{m}_{\mathrm{i}}$ in the case of wide variations in temperature cannot be ruled out.

Structure effect. The value of $\mathrm{m}_{\mathrm{i}}$ is expected to change in a systematic fashion with structure, as might be expected by analogy with Traube's rule: $\Delta \mathrm{m}_{\mathrm{i}}=\Delta \mathrm{m}_{\mathrm{T}}$. For a tail with 12 methyl groups, if $m_{i}$ is 6 , a tail with 10 methyl groups should have $m_{i}=4$.

$\mathrm{pH}$ and salinity effect. The number of interacting methyl groups is assumed to be independent of both $\mathrm{pH}$ and salinity.

Intrinsic Surfactant Compie, :ation Equilibrium Constant

Estimate of value. As has been noted, $\mathrm{pK}_{\mathrm{S}}^{\mathrm{ini}}$ is expected to have a higher value than $\mathrm{pK}_{\mathrm{A}}^{\text {int }^{*}}$ since the affinity of a surfactant ion for the surface should be greater than that of an anion with no hy'drophobic moiety. To estimate its value, data at very low equilibrium surfactant concentration at low background electrolyte is considered. In this range of solution composition, the effects of lateral interaction and neutral-pair adsorption are minimized, and monomers are adsorbed primarily due to the normal head-surface interaction which is characterized by $\mathrm{K}_{\mathrm{S}}^{\text {int }}{ }^{*}$.

Temperature effect. Since $\mathrm{K}_{\mathrm{S}}^{\text {Int }^{*}}$ primarily reflects the normal head-surface interaction, it is expected to increase with increasing temperature due to thermal fluctuations which tend to impede adsorption, at least over the narrow range of temperature studied here; but because the influence of temperature on the hydrophobic effect is complex, this trend may not be strictly followed over a wide range of temperatures.

Structure effect. $\mathrm{K}_{\mathrm{S}}^{\mathrm{int}^{*}}$ is expected to change to reflect the difference in hydrophobic effect caused by structural alteration of the surfactant. As the hydrophobic effect decreases, 
e.g., due to a decrease in tail length, the value of $\mathrm{pK}_{\mathrm{S}}^{\text {int }}{ }^{*}$ will decrease toward the value of $\mathrm{pK}_{\mathrm{A}}^{\mathrm{int}}{ }^{*}$ as the relative affinity of the surfactant for the surface decreases.

$\mathrm{pH}$ and salinity effect. Like the other intrinsic equilibrium constants in the CSBM, $\mathrm{K}_{\mathrm{S}}^{\mathrm{int}}{ }^{*}$ is derived so that it is independent of both $\mathrm{pH}$ and salinity.

\section{Intrinsic Surfactant Clustering Equilibrium Constant}

Estimate of value. Because a cluster contains several surfactant ions, it should have an overall affinity for the surface which is extremely high. To reflect this increase in interaction energy, the value of $\mathrm{K}_{\mathrm{SC}}^{\mathrm{int}}{ }^{*}$ is expected to be much lower than the value of $\mathrm{K}_{\mathrm{S}}^{\mathrm{int}}$, or equivalently $\mathrm{pK}_{\mathrm{SC}}^{\mathrm{int}}$ is much greater than $\mathrm{pK}_{\mathrm{S}} \mathrm{int}^{*}$.

Temperature effect. Over the narrow range of temperature studied here, $K_{S C}^{\text {int }^{*}}$ is expected to increase with increasing temperature to reflect increased thermal fluctuations which tend to reduce adsorption. Since the influence of temperature on the hydrophobic effect is complex; however, this may not be true over a wide range of temperatures.

Structure effect. Because the overall configuration of the cluster depends on the coordination number $n$, the effect of surfactant structure on $\mathrm{K}_{\mathrm{SC}}^{\mathrm{int}}{ }^{*}$ is not intuitively obvious, and will be discussed in terms of structural effects on $n$.

$\mathrm{pH}$ and salinity effect. As in the case of $\mathrm{K}_{\mathrm{S}}^{\mathrm{int}}, \mathrm{K}_{\mathrm{SC}}^{\mathrm{int}}$ is also derived so that it is independent of both $\mathrm{pH}$ and salinity.

Coordination Number of Complexed Sites in Cluster

Estimate of value. No clear intuitive picture is available to guide the choice of $n$, the number of $\mathrm{MOH}_{2}^{+} \mathrm{S}^{-}$groups required to stabilize the presence of a neutral surfactant ion-counterion pair at the IHP. In their studies of hemimicelle formation, Chander et al. (1983) proposed that for surfactant ions containing 10 to 14 carbon atoms, the structures which form at the onset of hemimicelle formation are dimers; this amounts to a coordination number of 1 . In their studies, they do not recognize the possibility of neutral-pair adsorption so the applicability of this conclusion is unclear, although it is probably a good estimate from which to start.

Temperature effect. The value of $n$ is expected to increase with temperature. As temperature increases, a greater number of $\mathrm{MOH}_{2}^{+} \mathrm{S}^{-}$groups is required to promote neutral-pair adsorption.

Structure effect. The value of $n$ will change to reflect changes in surfactant structure. With decreasing hydrophobic effect, n shor.ld increase; more $\mathrm{MOH}_{2}^{+} \mathrm{S}^{-}$groups are needed to stabilize the formation of a cluster. The value of $\mathrm{K}_{\mathrm{SC}}^{\mathrm{int}^{*}}$ wili decrease to reflect the greater overall affinity for the surface of a cluster which contains more surfactant ions. 
$\mathrm{pH}$ and salinity effect: The value of $\mathrm{n}$ is assumed to be independent of $\mathrm{pH}$ and salinity.

\section{Second-Layer Formation Equilibrium Constant}

Estimate of value. Second-layer adsorption should only become significant at high levels of first-layer adsorption. The value of $\mathrm{K}_{2}$ is estimated after the best possible values of the parameters in the extended CSBM are determined using data where adsorption density is relatively low: $\mathrm{K}_{2}$ is then calculated as follows: first, adsorption density from Modes I, II, and IV, which depend explicitly on the structure of the EDL and not on the presence of the second layer, is calculated; then, the resulting value of total adsorption density, $\Gamma_{S}^{\mathrm{I}+\mathrm{II}+\mathrm{IV}}$, is subtracted from the experimentally observed value. The remainder is attributed to second-layer adsorption, $\Gamma_{\mathrm{S}}^{\mathrm{III}}$. For cases where the model overpredicts the level of adsorption, due to experimental error or errors in the parameter estimates, the value of $\Gamma_{\mathrm{S}}^{\mathrm{III}}$ is set to zero. The quantity, $\mathrm{K}_{2} f_{\mathrm{S}}$, is then calculated from Eq. (47). For each system, the applicable value of $\mathrm{K}_{2} f_{\mathrm{S}}$ is calculated from the average of results for each individual data point.

Temperature effect. Since adsorption tends to decrease with temperature, the value of $\mathrm{K}_{2}$ is expected to decrease as well.

Structure effect. With decreasing tail length, $\mathrm{K}_{2}$ should increase to compensate for the reduced hydrophobic effect. In other words, to achieve the same level of second-layer coverage at two different tail lengths, $\mathrm{K}_{2}$ should be greater for the surfactant with the shorter tail.

$\mathrm{pH}$ and salinity effect. $\mathrm{K}_{2}$ is assumed to be independent of $\mathrm{pH}$, although this may be a poor assumption since second-layer coverage is expected to be very low at high values of $\mathrm{pH}$, where adsorption density is low. The effect of salinity on the equilibrium second-layer adsorption isotherm should be accounted for through the value of the surfactant activity coefficient, $f_{\mathrm{S}}$.

\section{Parameter_sensitivity}

The extended version of the CSBM is mathematically expressed as a system of seventeen nonlinear equations in which the parameters and variables are related in an extremely complicated way. For this reason, it is not a simple matter to understand the influence of a particular parameter on the predicted results. Before the parameters may be optimized with respect to the data, some insight into their effect is essential. This section is devoted to an examination of the sensitivity of the model results to values of $\mathrm{pK}_{\mathrm{S}}^{\mathrm{inn}}{ }^{*}$, 
$\mathrm{pK}_{\mathrm{S}}^{\mathrm{int}}{ }^{*}, \mathrm{~m}_{\mathrm{i}}$, and $\mathrm{n}$. A baseline case is presented for which these parameters have reasonable values. The results of changing one parameter value, while fixing the ralues of the others, are then compared to those calculated using the baseline case, to der onstrate the impact of the parameter value on the properties predicted by the model.

The parameters used in the baseline case are given in Table 1. The CSBM parameters to characterize a $\mathrm{TiO}_{2}$ surface are used here since the adsorption on this metal oxide is to be studied as a model system. The results of the calculations for each case are displayed in contour plots. In each figure, the first plot shows the results of the baseline calculation; the second plot shows the results of the comparison case. In all plots, $\theta_{1}$ is the sum of fractional coverage due to adsorption from Modes I, II, and IV; second-layer formation is not considered here. Because a wide range of values of $S_{\text {bulk }}^{-}$, expressed in $\mu \mathrm{M}$, and $\theta_{1}$ is covered, the values are expressed in terms of $\log _{10}\left(\mathrm{~S}_{\text {bulk }}^{-}\right)$and $\log _{10}\left(\theta_{1}+1\right)$ ( since the value of $\theta_{1}$ is sometimes negative, the constant 1 is added to insure that the argument of the $\log _{10}$ function is never negative).

Figure 3(a) shows contour levels of constant values of $\log _{10}\left(\theta_{1}+1\right)$ plotted as a function of $\mathrm{pH}$ and $\log _{10}\left(\mathrm{~S}_{\text {bulk }}^{-}\right)$for the baseline case at a background electrolyte concentration of $0.001 \mathrm{M} \mathrm{NaCl}$. Two major trends are evident. Coverage decreases with increasing $\mathrm{pH}$, as the electrostatic attraction between the surfactant ion and the the $\mathrm{TiO}_{2}$ surface decreases. Coverage increases with increasing $S_{\text {bulk }}^{-}$in response to the increasing chemical potential of the surfactant in the bulk solution.

Figure $3(\mathrm{~b})$ shows the effect of decreasing $\mathrm{pK}_{\mathrm{S}}^{\text {int }}{ }^{*}$ from 6.1 to 5.7. Comparison to the baseline results clearly show that adsorption density decreases for all values of $\mathrm{pH}$ and $\mathrm{S}_{\text {bulk }}^{-}$, because this change in the parameter value expresses a decrease in the affinity of the surfactant ion for the surface. This effect is observed for all levels of salinity.

Figure 4 shows the effect of decreasing $\mathrm{pK}_{\mathrm{SC}}^{\mathrm{int}^{*}}$ from 12.2 to 11.2 , which expresses a decrease in the tendency for clusters to form. At very low coverage, there is no effect, because adsorption occurs primarily due to formation of $\mathrm{MOH}_{2}^{+} \mathrm{S}^{-}$as expressed by $\mathrm{pK}_{\mathrm{S}}^{\mathrm{int}^{*}}$; but as $\mathrm{MOH}_{2}^{+} \mathrm{S}^{-}$increases, adsorption density decreases relative to the baseline case since cluster formation is less favored.

Figure 5 shows the effect of decreasing $\mathrm{m}_{\mathrm{i}}$ from 6 to 3 . This means that fewer methyl groups are interacting between the adsorbed molecules, so the net lateral interaction energy is decreased. At very low coverage, there is no effect, because there the molecules are too dilute on the surface to interact. As $\mathrm{S}_{\text {bulk }}^{-}$increases at constant $\mathrm{pH}$, however, a much lower level of coverage is predicted for the case where $m_{i}$ is lower. 
Figure 6 shows the effect of increasing the coordination number in the surfactant cluster from 2 to 2.5. This means that more $\mathrm{MOH}_{2}^{+} \mathrm{S}^{-}$groups must form before a neutral surfactant ion-counterion pair will adsorb. At low $\mathrm{pH}$ and low $\mathrm{S}_{\text {bulk }}^{-}$, there is no effect, again since adsorption is dominated by the formation of $\mathrm{MOH}_{2}^{+} \mathrm{S}^{-}$groups. As equilibrium surfactant concentration increases at constant $\mathrm{pH}$, adsorption density decreases significantly since neutral-pair adsorption is discouraged relative to the baseline case. With increasing $\mathrm{pH}$ at constant $\mathrm{S}_{\text {bulk }}^{-}$, coverage also decreases. This occurs since the formation of $\mathrm{MOH}_{2}^{+} \mathrm{S}^{-}$groups decreases with increasing $\mathrm{pH}$, resulting in less complexes available for cluster formation.

Figure 7(a) illustrates results of the baseline case at a background electrolyte concentration of $0.1 \mathrm{M} \mathrm{NaCl}$. Comparison with the baseline case at $0.001 \mathrm{M} \mathrm{NaCl}$ shows that, at low $\mathrm{pH}$ for very low coverages, increasing the salinity impedes adsorption. With more $\mathrm{Cl}^{-}$present, there is more competition with the surfactant ions for positively charged surface sites. Coverage is too low for lateral interaction energy to enhance adsorption, so coverage is decreased. Once adsorption begins, however, neutral-pair adsorption contributes significantly more than in the base case since more counterions are present, so adsorption density reaches the limiting level of monolayer coverage at a much lower value of $\mathrm{S}_{\text {bulk }}^{-}$. This effect is seen throughout the range of $\mathrm{pH}$ values.

Like Fig. 5, Fig. 7(b) shows the effect of decreasing $\mathrm{pK}_{\mathrm{SC}}^{\mathrm{int}}$ from 12.2 to 11.2, but $(\mathrm{NaCl})=0.1 \mathrm{M}$ in this case. The effect is a net decrease in adsorption density for all combinations of $\mathrm{pH}$ and $\mathrm{S}_{\text {bulk }}^{-}$. This is observed because cluster formation is a strong function of salinity, so changes in $\mathrm{pK}_{\mathrm{SC}}^{\mathrm{int}^{*}}$ have a profound effect on adsorption at high salinity.

Figure 8 shows the effect of increasing $\mathrm{n}$ from 2.0 to 2.5 for adsorption at $0.1 \underline{\mathrm{M}}$ $\mathrm{NaCl}$. The trends are the same as those observed for $0.001 \mathrm{M} \mathrm{NaCl}$ in Fig. 6, but magnified significantly due to the increased influence of salinity. Since fewer $\mathrm{MOH}_{2}^{+} \mathrm{S}^{-}$ groups form at high salinity relative to low salinity due to competition with $\mathrm{Cl}^{-}$, even a slight increase in $\mathrm{n}$ decreases adsorption at the same $\mathrm{pH}$ and surfactant concentration dramatically.

Figure 9(a) shows cintour levels of constant values of $\log _{10}\left(\theta_{1}+1\right)$ plotted as a function of $\log _{10}\left(\mathrm{~S}_{\text {bulk }}^{-}\right)$and surface charge density for the baseline case at a background electrolyte concentration of $0.001 \underline{\mathrm{M} \mathrm{NaCl}}$. Two significant trends are evident. As adsorption density increases, $\sigma_{\mathrm{O}}$ increases at a constant level of $\mathrm{S}_{\text {bulk }}^{-}$. This is attributable to the increase in concentration of $\mathrm{MOH}_{2}^{+} \mathrm{S}^{-}$groups formed. Consider the behavior of a 
contour of constant adsorption density: with increasing surfactant concentration, the same level is achieved at a lower value of $\sigma_{\mathrm{O}}$. This occurs due to cluster formation accompanied by adsorption of neutral pairs, which contribute to $\theta_{1}$ but not to $\sigma_{\mathrm{O}}$.

Figure 9 (b) shows the effect on surface charge density of decreasing $\mathrm{pK}_{\mathrm{SC}}^{\text {int }}$ from 12.2 to 11.2. Again, consider a line of constant adsorption density: as surfactant concentration increases, a higher value of $\sigma_{\mathrm{O}}$ is required to maintain that level than in the base case. If neutral pairs are not accounted for in the contribution to the overall level of adsorption density, surface charge density is severely overpredicted.

The predicted values of surface charge density and zeta potential also change in a systematic fashion with changes in the parameters. Because they are consistent and not surprising, these trends may be qualitatively discussed. Any change in parameters which promotes the formation of $\mathrm{MOH}_{2}^{+} \mathrm{S}^{-}$groups at a particular solution composition relative to that of the baseline case causes an attendant increase in the value of surface charge density. Any increase in affinity of the surfactant ion for the surface relative to its counterion in solution shifts the IEP downward; so at a given level of $\mathrm{pH}$, any reduction in the tendency for adsorption to occur requires a higher value of $S_{\text {bulk }}^{-}$to achieve charge reversal at the OHP. The effect of greater affinity of the surfactant ion for the surface relative to that of $\mathrm{C}^{+}$on both the PZC and the IEP is illustrated in Fig. 10. With increasing $\mathrm{S}_{\text {bulk }}^{-}$, reflected in increasing adsorption density, the IEP shifts to lower values of $\mathrm{pH}$ (Fig. 10(a)), while the PZC shifts to higher values of $\mathrm{pH}$ (Fig. 10(b)).

\section{Summary}

A new model of adsorption of ionic surfactant at the metal oxide/aqueous solution interface has been proposed. It incorporates a detailed description of the electrical double layer which has been shown to successfully predict the major trends in experimentally derived values of surface charge density and zeta potential for a wide range of $\mathrm{pH}$ and ionic strength on a variety of oxides. This model has been extended to describe the adsorption of ionic surfactant in the electrical double layer in several ways. Surfactant ions may complex at the surface due to electrostatic attraction; or they may adsorb there as neutral surfactant ion-counterion pairs if the surface environment is favorable. Electrostatic interaction of the surfactant ions with the OHP is also accounted for in this model. On the basis of this extension of the CSBM, given values of the new parameters which have been introduced, together with values of the parameters which describe the solid/solution-interface in the absence of surfactant, adsorption density, surface charge density, and zeta potential may all be predicted as a function of $\mathrm{pH}$, salinity, temperature, and structure. At high levels of 
surface coverage, the possibility of formation of a second layer of surfactant on top of the first layer has been postulated. It has been added in an ad hoc sense due to simplifications in the complete model required to make the solution of the model equations tractable.

The parameters required by the new model must assume reasonable values which change in a systematic fashion with changes in system variables. The ultimate test of the validity of this viewpoint is the successful prediction with a reasonable set of parameters of not just adsorption density, but surface charge density anc zeta potential, for several different systems over a wide range of solution conditions. This validation of the model is now being carried out. 
Table 1. Baseline Case for Parameter Sensitivity Study (Values are Typical of $\mathrm{TiO}_{2}$ ).

\begin{tabular}{|c|c|c|c|c|}
\hline $\mathrm{pK}_{\mathrm{S}}^{\mathrm{int}^{*}}$ & $\mathrm{pK}_{\mathrm{SC}}^{\mathrm{int}}$ & $\mathrm{m}_{\mathrm{i}}$ & $n$ & $\mathrm{~T},{ }^{\circ} \mathrm{C}$ \\
\hline 6.1 & 12.2 & 6 & 2.0 & 30 \\
\hline
\end{tabular}




\section{List of Figures}

Figure

1 Illustration of surfactant adsorption by complexation (Mode I) and clustering (Mode $\mathrm{I}$ ) at the IHP

2 Schematic diagram of compretensive model of ionic surfactant adsorption at the metal oxide/aqueous solution interface

3(a) Contours of constant adsorption density as a function of $\mathrm{pH}$ and $\mathrm{S}_{\mathrm{bulk}}^{-}$in $0.001 \mathrm{M} \mathrm{NaCl}$ at $30^{\circ} \mathrm{C}$. Baseline adsorption model parameters $\mathrm{pK}_{\mathrm{S}}^{\text {int* }}=6.1$ $\mathrm{pK}_{\mathrm{SC}}^{\mathrm{int}}=12.2 \mathrm{~m}_{\mathrm{i}} \omega=6.0 \mathrm{n}=2.0$

3(b) $\quad$ Effect on adsorption density of decreasing $\mathrm{pK}_{\mathrm{S}}^{\mathrm{int}}: \mathrm{pK}_{\mathrm{S}}^{\mathrm{int}{ }^{*}}=5.7$

4(a) Contours of constant adsorption density as a function of $\mathrm{pH}$ and $\mathrm{S}_{\mathrm{bulk}^{-}}^{-}$in $0.001 \mathrm{M} \mathrm{NaCl}$ at $30^{\circ} \mathrm{C}$. Baseline adsorption model parameters $\mathrm{pK}_{\mathrm{S}}^{\text {int* }} \stackrel{\text { bulk }}{=6.1}$ $\mathrm{pK}_{\mathrm{SC}}^{\text {int }}=12.2 \mathrm{~m}_{\mathrm{i}} \omega=6.0 \mathrm{n}=2.0$

4(b) Effect on adsorption density of decreasing $\mathrm{pK}_{\mathrm{SC}}^{\mathrm{int}}: \mathrm{pK}_{\mathrm{SC}}^{\mathrm{int}}=11.2$

5(a) Contours of constant adsorption density as a function of $\mathrm{pH}$ and $\mathrm{S}_{\mathrm{bulk}}^{-}$in $0.001 \underline{\mathrm{M} \mathrm{NaCl}}$ at $30^{\circ} \mathrm{C}$. Baseline adsorption model parameters $\mathrm{pK}_{\mathrm{S}}^{\text {int* }}{ }^{\mathrm{bull}^{*}}=6.1$ $\mathrm{pK}_{\mathrm{SC}}^{\text {int* }}=12.2 \mathrm{~m}_{\mathrm{i}} \omega=6.0 \mathrm{n}=2.0$

5(b) Effect on adsorption density of decreasing $m_{i} \omega: m_{i} \omega=3.0$

6(a) Contours of constant adsorption density as a function of $\mathrm{pH}$ and $\mathrm{S}_{\text {bulk }}^{-}$in $0.001 \mathrm{M} \mathrm{NaCl}$ at $30^{\circ} \mathrm{C}$. Baseline adsorption model parameters $\mathrm{pK}_{\mathrm{S}}^{\text {int* }}=6.1$ $\mathrm{pK}_{\mathrm{SC}}^{\mathrm{int}}=12.2 \mathrm{~m}_{\mathrm{i}} \omega=6.0 \mathrm{n}=2.0$

6(b) Effect on adsorption density of increasing $n: n=2.5$

7 (a) Contours of constant adsorption density as a function of $\mathrm{pH}$ and $\mathrm{S}_{\text {bulk }}^{-}$in $0.1 \mathrm{M} \mathrm{NaCl}$ at $30^{\circ} \mathrm{C}$. Baseline adsorption model parameters $\mathrm{pK}_{\mathrm{S}}^{\text {int* }}=6.1$ $\mathrm{pK}_{\mathrm{SC}}^{\text {int }}=12.2 \mathrm{~m}_{\mathrm{i}} \omega=6.0 \mathrm{n}=2.0$

7(b) Effect on adsorption density of decreasing $\mathrm{pK}_{\mathrm{SC}}^{\mathrm{int}}: \mathrm{pK}_{\mathrm{SC}}^{\mathrm{int}}=11.2$

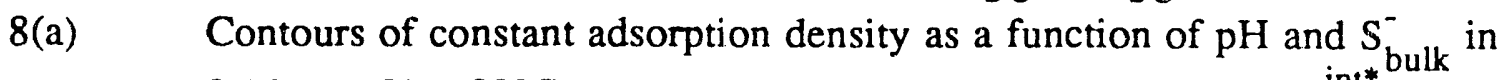
$0.1 \mathrm{M} \mathrm{NaCl}$ at $30^{\circ} \mathrm{C}$. Baseline adsorption model parameters $\mathrm{pK}_{\mathrm{S}}^{\text {int* }}=6.1$ $\mathrm{pK}_{\mathrm{SC}}^{\text {int }}=12.2 \mathrm{~m}_{\mathrm{i}} \omega=6.0 \mathrm{n}=2.0$.

8(b) Effect on adsorption density of increasing $n: n=2.5$

9(a) Contours of constant adsorption density as a function of $\mathrm{S}_{\text {bulk }}^{-}$and surface charge density in $0.001 \mathrm{M} \mathrm{NaCl}$ at $30^{\circ} \mathrm{C}$. Baseline adsorption model parameters $\mathrm{pK}_{\mathrm{S}}^{\text {int* }}=6.1 \mathrm{pK}_{\mathrm{SC}}^{\mathrm{int}}=12.2 \mathrm{~m}_{\mathrm{i}} \omega=6.0 \mathrm{n}=2.0$ 
9(b) Effect on surface charge density at constant levels of adsorption density of decreasing $\mathrm{pK}_{\mathrm{SC}}^{\mathrm{int}}: \mathrm{pK}_{\mathrm{SC}}^{\mathrm{int}}=11.2$

10(a) Contours of constant adsorption density as a function of $\mathrm{pH}$ and surface charge density at $0.001 \mathrm{M} \mathrm{NaCl}$ at $30^{\circ} \mathrm{C}$ to show effect of unequal ion affinity on IEP

10(b) Contours of constant adsorption density as a function of $\mathrm{pH}$ and zeta potential in $0.001 \mathrm{M} \mathrm{NaCl}$ at $30^{\circ} \mathrm{C}$ to show effect of unequal ion affinity on PZC. Baseline adsorption model parameters $\mathrm{pK}_{\mathrm{S}}^{\mathrm{int}}=6.1 \mathrm{pK}_{\mathrm{SC}}^{\mathrm{int}}=12.2$ $m_{i} \omega=6.0 n=2.0$ were used to generate bothı figures 


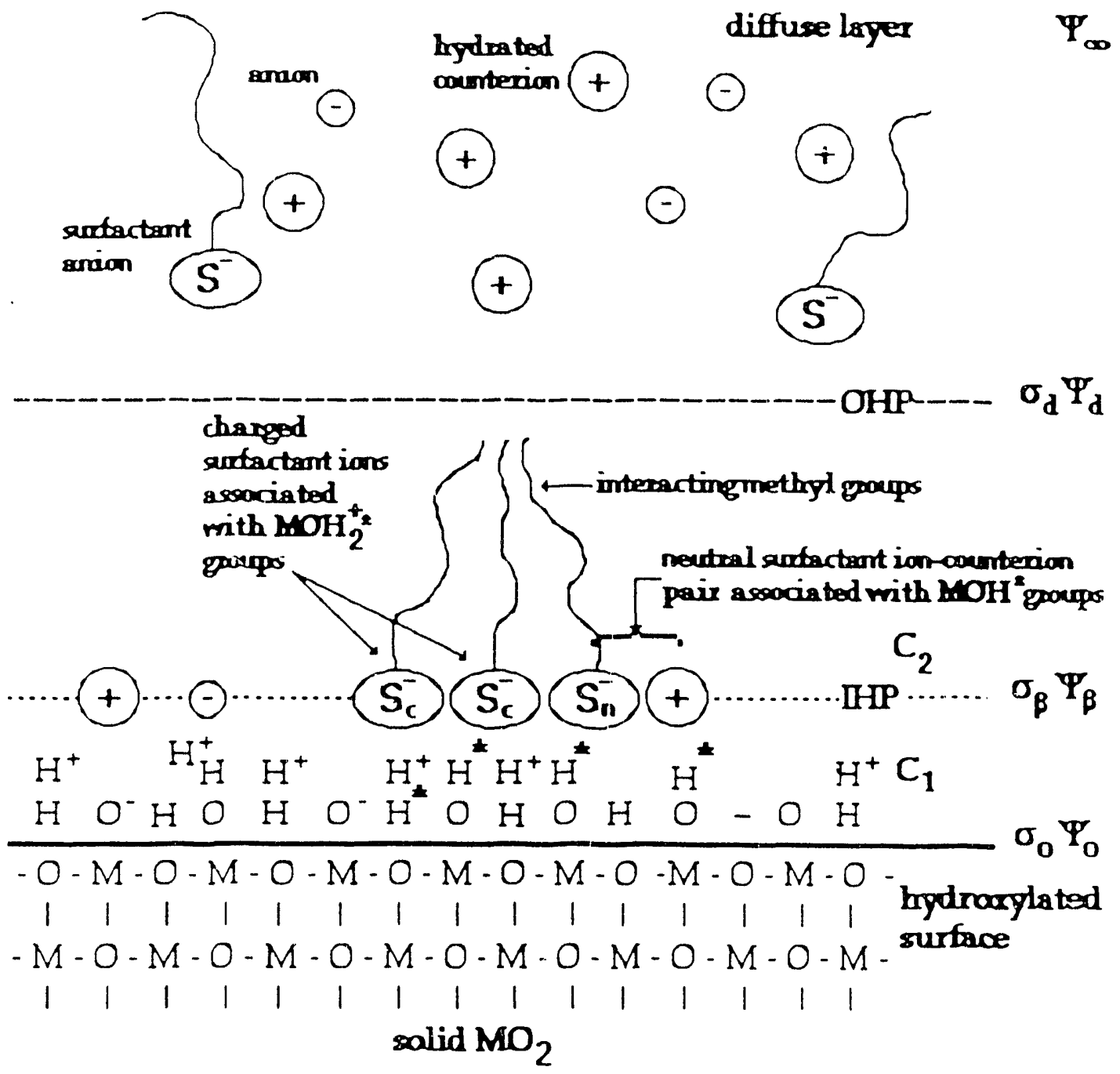

Figure 1. Illustration of surfactant adsorption by complexation (Mode I) and clustering (Mode II) at the IHP. 


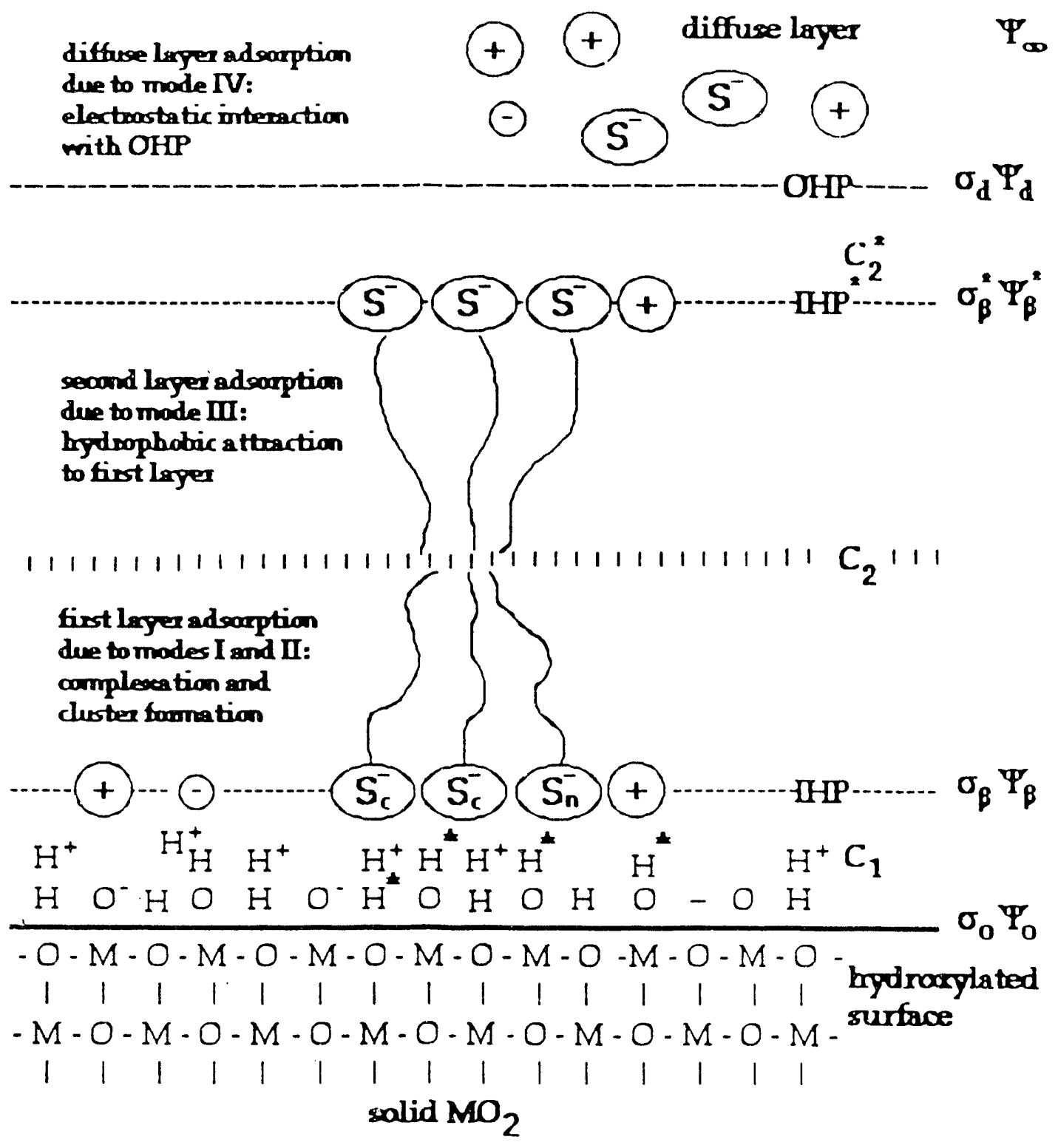

Figure 2. Schematic diagram of comprehensive model of ionic surfactant adsorption at the metal oxide/aqueous solution interface. 


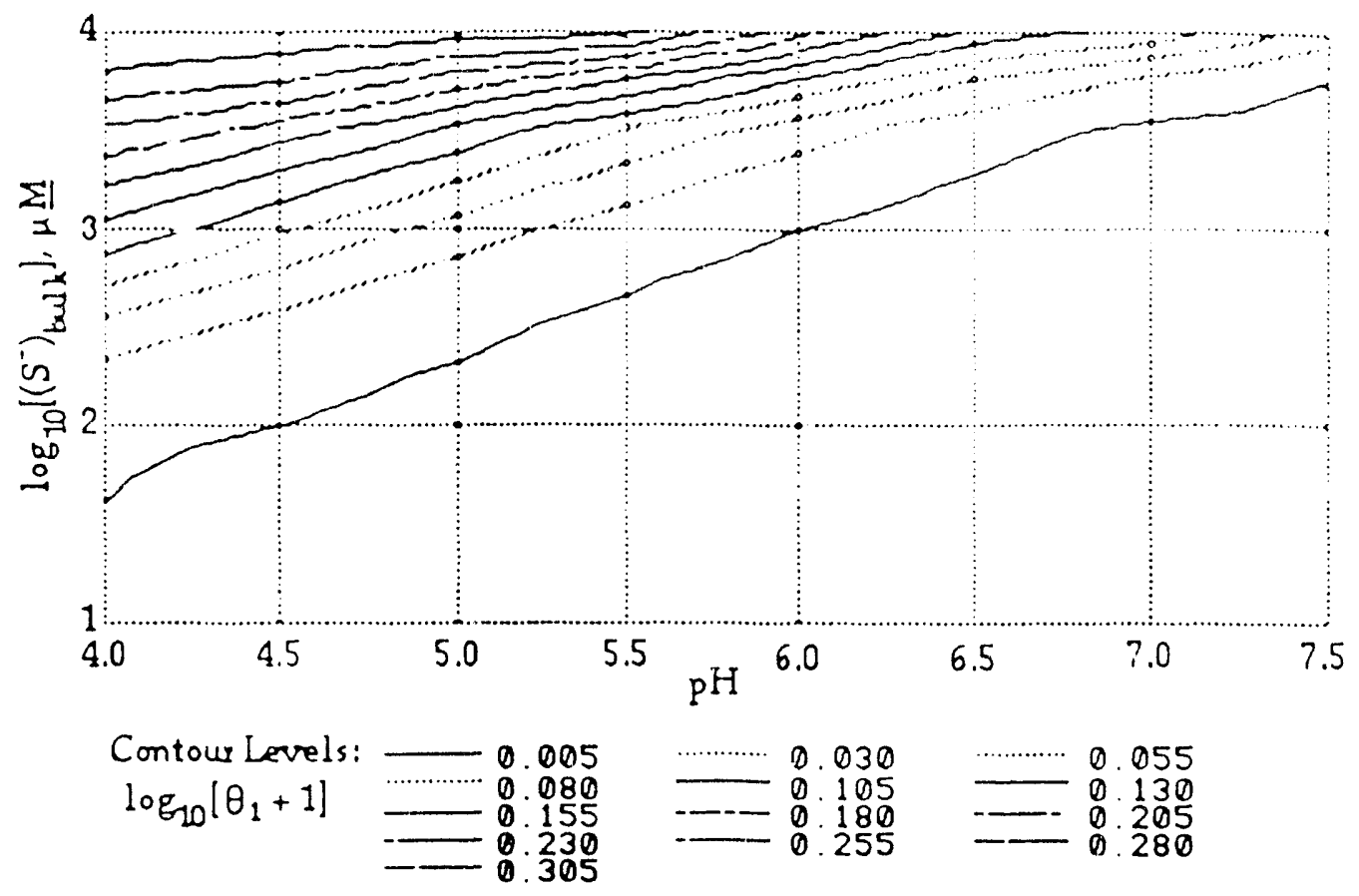

Figure 3(a). Contours of constant adsorption density as a function of $\mathrm{pH}$ and $\mathrm{S}_{\text {bulk }}^{-}$in $0.001 \mathrm{M} \mathrm{NaCl}$ at $30^{\circ} \mathrm{C}$. Baseline adsorption model parameters $\mathrm{pK}_{\mathrm{S}}^{\mathrm{int^{* }}}=6.1 \mathrm{pK}_{\mathrm{SC}}^{\mathrm{int}}=12.2 \mathrm{~m}_{\mathrm{i}} \omega=6.0 \mathrm{n}=2.0$.

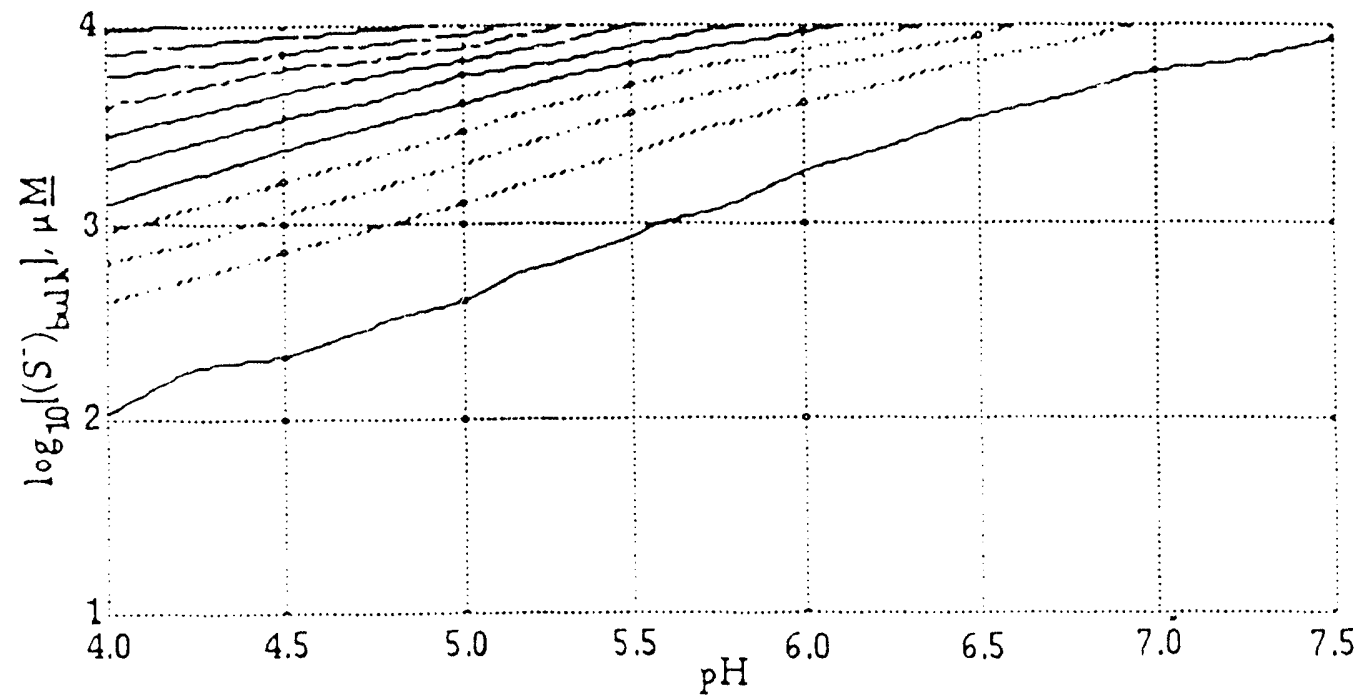

Figure 3(b). Effect on adsorption density of decreasing $\mathrm{pK}_{\mathrm{S}}^{\mathrm{int}}: \mathrm{pK}_{\mathrm{S}}^{\mathrm{int}}=5.7$. 


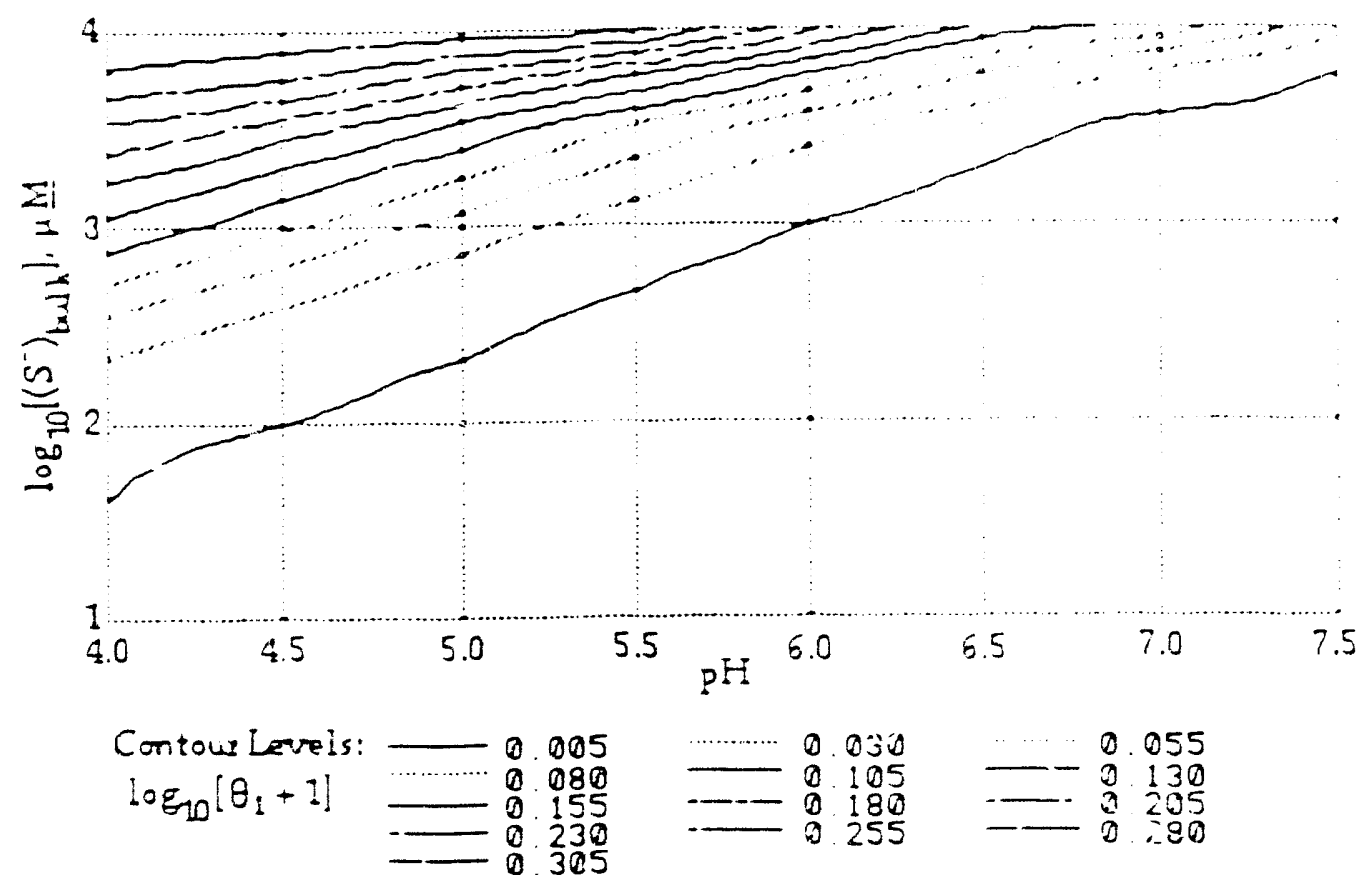

Figure 4(a). Contours of constant adsorption density as a function of $\mathrm{pH}$ and $\mathrm{S}_{\text {bulk }}^{-}$in $0.001 \mathrm{M} \mathrm{NaCl}$ at $30^{\circ} \mathrm{C}$. Baseline adsorpuon model farameters $\mathrm{pK}_{\mathrm{S}}^{\mathrm{int^{* }}}=5.1 \mathrm{pK}_{\mathrm{SC}}^{\mathrm{int*}}=12.2 \mathrm{~m}_{\mathrm{i}}(\omega)=6.0 \mathrm{n}=2.0$.

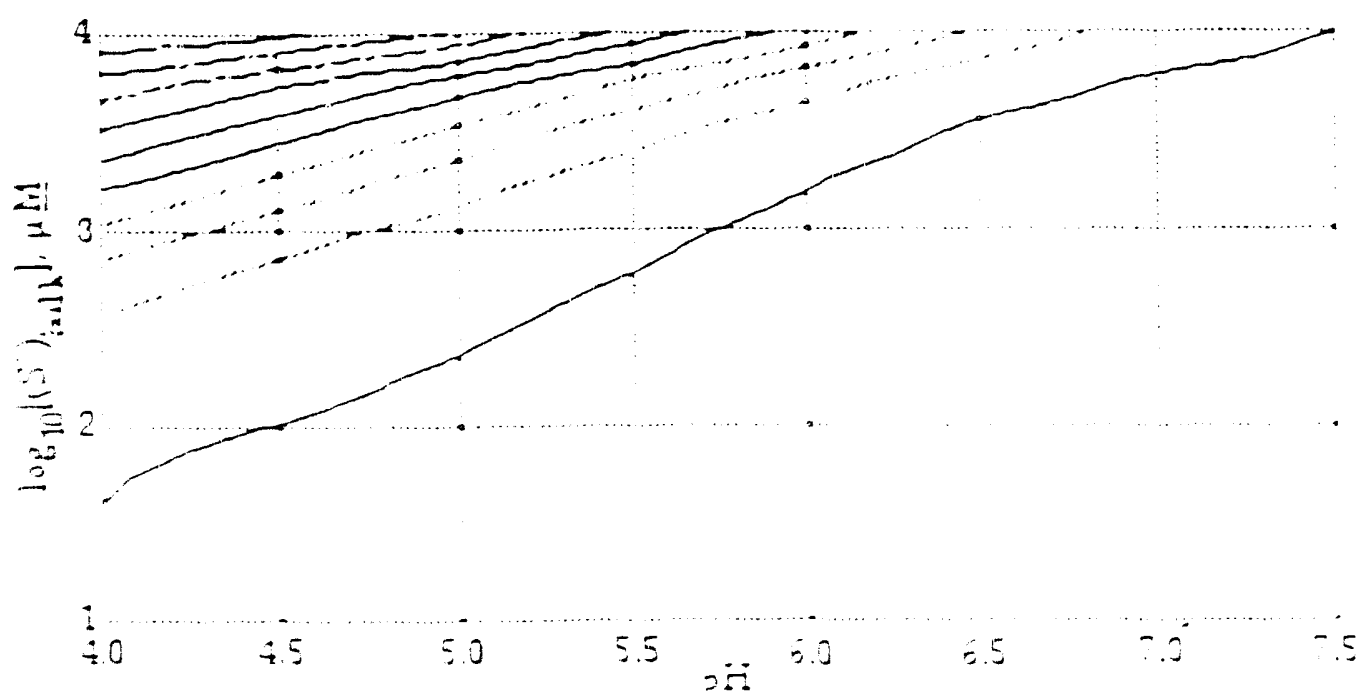

Eigure $+(b)$ Effect on adsorption density of decreasing $p K_{S C}^{\ln t^{*}}: p K_{S C}^{i n t^{*}}=11.2$. 


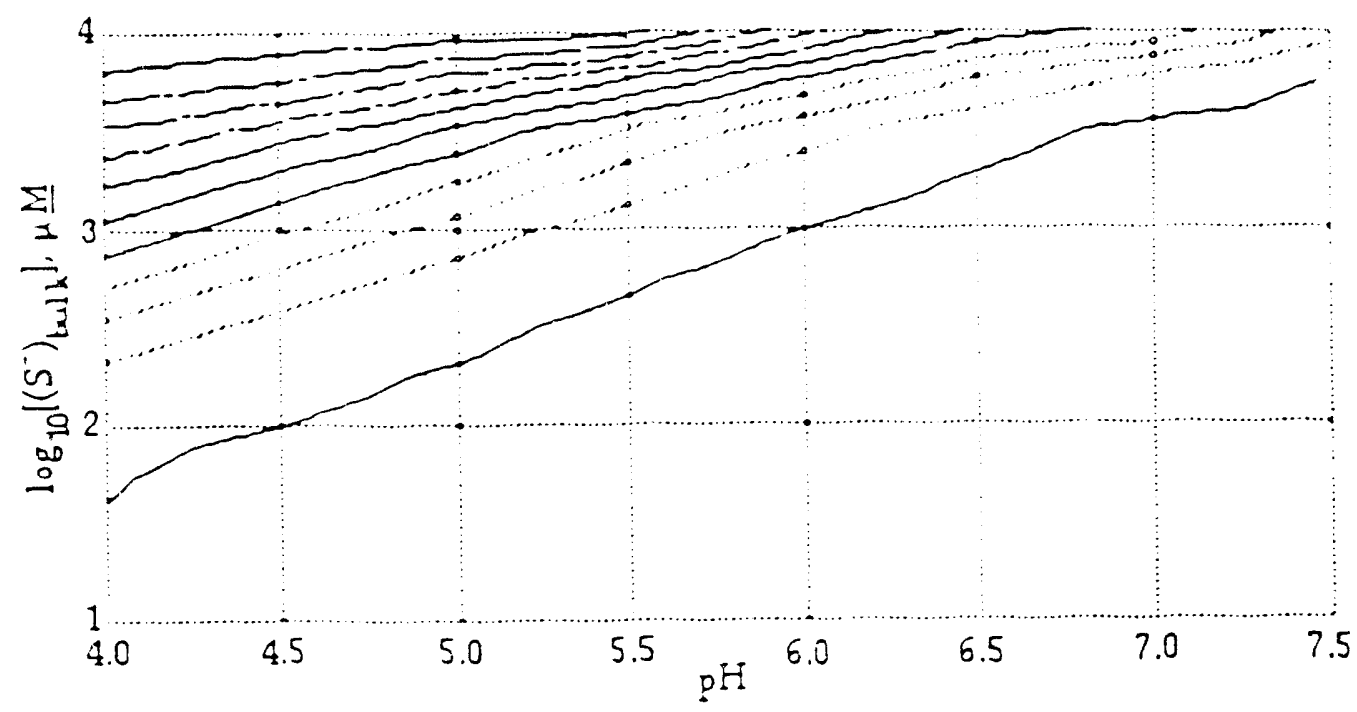

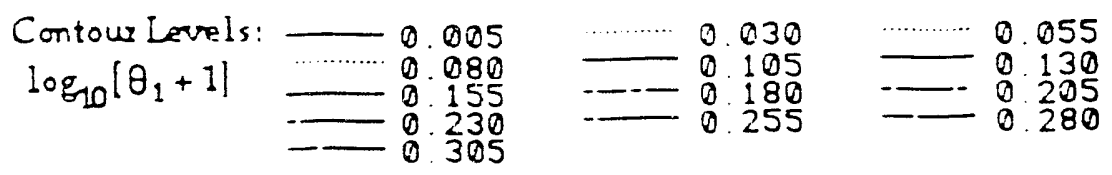

Figure 5(a). Contours of constant adsorption density as a function of $\mathrm{pH}$ and $\mathrm{S}_{\text {bulk }}^{-}$in $0.001 \mathrm{M} \mathrm{NaCl}$ at $30^{\circ} \mathrm{C}$. Baseline adsorption model parameters $\mathrm{pK}_{\mathrm{S}}^{\text {int* }}=6.1 \mathrm{pK}_{\mathrm{SC}}^{\mathrm{int^{* }}}=12.2 \mathrm{~m}_{\mathrm{i}} \omega=6.0 \mathrm{n}=2.0$.

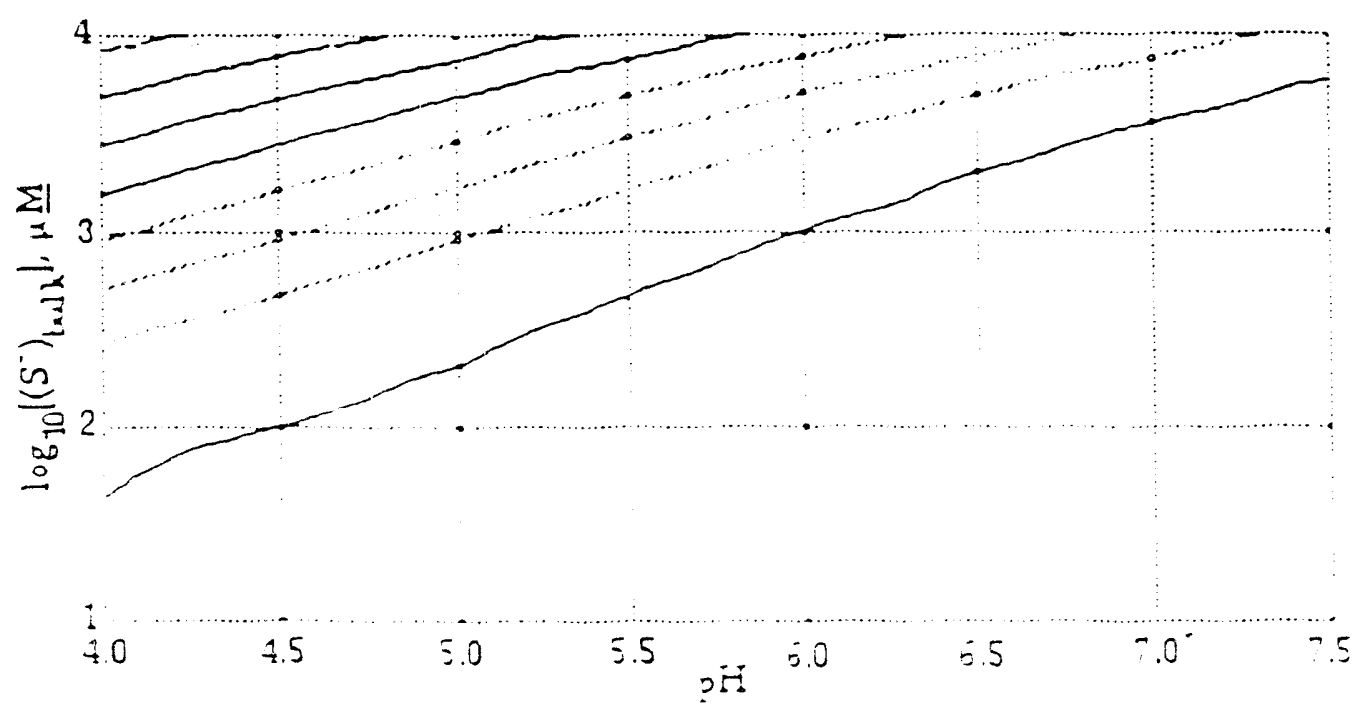

Figure $\Sigma(b)$. Effect on adsorption density of decreasing $m \omega: m_{i} \omega=3.0$. 


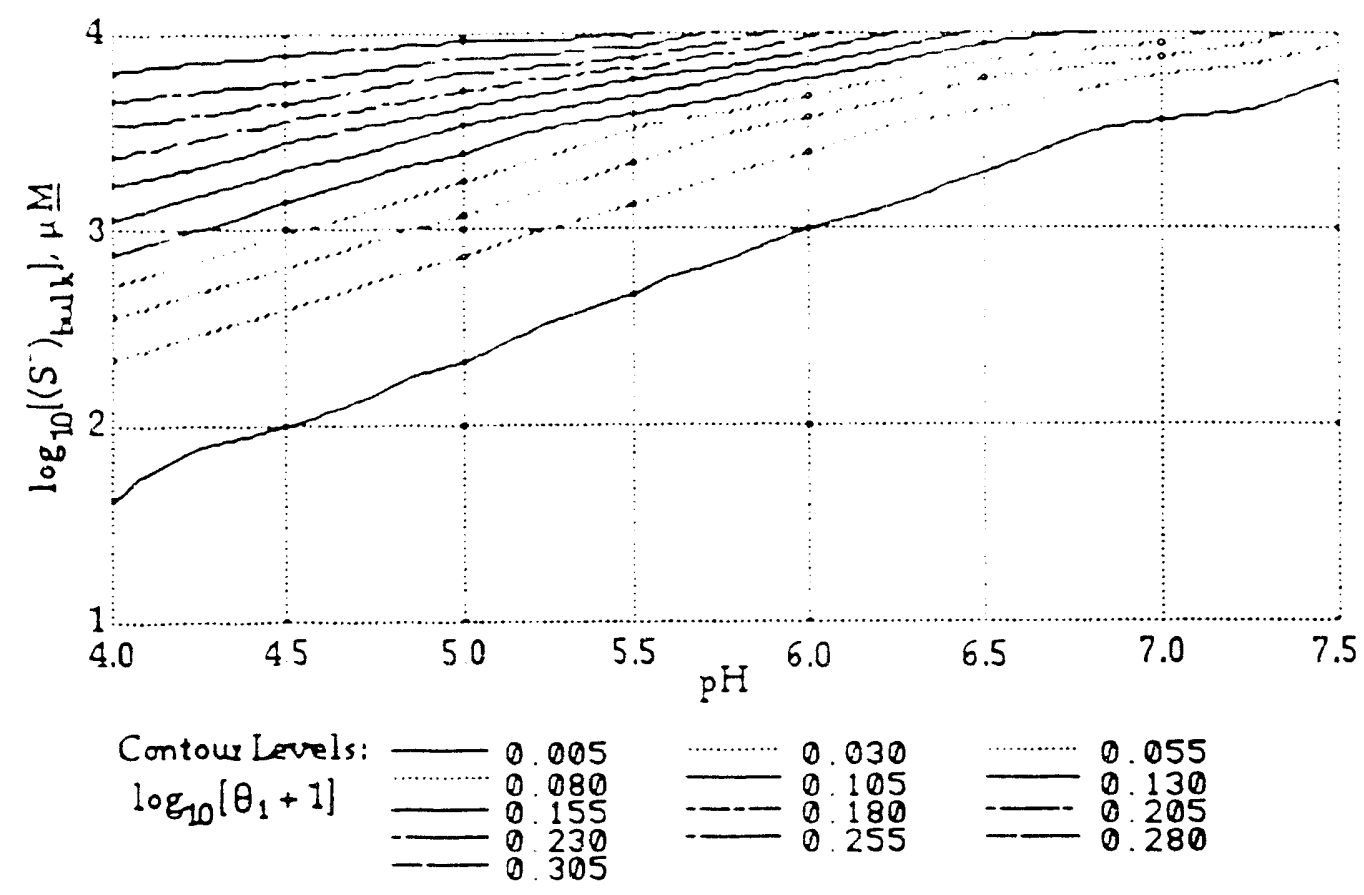

Figure 6(a). Contours of constant adsorption density as a function of $\mathrm{pH}$ and $\mathrm{S}_{\text {bulk }}^{-}$in $0.001 \mathrm{M} \mathrm{NaCl}$ at $30^{\circ} \mathrm{C}$. Baseline adsorption model parameters $\mathrm{pK}_{\mathrm{S}}^{\text {int* }}=6.1 \mathrm{pK}_{\mathrm{SC}}^{\text {int* }}=12.2 \mathrm{~m}_{\mathrm{i}} \omega=6.0 \mathrm{n}=2.0$.

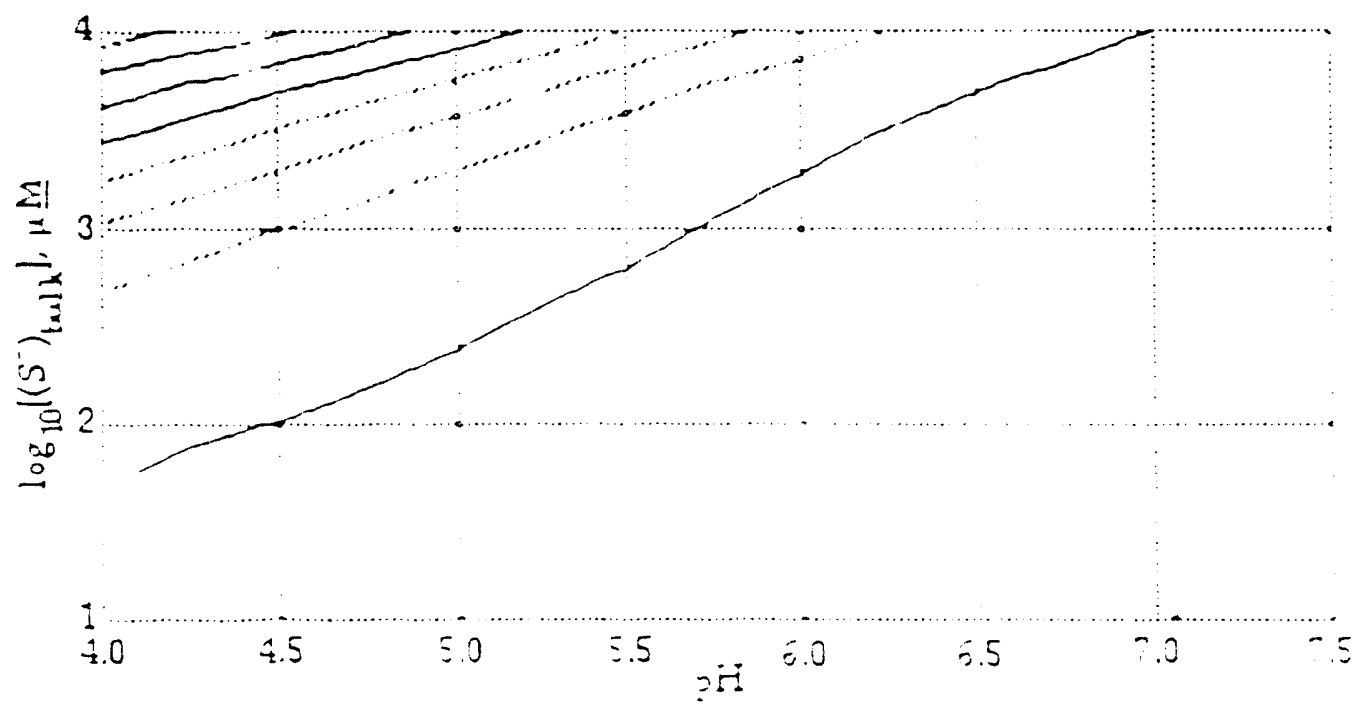

Figure $o(b)$. Effect on adsorption density of increasing $n: n=2.5$. 


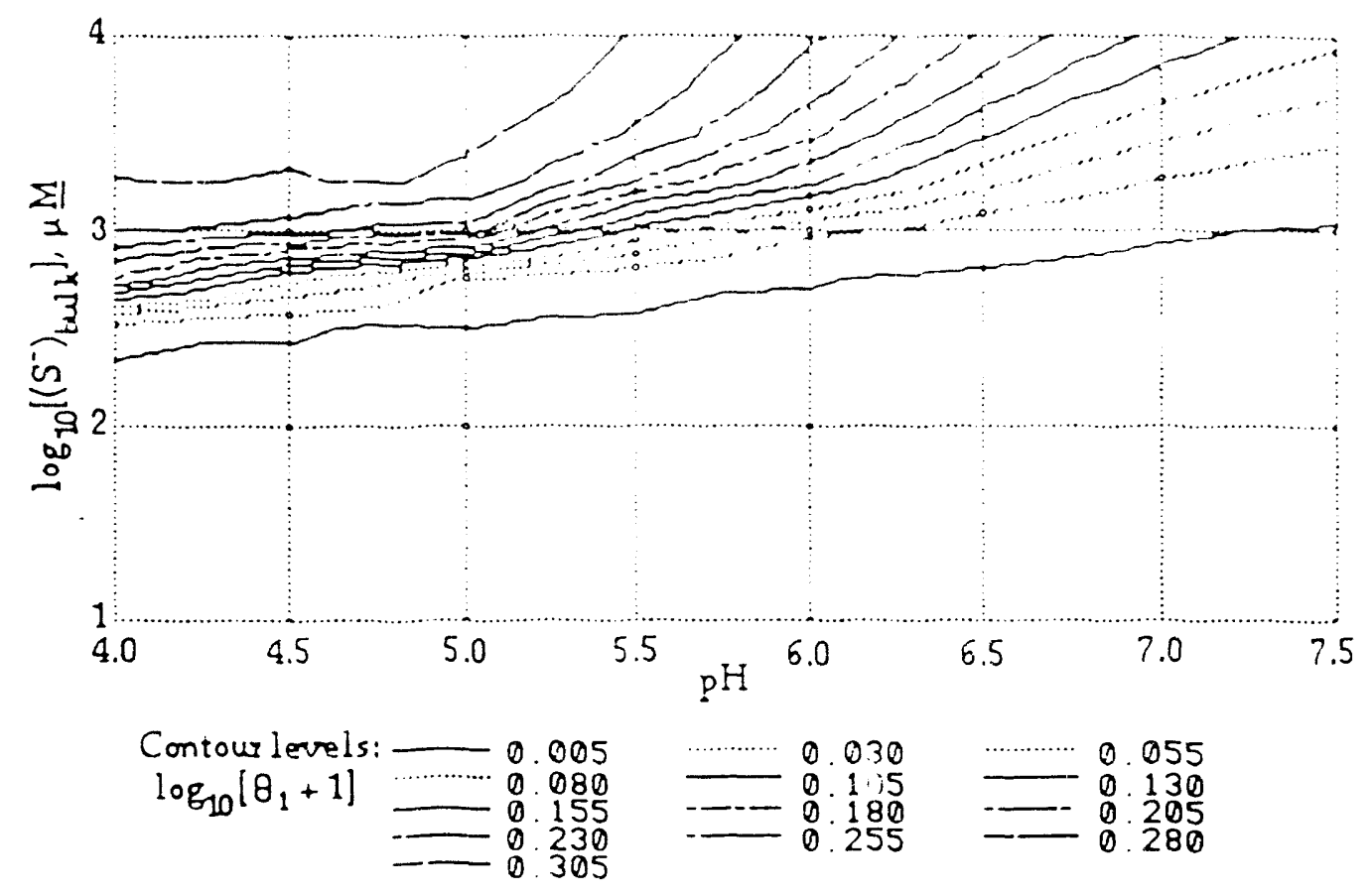

Figure 7(a). Contours of constant adsorption density as a function of $\mathrm{pH}$ and $\mathrm{S}_{\text {bulk }}^{-}$in $0.1 \underline{\mathrm{M} \mathrm{NaCl}}$ at $30^{\circ} \mathrm{C}$. Baseline adsorption model parameters $\mathrm{pK}_{S}^{\text {int* }}=6.1 \mathrm{pK}_{\mathrm{SC}}^{\mathrm{in} \iota^{*}}=12.2 \mathrm{~m}_{\mathrm{i}} \omega=6.0 \mathrm{n}=2.0$.

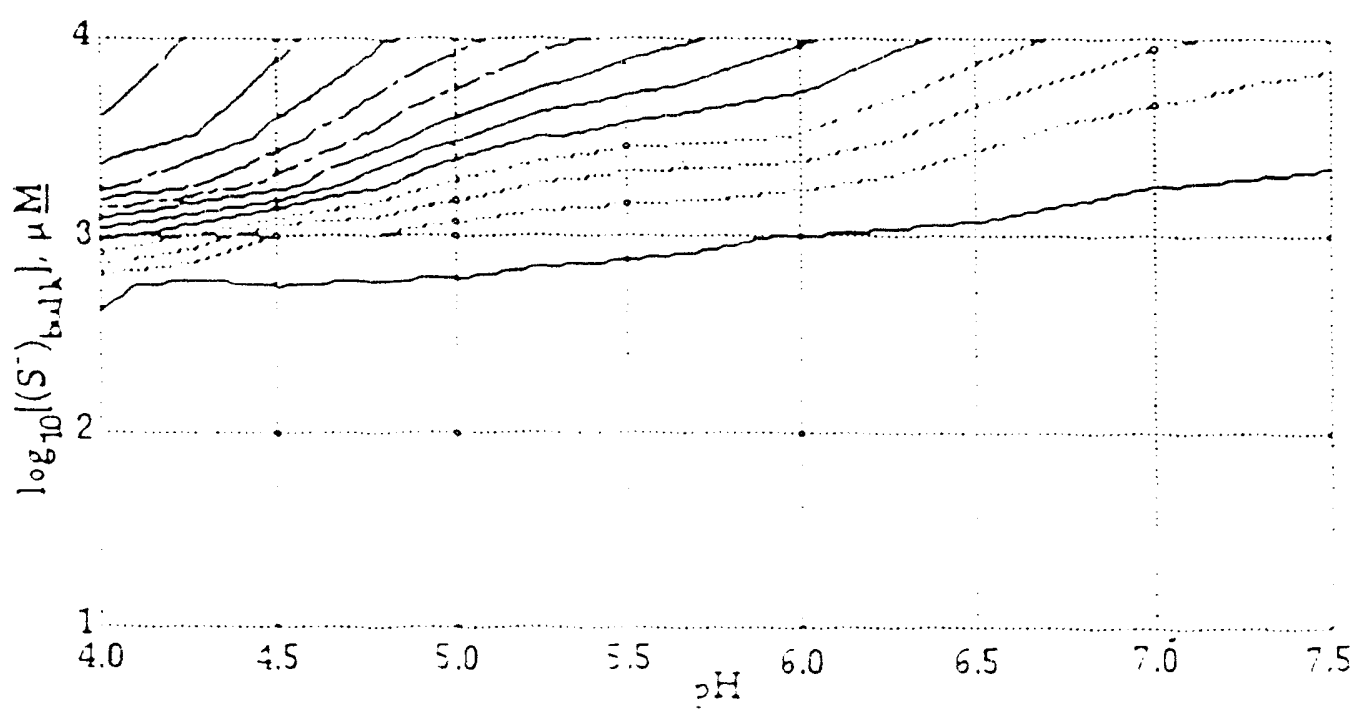

Figure $7(b)$ Effect on adsorption density of decreasing $\mathrm{pK}_{\mathrm{SC}}^{\mathrm{inl^{* }}:} \mathrm{pK}_{\mathrm{SC}}^{\mathrm{in} t^{*}}=11.2$. 


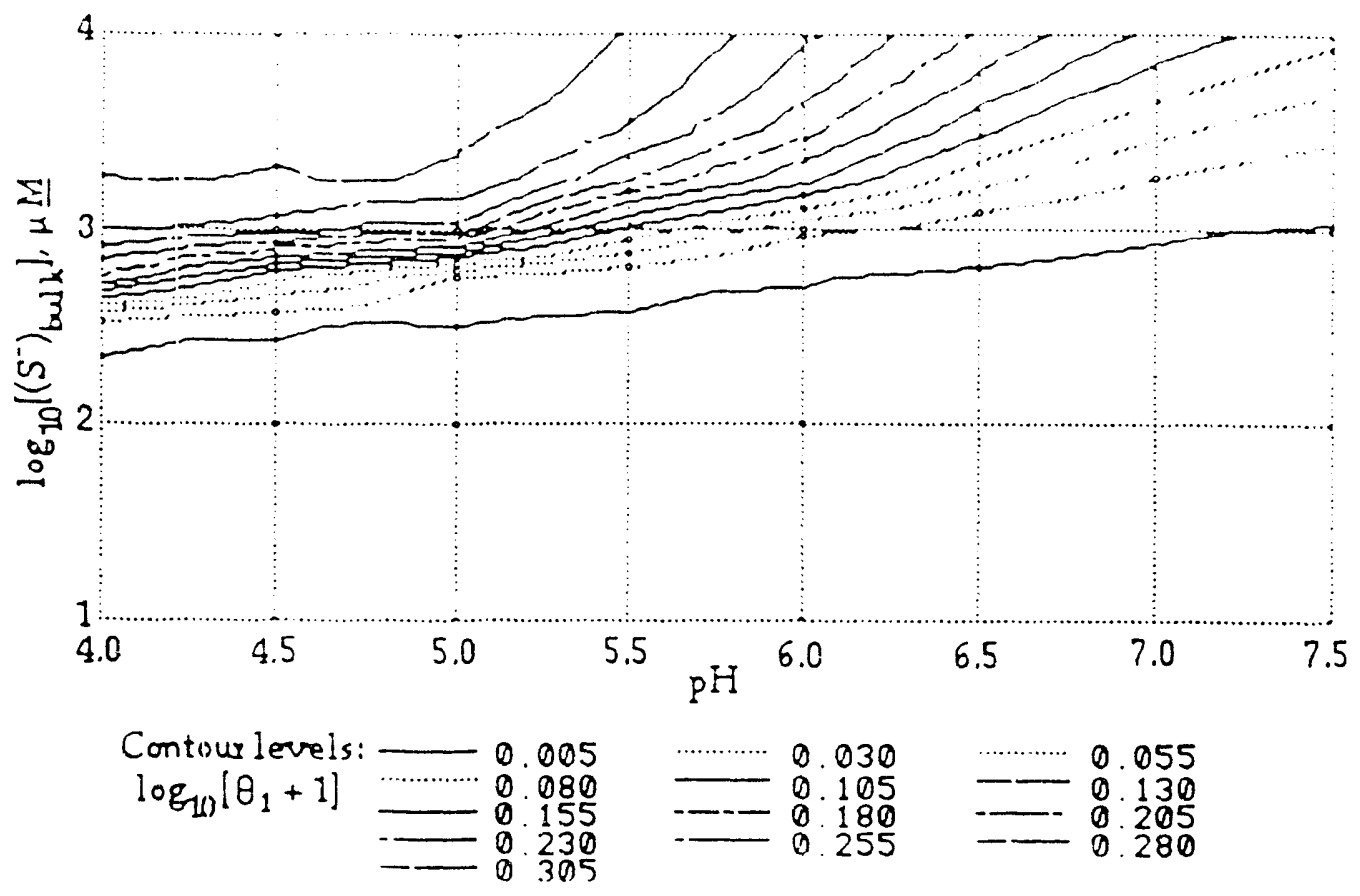

Figure 8(a). Contours of constant adsorption density as a function of $\mathrm{pH}$ and $\mathrm{S}_{\text {bulk }}^{-}$in $0.1 \mathrm{M} \mathrm{NaCl}$ at $30^{\circ} \mathrm{C}$. Baseline adsorption model parameters $\mathrm{pK}_{\mathrm{S}}^{\mathrm{int} *^{*}}=6.1 \mathrm{pK}_{\mathrm{SC}}^{\mathrm{int}}=12.2 \mathrm{~m}_{\mathrm{i}} \omega=6.0 \mathrm{n}=2.0$.

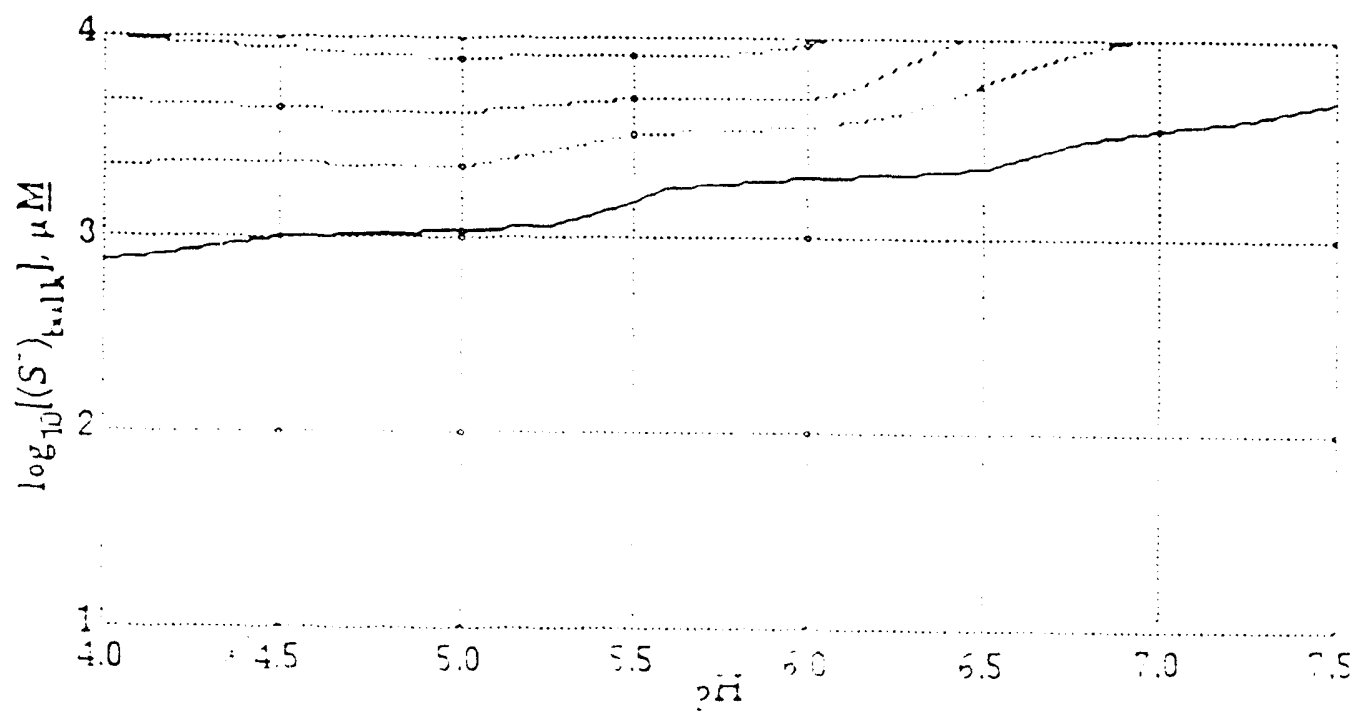

Figure $\mathrm{x}(\mathrm{b})$. Effect on adsorption density of increasing $\mathrm{n}: \mathrm{n}=2.5$. 


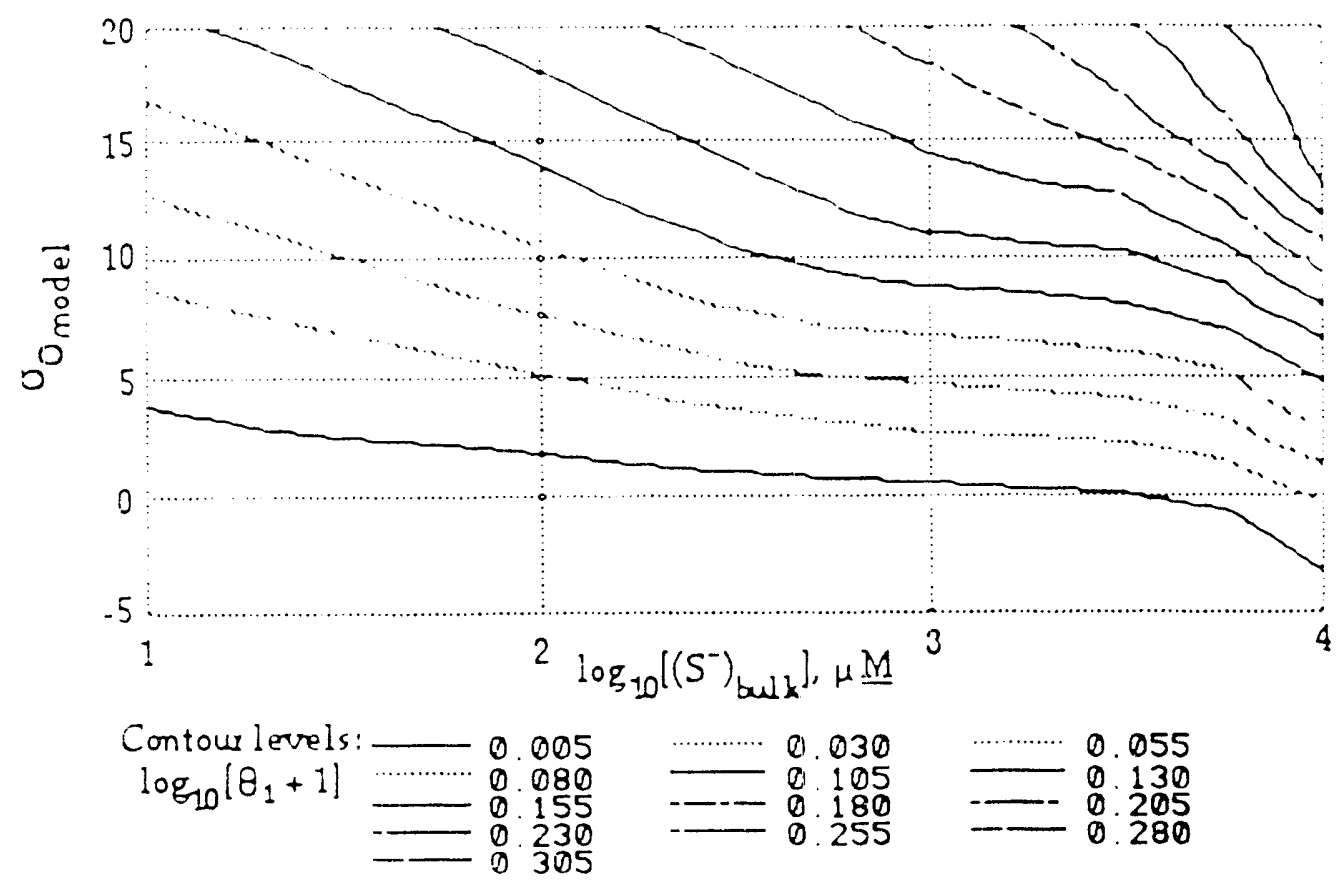

Figure 9(a). Contours of constant adsorption density as a function of $\mathrm{S}_{\text {bulk }}^{-}$and surface charge density in $0.001 \mathrm{M} \mathrm{NaCl}$ at $30^{\circ} \mathrm{C}$. Baseline adsorption model parameters $\mathrm{pK}_{\mathrm{S}}^{\text {in* }}$ $=6.1 \mathrm{pK}_{\mathrm{SC}}^{\mathrm{int}}=12.2 \mathrm{~m}_{\mathrm{i}} \omega=6.0 \mathrm{n}=2.0$.

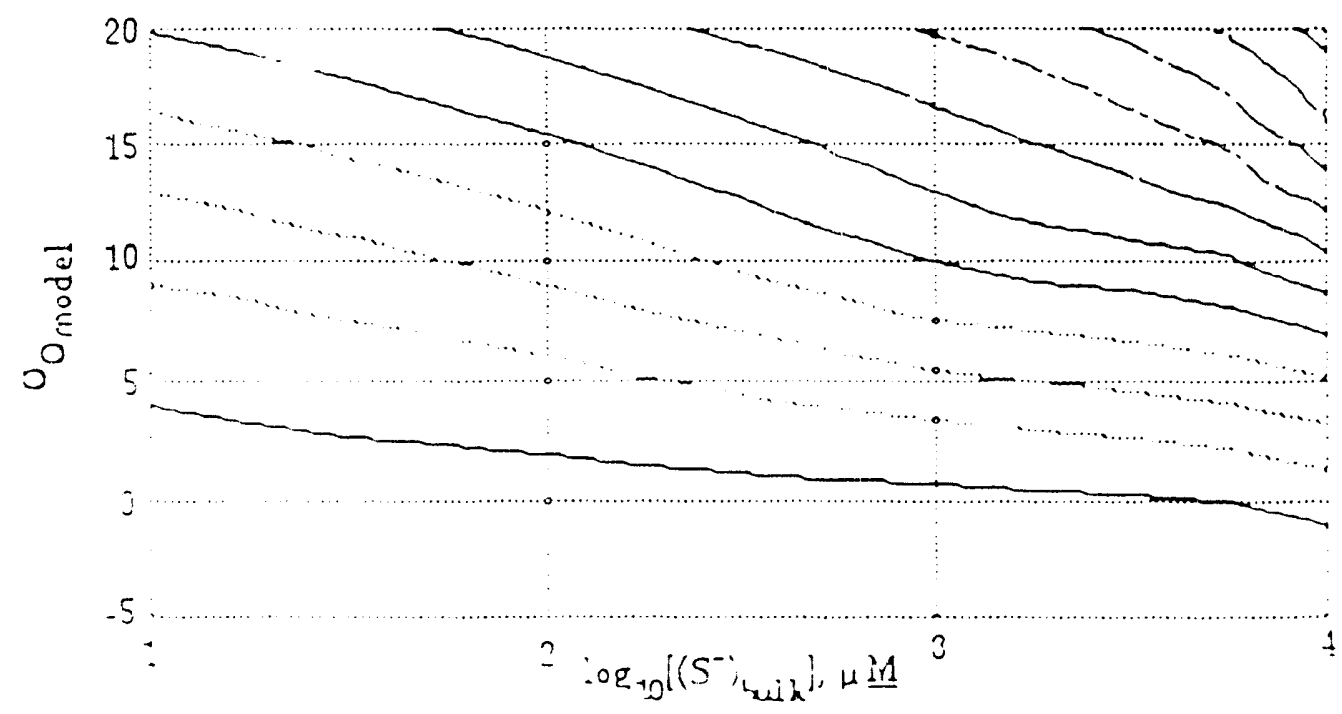

Figure $9(b)$. Effect on surface charge density at constant levels or

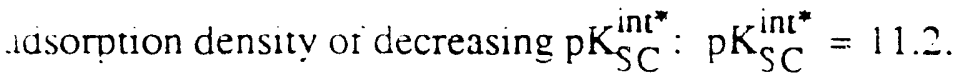




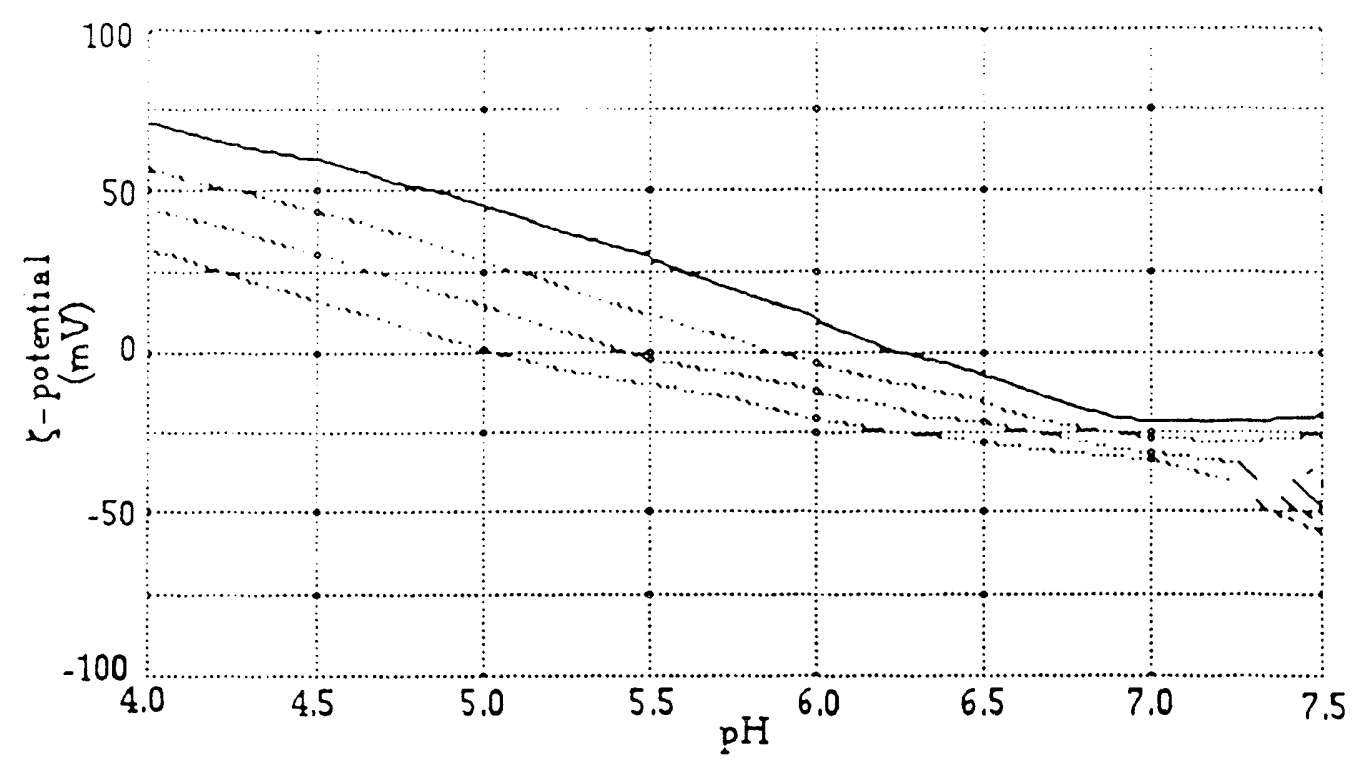

Figure 10(a). Contours of constant adsorption density as a function of $\mathrm{pH}$ and surface charge density at $0.001 \mathrm{M} \mathrm{NaCl}$ at $30^{\circ} \mathrm{C}$ to show effect of unequal ion affinity on IEP.

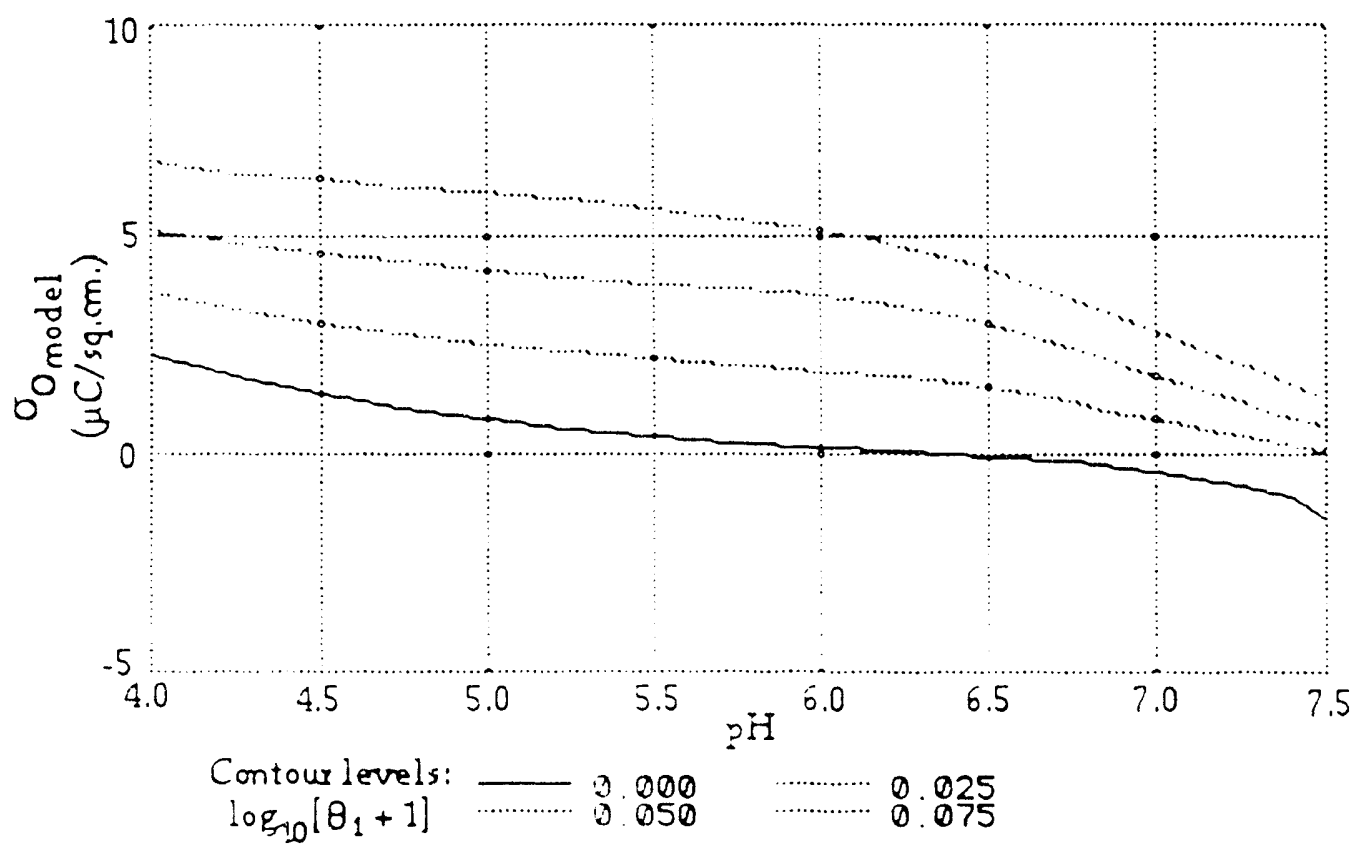

Figure 10(b). Contours of constant adsorption density as a function of $\mathrm{pH}$ and zeta potential in $0.001 \mathrm{M} \mathrm{NaCl}$ at $30^{\circ} \mathrm{C}$ to show effect of unequal ion affinity on PZC. Baseline adsorption model parameters $\mathrm{pK}_{\mathrm{S}}^{\mathrm{int}}=6.1 \mathrm{pK}_{\mathrm{SC}}^{\mathrm{int^{* }}}=12.2 \mathrm{~m}_{\mathrm{i}} \omega=6.0 \mathrm{n}$ $=2.0$ were used to generate both tigures. 

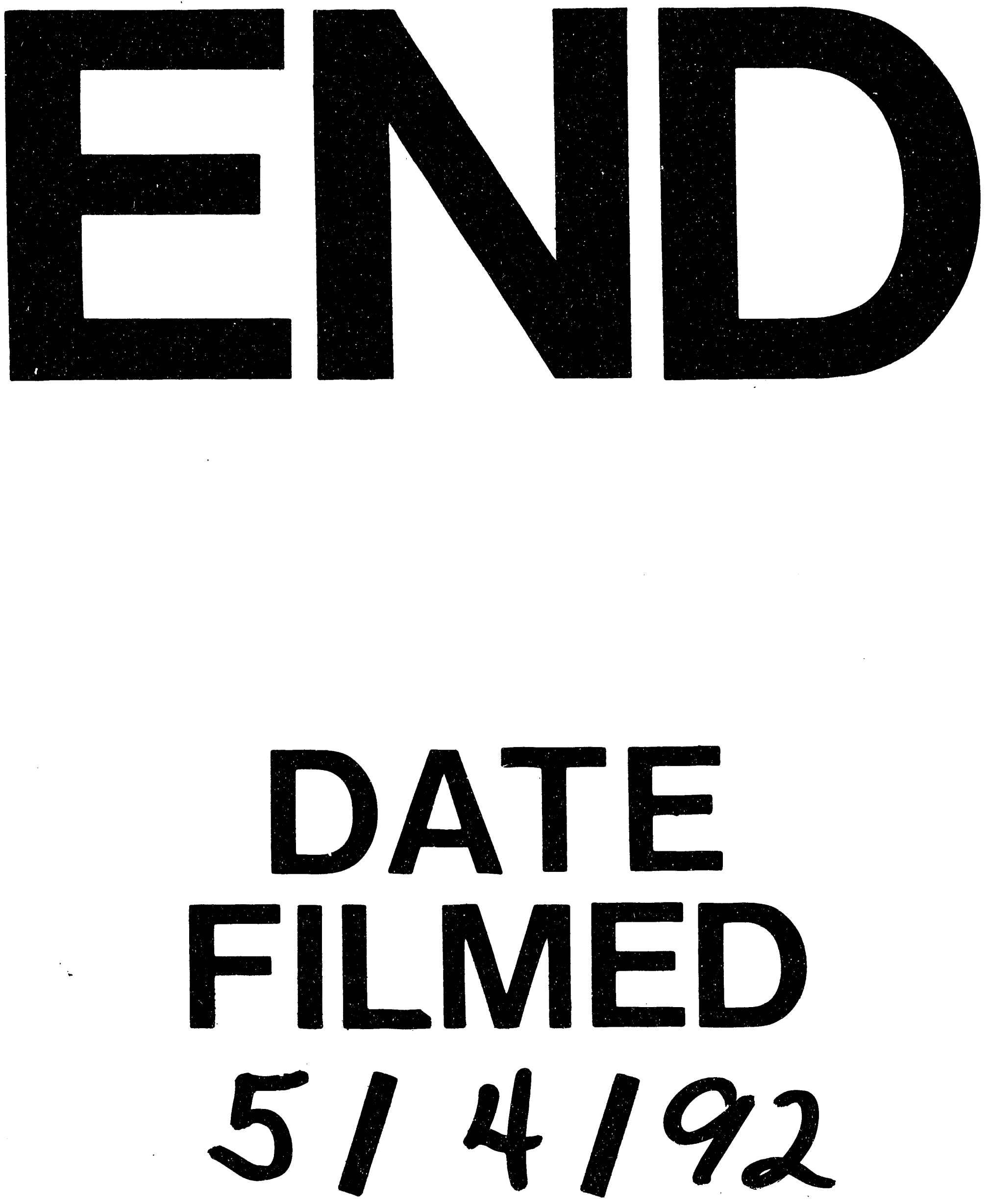
